\title{
A Framework for Environmental Risk Management
}

\section{Stafford Edward Charles Lloyd}

Thesis submitted in partial fulfilment of the degree of Doctor of Engineering in Environmental Technology

\author{
University of Surrey
}

Centre for Environmental Strategy

June 2013

Academic supervisors: Dr. Jacquetta Lee, Dr. Lucia Elghali and Prof. Chris France

Industrial supervisor: Dr. Andrew Clifton

Sponsoring organisation: Rolls-Royce plc 
ProQuest Number: 27606669

All rights reserved

INFORMATION TO ALL USERS

The quality of this reproduction is dependent upon the quality of the copy submitted.

In the unlikely event that the author did not send a complete manuscript and there are missing pages, these will be noted. Also, if material had to be removed, a note will indicate the deletion.

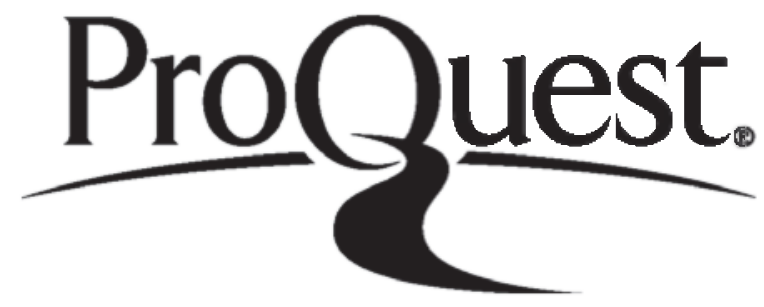

ProQuest 27606669

Published by ProQuest LLC (2019). Copyright of the Dissertation is held by the Author.

All rights reserved.

This work is protected against unauthorized copying under Title 17, United States Code Microform Edition (C) ProQuest LLC.

ProQuest LLC.

789 East Eisenhower Parkway

P.O. Box 1346

Ann Arbor, Ml 48106 - 1346 
'philosophers have only interpreted the world in various ways, ... the point is to change it'

Karl Marx, quoted by Adams (1995) and Robson (1993) 


\section{Acknowledgements}

This book is more than a thesis about a framework. It represents the culmination of a journey of personal discovery, not only of knowledge and how it is created, but of who I am. It also represents a physical journey. This research has taken me to some special places of which I have fond memories: Boston, Delft, Kyoto, Osaka, Yale University, The Brecon Beacons, The Houses of Parliament, to name a few.

Many people have helped me along this journey, almost universally with positive encouragement and good banter, who I would like to acknowledge:

My supervisors: Andy Clifton, Jaqi Lee, Lucia Elghali and Chris France. Andy, for all the good times we've had on our various travels, Jaqi, for being an important driving force throughout, Lucia, for sharing your knowledge and wisdom, and Chris, for giving me the final objective I really needed. I'd also like to say a special thank you to Nigel Bond at Rolls-Royce, who has been an unofficial supervisor all the way through.

My family: $\mathrm{Pa}, \mathrm{Ma}$, Tor, James and Harrison. Thanks Pa for a room and a desk, which provided the solitude I needed to finally get it finished, and $\mathrm{Ma}$, Tor, James and Harrison, for providing respite from writing. I'd also like to thank Nana, whose desire to see her grandson graduate provided real motivation.

l'd like to thank everyone at Bristol Sonics Rugby League club, and all the amazing friends l've made in Bristol over the past few years. The break provided by playing and coaching Rugby really did help keep me sane at times, and it's not an overstatement to say that I don't think I would have gotten through without it.

I'd also like to thank everyone l've met on the Surrey EngD, especially those who went on the Advanced Leadership course in 2010 , which I feel was a real turning point on my journey. I feel privileged to have been a part of the programme.

Finally, l'd like to thank Sally, for supporting me through the final stages and being there at the end. I'm looking forward to our next journey together... 


\section{Abstract}

This research has developed and tested a framework for environmental risk management. The framework provides a means for considering the environmental impacts of products as part of standard design decisions within Rolls-Royce by considering their relationship with other design requirements.

This research began during the development of an environmental assessment methodology within Rolls-Royce, which was to form the basis of a Design for Environment (DfE) capability. It had been successfully shown how the methodology could produce product life cycle environmental information in response to design inputs. However, it was not clear how this information should be used within design decisions. This EngD project was started to investigate how environmental impacts could be considered as part of standard design decisions within Rolls-Royce and to develop a bespoke decision support framework that could fulfil this requirement.

Aiming to produce outcomes that could lead to change within Rolls-Royce, an action research approach was applied. Initial research concluded that an approach to DfE consistent with existing ways of working needed to be developed and a risk based approach was selected as risk management is an important part of design at RollsRoyce. The framework for environmental risk management was developed to assess business risks posed by the environmental impacts of products alongside other risks as part of standard design and risk management processes.

To test the framework, focus groups were conducted to identify priority environmental business hazards that needed to be considered as risks in design. Case study scenarios based on these hazards were used to show how the risks posed to design objectives could be assessed, using actual design and business information within Rolls-Royce. Findings from testing the framework for environmental risk management culminated in recommendations on how to implement it into the business. A further contribution to knowledge was made in the form of a framework for conducting material criticality assessments, which was developed through testing the framework for environmental risk management. 


\section{Executive Summary}

\section{Introduction}

This research began in an engineering design department within Rolls-Royce during the development a Design for Environment (DfE) capability. Rolls-Royce makes power systems, predominantly based on gas turbine technology, providing solutions for customers in four markets: civil aerospace, defence aerospace, marine and energy (Rolls-Royce, 2013). This research has focused on the design of Rolls-Royce's aerospace products, as it was hosted within the aerospace parts of the business.

Setting the environmental context for the development of a DfE capability, the greatest environmental impacts over the life cycle of an aero engine are incurred during product use (Lee, 2006), to the extent that use phase and non-use phase environmental impacts are typically considered separately. The main environmental impacts from the use phase of aero engines include climate change, local air pollution around airports and noise (RCEP, 2002). Important environmental impacts from the non-use phases of the life cycle include the depletion of abiotic resources, energy use and greenhouse gas emissions, use of hazardous substances and waste (Delay-Saunders, 2012; RollsRoyce, 2013).

Rolls-Royce has an environmental strategy to address these environmental impacts, which includes addressing use phase environmental impacts during product design. Specific fuel consumption (a common measure of engine efficiency), weight, $\mathrm{NO}_{\mathrm{x}}$ emissions (related to air pollution) and noise are managed in design through well understood and defined requirements, which has enabled the company to make significant improvements in all of these areas (Beasley and Clifton, 2008). However, historically, attempts to address non-use phase environmental impacts in design have been less successful. A simplified environmental assessment tool was developed, although this is no longer used as the information provided by the tool was not relevant to standard design decisions. A process for reducing non-use phase environmental impacts in design has also been developed, however the appropriate environmental analysis tools were not available to fulfil the requirements of the process. Several barriers for implementing consideration for non-use phase environmental impacts into design decisions also became apparent, largely due to the complexity of the products Rolls-Royce makes.

Despite these barriers, an example where environmental impacts from product manufacture led to a significant impact on design objectives highlighted the need to ensure non-use phase environmental impacts are considered in design. To address 
this problem the business began to develop the DfE capability. Development progressed to the point of trialling a method for assessing the environmental impacts of products, which could produce results from the input of basic design information. However, the business identified the need to better understand how the environmental information supplied by this tool should influence engineering design decisions. This EngD project was started to address this problem by developing a bespoke decision support framework that would allow for the environmental impacts of products to be related to standard design requirements and included within design trade-offs.

Integrating environmental considerations into design decisions

An action research approach was applied, as the research was taking place within the social context of an organisation, with the aim of producing outcomes that could lead to change within that organisation. As is typical of action research, a cyclical research pattern was used, developing research themes, undertaking action within the problem situation to produce findings, and using the findings to develop new research themes. The first iteration of the action research cycle investigated how environmental considerations could be integrated into design decisions in Rolls-Royce through a review of academic literature on the topic of DfE. The following conclusions from this review were used to guide research direction:

- Developing a thorough understanding of the business and design context is an integral part of developing any approach to integrating environmental considerations into design decisions;

- A bespoke approach relevant to this context is required;

- Setting environmental priorities, in terms of what environmental impacts are addressed, and how these are measured and assessed with the use of methods and tools, is also a fundamental part of developing any approach to DfE;

- DfE requires a methodology to support the use of methods and tools;

- A study that sought to integrate environmental considerations into design at Pratt \& Whitney, another manufacturer of gas turbine aero engines, concluded that any approach must be consistent with existing design processes and apply metrics that can be used in design trade-offs.

Building on these conclusions, investigations focused on developing a greater understanding of the business and design context for this research. An interpretation of the organisational culture and structure within Rolls-Royce highlighted the importance of developing an approach for integrating environmental considerations into 
design decisions that was consistent with current processes and ways of working. A review of the Rolls-Royce design context also highlighted how this research needed to focus on detailed design decisions at a tactical design level, largely because at a strategic level environmental issues already have a strong influence on design decisions. Risk management was also highlighted as being an important part of design within Rolls-Royce.

Findings from informal interviews, with various environmental specialists, design managers and experts from within and outside Rolls-Royce, were used to conclude investigations into how environmental considerations could be integrated into design decisions at Rolls-Royce. The following points summarise the main findings from these interviews:

- To integrate environmental considerations into design decisions environmental information needed to be presented in a way that can be compared and traded with other design requirements;

- The approach needs to be integrative, working within existing processes, methods and tools;

- It was necessary to overcome what some environmental specialists called the 'so what' factor? In the past, environmental information has been provided to designers, although how this information is relevant to their design tasks has not been communicated clearly. This has led to designers finding the environmental information useful, although concluding 'so what?';

- There are perceptions that due to use phase environmental impacts dominating the life cycle environmental profile of the products sold by RollsRoyce, non-use phase environmental issues are insignificant and can be overlooked. Any approach needs to overcome this perception by highlighting the importance of addressing non-use phase issues in design;

- Clear environmental priorities are needed;

- Environmental information needed to quickly point to some form of tangible action to reduce environmental impacts.

From the review of the topic of DfE, investigations into the design and business context within Rolls-Royce and informal interviews, it was concluded that one way in which environmental considerations could be integrated into design decisions was through using risk. It was envisaged that the risk based approach would meet several important requirements for integrating environmental considerations into design decisions: 
- Would allow for environmental impacts to be related to standard design requirements and considered within design trade-offs;

- Highlight why environmental issues from the non-use phases of the product life cycle are important to address;

- Lead into activities to develop actions to reduce risk and hence also reduce environmental impacts.

\section{Development of the framework}

A new research theme was started to investigate how risk could be used to integrate environmental considerations into design decisions, leading to the development of the framework for environmental risk management. An obvious place to start was to review how risk is managed within Rolls-Royce more generally. From this review it was concluded that, as risk management was already part of standard design practice, what was required was a means of ensuring risks posed by environmental impacts were managed like any other risk as part of existing design processes. It was also concluded that risk was a very big topic and it was necessary to define more precisely the types of risks that this research sought to manage.

A review of academic literature on risk was used to investigate the topic, focusing on how risk was understood within the contexts of business, engineering and the environment. The review highlighted how there are many different perspectives on risk, although each perspective is defined by what is considered to be the source of risks (usually called hazards or events) and the receptor of interest in terms of assessing the consequences of risks. It was also discussed how risk terminologies are used interchangeably between different perspectives, creating confusion over the precise meaning of phrases used to describe the different activities undertaken to understand risk. This highlighted the importance of defining precisely the terminology to be used for this research.

Focusing on how this research sought to manage risks posed by environmental issues during the design of products, the sources and receptors model was used to define a perspective on risk for this research that identified a business as the receptor of risks that arise from the environmental impacts of business activities, which are effectively defined as the sources of hazards. This perspective on risk is the same as the one defined by Matten (1995), who outlined the different components of environmental risk. Matten also highlighted how environmental risks do not come from the environment itself but from stakeholder responses to actual, potential or perceived environmental 
impacts, which can make environmental risks particularly complex and difficult to manage.

In an attempt to achieve clarity and consistency on the terminology used for this research, the following terms were defined to describe this perspective on risk:

- Environmental business hazard: stakeholder responses to environmental impacts with the potential to cause harm to business objectives;

- Environmental risk: multiplying the probability of an environmental business hazard by its potential impact on business objectives;

- Environmental risk management: the activities of identifying, assessing and treating environmental risks.

Building on the defined perspective on risk for this research, the framework for environmental risk management (Figure 0.1) was developed to manage environmental risks like any other risk within design. The framework was developed based on the need to capture and visually represent the different activities required to manage environmental risks as part of design, and their interrelationships, including:

- The need to visually represent environmental business hazards and the design process;

- Representations of the activities of identifying environmental business hazards relevant to Rolls-Royce and prioritising those hazards that need to be managed as part of standard design activities;

- The need to consider how environmental business hazards may affect products in-service as well as new designs;

- A means of linking environmental business hazards and products together through the provision of some form of product-based Life Cycle Environmental Data (labelled as LCED within the framework). An environmental business hazard must be related to the environmental impacts of a product and so in order to identify what hazards are relevant to what products requires data on the environmental impacts of products;

- The standard Rolls-Royce risk management steps of assess and treat risks, which will need to be undertaken once it has been identified that a product is affected by an environmental business hazard. Some treatment actions may take place within design, which requires a link back to the design process. 


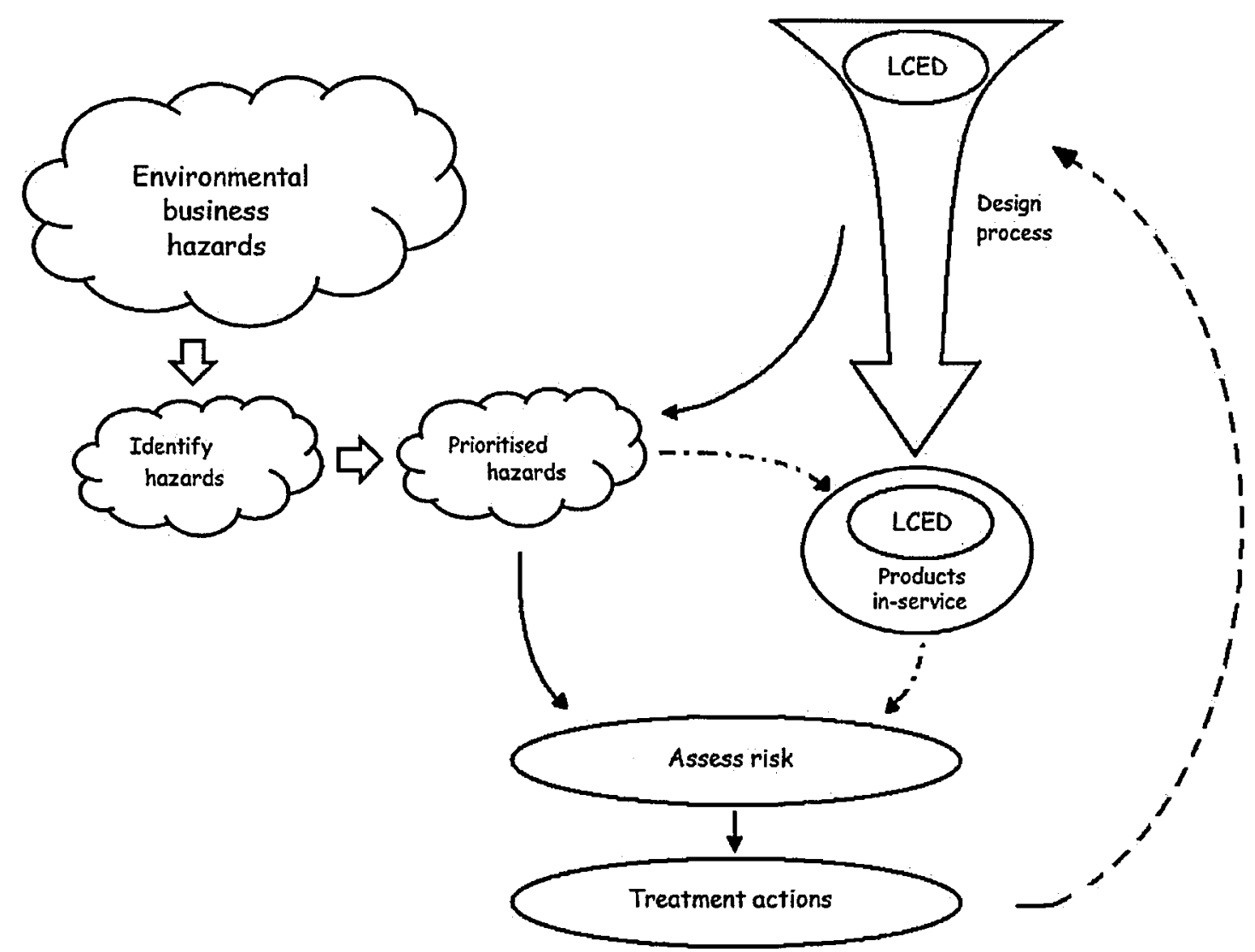

Figure 0.1: Framework for environmental risk management (adapted from Lloyd et al., 2012a)

\section{Environmental business hazards to be considered in design}

The next research theme focused on testing the framework, which began with research to identify environmental business hazards, presented in chapter 4 . The purpose of identifying hazards was not to produce a definitive list of hazards that were claimed to be the most important to Rolls-Royce, but to identify hazards that have been shown to be of concern to the business. These hazards could then be used to test the remainder of the framework. Findings from identifying hazards were also used to make recommendations on how to implement the framework into the business in chapter 7 .

Focus groups were selected as the research approach for identifying hazards, based on the need to capture judgements from environmental specialists and managers within Rolls-Royce on environmental business hazards and their relative importance. By allowing for group discussion and interactions it was judged that focus groups would produce better results than other research methods considered. From an analysis of focus group data the following hazards were identified:

- REACH (Registration, Evaluation, Authorisation and restriction of Chemicals): A European chemical regulation that seeks to ban or restrict the 
use of chemicals judged to be Substances of Very High Concern (SVHCs). Concerns over REACH dominated certain phases of the focus group discussions.

- Access to resources: Captured concerns over the scarcity of some materials used in Rolls-Royce products and how this posed a risk to the business, in particular how scarcity could lead to price increases and potential disruptions in supply. Concerns were also expressed about the potential use of materials sourced from conflict regions and how using these materials could impact on business reputation.

- $\mathrm{CO}_{2}$ /climate change and energy costs: Significant proportions of the focus group discussions focused on how climate change from carbon dioxide emitted over all phases of the life cycle of Rolls-Royce products could pose a risk to the business. Energy costs were selected as a hazard as some group participants voiced concerns over how increases in energy prices from regulation to address climate change would lead to unacceptable increases in product costs.

Using these hazards case studies were defined to test the remainder of the framework.

\section{Case study scenarios}

The first two case studies, presented in chapter 5, focused on assessing the risks posed by the access to resources hazard. A report from the European Commission (see European Commission, 2010) highlighted Rare Earth Elements (REEs) and Platinum Group Metals (PGMs) as having a particularly high supply risk. By identifying Rolls-Royce product designs that used these materials an assessment of the environmental risk was undertaken.

The first case study was based on the use of ruthenium, a particularly scarce PGM, in the intermediate pressure turbine blades of one of Rolls-Royce's newest large civil aerospace turbofan engines. A risk assessment was completed showing how potential increases in the price of ruthenium could have a significant impact on design unit cost objectives. One action to treat the risk would have been to remove the use of ruthenium from the engine, although this would impact on engine performance. An assessment of the risk posed to performance objectives from removing ruthenium showed how the risk posed to performance was less significant that the risk posed to cost and on balance ruthenium should be removed from the engine. The second case study was based on the use of yttrium, a rare earth element, in the coating on a combustion chamber on a small defence aerospace turbofan engine. An assessment 
of risks posed to design unit cost objectives showed that the impact was minimal, largely due to the very small amounts of yttrium used in the coating.

The main conclusions from the access to resources case studies included:

- Showed how the risk based approach would allow for environmental considerations to be related to standard design requirements and included within design trade-offs, which was one of the main requirements for the framework for environmental risk management.

- Highlighted how concerns related to access to resources were more focused on material accessibility rather than physical scarcity, and that the concept of 'materials criticality' was more closely aligned with how these risks needed to be captured.

- The method for assessing materials at risk, taken from the European Commission report, was also shown to be unsuited to Rolls-Royce's needs. Ideally the business needed to develop its own approach for assessing materials that are at risk, which reflected its own concerns. The business is now undertaking research to complete this activity at the recommendation of the researcher. A framework capturing the main steps required to complete criticality assessments was also developed (Figure 0.2 ), outlining how the business could identify critical materials using its own methodology.

- Confirmed the need for life cycle environmental data within the framework by demonstrating the need for information on where 'at risk' materials are used in products.

- Confirmed business concerns related to the access to resources hazard. In particular, concerns expressed by purchasing managers who recognised the benefits of influencing engineering material choices to mitigate risks. 


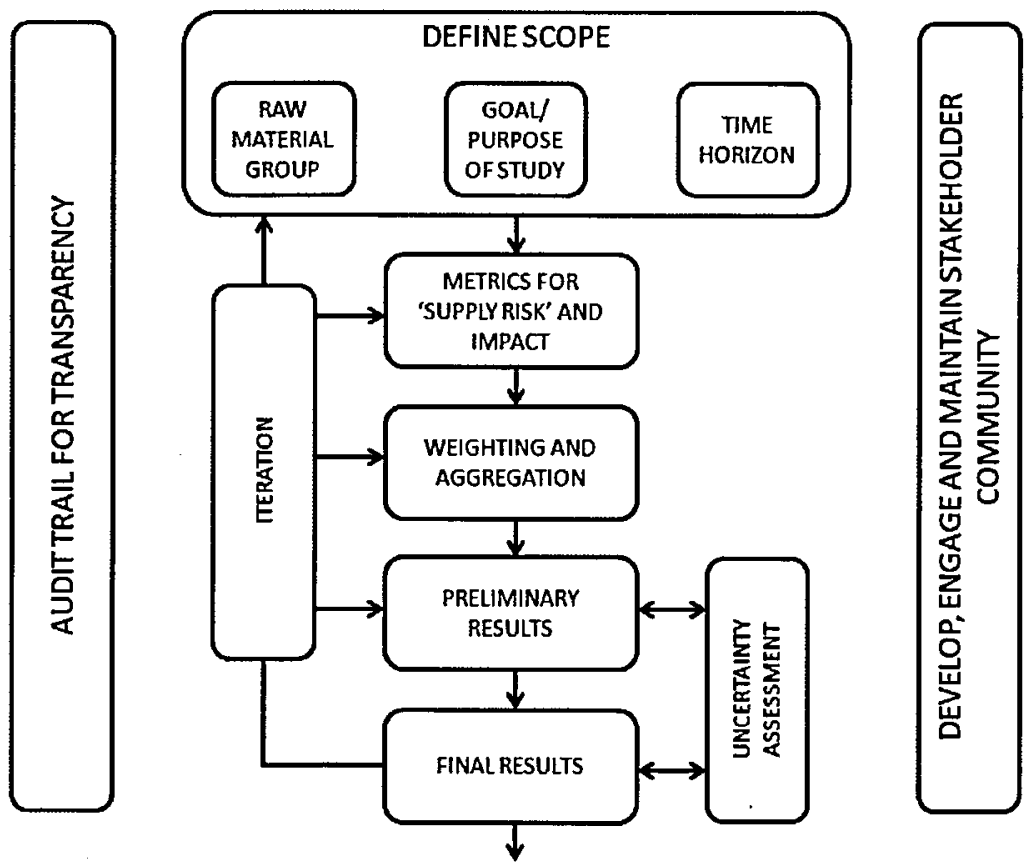

Figure 0.2: Framework for criticality assessments (Lloyd et al., 2012c)

Chapter 6 presented three further case studies focused on the REACH and energy use hazards. The first REACH case study assessed the risks posed by the use of zinc chromate, which has been classified as a SVHC under REACH due to its carcinogenic properties, on a small defence aerospace turbofan engine. Zinc chromate was used as a corrosion resistant coating primer on the fuel tank of the engine. The risk assessment result showed that the risk posed was significant, to the extent that it would require consideration from a higher management level within the business. The risk was large as if zinc chromate was banned by REACH effectively any engines that used it could not be manufactured and all business related to those engines would be at risk. At the time the case study was completed there were no known alternatives to zinc chromate in this application, meaning the only possible action to treat the risk would be to redesign the fuel tank with an alternative base material that would not require the same level of corrosion resistance. This would be a significant engineering task.

The second case study focusing on REACH assessed the risks posed by the use of trichloroethylene as a degreaser in the manufacturing route for a compressor stage of an advanced defence turbofan engine. Trichloroethylene is another known carcinogen classified as an SVHC under REACH. The result again showed that the risks were significant, as all business related to the engine was at risk if the substance became unavailable. However, in the trichloroethylene case risk treatment was much easier as the substance was used during manufacture as opposed to within the component, and a known alternative was available. This reduced what was a large risk to a very small 
one, as changing a process chemical would only require a minor engineering modifications, for example changing the specification on a drawing.

The final case study in chapter 6 focused on the risks posed by the energy hazard. Estimates of the amount of energy used to manufacture the same compressor stage that was the subject of the previous case study were used to assess how rises in energy costs could impact on engineering unit cost objectives. Estimates of the potential increase in the price of energy were calculated based on the price of carbon. Results showed that factoring in even the highest price of carbon projected in scenarios up to 2030 would not lead to a significant impact on engineering cost objectives from resultant increases in energy prices.

Conclusions from chapter 6 included:

- Results of the risk assessments reflected concerns expressed in the focus groups regarding the magnitude of the business risks posed by $\mathrm{REACH}$. Further studies are required to confirm the finding that risks posed by the energy hazard would not be significant in the foreseeable future.

- Risks posed by the REACH hazard are always going to be significant, as if a substance becomes unavailable, products cannot be manufactured and all business related to those products is at risk. However, there will be large differences in the residual risks posed from treatment actions, related to where the substance is used and whether alternatives are available. It was concluded that it would be sensible for the business to focus on the actions required to treat the risks posed by $\mathrm{REACH}$ to understand the potential impact on the business, rather than the initial risk posed by substances becoming unavailable.

- Further highlighted the need for life cycle environmental data within the framework, required to understand where substances affected by REACH are used in products and in the manufacturing processes and supply chains used to make them.

The main result from the case studies was that it had been successfully shown how the framework could be used to integrate environmental considerations into standard design decisions within Rolls-Royce by using risk.

\section{Recommendations for implementing the framework}

Having shown that the framework for environmental risk management has sufficient merit to be taken forward and implemented in Rolls-Royce, recommendations for 
implementation were taken from the findings from testing the framework, which included:

- The need for the business to develop and agree criteria to prioritise materials and substances, related to the access to resources and REACH hazards, which pose a risk to the business. These risks would then need to be managed by identifying where they are used in products and designs.

- Development of tools for automatically producing life cycle environmental data for designs, so it can be easily identified where products are affected by these hazards. The business has completed a parallel programme of research to develop a capability to produce this data, embedded within CAD systems.

- The need to consider how the management of environmental risks could be embedded within existing design and business processes, or creating new business processes where required.

- Agreeing methods for completing risk assessments, including assessing the probability and impact of environmental risks, consistent with existing risk management processes within Rolls-Royce.

- Considering the non-technical factors required to successfully implement the framework, including the need for senior management commitment.

- Identifying new environmental business hazards. A conceptual model for identifying hazards (presented in chapter 7) was developed through findings from the focus groups. It would also be necessary to consider how the management of new risks could be embedded within the organisation.

\section{Contributions to knowledge}

This research has made two contributions to knowledge. The first is the development of a risk based approach to DfE through the framework for environmental risk management and the testing of this framework (published in Lloyd et al., 2012a). The framework presents a visual representation of the different elements required to manage environmental risks in design, and the interrelationships between these elements, allowing for this concept to be applied within Rolls-Royce and other businesses. The framework was tested using case studies based on business information and design data within Rolls-Royce (an adaptation of one of the case studies was published in Lloyd et al., 2012b). Showing how the framework could work in practice was crucial to developing recommendations on how it could be implemented. 
The second contribution is the framework for conducting material criticality assessments, which was developed from merging findings from a review of materials criticality studies with pre-defined attributes of effective decision support in complex contexts (published in Lloyd et al., 2012c ${ }^{1}$ ). First defined by National Research Council (2008), materials criticality seeks to understand the risks posed by potential restrictions in the accessibility of materials, by assessing the probability of a material supply restriction (often termed 'supply risk') and the potential impact if a restriction were to occur. Whilst there are many materials criticality studies available (a comprehensive overview is provided by Erdmann and Graedel, 2011), to the researcher's knowledge there has been no description of the general steps required to complete a criticality assessment. The criticality framework is useful for organisations that may wish to conduct their own assessments, instead of relying on studies produced from other sources.

\footnotetext{
${ }^{1}$ Won a Telford Premium award, acknowledging papers of exceptional quality published in the Proceedings of the Institution of Civil Engineers.
} 


\section{Contents}

Acknowledgements iii

Abstract iv

Executive Summary v v

Contents $\quad$ xvii

List of figures $\quad$ xxi

List of tables $\quad$ xxiv

Glossary of terms and acronyms $\quad$ xxv

1 Introduction 1

1.1 Introduction to Rolls-Royce 1

1.2 Environmental context 3

1.2.1 Use phase environmental impacts 3

1.2.2 Non-use phase environmental impacts 5

$\begin{array}{lll}1.3 & \text { Rolls-Royce's environmental strategy } & 6\end{array}$

1.3.1 Design for Environment (DfE) 7

1.3.2 Development of the DfE capability 9

$\begin{array}{lll}1.4 \text { Aim, objectives and thesis structure } & 11\end{array}$

$2 \quad$ Integrating environmental considerations into design decisions $\quad 14$

$\begin{array}{lll}2.1 & \text { Methodology } & 14\end{array}$

2.2 Design for Environment (DfE) 17

$\begin{array}{ll}\text { 2.2.1 What is DfE and what are its benefits? } & 18\end{array}$

$\begin{array}{ll}\text { 2.2.2 Developing an approach to DfE } & 19\end{array}$

2.2.3 DfE tools 23

2.2.4 Examples of DfE within industry 27

$\begin{array}{ll}2.2 .5 & \text { Summary and conclusions } \\ 24\end{array}$

2.3 Understanding the context 35

2.3.1 Organisational culture and structure 36

2.3.2 Overview of design processes 40

2.3.3 Summary of the context and conclusions 50

2.4 Informal interviews 51 
2.5 Summary and conclusions 52

$3 \quad$ Developing the framework $\quad 54$

3.1 Methodology 54

3.2 Risk management within Rolls-Royce 55

3.2.1 Risk management structure and process 56

3.2.2 Engineering and risk management 61

$\begin{array}{ll}3.2 .3 & \text { Informal interviews }\end{array}$

3.3 Defining risk for this research 62

3.3.1 Risk in a business context 63

$\begin{array}{ll}\text { 3.3.2 Risk in an engineering context } & 69\end{array}$

$\begin{array}{lll}\text { 3.3.3 Risk in an environmental context } & 74\end{array}$

$\begin{array}{lll}\text { 3.3.4 Perspectives on risk: Summary and conclusions } & 77\end{array}$

$\begin{array}{ll}\text { 3.3.5 The perspective on risk for this research } & 78\end{array}$

3.3.6 Defining risk: Summary and conclusions $\quad 80$

3.4 The framework for environmental risk management 81

$\begin{array}{lll}3.5 & \text { Summary and conclusions } & 85\end{array}$

$\begin{array}{lll}4 & \text { Identifying environmental business hazards } & 87\end{array}$

$\begin{array}{lll}4.1 & \text { Methodology } & 87\end{array}$

$\begin{array}{lll}4.1 .1 & \text { Interviews } & 89\end{array}$

$\begin{array}{lll}4.1 .2 & \text { Focus groups } & 91\end{array}$

4.1.3 Delphi method 93

4.1.4 Scenario planning 95

$\begin{array}{ll}\text { 4.1.5 Selection of research approach } & 97\end{array}$

4.2 Research design $\quad 99$

4.2.1 Number of groups and participant recruitment 99

$\begin{array}{ll}\text { 4.2.2 Planning and running the groups } & 102\end{array}$

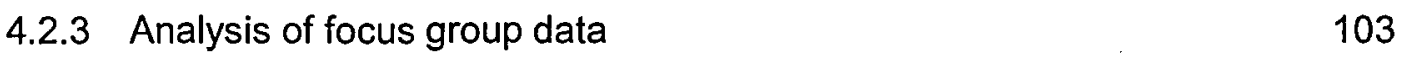

$\begin{array}{lll}4.2 .4 & \text { Role of the researcher } & 104\end{array}$

$\begin{array}{lll}4.3 & \text { Results } & 105\end{array}$

$\begin{array}{lll}\text { 4.3.1 Key-phrase analysis } & 105\end{array}$

$\begin{array}{ll}\text { 4.3.2 Output charts } & 107\end{array}$

$\begin{array}{ll}4.3 .3 & \text { Results summary } \\ & 120\end{array}$

4.4 Discussion 121

$\begin{array}{lll}4.4 .1 \text { Focus group results } & 121\end{array}$ 
4.4.2 The problem of identifying environmental business hazards 125

$\begin{array}{ll}\text { 4.4.3 Using focus groups to identify hazards } & 129\end{array}$

$\begin{array}{lll}4.5 & \text { Conclusions } & 130\end{array}$

$5 \quad$ Case study scenarios: Access to resources 132

$\begin{array}{lll}5.1 & \text { Methodology } & 132\end{array}$

$\begin{array}{lll}5.2 & \text { Access to resources } & 134\end{array}$

5.3 Case study scenarios 138

$\begin{array}{ll}\text { 5.3.1 Ruthenium in the Thames engine } & 139\end{array}$

$\begin{array}{ll}\text { 5.3.2 Yttrium in the Severn engine } & 148\end{array}$

5.4 Discussion 153

5.4.1 Results 153

$\begin{array}{ll}\text { 5.4.2 Risk assessment approach } & 154\end{array}$

$\begin{array}{ll}5.4 .3 & \text { Assessing probability } \\ & 155\end{array}$

$\begin{array}{ll}5.4 .4 \text { Assessing impact } & 156\end{array}$

5.4.5 Managing risks from the 'access to resources' hazard 157

$\begin{array}{lll}5.5 & \text { Summary and conclusions } & 158\end{array}$

6 Case study scenarios: REACH and energy 160

6.1 REACH and energy $\quad 160$

6.1.1 REACH hazard 160

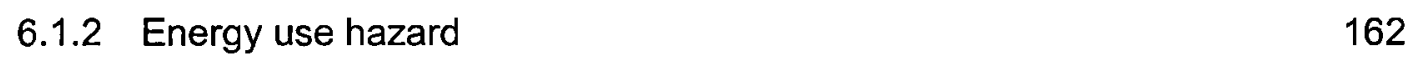

6.2 Case study scenarios 162

6.2.1 Zinc chromate in the Severn engine 163

6.2.2 Trichloroethylene on the Mersey engine 169

$\begin{array}{ll}\text { 6.2.3 Energy use and the Mersey engine } & 174\end{array}$

$\begin{array}{lll}6.3 & \text { Discussion } & 178\end{array}$

$\begin{array}{lll}\text { 6.3.1 Results } & 178\end{array}$

$\begin{array}{lll}\text { 6.3.2 } & \text { Risk assessment approach } & 180\end{array}$

$\begin{array}{lll}\text { 6.3.3 Assessing probability and impact } & 181\end{array}$

6.3.4 Managing risks posed by the REACH and energy hazards 182

$\begin{array}{lll}6.4 & \text { Summary and conclusions } & 183\end{array}$

$7 \quad$ Implementation of the framework $\quad 185$

7.1 Implementing the framework using identified hazards 186

$\begin{array}{lll}\text { 7.1.1 Hazard criteria } & 187\end{array}$ 
$\begin{array}{ll}\text { 7.1.2 Design workflow and product data } & 188\end{array}$

$\begin{array}{ll}\text { 7.1.3 Managing risks to in-service products } & 190\end{array}$

$\begin{array}{ll}\text { 7.1.4 Assessing probability and impact } & 191\end{array}$

$\begin{array}{lll}7.1 .5 & \text { Non-technical factors } & 192\end{array}$

7.2 Expanding the framework to manage new hazards 194

$\begin{array}{ll}\text { 7.2.1 Identifying more hazards } & 194\end{array}$

$\begin{array}{ll}\text { 7.2.2 Cross-functional working } & 196\end{array}$

$\begin{array}{ll}\text { 7.2.3 Practical requirements } & 197\end{array}$

$\begin{array}{lll}7.2 .4 & \text { Summary } & 198\end{array}$

$\begin{array}{ll}\text { 7.3 Applying the framework within other businesses } & 198\end{array}$

$\begin{array}{lll}7.4 & \text { Summary and conclusions } & 199\end{array}$

8 Conclusions $\quad 202$

$\begin{array}{lll}8.1 & \text { Research summary } & 202\end{array}$

8.2 Summary of findings and recommendations 205

8.3 Contributions to knowledge 208

8.4 Reflections on research findings and methods 209

8.5 Recommendations for further work 212

$\begin{array}{ll}\text { References } & 213\end{array}$

Appendix 1: Focus group key-phrase example 223

$\begin{array}{ll}\text { Appendix 2: Published papers } & \mathbf{2 3 0}\end{array}$ 


\section{List of figures}

Figure 0.1: Framework for environmental risk management (adapted from Lloyd et al., 2012a) $\mathrm{x}$

Figure 0.2: Framework for criticality assessments (Lloyd et al., 2012c) .................... xiii

Figure 1.1: The gas turbine aero engine (Phillips, 2008) ..................................... 2

Figure 1.2: Radiative forcing from aviation effects (Penner et al., 1999) ...................... 4

Figure 1.3: Sources of engine noise from modern aircraft and older designs (Phillips, 2008)

Figure 1.4: Simple linear product life cycle highlighting the non-use phases (adapted from Lee, 2006) 5

Figure 1.5: Rolls-Royce DfE materials selector (Payne, 2001) ............................... 8

Figure 1.6: Bypass duct with iso-grid feature (Beasley and Clifton, 2008) ................. 10

Figure 1.7: Representation of the environmental assessment methodology (Beasley and Clifton, 2008)

Figure 2.1: The in-context research framework with methods (adapted from Braa and Vidgen, 1999) 15

Figure 2.2: Cycle of action research (Checkland and Holwell, 1998, p.15)... 16

Figure 2.3: Action research cycle applied to the present problem (adapted from Checkland and Holwell, 1998) 17

Figure 2.4: Levels of ecodesign (Bhamra, 2004) ................................................... 20

Figure 2.5: Product system life cycle (Remmen et al., 2007) ................................. 21

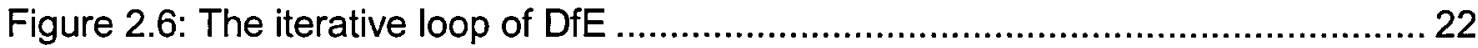

Figure 2.7: Phases and applications of LCA (Rebitzer et al., 2004) .......................... 24

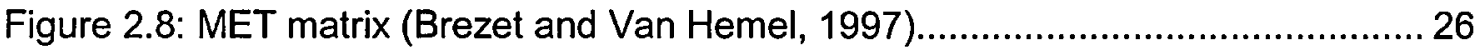

Figure 2.9: Environmental effects of design decisions at Airbus (Delay-Saunders, 2006)

Figure 2.10: Ford's Product Sustainability Indicators (Schmidt and Taylor, 2006)...... 30

Figure 2.11: Toyota Eco-VAS (Toyota, 2009) ...................................................... 31

Figure 2.12: Lucent Technologies PBEMS touch points (Donnelly et al., 2006).......... 32 Figure 2.13: Simplified Rolls-Royce business structure (for illustration only 4 SCUs are

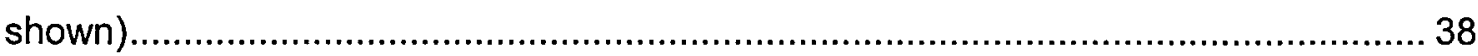

Figure 2.14: Strategic design context.......................................................... 41

Figure 2.15: Simplified Rolls-Royce engineering process (O'Toole, 2009)................. 43

Figure 2.16: Rolls-Royce PILM process (O'Toole, 2009) ...................................... 43

Figure 2.17: Design Review (DR) gates summary ............................................. 45

Figure 2.18: Risk management cycle (O'Toole, 2009) ........................................... 48 
Figure 3.1: Action research cycle for investigating using risk to integrate environmental considerations into design decisions (adapted from Checkland and Holwell, 1998).... 55

Figure 3.2: Risk management structure (adapted from Rolls-Royce, 2009) ............... 56

Figure 3.3: Risk management process (adapted from Rolls-Royce, 2009)................. 58

Figure 3.4: Example Probability Impact Diagram (PID) (Rolls-Royce, 2007b) ............ 59

Figure 3.5: Effects of risk treatment (Rolls-Royce, 2007b) ...................................60

Figure 3.6: Framework for managing risk (adapted from BSI, 2009) .........................64 64

Figure 3.7: Risk management process (adapted from BSI, 2009)............................66

Figure 3.8: Summary risk framework (RAEng, 2003a) ........................................ 74

Figure 3.9: Risk management cycle (Gerrard and Petts, 1998)..............................77

Figure 3.10: Components of environmental risk (adapted from Matten, 1995) ........... 79

Figure 3.11: Environmental business hazards and the design process ..................... 81

Figure 3.12: Identifying environmental business hazards.................................. 82

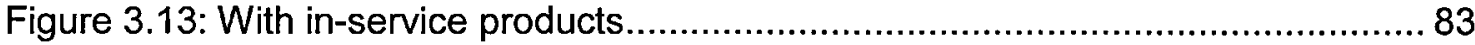

Figure 3.14: With Life Cycle Environmental Data (LCED) ..................................... 84

Figure 3.15: Framework for environmental risk management (developed from Lloyd et al., 2012a) 85

Figure 4.1: Action research cycle for identifying environmental business hazards (adapted from Checkland and Holwell, 1998) 87

Figure 4.2: Focus group output chart.................................................................... 104

Figure 4.3: Output chart from focus group one ............................................ 108

Figure 4.4: Output chart from focus group two .................................................. 114

Figure 5.1: Action research cycle for testing the framework (adapted from Checkland

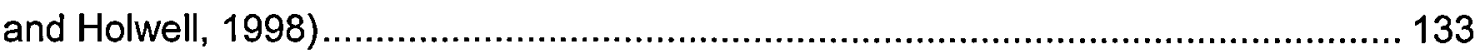

Figure 5.2: The concept of 'criticality' (National Research Council, 2008) ................ 136

Figure 5.3: Example Probability Impact Diagram (PID) (Rolls-Royce, 2007b)........... 136

Figure 5.4: Thames engine with location of IPT highlighted (Rolls-Royce, 2011) ...... 139

Figure 5.5: Thames engine unit cost PID ........................................................... 141

Figure 5.6: Use of ruthenium risk assessment result .......................................... 144

Figure 5.7: Risk assessment result using BGS probability assessment method........ 146

Figure 5.8: Risk assessment PID using sfc criteria ......................................... 147

Figure 5.9: sfc risk assessment result............................................................... 148

Figure 5.10: Low-bypass ratio turbofan combustion module (Rolls-Royce, 2007a) ... 149

Figure 5.11: 'Severn' engine combustion chamber cross-section ........................... 149

Figure 5.12: PID for Severn engine risk assessment .......................................... 150 
Figure 5.13: Risk assessment result for the use of yttrium on the Severn engine ..... 152

Figure 5.14: Framework for criticality assessments (Lloyd et al., 2012c).................. 156

Figure 6.1: PID for Severn engine risk assessment ......................................... 164

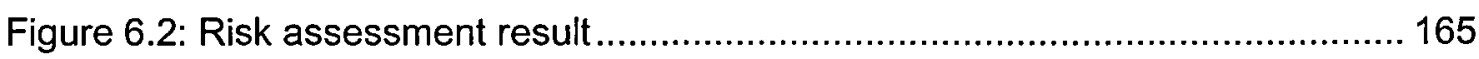

Figure 6.3: Risk management structure in Defence engineering............................. 166

Figure 6.4: Risk assessment result using engineering function risk criteria............... 167

Figure 6.5: Residual risk assessment result...................................................... 168

Figure 6.6: Mersey engine low-pressure compressor module (Rolls-Royce, 2007a). 169

Figure 6.7: Risk assessment PID (Rolls-Royce, 2009)......................................... 170

Figure 6.8: Risk assessment result .............................................................. 172

Figure 6.9: Risk assessment result with alternative probability ............................ 172

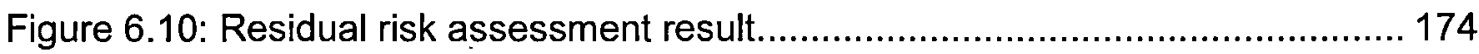

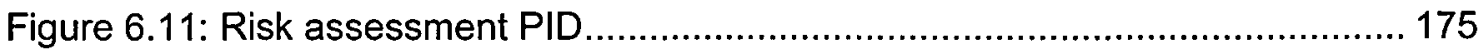

Figure 6.12: Historical energy price trends (DECC, 2009) .................................. 176

Figure 6.13: Risk assessment result ......................................................... 177

Figure 7.1: Summary of research completed to test the framework ......................... 185

Figure 7.2: Requirements for implementing the framework ................................... 187

Figure 7.3: Management of environmental risk in the design process..................... 189

Figure 7.4: Conceptual model for identifying environmental business hazards ......... 195

Figure 7.5: Cross-functional working required to implement the framework .............. 196

Figure 7.6: Hazard introduction process ............................................................ 197

Figure 8.1: Simplified action research model (adapted from Robson, 1993) ............ 211 


\section{List of tables}

Table 1.1: Summary of key non-use phase environmental issues (from DelaySaunders, 2006; Phillips, 2008; Rolls-Royce, 2013) ............................................6

Table 1.2: Summary of steps in the Rolls-Royce DfE process .................................. 9

Table 2.1: Common mid-point environmental impact categories (Wrisberg et al., 2002;

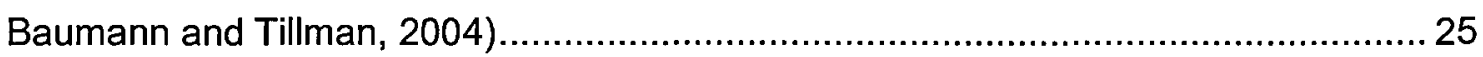

Table 2.2: Summary of generic DfE strategies (Brezet and Van Hemel, 1997) ........... 27

Table 2.3: Conceptual framework of organisational cultures (Handy, 1999; Harrison, 1972)

Table 2.4: Influencing factors and task types that could determine culture (Handy, 1999)

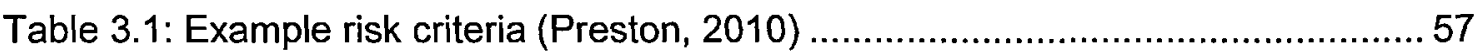

Table 3.2: Qualitative risk assessment criteria (Rolls-Royce, 2007b)....................... 59

Table 3.3: Summary of risk perspectives and terminology ................................... 78

Table 4.1: Summary of research methods reviewed ................................................ 98

Table 4.2: Job titles of focus group participants .................................................. 102

Table 4.3: Focus group key-phrases (listed alphabetically) .................................. 106

Table 4.4: Frequency of key phrases (results of both focus groups) ....................... 107

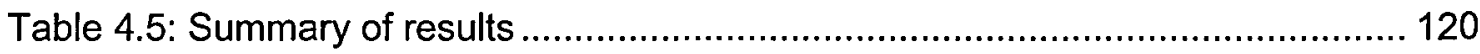

Table 5.1: Summary of supply risk metrics and underlying assumptions (Lloyd et al.,

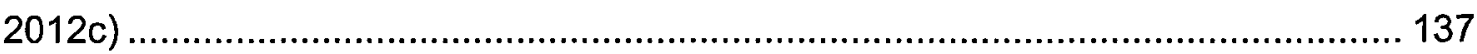

Table 5.2: Top five results for Supply Risk (SR) (European Commission, 2010)...... 138

Table 5.3: Thames engine risk criteria (Preston, 2010) ....................................... 140

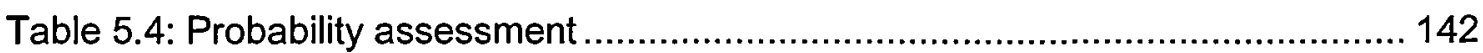

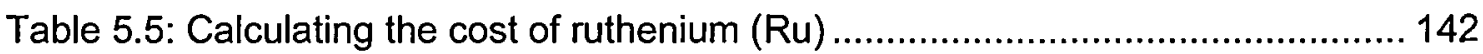

Table 5.6: Ruthenium impact calculations...................................................... 143

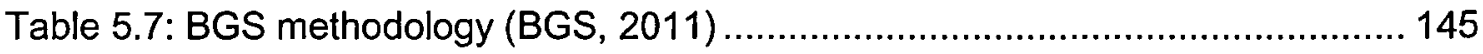

Table 5.8: Sample scores from the BGS method (BGS, 2011) ........................... 145

Table 5.9: Risk scoring scheme for BGS method, with PGM score highlighted......... 145

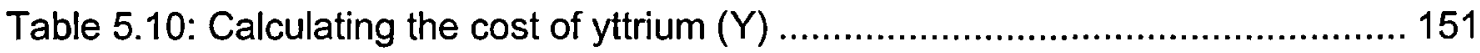

Table 5.11: Yttrium impact calculations ............................................................... 152

Table 6.1: REACH Probability scoring scheme.................................................. 165

Table 6.2: Engineering function risk criteria (Snape, 2009)................................. 166

Table 6.3: Electricity cost estimates........................................................... 177 


\section{Glossary of terms and acronyms}

\section{ACARE}

CDR

CFBU

Design process (within RollsRoyce)

Design requirement

DfE

DFMEA

ECHA

Environmental business hazard

Environmental risk

Environmental risk management

Gas turbine aero engine

LCA

LCED

$\mathrm{LCl}$

LCIA
Advisory Council for Aeronautics Research in Europe

Critical Design Review, a review gate in the RollsRoyce design process for approving a design before it is released to manufacture

Customer Facing Business Unit, a division of Rolls-Royce that is responsible for the running of programmes that supply completed products to customers

Series of linked steps describing how a product definition is derived from a set of requirements. Design decisions, which are referred to throughout this thesis, take place within this process

'Expression of a particular need by a customer or other stakeholder in a uniquely identifiable statement, which can be validated and against which a solution can be verified' (O'Toole, 2009)

Design for Environment, any design activity that focuses on the environmental impacts of a product. Synonyms include ecodesign, environmental design or green design

Design Failure Modes Effects Analysis, method applied at Rolls-Royce for managing technical risks within the design process

\section{European Chemicals Agency}

Stakeholder responses to environmental impacts with the potential to cause harm to business objectives

Multiplying the probability of an environmental business hazard by its potential impact on business objectives

Activities of identifying, assessing and treating environmental risks

A type of gas turbine engine developed specifically for powering aircraft

Life Cycle Assessment, a methodological framework for evaluating the environmental impacts of a product

Life Cycle Environmental Data, environmental data required within the framework for environmental risk management to link environmental business hazards with products

Life Cycle Inventory, step within LCA that collates an inventory of the inputs and outputs from a product system under study

Life Cycle Impact Assessment, evaluation of environmental impacts within LCA 


\section{LCM}

Life cycle

Materials criticality (or critical materials)

NPV

PDR

PID*

PILM

PLM

REACH

Risk assessment*

Risk identification*

Risk management*

Risk treatment*

SCU

Supply Risk (SR)

SVHC

Systems engineering

TYF

\section{Life Cycle Management}

'Consecutive and interlinked stages of a product system, from raw material extraction, or generation from natural resources, to final disposal' (BSI, 2006)

A concept that seeks to understand risks posed by potential restrictions in the accessibility of material resources

Net Present Value

Preliminary Design Review, a review gate in the Rolls-Royce design process that conducts the first detailed review of a design

Probability-Impact Diagram, used to assess the significance of risks during risk assessment within Rolls-Royce

Product Introduction and Lifecycle Management, a programme risk management process used within Rolls-Royce

Product Lifecycle Management, software used to store data on Rolls-Royce products, for example bills of materials

Registration, Evaluation, Authorisation and restriction of Chemicals

Assessing the likelihood and potential impact of a risk and calculating the product of the two to evaluate significance

Recording events that may impact on the objectives of an activity

Overall set of activities and processes used to manage risk

Actions to reduce risk to an acceptable level, which includes risk mitigation

Supply Chain Unit, a division of Rolls-Royce that is responsible for supplying engine modules that are assembled into a completed product by CFBUs

Used in the context of materials criticality to describe the probability that materials will be subject to supply disruptions

Substance of Very High Concern, as defined by REACH

An inter-disciplinary approach and means for realising successful systems

Ten Year Forecast

\footnotetext{
*Terms used within the context of risk in Rolls-Royce
} 


\section{Introduction}

This research began in an engineering design department within the Rolls-Royce Defence Aerospace business during the development of a Design for Environment (DfE) capability. The capability was being developed to ensure environmental impacts were appropriately considered within design decisions at Rolls-Royce, aiming to:

- Help Rolls-Royce meet its key environmental challenges;

- Respond to growing environmental pressures, related policies and regulations;

- Reduce business costs;

- Gain business advantages from addressing sustainability.

Development of the capability had progressed to the point of trialling a method for assessing the environmental impacts of products, which could produce results from the input of basic design information. However, it was not clear how the results provided by the environmental assessment method could be used within engineering design decisions, in particular because there was no obvious relationship between environmental impacts and standard design requirements (Beasley and Clifton, 2008). This Engineering Doctorate project was started to investigate how environmental considerations could be integrated into engineering design decisions within RollsRoyce and to develop a bespoke decision support framework for using environmental information in design, which could lead to the implementation of a DfE capability.

To introduce the research, this chapter presents an overview of Rolls-Royce and its business activities in section 1.1. Section 1.2 introduces the environmental context, providing an overview of environmental issues relevant to Rolls-Royce's products and operations. Section 1.3 covers activities undertaken within Rolls-Royce to reduce these impacts, including the events that led to the development of the DfE capability. Section 1.4 presents the research objectives and thesis structure.

\subsection{Introduction to Rolls-Royce}

Rolls-Royce makes power systems, providing solutions for customers in four markets: civil aerospace, defence aerospace, marine and energy. Rolls-Royce power system products and services are predominantly, although not exclusively, based on gas turbine engine technology, with more than 50,000 gas turbines in operation globally with over 600 airlines, 160 armed forces, 2,000 marine customers and energy customers in 120 countries. In 2012 group turnover was $£ 12,209 m$, producing a profit before tax of $£ 1,429 \mathrm{~m}$. Rolls-Royce is a global business, with significant manufacturing 
and operational facilities in the UK, Germany, Scandinavia, North America and Asia, and a network of offices in over 50 countries. The business currently employs over 40,000 people (Rolls-Royce, 2013).

Investment in technology is a key part of Rolls-Royce's business strategy, due to the high level of technical knowledge required to design, manufacture and maintain the products it sells and services. Figure 1.1 provides an overview of some of the technological complexities associated with a typical Rolls-Royce gas turbine product. Continued investment in technology is required to ensure the business can meet its customer requirements in the future. In 2012 the business invested $£ 919 \mathrm{~m}$ in research and technology. Two-thirds of this funding was directed towards improving the environmental performance of its products (Rolls-Royce, 2013).

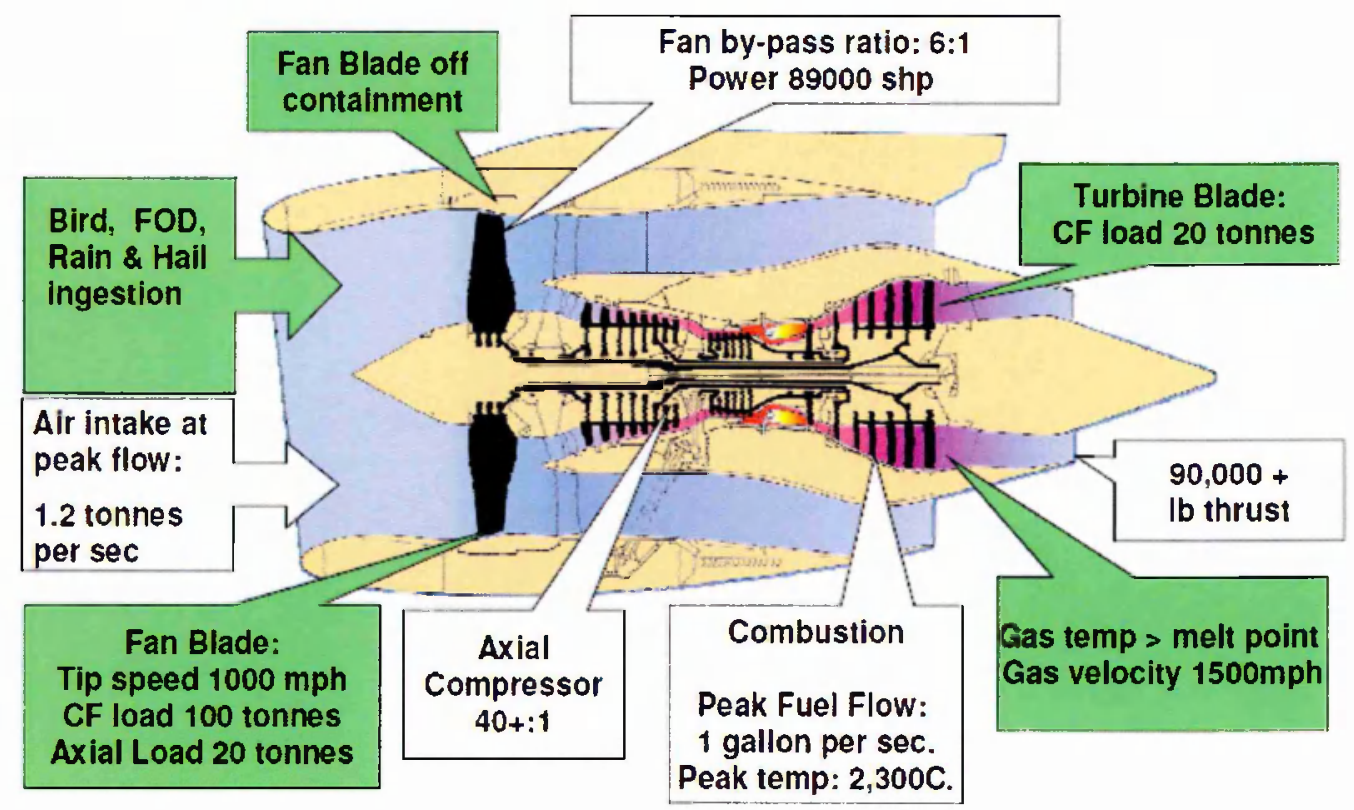

Figure 1.1: The gas turbine aero engine (Phillips, 2008)

Another important aspect of Rolls-Royce's strategy is its service-based business model. Rolls-Royce power systems can have a product life of several decades and a significant proportion of the revenue available to the business comes from maintaining products in-service. To maximise opportunities for services revenue, a significant proportion of Rolls-Royce products are sold through product service system agreements, which guarantee product availability for fixed price 'power by the hour' operational costs. In 2012 over $50 \%$ of Rolls-Royce's business revenue came from services (Rolls-Royce, 2013).

It is envisaged that a future DfE capability implemented as a result of this research could be deployed across all of Rolls-Royce's businesses. However, for the initial 
development of the capability, this research will focus on the design of Rolls-Royce's aerospace products, as it is being hosted within the aerospace parts of the business.

\subsection{Environmental context}

Focusing on aero engines, this section provides a summary of environmental issues over the life cycle of Rolls-Royce products. The greatest environmental impacts over the life cycle of an aero engine are incurred during product use (Lee, 2006), for example, over $99 \%$ of greenhouse gas emissions occur during the product use phase (Phillips, 2008). As there is such a big difference between use phase and non-use phase (i.e. material production, manufacturing, maintenance and disposal) environmental impacts, these are considered separately. Section 1.2.1 provides an overview of use phase environmental impacts. Non-use phase environmental impacts are covered in section 1.2.2.

\subsubsection{Use phase environmental impacts}

The main environmental impacts from the use phase of aero engines include climate change, local air pollution around airports and noise (RCEP, 2002). Contributions to climate change come from emissions of carbon dioxide $\left(\mathrm{CO}_{2}\right)$, water vapour $\left(\mathrm{H}_{2} \mathrm{O}\right)$, nitrogen oxides $\left(\mathrm{NO}_{\mathrm{x}}\right)$, sulphur oxides $\left(\mathrm{SO}_{\mathrm{x}}\right)$ and soot from the burning of kerosene during engine use (RCEP, 2002). It is easy to quantify how emissions of $\mathrm{CO}_{2}$ contribute to climate change by way of radiative forcing, as the gas mixes throughout the atmosphere and behaves like $\mathrm{CO}_{2}$ emitted from any other source (Penner et al., 1999). Radiative forcing effects from other gases are more difficult to quantify, especially as some emissions have globalised effects whereas the effects of others are limited to regions where aircraft fly. Being emitted at altitude, these emissions can alter the chemical composition of the atmosphere in a variety of ways (RCEP, 2002). Penner et al. (1999) detail the effects of these additional emissions, summarised in Figure 1.2.

The total effect on global warming from aviation has been estimated as 2.7 times that of $\mathrm{CO}_{2}$ alone, although this figure contains significant uncertainties (Penner et al., 1999). Emissions from civil aviation were estimated to account for approximately $2 \%$ of all anthropogenic $\mathrm{CO}_{2}$ emissions in 1992 , or about $3.5 \%$ of total radiative forcing from anthropogenic activities including the effects of other emissions (Penner et al., 1999). Both Penner et al. (1999) and RCEP (2002) overlooked emissions from defence 
aircraft within their studies, although an investigation by Rolls-Royce has shown these to be orders of magnitude lower than emissions from civil aviation (Lloyd, 2012a).

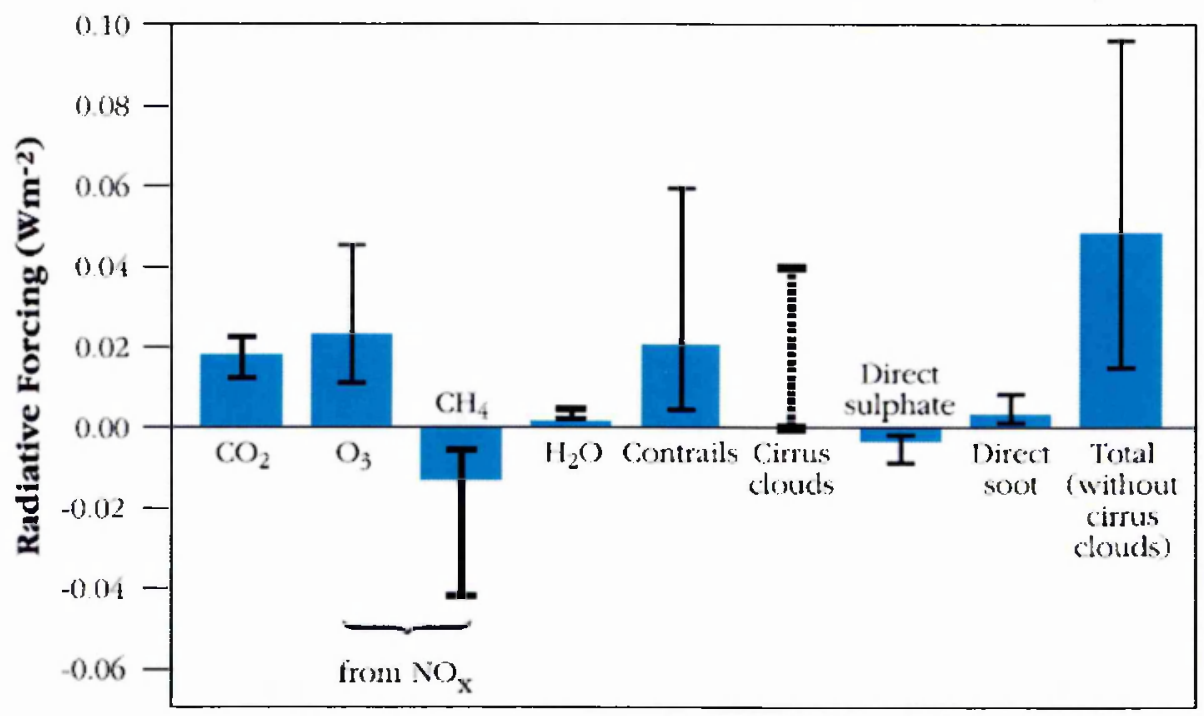

Figure 1.2: Radiative forcing from aviation effects (Penner et al., 1999)

Environmental impacts on local air pollution around airports come primarily from emissions of $\mathrm{NO}_{x}$ leading to photo-oxidant formation (Delay-Saunders, 2006; RCEP, 2002). $\mathrm{NO}_{x}$ is produced from a reaction between nitrogen and oxygen in the air during the combustion of kerosene at high temperatures within aero engines. Reducing impacts on local air pollution creates an environmental trade-off, as increasing engine temperatures to improve thermal efficiency is one way of making engines more efficient, although this increases $\mathrm{NO}_{\mathrm{x}}$ emissions (Penner et al., 1999). Meeting air quality standards presents the main challenge for reducing emissions of $\mathrm{NO}_{\mathrm{x}}$ as limits on levels of pollutants may restrict the expansion of some airports (RCEP, 2002).

Noise has been a long standing environmental issue within the aviation industry (Beasley and Clifton, 2008). Concerns focus on excessive noise around airports reducing the quality of life of local populations, leading to tangible psychological and physiological impacts to human health (Delay-Saunders, 2006). These impacts have led to specific legal restrictions on the permissible noise from aircraft, limited to decibel $(\mathrm{dB})$ levels appropriate to the local conditions of an airport. Significant efforts have been made to reduce the noise from aircraft in line with regulatory requirements and today's aircraft are typically $20 \mathrm{~dB}$ quieter than older designs, as illustrated in Figure 1.3 (Delay-Saunders, 2006). Noise presents another potential environmental trade-off, as the propulsive efficiency of aircraft can be improved by increasing bypass ratio, although an increased bypass ratio leads to a larger and noisier fan. 
Typical 1960s design

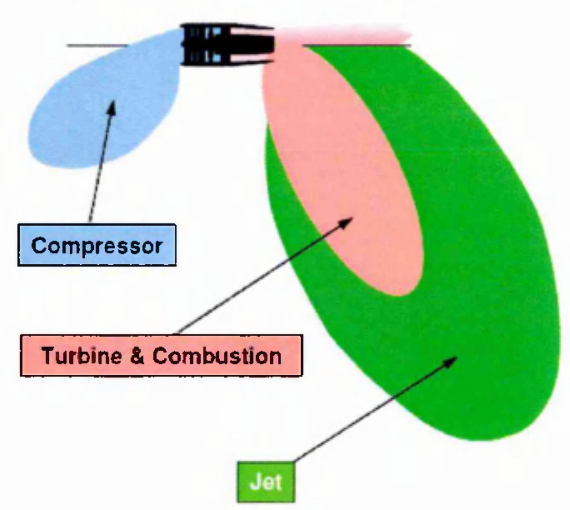

Typical modern design

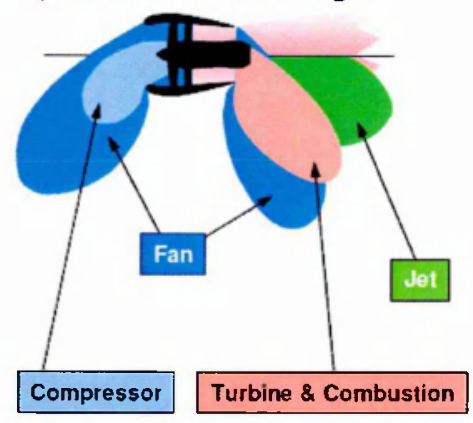

Figure 1.3: Sources of engine noise from modern aircraft and older designs (Phillips, 2008)

\subsubsection{Non-use phase environmental impacts}

There is a growing need to address environmental impacts from other phases of the aero engine product life cycle, highlighted in Figure 1.4, due to (from Delay-Saunders, 2006; Lee, 2006):

- Increasing amounts of environmental regulation;

- The need to respond to societal concerns surrounding the environmental impacts of the aviation industry;

- New Integrated Product Policy (IPP) measures, such as green public procurement and extended producer responsibility.

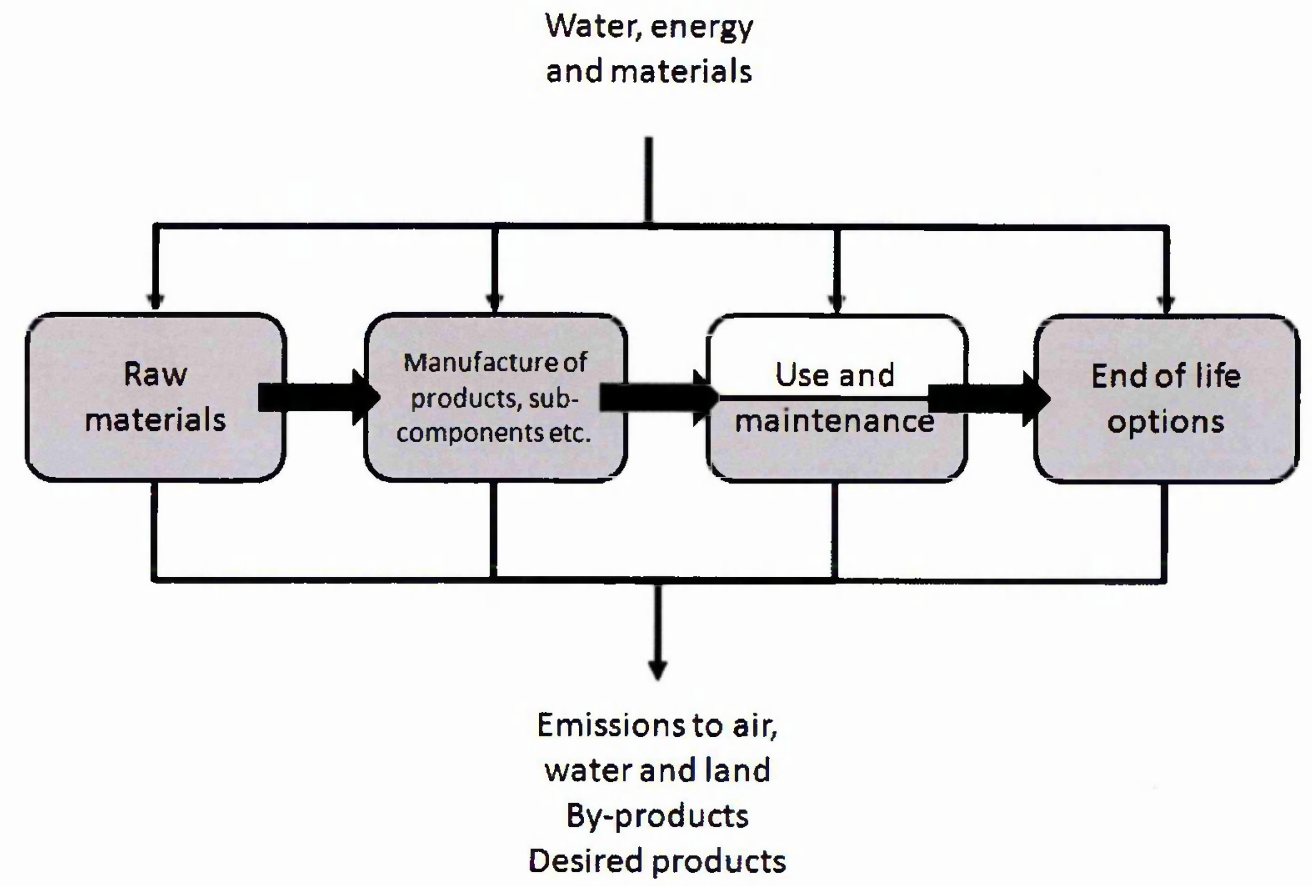

Figure 1.4: Simple linear product life cycle highlighting the non-use phases (adapted from Lee, 
Responding to these challenges, the Advisory Council for Aeronautics Research in Europe (ACARE) have set targets to achieve greener manufacturing, maintenance and disposal (Lee, 2006). Specifically, ACARE aims to minimise the use of resources, reduce harmful emissions to land, air and water and reduce the environmental hazards from materials and processes. Adopting a life cycle approach to reducing environmental impacts is highlighted as an important part of achieving these aims, as well as ensuring environmental risks are considered in design (Lee, 2006). Table 1.1 provides a summary of specific non-use phase environmental impacts, which can be targeted for achieving environmental improvements.

Table 1.1: Summary of key non-use phase environmental issues (from Delay-Saunders, 2006; Phillips, 2008; Rolls-Royce, 2013)

\begin{tabular}{|l|c|}
\hline \multicolumn{1}{|c|}{ Life cycle phase } & Key environmental issues \\
\hline Material production & $\begin{array}{c}\text { Depletion of abiotic resources } \\
\text { Energy, emissions and waste from material } \\
\text { production }\end{array}$ \\
\hline Manufacturing and maintenance & $\begin{array}{c}\text { Greenhouse gas emissions from energy use } \\
\text { Use of hazardous substances and materials } \\
\text { Hazardous processes } \\
\text { Waste generation, including solid waste } \\
\text { Resource efficiency } \\
\text { Water use }\end{array}$ \\
\hline End of life & Recycling and recovery of materials \\
\hline
\end{tabular}

\subsection{Rolls-Royce's environmental strategy}

This section summarises Rolls-Royce's environmental strategy, implemented to reduce the environmental impacts briefly covered in the previous section. From Rolls-Royce (2013), the business' environmental strategy focuses on three areas:

1. Supporting customers by further reducing the environmental impacts of products and services;

2. Developing new technology for future low emission products;

3. Maintaining a drive to reduce the environmental impacts of business activities.

A commitment to reducing environmental impacts is also demonstrated through RollsRoyce's membership of the Dow Jones Sustainability Index.

To reduce the environmental impacts of products and services Rolls-Royce works within environmental goals set by ACARE. In 2012 the business renewed its commitment to work towards the ACARE goals for 2050 (against a 2000 baseline), including (from Rolls-Royce, 2013): 
- Reducing aircraft $\mathrm{CO}_{2}$ emissions by $75 \%$ per passenger kilometre;

- Reducing noise by $65 \%$;

- Reducing $\mathrm{NO}_{\mathrm{x}}$ emissions by $90 \%$.

As mentioned previously, the business invests the majority of its research spending on developing technologies to improve the environmental performance of products and meet these targets. There is also a direct synergy between reducing the environmental impacts of products through reducing fuel consumption and meeting customer demands for more efficient aircraft to reduce operational costs. This helps to drive improvements along with industry targets such as those agreed by ACARE.

Activities to develop new technology for low emission products focus on expanding Rolls-Royce's nuclear capability within its energy business and working with partners to ensure future alternative fuels will be viable for aviation. Rolls-Royce has supplied and supported nuclear power plants for the UK Royal Navy's submarine fleet for many decades and intends to use this knowledge and expertise to expand its business into the civil nuclear market. Activities to approve alternative fuels for future use in aviation are governed by Rolls-Royce's fuels policy, which states that any new fuel must have a sustainable supply, not compete with food crops and must also be used in the most efficient way to minimise greenhouse gas emissions (Rolls-Royce, 2013).

Efforts to reduce the environmental impacts of business activities focus on reducing greenhouse gas emissions and maintaining environmental management certification of all relevant sites to ISO 14001 standards (see BSI, 2004). In 2012 the business had reduced carbon emissions by $16 \%$ (normalised by turnover, from a 2009 baseline). Rolls-Royce continues to be a leading member of the carbon disclosure project and absolute greenhouse gas emissions from operations were $550 \mathrm{ktCO}_{2} \mathrm{e}$ in 2012 (RollsRoyce, 2013).

\subsubsection{Design for Environment (DfE)}

The environmental impacts of products are an important consideration in design decisions at Rolls-Royce, which helps the business fulfil its environmental strategy. Environmental impacts from the use phase of aero engines are the most important design consideration after product safety, in particular for civil aerospace engines, although these are becoming increasingly important for defence products. Specific fuel consumption (a common measure of engine efficiency), weight, $\mathrm{NO}_{\mathrm{x}}$ emissions and noise are managed in design through well understood and defined requirements, which 
has enabled the company to make significant improvements in all of these areas (Beasley and Clifton, 2008).

Efforts have also been made to integrate considerations for non-use phase environmental impacts into design, to help the business meet commitments to reduce the environmental impacts of its operations and work towards the ACARE goal of greening manufacturing, maintenance and disposal through the adoption of life cycle approaches. Measures to consider non-use phase impacts have included the use of materials and process selectors and the development of a design standard for DfE.

Figure 1.5 provides an overview of the materials selector, which uses a traffic light hazard rating system to help identify hazardous materials in designs, or those that are less resource efficient or cannot be recycled. The process selector is based on a similar principle.

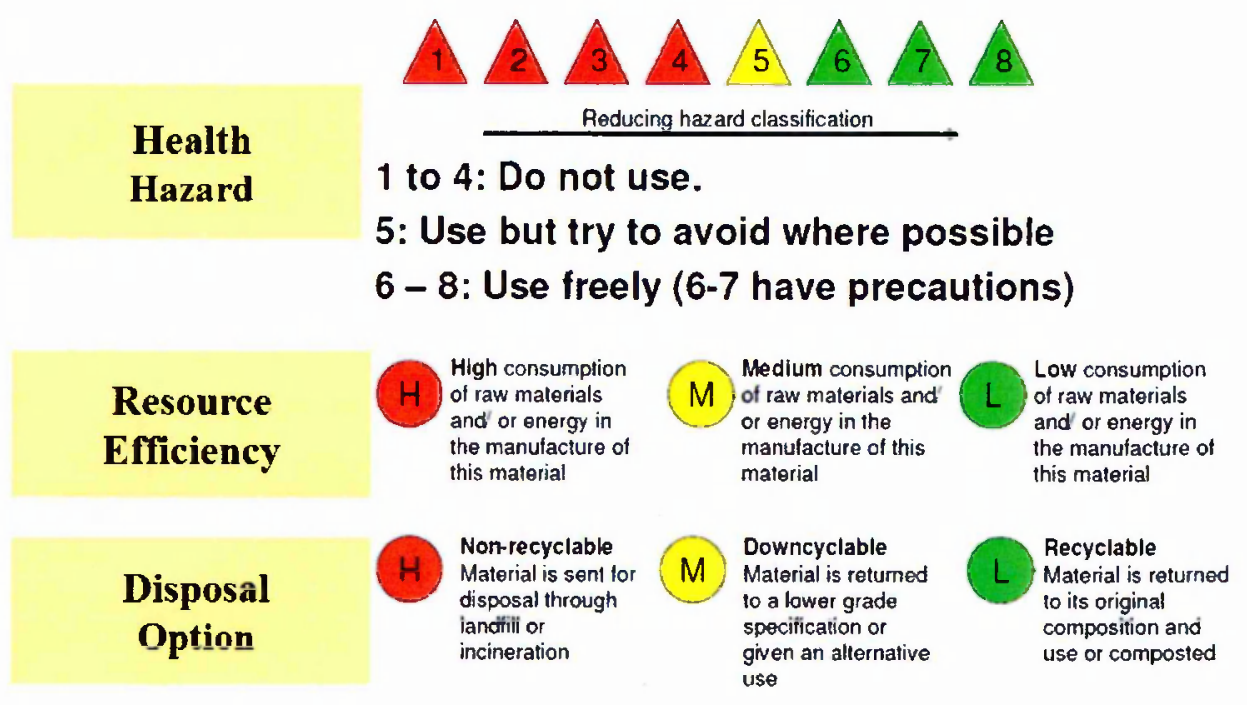

Figure 1.5: Rolls-Royce DfE materials selector (Payne, 2001)

In practice it has been found that the selectors were inadequate for integrating considerations for non-use phase environmental impacts into design decisions and they are no longer used. Even though materials and processes that have a high environmental impact are highlighted, the selectors do not provide designers with alternatives that could offer improvements. Sufficient information on how the materials and processes have a high environmental impact is also not provided, which does not make clear, in terms designers will understand, the consequences of choosing high impact materials and processes. It is also not clear how the information provided by the selectors could be used when making design trade-off decisions. These factors combined resulted in a situation where most designs carried an environmental statement based on information provided by the selectors, although to a large extent the information was overlooked. 
A process for DfE was developed to overcome the limitations of the material and process selectors. Table 1.2 summarises the main steps contained within the process.

Table 1.2: Summary of steps in the Rolls-Royce DfE process

\begin{tabular}{|l|l|}
\hline \multicolumn{1}{|c|}{ Engineering process step } & \multicolumn{1}{c|}{ Actions } \\
\hline Preliminary concept definition & $\begin{array}{l}\text { Identification of significant environmental aspects and } \\
\text { agreed project targets }\end{array}$ \\
\hline Full concept definition & Creation of top-level environmental impact statement \\
\hline Product realisation & $\begin{array}{l}\text { Development of quantified environmental impact } \\
\text { statements identifying areas of concern }\end{array}$ \\
\hline Production & $\begin{array}{l}\text { Measured environmental impact from key areas and } \\
\text { identification of unforeseen environmental risks }\end{array}$ \\
\hline Service support & $\begin{array}{l}\text { Risk management and environmental responsibility during } \\
\text { service }\end{array}$ \\
\hline Disposal & $\begin{array}{l}\text { Environmental impact of disposal in accordance with } \\
\text { agreed end of life plan }\end{array}$ \\
\hline
\end{tabular}

Whilst the new DfE process appeared to be appropriate in theory, in practice it could not be implemented, as there were no suitable environmental analysis tools developed to meet the requirements of the process. Several barriers to implementing the process also became evident during its development. Lee (2006) discusses how these barriers to DfE tend to be experienced across the aerospace industry, due to the complexity of the products manufactured, including (from Lee, 2006):

- Long-life products: as discussed aero engine designs can have a product life of several decades and it is difficult to foresee what environmental problems need to be addressed over this time horizon.

- Technical maturity: aerospace products have developed and improved incrementally over many years. This creates resistance to changing from established designs, amplified by the need to ensure product safety.

- Design freedom: technical maturity also leads to a lack of design freedom and a lack of alternatives to using designs that have significant non-use phase environmental impacts.

\subsubsection{Development of the DfE capability}

Despite the unsuccessful implementation of the DfE selectors, design process, and the barriers listed, there are examples that highlight the importance of considering non-use phase environmental impacts in design decisions at Rolls-Royce. One example highlights the importance of considering impacts from the manufacturing phase in particular, which was encountered when designing the bypass duct for one of RollsRoyce's latest defence turbofan engines. An important feature of the bypass duct 
design was an iso-grid visible on the outside of the component, which is shown in Figure 1.6. Reinforcing ribs created by the iso-grid help to provide structural integrity, whilst reducing the weight of the component. The original method for manufacturing this complex feature was chemical etching.

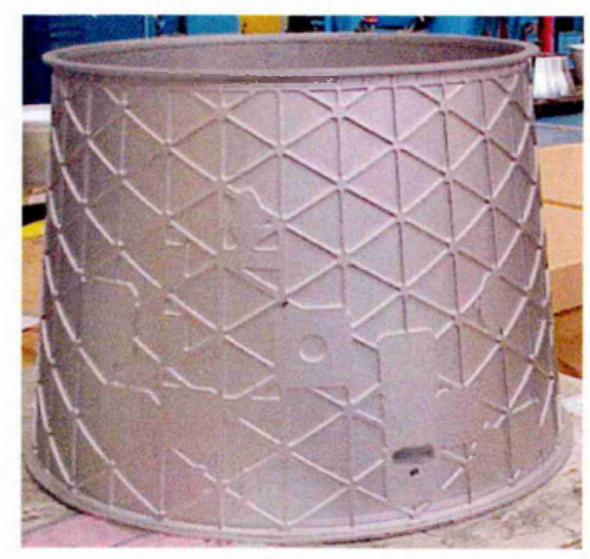

Figure 1.6: Bypass duct with iso-grid feature (Beasley and Clifton, 2008)

During detailed design work it became clear that the actual cost of the component was far higher than anticipated. It was discovered that the root cause of this cost increase was the cost of disposing of the environmentally harmful acid waste produced from the chemical etching process. A cost reduction exercise was launched and fortunately a suitable alternative manufacturing method was found to reduce the costs of the component, although this was only implemented after a lengthy development programme.

This example shows how environmental impacts from the non-use phase parts of the life cycle can have a significant impact on design objectives. It was anticipated that the occurrence of similar problems would become more likely in the future, due to increasing environmental concerns and regulations. To manage these issues proactively, it was agreed to develop a design capability for assessing the life cycle environmental impacts of products. The aim of the capability was to allow for areas of environmental concern to be identified and related to standard design requirements (Beasley and Clifton, 2008).

The first part of the capability developed was an environmental assessment methodology, which was based on a simplified life cycle approach. The methodology applies process models that are specifically developed for the unique requirements of the life cycle of gas turbine engines. The process models provide data on the environmental aspects of materials, processes and operations used to manufacture gas turbines. A designer can construct a model of the product's environmental life cycle by selecting the process models to be used across the product's life for a 
particular design, forming a process chain. An important feature of the methodology is a link between process model outputs and design parameters, meaning environmental aspects can vary in response to design inputs. Figure 1.7 provides a representation of the environmental assessment methodology (Beasley and Clifton, 2008).

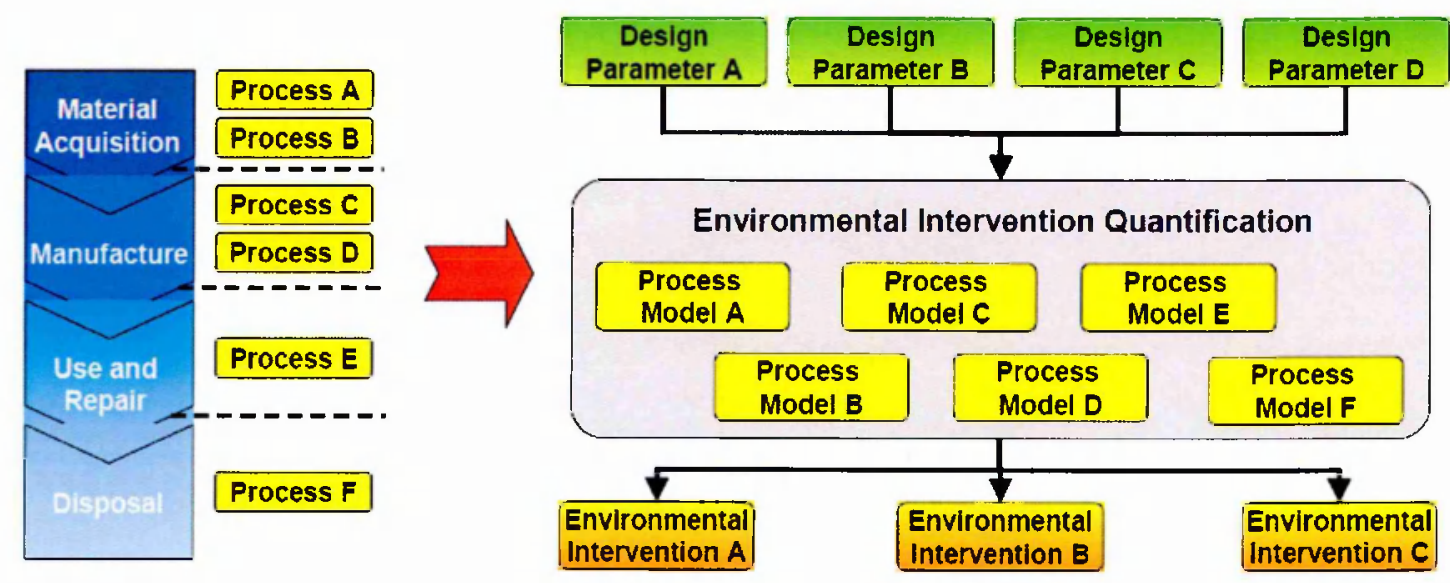

Figure 1.7: Representation of the environmental assessment methodology (Beasley and Clifton, 2008)

The methodology was trialled successfully, being used to create simplified life cycle environmental inventories for selected components, which highlighted areas of environmental concern. However, it was not clear how the information contained within these inventories could be translated into a format that could be used as part of standard design decisions.

\subsection{Aim, objectives and thesis structure}

Following on from the discussions in previous sections, the aim of this research was defined as:

- To develop a bespoke decision support framework that will allow for environmental considerations to be integrated into standard design decisions within Rolls-Royce, in a way that also allows environmental considerations to be traded-off with other design requirements, contributing to the future implementation of a DfE capability.

At the beginning of this research it was clear that a lot of time had been spent developing and trialling the environmental assessment methodology, and researching how this could be developed into a DfE tool. However, it was not clear how the environmental aspects collated for a design by this tool could be assessed as part of standard design decisions. Before a decision support framework could be developed, 
the first problem to be addressed was gaining a thorough understanding of design processes, and decisions made within these processes, leading to the first research objective:

1. To investigate how environmental considerations could be integrated into design decisions within Rolls-Royce, through understanding the design context and reviewing academic literature on the topic of DfE.

Findings from this investigation will point to a way of integrating environmental considerations into design decisions, which will need to be explored in order to develop the framework, leading to the second research objective:

2. To investigate the chosen approach for integrating environmental considerations into design decisions and develop the decision support framework. This will also require a review of the appropriate business processes and decision contexts within Rolls-Royce, and related academic literature.

Once the framework has been developed it will need to be tested, leading to the third research objective:

3. To test the framework through appropriate research activities, showing how it can be used to integrate environmental considerations into design decisions within Rolls-Royce.

The ultimate aim of this research is to produce outcomes that could lead to change within Rolls-Royce and the eventual implementation of a DfE capability, leading to the fourth research objective:

4. To use the findings from testing the framework to provide recommendations on implementation into the business.

This thesis is structured as follows:

Chapter 2 addresses the first research objective by reviewing the literature on DfE, the Rolls-Royce design context, and presents the findings from informal interviews focused on the topic of integrating environmental considerations into design decisions at RollsRoyce, to define an approach for achieving this.

Chapter 3 addresses the second research objective by describing the development of the bespoke decision support framework. The framework is developed by reviewing the appropriate academic literature and business contexts within Rolls-Royce. 
Chapter 4 begins to test the framework by undertaking research activities to establish the priority environmental business hazards that need to be considered within design decisions in Rolls-Royce.

Chapters 5 and 6 continue to test the framework by using these priority hazards as the basis for case studies, showing how they can be integrated into design decisions using Rolls-Royce design data.

Chapter 7 builds on discussions and findings from testing the framework in chapters 4,5 and 6 to discuss how it could be implemented into the business.

Chapter 8 present conclusions, including discussions on the research methods used and requirements for further work.

Research methods applied throughout this research are presented and justified at the beginning of the relevant chapters. 


\section{Integrating environmental considerations into design decisions}

This chapter presents the research activities undertaken to investigate how environmental considerations could be integrated into engineering design decisions at Rolls-Royce. Section 2.1 presents the methodology applied for this part of the research. Section 2.2 reviews the academic literature on the topic of Design for Environment (DfE) to gain insights into how an approach to integrating environmental considerations into design decisions could be developed at Rolls-Royce. Following on from this review, section 2.3 provides more detail on the context of Rolls-Royce's business and design processes. Section 2.4 summarises findings from informal interviews conducted with environmental specialists, design engineers and managers from within Rolls-Royce, and other businesses, which focused on the topic of integrating environmental considerations into design decisions. Section 2.5 concludes with an approach for how environmental considerations could be integrated into design decisions, which is developed in chapter 3.

\subsection{Methodology}

This section presents the research methods used to investigate how environmental considerations could be integrated into design decisions within Rolls-Royce. The selection of approach was based on the fact that the research was taking place incontext within an organisational setting, with the aim of producing outcomes that could lead to change within that organisation.

Researching in-context presented difficulties when it came to methodology, as solving real-world problems is not a recognised academic discipline (Checkland, 1993). As this research was socially situated, research approaches applied in the social sciences were deemed to be suitable (Braa and Vidgen, 1999). In particular, research methods geared towards tackling problems that seek to gain understanding and encourage change to promote the use of technical information within an organisation, namely integrating environmental considerations into design decisions.

Braa and Vidgen (1999) present a research framework for in-context organisational research, focused on the development and use of information systems. Research methods suitable for in-context research are identified as case studies, action research, field experiments and hybrids of the three (defining the action case approach). Superimposing research methods onto their framework, Braa and Vidgen (1999) align action research with change, case studies with understanding and field experiments with prediction (Figure 2.1). Given the aim of this research is to produce outcomes that may 
lead to changes within an organisation, action research is the most appropriate research approach.

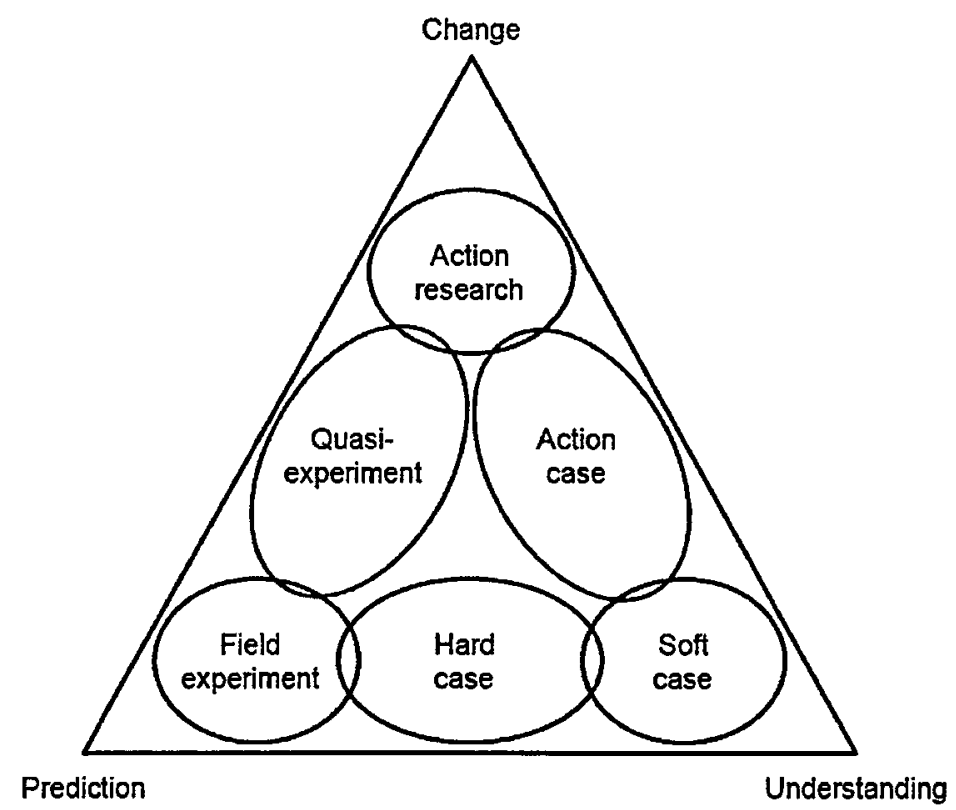

Figure 2.1: The in-context research framework with methods (adapted from Braa and Vidgen, 1999)

Action research is a social research method that has three distinctive characteristics (McNiff and Whitehead, 2000):

1. Researchers explicitly aim to work within the real world social context that they are studying;

2. The goal is to improve the situation that is being researched or produce outcomes that lead to change within this situation;

3. A cyclic research pattern is applied, acting, evaluating and reflecting on the results of action, in order to guide further research direction and produce findings.

A commonly cited origin of action research is Kurt Lewin's work on intergroup relations (Lewin, 1946). Lewin observed the need for action orientated research embedded within the social situations being studied, relying upon evaluation and reflection on the action as a means of achieving practical improvements. Action research is not a singular discipline and has evolved as a research approach within many different fields (Brydon-Miller et al., 2003). Some examples include the study of human relations, society, organisations, policy and psychology (Dash, 1999). As a result, action research comes in many different 'flavours' (Braa and Vidgen, 1999, p.30), ranging from less structured and more reflective methods such as community based action research (Stringer, 1999) to more structured approaches such as soft systems methodology (Checkland, 1993). 
Less structured approaches to action research are purely interpretivist. However, more structured approaches, which can still be labelled interpretivist (Rose, 1997), lean more towards positivism, striving to produce outcomes that could have a 'truth claim' (Checkland and Holwell, 1998, p.16). For the purposes of this research, the leaning towards positivism' perspective on action research will be adopted. This perspective is more suitable as it applies action research more as a method for structuring an enquiry into real-world problem situations, whereas less structured approaches apply action research as a means of studying social interactions. Applying action research as a method for structuring an enquiry is based on a view of the world as 'an exceptionally complex mess' (Checkland and Poulter, 2006, p.154), which the researcher shares.

As discussed, one of the main characteristics of action research is the cyclical and iterative research pattern, involving action, evaluation and reflection, leading to further action. There are many interpretations of this research cycle. Given the perspective of this research, the interpretation offered by Checkland and Holwell (1998) was applied (Figure 2.2).

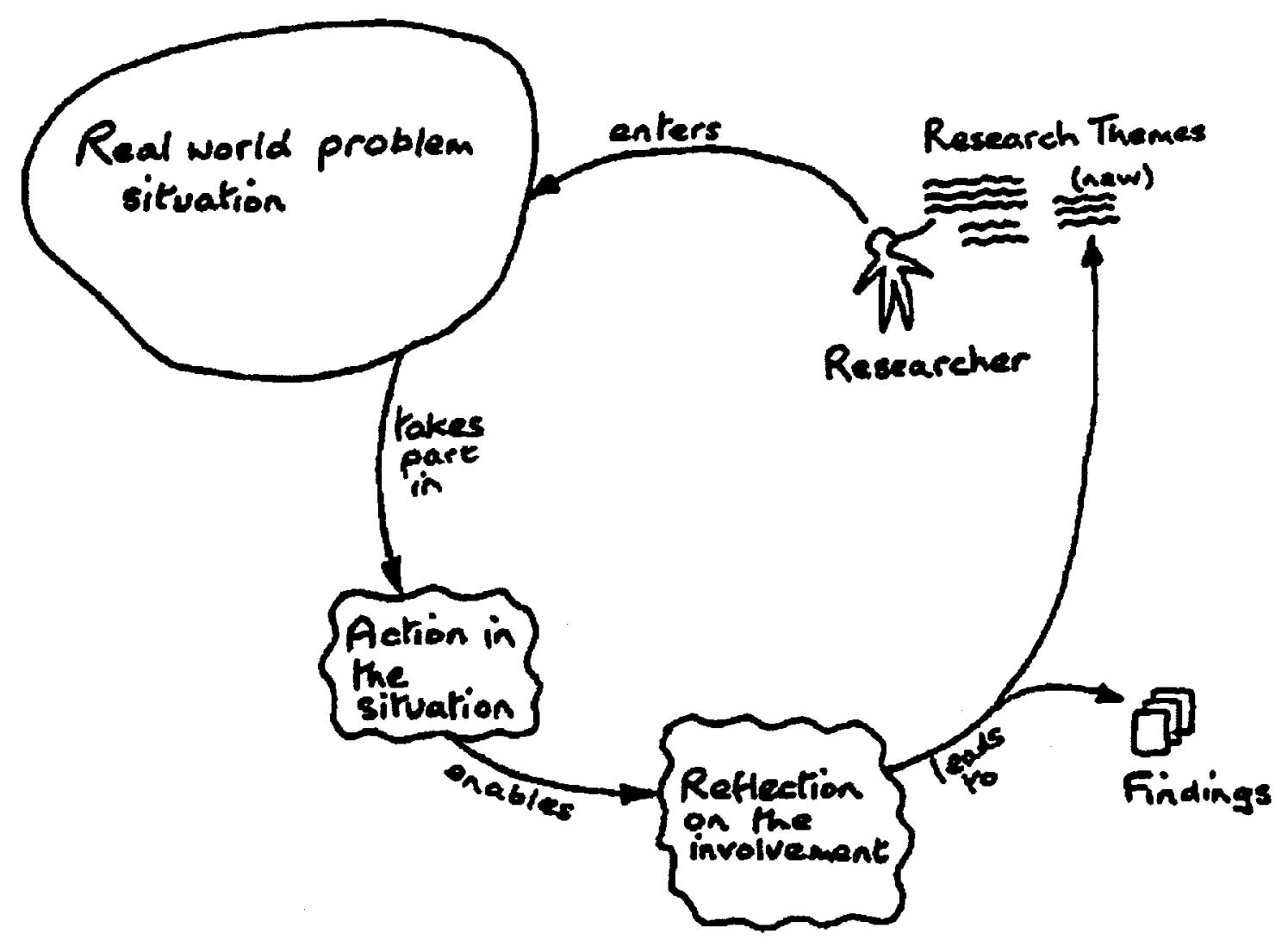

Figure 2.2: Cycle of action research (Checkland and Holwell, 1998, p.15)

From Figure 2.2, the research cycle begins with the definition of a research theme and the researcher entering a problem situation with the aim of investigating this theme. Action is then undertaken in the situation, leading to reflection, findings and new research themes, re-starting the iterative action research cycle. Applying the cycle for 
this research, the theme was defined as integrating environmental considerations into design decisions, with the researcher entering the problem situation of an engineering design department within Rolls-Royce. As the research was just beginning, action within the situation needed to be exploratory, understanding the problem situation in order to guide the development of new research themes, re-starting the cycle. Two common forms of exploratory research are literature reviews and interviews, the latter being particularly useful as a 'strategy for discovery' (Fielding and Thomas, 2001). Figure 2.3 adapts the action research cycle to the present situation. Findings from the first loop of the cycle lead to further iterations in later chapters.

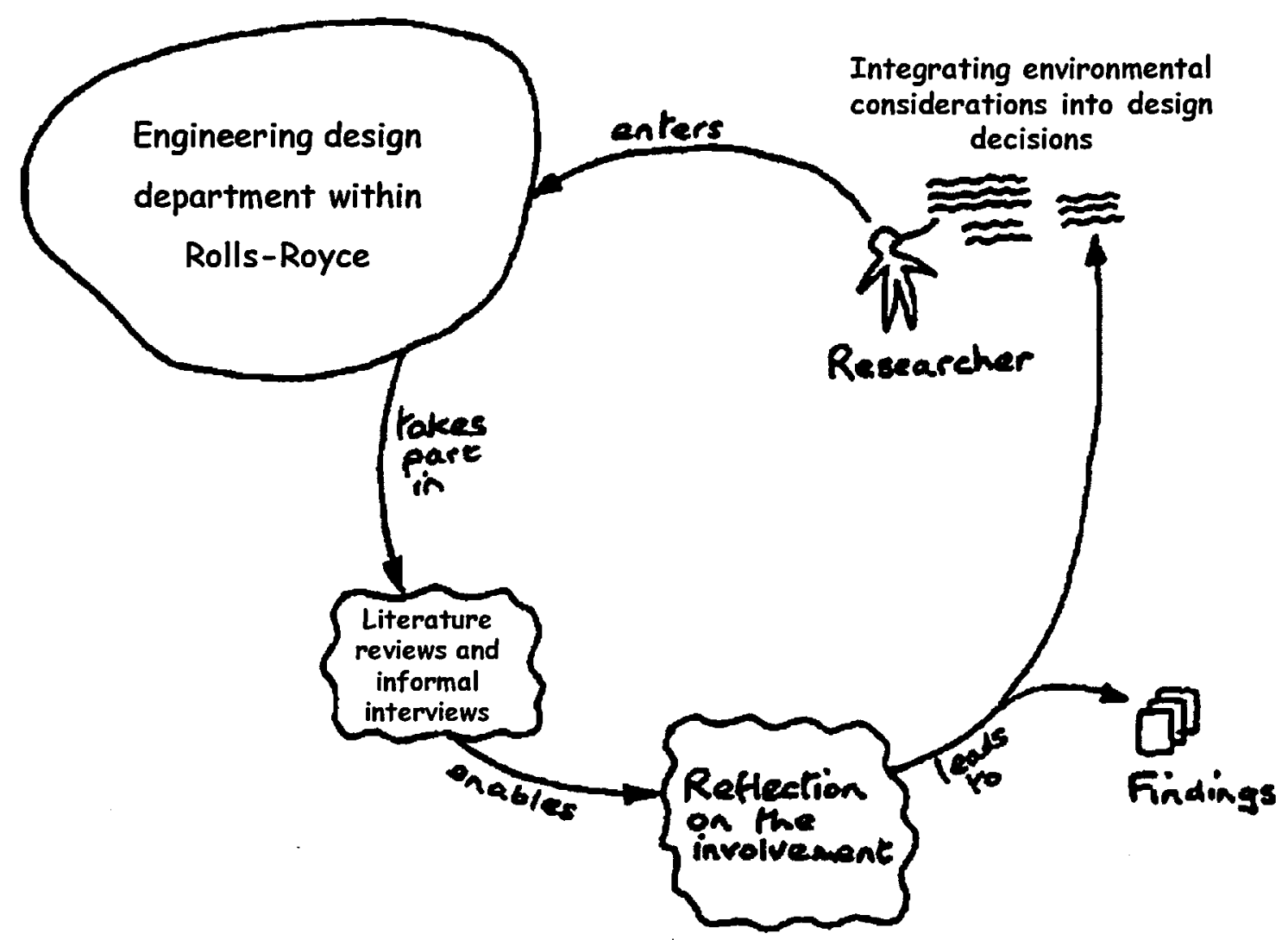

Figure 2.3: Action research cycle applied to the present problem (adapted from Checkland and Holwell, 1998)

The next section presents the first step of the research cycle, a review of academic literature on the topic of Design for Environment (DfE), to provide insights into how environmental considerations could be integrated into design decisions at Rolls-Royce.

\subsection{Design for Environment (DfE)}

This section reviews the academic literature on the topic of Design for Environment (DfE). Section 2.2.1 provides a general introduction to what DfE is and how businesses benefit from implementing it. Section 2.2.2 provides an overview of what 
needs to be considered in developing an approach to DfE, followed by a description of DfE tools in section 2.2.3. Section 2.2.4 provides examples of DFE being implemented within industry, leading into conclusions in section 2.2.5.

\subsubsection{What is DfE and what are its benefits?}

Taking design as the process of defining a physical product to fulfil human needs (Spangenberg et al., 2010), DfE can be defined as a 'systematic process by which firms design products in an environmentally conscious way' (Baumann et al., 2002, p.413), or more broadly, any design procedure that focuses on the environmental attributes of a product (Hauschild et al., 2004). The main challenge for DfE is to couple the product development processes used within businesses with the product life cycle (Veroutis and Fava, 1997), effectively marrying how businesses design products with considerations for their environmental impacts. The product life cycle is a basic underlying concept of DfE (Bakker, 1995; Fiksel, 1996), which can be defined as 'consecutive and inter-linked stages of a product system, from raw material extraction, or generation from natural resources, to final disposal' (BSI, 2006).

There are many synonyms for DfE, for example ecodesign, environmental design or green design (Bakker, 1995). More recently there has been a shift towards sustainable design, or Design for Sustainability (DfS), which encompasses social and economic concerns as well as environmental ones (Crul et al., 2009). For the purposes of this research, DfE is defined as distinct from DfS by only focusing on environmental problems.

Several generic drivers for the development of DfE activities are commonly referred to in the literature (from Bhamra, 2004; Brezet and Van Hemel, 1997; Handfield et al., 2001):

- The potential to reduce costs;

- Increasing amounts of environmental legislation and regulation;

- The effect good or bad environmental performance can have on a business' reputation;

- Demands from customers and market opportunities for 'green' products and services;

- Advantages over competitors provided by better environmental performance.

The most obvious reasons for implementing DfE are the pro-active and preventative approach to dealing with environmental problems it promotes (Bhamra, 2004) and its 
focus on products and their life cycle. DfE allows a preventative approach as 'once a product moves from the drawing board...its environmental attributes are largely fixed' (Baumann et al., 2002, p.413). It makes sense to address environmental problems before they get locked in (Charter and Belmane, 1999; Van Weenen, 1995) and it is stressed in the literature that to achieve maximum benefits, environmental considerations must be incorporated into design procedures as early as possible (BSI, 2002; Sroufe et al., 2000). A product focused approach is beneficial as it allows for the extension of environmental considerations across the whole product life cycle, allowing a more holistic approach than typical site based environmental management (Ammenberg and Sundin, 2005).

\subsubsection{Developing an approach to DfE}

This section briefly covers initial considerations in developing an approach to DfE, including:

- Systems perspectives;

- DfE and its links with life cycle thinking;

- Non-technical considerations, related to the role organisational complexities play in the success or failure of DfE (Boks, 2006).

\subsubsection{Systems perspectives}

Thinking at the systems level allows for more significant environmental improvements and should be the starting point for any approach to DfE (Hauschild et al., 2004). Systems level thinking starts by considering the functionality that a product provides and how best, from an environmental perspective, this functional requirement can be met by the supply of services and products. Levels of improvement can be identified as shown in Figure 2.4. 


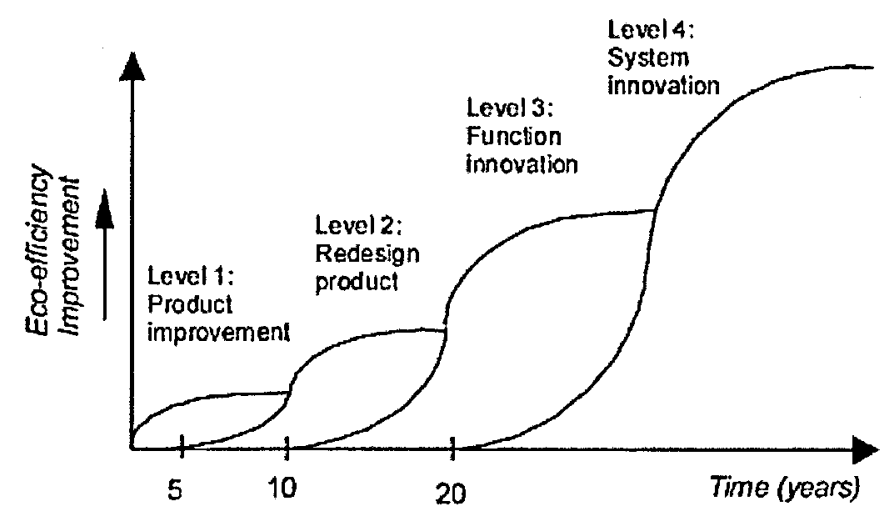

Figure 2.4: Levels of ecodesign (Bhamra, 2004)

In increasing complexity, product improvement is the lowest level strategy for environmental improvement, followed by product re-design, functional improvement and system level improvement. Using the example of the function provided by aero engines for air travel from $A$ to $B$ :

- A level 1 innovation would be a more efficient aero engine;

- Level 2 would be a step change environmental improvement in aero engine design, for example an engine which produces thrust using an open rotor;

- Level 3 would be selling power, the service provided by aero engines;

- Level 4 would call into consideration the need for air travel and thus aero engines. Would it be better, environmentally, to use another means of transport for getting from $A$ to $B$ ?

As demonstrated by this example, in order to achieve system level improvements (level 4), the sphere of influence tends to extend beyond the scope of a single organisation, requiring the inclusion of multiple stakeholders, changes in infrastructure and considerations for social attitudes. Due to the added complexity of a system innovation, environmental design is limited to the first 3 levels of improvement (Wrisberg et al., 2002).

Level 3 functional innovation improvements focus on shifting from selling physical products to the services they provide, as highlighted in the example above. Commonly described as Product Service Systems (PSS) or Sustainable Product Systems (SPS), service based business models offer greater potential for environmental improvements, as these decouple a business' profit from its material throughputs (Jackson, 1996). A further benefit of service based business models is that some responsibility for the product resides with the manufacturer, meaning that end of life considerations are more aligned with the business' interests (Maxwell and van der Vorst, 2003). 
More recently, there has been a desire to separate the development of PSSs into a separate field, reducing the dominance of the 'ecodesign community' to involve business and consumer sciences fields (Mont and Tukker, 2006, p.1453). Level 1 and 2 improvements are the most common focus for environmental designers. Level 2 product re-design strategies are usually labelled as eco-innovation (O'Hare, 2010). Level 1 focuses on more traditional 'tactical' DfE approaches, broadly consisting of analytical approaches to ecodesign and simpler approaches such as Design for $X$ (DFX) (Wrisberg et al., 2002). These are discussed in section 2.2.3.

\subsubsection{DfE and the product life cycle}

As discussed, a core underlying principle of DfE is the product life cycle. Figure 2.5 illustrates the typical life cycle of a product system and the generic flows of material within this cycle. Adopting a life cycle view is preferable as it allows a more holistic view of a product and its environmental impacts, avoiding shifting environmental burdens from one life cycle phase to another (Remmen et al., 2007).

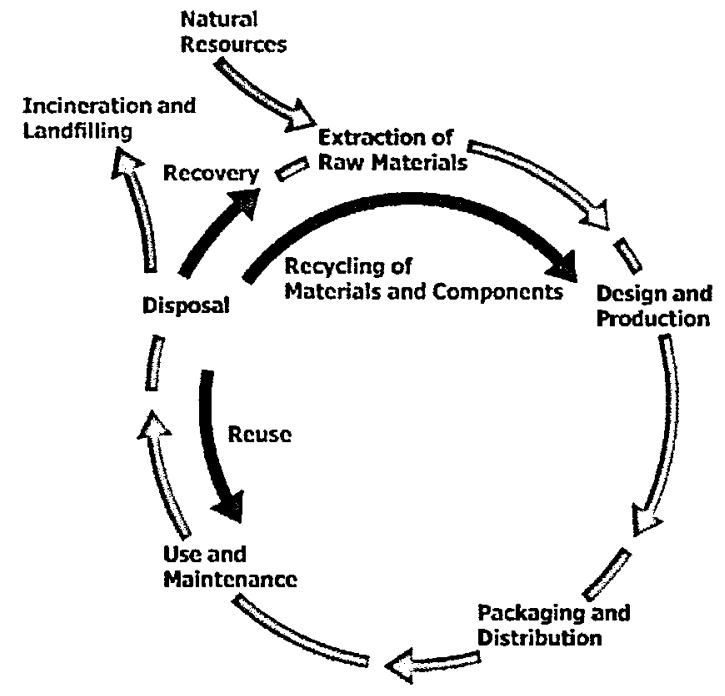

Figure 2.5: Product system life cycle (Remmen et al., 2007)

Common approaches to DfE are based on analytical approaches to understanding environmental impacts over the life cycle through Life Cycle Assessment (LCA), simplified forms of LCA or undertaking qualitative life cycle thinking activities (O'Hare, 2010). There is a more complex and subtle part of this problem, as conducting an environmental assessment requires some sense of environmental priorities, developing agreement on what environmental impacts need to be measured and how (Ryan, 2004). The outcome of an assessment will ensure the relevant practical actions are implemented to reduce environmental impacts (Delay-Saunders, 2006; Hauschild et al., 2004; Remmen et al., 2007). 
Designing a product, understanding its environmental impacts and implementing practical responses to reduce them creates an iterative loop, as shown in Figure 2.6. There is no limit to the number of times a design's environmental impacts can be assessed, practical actions taken to reduce them, leading to new designs, and so on.

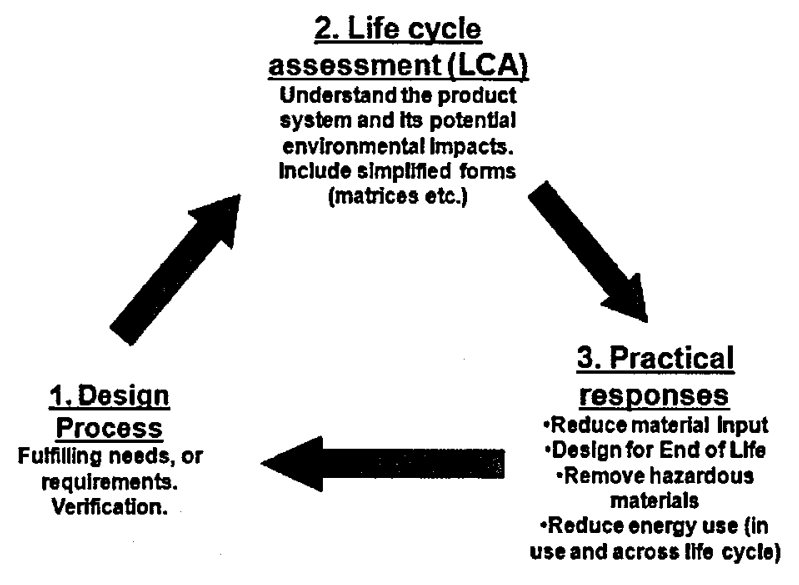

Figure 2.6: The iterative loop of DfE

Life Cycle Management (LCM) encompasses approaches to addressing sustainability over the life cycle of a business' products. LCM is not a specific tool or approach, rather a management system focused on a business' entire product value chain (a synonym for life cycle). There are specific tools that can be used within LCM, DfE being an example. Viewing DfE as an LCM tool helps focus the approach developed on the requirements of the business (Remmen et al., 2007).

\subsubsection{Non-technical considerations}

A strong theme in the DfE literature is the over-emphasis on the technical aspects of $\mathrm{DfE}$, focussing on developing tools. There appears to be a lack of consideration for the non-technical, or 'softer', aspects that need to be considered to effectively integrate environmental considerations into design decisions (Boks, 2006). Perhaps a symptom of this oversight is the observation that whilst there appears to be plenty of tools for DfE, evidence of these being successfully used in practice appears to be lacking (Boks, 2006; Lindahl, 2006; Luttropp and Lagerstedt, 2006).

Some studies have attempted to address this gap, pointing to the following nontechnical factors that need to be considered for effective DfE implementation:

- Understanding the company's needs for environmental information in design, developing a bespoke approach relevant to the identified 
requirements (Boks, 2006). Ammenberg and Sundin (2005) agree, noting there is no 'one size fits all' solution for DfE;

- Understanding the broader context of a company's product development processes (Baumann et al., 2002) and how the use of tools can be integrated into them (Lindahl, 2006);

- Creating a demand for DfE tools by setting strategic environmental objectives and communicating environmental requirements to design teams (Luttropp and Lagerstedt, 2006);

- Understanding the requirements of designers when it comes to using DfE tools, and design tools more broadly (Lindahl, 2006; Lofthouse 2006);

- Implementing processes for DfE to support the use of tools, for example incorporating consideration for DfE into design review gates (Boks, 2006);

- Senior management support (Delay-Saunders, 2006) and DfE champions who can facilitate cross-functional working that is often required for DfE (Veroutis and Fava, 1997);

- Considerations for organisational culture and how this may inhibit (or encourage) the uptake of DfE tools (Boks, 2006).

The literature is clear that one of the most important aspects of developing an approach to DFE is understanding the broader business context, in particular product development processes and the needs and demands for environmental information in design (Delay-Saunders, 2006; Lenox and Ehrenfeld, 1997; Wrisberg et al., 2002). However, there is a prevailing tendency to reduce DfE to a menu of strategies with an over-emphasis on the importance of data on environmental impacts, when what is required is a methodology for using this information to influence design decisions (Ryan, 2004).

\subsubsection{DfE tools}

This section reviews tools that can be applied for DfE. Baumann et al. (2002), DelaySaunders (2006) and Wrisberg et al. (2002) make classifications of DfE tools that can be developed, once the requirements for a tool have been understood. Types of tool can be broadly classified into approaches based on Life Cycle Assessment (LCA), simplified tools such as matrices and checklists, and generic DfE strategies, similar to the DFX approaches discussed by Maxwell and van der Vorst (2003). 


\subsubsection{Life Cycle Assessment (LCA)}

LCA is a 'methodological framework' for evaluating the potential environmental impacts of a product over its life cycle (Rebitzer et al., 2004, p.702). There are many reasons why a business might undertake LCAs, aside from integrating environmental considerations into design, common ones being for product comparisons or for understanding the environmental impacts of product systems in order to guide environmental strategy. One of the main features of LCA is that the assessment is done as a measurement per unit of function provided by a product (the functional unit).

A methodology for performing LCA studies is written into international standards (see BSI, 2006) and consists of 4 major steps, outlined in Figure 2.7.

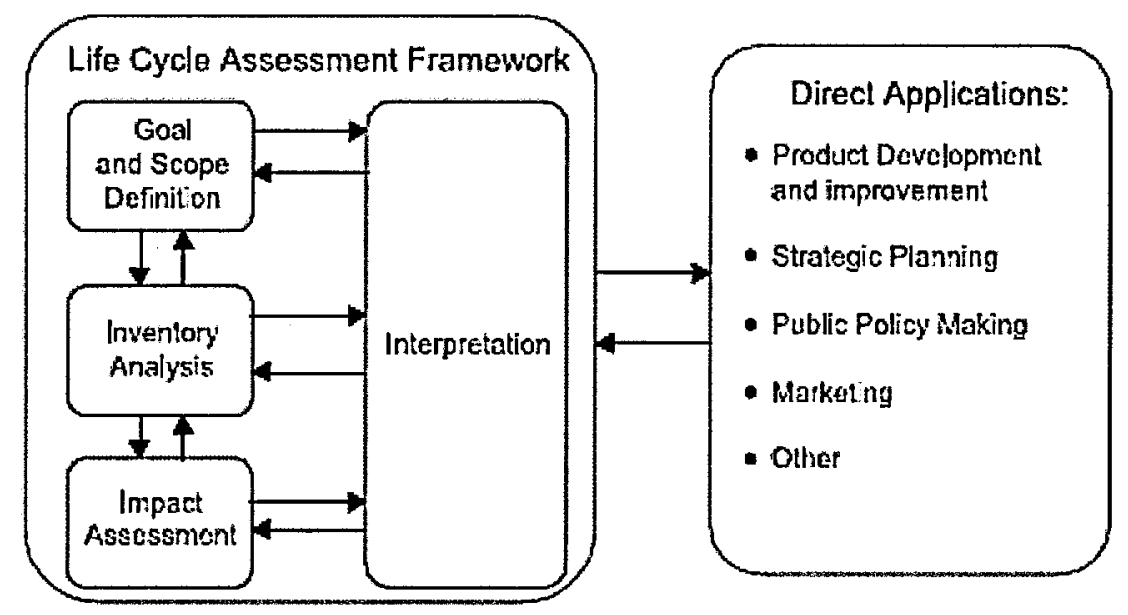

Figure 2.7: Phases and applications of LCA (Rebitzer et al., 2004)

Goal and scope definition, the first phase of LCA, outlines the objectives of the study and defines the functional unit and product system boundaries. Inventory analysis details the processes that take place across the life cycle of the product, modelling the inputs (consumption of resources) and outputs (waste flows and emissions), creating a Life Cycle Inventory ( $\mathrm{LCl}$ ). The inventory should include all energy and material flows within the limits stipulated by the goal and scope. Environmental impacts of this inventory are then evaluated through Life Cycle Impact Assessment (LCIA).

LCIA usually aggregates environmental impacts into several categories at various positions in the cause effect chain between an environmental aspect (something that interacts with the environment) and the real (or potential) environmental impact it causes. Evaluating potential impacts at the mid-point level (e.g. global warming potential, see Table 2.1) or the end-point level (i.e. damage to human health from global warming) are common approaches in LCIA, although there is a debate as to the validity of each approach. At the end-point level there are significant uncertainties whilst the relevance of results is sometimes judged to be questionable at the mid-point 
level (Bare et al., 2000). LCIA is also complicated due to its inevitable dependence on value judgements (Finnveden, 2000; Pennington et al., 2004).

Table 2.1: Common mid-point environmental impact categories (Wrisberg et al., 2002;

Baumann and Tillman, 2004)

\begin{tabular}{|l|l|}
\hline Extraction of abiotic resources & Extraction of biotic resources \\
\hline Land use & Climate change \\
\hline Stratospheric ozone depletion & Photo-oxidant formation \\
\hline Human toxicity & Eco-toxicity \\
\hline Acidification & Nutrification \\
\hline
\end{tabular}

Interpretation in LCA introduces an iterative element to the assessment that covers all stages, being used to evaluate and communicate the environmental impacts identified by the study. In interpretation, it is sought to communicate the sometimes complex results of an LCA study in a manner that can be understood and acted upon by decision makers.

Common criticisms of LCAs are that they are often seen to be expensive, time consuming and provide results that are difficult to interpret (Baumann et al., 2002). In a lot of cases a simplified methodology is required, as the effort to complete a full LCA is not justified by the requirement. This is especially relevant to the use of LCA during design (Christiansen, 1997; Graedel and Allenby, 1995). Delay-Saunders (2006, p.66) criticises the use of LCA in its purest form as a decision making tool during design for these reasons, stating that LCA in this context is 'both valuable and useless'. As a result, LCA in its applied form as DfE is usually simplified (sometimes the term streamlined is used, they are considered synonymous). Popular approaches to simplification include limiting the areas of the product system being studied to selected life cycle stages, limited approaches to impact assessment or the use of qualitative data (Christiansen, 1997). Further simplifications can be made in the development of alternative analytical approaches that are more based on life cycle thinking than LCA, such as matrices and checklists.

\subsubsection{Matrices and checklists}

Graedel and Allenby (1995) developed the use of matrices as a DfE tool and another matrix approach was also introduced by Brezet and Van Hemel (1997). Although there are variations in the approach, the overarching principle of DfE matrices is the same. A simplified life cycle approach is developed by listing the life cycle stages and environmental impacts in a matrix format similar to that shown in Figure 2.8. 


\begin{tabular}{|c|c|c|c|c|}
\hline & & $\begin{array}{l}\text { Material cycle } \\
\text { (input/output) }\end{array}$ & $\begin{array}{c}\text { Energy use } \\
\text { (input/output) }\end{array}$ & $\begin{array}{c}\text { Toxic emissions } \\
\text { (output) }\end{array}$ \\
\hline \multicolumn{2}{|c|}{$\begin{array}{l}\text { Production and supply of } \\
\text { materials }\end{array}$} & & & \\
\hline \multicolumn{2}{|c|}{ Manufacture } & & & \\
\hline \multicolumn{2}{|c|}{ Distribution } & & & \\
\hline \multirow{2}{*}{ Use } & operation & & & \\
\hline & servicing & & & \\
\hline \multirow{2}{*}{ End of life } & recovery & & & \\
\hline & disposal & & & \\
\hline
\end{tabular}

Figure 2.8: MET matrix (Brezet and Van Hemel, 1997)

From Wrisberg et al. (2002), the methodological approach used in LCA can be applied to develop the matrix, defining the goal and scope and performing inventory and impact assessment steps. Although significantly less detail is required and the matrix is used as a simple means of recording the outputs of the assessment. The benefit of the matrix approach is that it provides a quick, holistic life cycle picture. There is also relative flexibility in the type of information deployed, from quantitative to qualitative. If desirable, the matrix can be used for simple aggregations to represent the environmental impacts as a single score (Graedel and Allenby, 1995). Although matrices can deploy quantitative information, they are still limited to being largely descriptive (Wrisberg et al., 2002).

Wrisberg et al. (2002) introduce checklists as another common DfE approach, being simply a set of questions or points which ensure that the designer has made consideration for all of the required elements of the design, from an environmental perspective. Checklists can also include things to avoid. The benefits of checklists are similar to those of a matrix, especially in the flexibility of approach provided, as the effectiveness of the assessment is essentially dependant on the nature of the questions posed and the information required to answer them. Checklists can also be manipulated in order to make information more presentable or relevant to decision makers, an example being the eco-strategies wheel developed by Brezet and Van Hemel (1997).

\subsubsection{Generic DfE strategies}

One of the most common approaches to DfE is through the use of generic DfE strategies (Maxwell and van der Vorst, 2003). These strategies assume an environmental benefit through their deployment, instead of using analytical approaches to improve environmental performance. There are numerous sets of general principles in the literature, summarised from Brezet and Van Hemel (1997) in Table 2.2. 
Table 2.2: Summary of generic DfE strategies (Brezet and Van Hemel, 1997)

\begin{tabular}{|c|l|}
\hline Select low impact materials & $\begin{array}{l}\text { Where practical avoid use of hazardous substances and metals with } \\
\text { high environmental impacts from production. Focus on using } \\
\text { recycled and recyclable materials and the avoidance of mixing } \\
\text { materials in a way in which they cannot be separated and recycled. }\end{array}$ \\
\hline Reduce material use & $\begin{array}{l}\text { May reduce the environmental impacts of materials production and } \\
\text { impacts during use. }\end{array}$ \\
\hline Optimise production & $\begin{array}{l}\text { Synonymous with cleaner production, driven by the adoption of a } \\
\text { certified environmental management system. Emphasis should be } \\
\text { placed on the selection of cleaner production techniques as opposed } \\
\text { to the clean-up approach. Alternatives should be sought to } \\
\text { particularly environmentally damaging processes. }\end{array}$ \\
\hline Optimise distribution & $\begin{array}{l}\text { Including the packaging (which should be considered as a product in } \\
\text { its own right, with a life cycle) the mode of transportation and } \\
\text { logistics. }\end{array}$ \\
\hline Optimise lifetime and re-use & $\begin{array}{l}\text { In particular for products that consume energy during use, e.g. } \\
\text { household appliances and cars. }\end{array}$ \\
\hline Optimise end of life & $\begin{array}{l}\text { By prolonging the use of the product, through maintenance, design } \\
\text { for durability and modular design, maximum service is provided with } \\
\text { the amount of materials used. Can be Driven by a PSS service } \\
\text { based business model. }\end{array}$ \\
\hline & $\begin{array}{l}\text { Encouraging re-use, waste reduction, recycling and reducing waste } \\
\text { to landfill. }\end{array}$ \\
\hline
\end{tabular}

It is generally accepted that the adoption of generic DfE strategies leads to environmental benefits, although caution should be applied. Some strategies focus on one phase of the life cycle only, which could shift environmental burdens to another (Ryan, 2004).

\subsubsection{Examples of DfE within industry}

This section reviews examples of DfE within industry taken from the academic literature. The review is not intended to be exhaustive, and instead aims to provide a flavour of how DfE has been practically implemented within businesses to support preceding sections that have provided a theoretical overview of the subject area. The following sections provide examples of DfE drawn from the following industrial sectors:

- Aerospace;

- Automotive and transport;

- Information Technology (IT);

- Other examples.

This review is limited by the fact that few businesses publish details of their DfE activities within the academic literature. 


\subsubsection{Aerospace}

Within the aerospace industry there are DfE examples at aircraft manufacturers Airbus and Boeing and engine manufacturer Pratt and Whitney.

Delay-Saunders (2006) presents a thesis focused on integrating environmental considerations into design at Airbus. The approach taken characterised design processes to identify opportunities for an environmental influence in design. Using knowledge of the decision-making context, DfE solutions were developed which would provide the relevant information at each decision point. The environmental intervention points are summarised in Figure 2.9, which also highlights which life cycle environmental impacts are addressed at each phase of the design process. Figure 2.9 also highlights how there is a greater potential for environmental improvements earlier in the design cycle, as discussed previously.

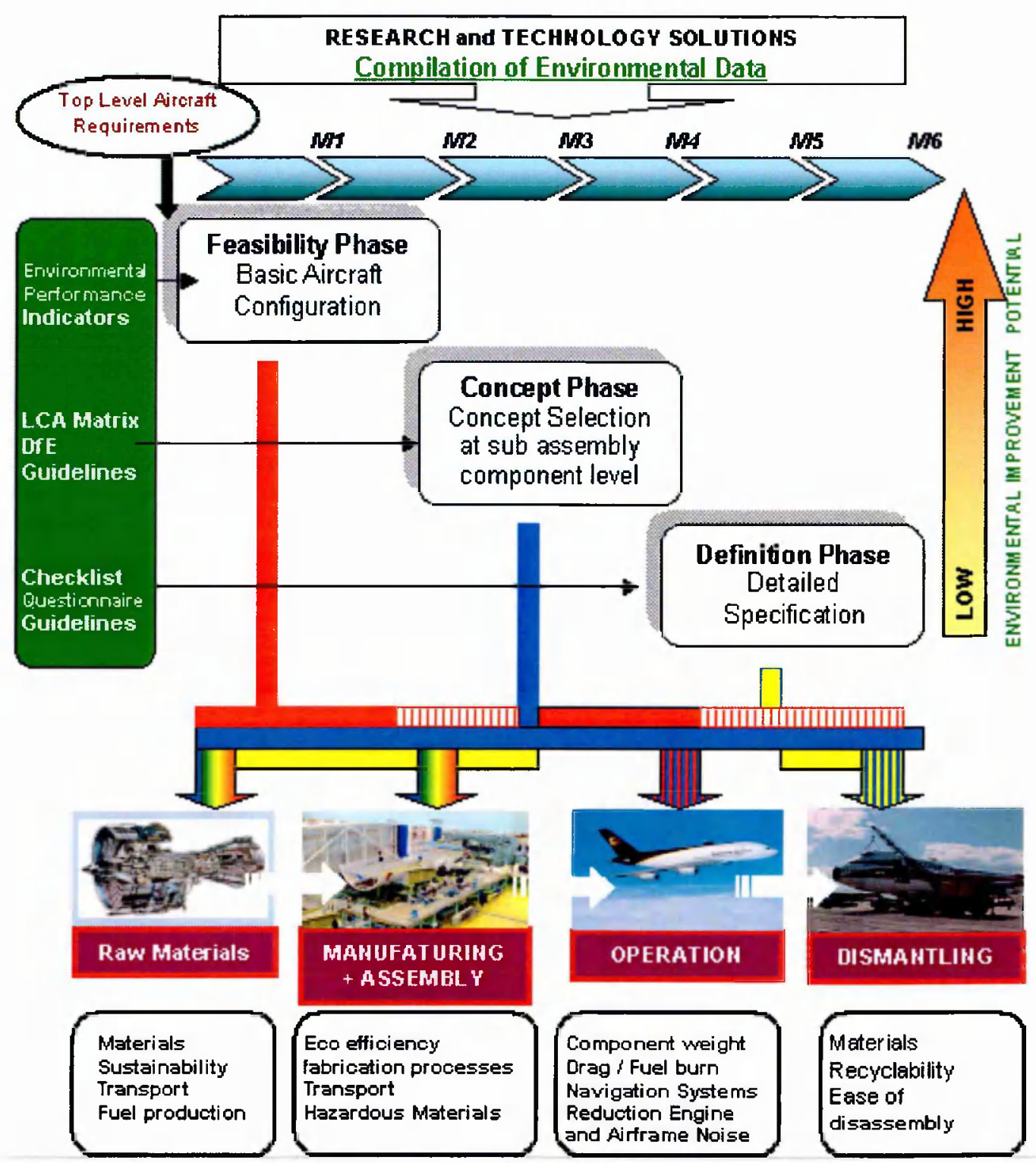

Figure 2.9: Environmental effects of design decisions at Airbus (Delay-Saunders, 2006) 
Weinberg and Eagan (1997) present an approach to DfE at Boeing, applying a simple matrix similar to the one shown in Figure 2.8, with a specific focus on reducing environmental impacts from over the life cycle of manufacturing processes. A later publication by Boeing (2006) gives detail of a more thorough view of DfE within the business. Four strategies for DfE listed are:

- Improvements in fuel efficiency per passenger km flown;

- The reduction in perceived noise from aircraft operations;

- Improvements in resource efficiency and waste production in manufacturing through the deployment of lean principles;

- Aircraft end of life considerations and improvements in recycling.

The article does not mention the use of any specific analytical approaches to DfE, merely detailing the adoption of generic DfE strategies and how these are seen to compliment cost reduction efforts.

Swarr et al. (1999) present the development of an approach to integrate environmental considerations into design through the use of a streamlined life cycle assessment at Pratt \& Whitney, who manufacture gas turbine aero engines. The DfE approach was developed through consultation with the design community to ensure it was relevant to the needs of designers. This highlighted how designer's desks were already overloaded and that if a DfE metric was to be included along with other requirements it would have to be something specific and compatible with other functional aspects of the design to allow for trade-offs. The conclusion was that the best approach to DfE was to find a way to incorporate environmental considerations into design in a manner consistent with existing design processes.

\subsubsection{Automotive and transport industries}

Within the automotive and transport industries there are DfE examples at automotive manufacturers Ford and Toyota and train manufacturer Bombardier.

Schmidt and Taylor (2006) introduce Ford of Europe's (FoE) efforts to incorporate Product Sustainability Indicators (PSIs) into their product development processes through a streamlined life cycle approach. Figure 2.10 gives an overview of the indicators used by Ford's PSI system, which were built in to a simple IT based tool for use by product developers. Schmidt and Taylor argue that environmental considerations could only be properly integrated into product development if the method is tailored to the company specific situation and through a voluntary approach with senior management commitment. To do so without these pre-requisites would 
'significantly increase the costs of applications without adding any value for the environmental, social or economic performance of the vehicles' (Schmidt and Taylor, 2006, p.9). The tailoring of approach included environmental impact categories, with a hybrid impact assessment methodology being applied. Environmental impacts of strategic interest such as Global Warming Potential and Air Quality (Photochemical Creation Potential) were aggregated to the mid-point level. However for toxicity effects, hazardous substances were dealt with at an inventory level. A unique indicator was developed for resource use as all other assessment methods were deemed to be inadequate.

\begin{tabular}{|c|c|c|c|}
\hline & Indicator & Metric/method & Driver for inclusion \\
\hline \multirow[t]{5}{*}{$\begin{array}{l}\text { Environ } \\
\text { mental } \\
\& \\
\text { Health }\end{array}$} & $\begin{array}{l}\text { Life Cycle } \\
\text { Global Warming } \\
\text { Potential }\end{array}$ & $\begin{array}{l}\text { Greenhouse emissions along the life cycle (vehicle } \\
\text { production, driving } 150000 \mathrm{~km} \text { based on EUCAR } \\
\text { agreement [12], end-of-life) } \\
\text { - part of an LCA according to ISO14040 }\end{array}$ & $\begin{array}{l}\text { Carbon intensity is the main } \\
\text { strategic issue in automotive } \\
\text { industry }\end{array}$ \\
\hline & $\begin{array}{l}\text { Life Cycle Air } \\
\text { Quality Potential }\end{array}$ & $\begin{array}{l}\text { Summer Smog Creation Potential POCP) along the } \\
\text { same life cycle (VOCs, NO) - part of an LCA according } \\
\text { to ISO14040 }\end{array}$ & $\begin{array}{l}\text { Non- } \mathrm{CO}_{2} \text { air quality issues have to } \\
\text { be monitored for trade-offs }\end{array}$ \\
\hline & $\begin{array}{l}\text { Sustainable } \\
\text { Materials }\end{array}$ & Recycled \& natural materials related to all polymers & Resource Scarcity \\
\hline & $\begin{array}{l}\text { Restricted } \\
\text { Substances }\end{array}$ & $\begin{array}{l}\text { Vehicle Interior Air Quality / allergy-tested interior, } \\
\text { management of substances along the supply chain ( } 15 \\
\text { point rating) }\end{array}$ & $\begin{array}{l}\text { Substance risk management is } \\
\text { key }\end{array}$ \\
\hline & $\begin{array}{l}\text { Drive-by-exterior } \\
\text { Noise }\end{array}$ & Drive-by exterior Noise $=\mathrm{dB}(\mathrm{a})$ & Main societal concern \\
\hline \multirow[t]{2}{*}{ Social } & Safety & $\begin{array}{l}\text { Including number of all EuroNCAP stars (see note } \\
\text { under acronyms regarding EuroNCAP) }\end{array}$ & Main direct impact \\
\hline & $\begin{array}{l}\text { Mobility } \\
\text { Capability }\end{array}$ & $\begin{array}{l}\text { Mobility capacity (luggage compartment volume plus } \\
\text { weighted number of seats) related to vehicle size }\end{array}$ & $\begin{array}{l}\text { Crowded cities (future: diversity - } \\
\text { disabled) }\end{array}$ \\
\hline $\begin{array}{l}\text { Eco- } \\
\text { nomics }\end{array}$ & $\begin{array}{l}\text { Life Cycle } \\
\text { Ownership } \\
\text { Costs }\end{array}$ & $\begin{array}{l}\text { Vehicle Price plus } 3 \text { years fuel costs, maintenance } \\
\text { costs, taxation minus residual value (note: for } \\
\text { simplification reasons costs have been tracked for one } \\
\text { selected market Life Cycle Costing approach using } \\
\text { discounting) }\end{array}$ & Customer focus, competitiveness \\
\hline
\end{tabular}

Figure 2.10: Ford's Product Sustainability Indicators (Schmidt and Taylor, 2006)

Toyota's Eco-VAS (Eco Vehicle Assessment System) (Figure 2.11) aims for improvements in the following strategic areas, based on the setting of targets by chief engineers:

- Fuel efficiency;

- Exhaust emissions;

- External vehicle noise;

- Reduction of environmental impact throughout the entire life cycle of vehicle use;

- Improved recyclability;

- Reduction in the use of substances of concern.

The targets are monitored through a 'comprehensive environmental impact assessment system' based on LCA (Toyota, 2009). This includes the flow down of requirements for environmental information through the supply chain. 


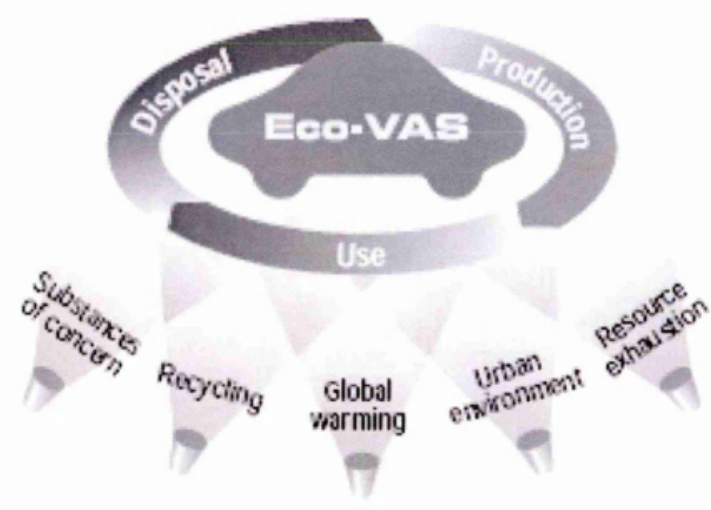

Figure 2.11: Toyota Eco-VAS (Toyota, 2009)

Lagerstedt and Luttropp (2006) introduce the development of DfE guidelines at Bombardier transportation. The paper introduces the '10 Golden Rules' of Ecodesign, which are similar to the generic DfE strategies introduced in Section 2.2.3.3. The paper details how the rules were tailored for Bombardier transportation into a DfE guidance document for use by product developers. The guidance document can best be described as a DfE checklist, as there is no evidence of any form of analytical approach to measure and reduce environmental impacts.

\subsubsection{IT industry}

Within the IT industry there are DfE examples at Intel, Apple and Hewlett Packard.

Brady and McManus (2003) introduce DfE at Intel, which is based on generic strategies and setting improvements targets for energy efficiency, the use of hazardous materials and the production of waste. Initially the focus was on achieving improvements in manufacturing although this was then extended to product-focused targets. No detail is given on the relevance of the DfE approach to the product development processes, although it is commented that due to the rapid development of the technologies, there is a very short window for DfE improvements. There is no mention of analytical approaches to DfE.

Apple has a product design philosophy that includes DfE principles (Apple, 2009). Areas of environmental focus are listed as improving the energy efficiency of products, reductions in the use of hazardous materials and improvements in the recyclability of their products and packaging. Hewlett Packard (HP) adopt a similar approach (HP, 2009). As appears to be the case at Intel, both Apple and HP do not report any use of analytical approaches to DfE or the inclusion of systems considerations in the integration of DfE principles. It is likely that this finding is due to the lack of available literature. 
Ryan (1999) provides an interesting commentary on possible shifts in approaches to DfE within the IT industry. Ryan proposes a shift from the current approach of using generic DfE strategies to exploiting new IT technologies that can deliver significant environmental improvements. One example is reducing business travel by promoting the use of video conferencing for meetings.

\subsubsection{Other examples}

Other examples of DfE were found in the literature at Lucent Technologies, Motorola and Black \& Decker.

Donnelly et al. (2006) introduce DfE at Lucent Technologies, a telecommunications business. The approach applied the template provided by ISO14001 (see BSI, 2004) identifying environmental aspects, assessing their significance and establishing objectives using a PDCA (Plan-Do-Check-Act) cycle. This was applied to the product development process, resulting in the identification of touch points for the consideration of environmental effects during product development, as shown in Figure 2.12. Different tools tailored for each specific touch point were identified.

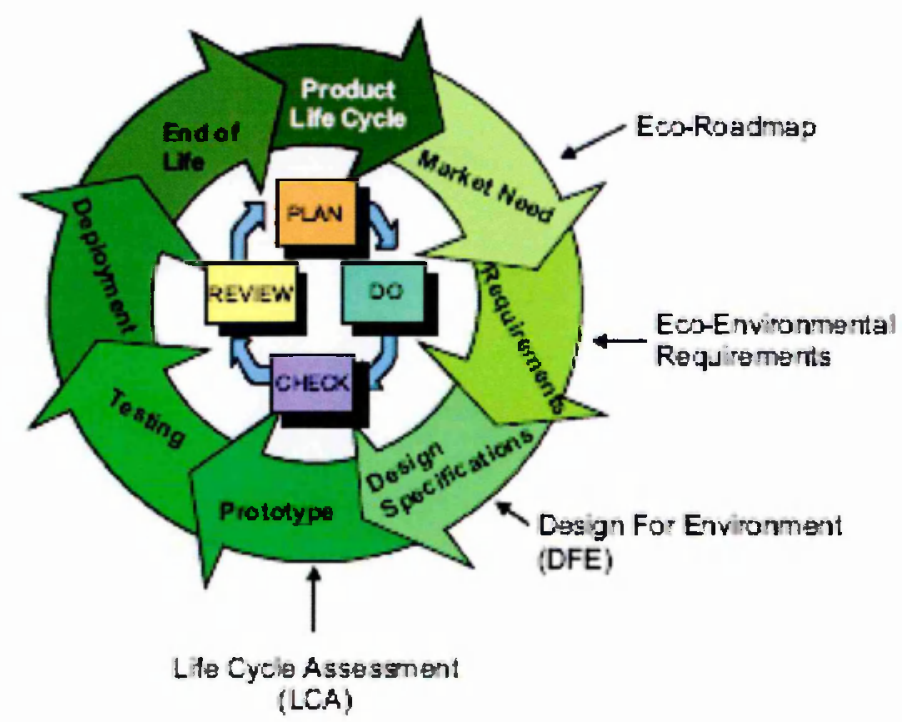

Figure 2.12: Lucent Technologies PBEMS touch points (Donnelly et al., 2006)

The tools deployed ranged from an 'eco-roadmap' used to highlight short and long term environmental drivers (including legislative changes), listing environmental considerations in product requirement documents, applying generic DfE principles and using LCA to identify significant environmental effects. The approach was not only developed bearing in mind the business' product development processes, but also the wider business planning and organisation. Doing this as part of a management system 
ensured that the approach adopted was fully integrated in to the business, and objectives and targets were set for improvement, and achieved.

The Lucent approach highlighted several common barriers to the implementation of DfE approaches within a business. In particular, the difficulty in achieving a culture change to progress from an understanding of facility environmental issues to considering the whole product life cycle. They conclude that the success of implementing DfE into a business 'relies on the efficient integration' of DfE and product development (Donnelly et al., 2006, p.1365).

Hoffman (1997) introduces DfE approaches used at Motorola, a large electronics company. DfE is set within the context of the product development process at Motorola, which is based on 3 steps: concept development, detail design and prototype manufacture. A specific DfE approach is developed to match the information available at each step of the design process. In concept development, a matrix-based simplified life cycle assessment approach is used, which is based on a series of questions. Answers to the questions are assigned scores to produce a semi-quantitative assessment. A more quantitative approach is used during detail design, applying a scoring system to evaluate eight criteria: energy, mass, recycled material content, recyclability, number of materials, toxicity of materials, disassembly time and commodity value. Scores for each category are assessed for each design, then weighted and aggregated to provide a total score. A full LCA is the proposed DFE tool at the prototype development stage. Hoffman (1997) highlights the importance of developing an approach relevant to each stage of the design process. Doubts over the suitability of using a full LCA in design are also expressed.

Fitzgerald et al. (2005) introduce the development of a DfE approach at Black \& Decker, applying a simple semi-quantitative set of guidelines and checklists. The guidelines are based on strategic environmental drivers set by the business. This led to the development of the following points of focus for DfE:

- 'Flagged' material use in products;

- Total product and packaging mass (quantitative value);

- 'Flagged' material generated in manufacturing process;

- Recyclability and disassembly rating (semi-quantitative value);

- Energy consumption during use (quantitative value).

These points were then built into a DfE matrix, accompanied by checklists for considering innovation and regulatory compliance. The paper details how these guidelines and checklists were integrated in to the stage-gate product development process used at Black \& Decker, with the score card and checklist being applied at the 
concept, detailed and final design reviews. Fitzgerald et al. (2005) also discuss difficulties in developing DfE approaches based on LCA.

\subsubsection{Summary and conclusions}

The purpose of this section was to review the academic literature on the topic of Design for Environment (DfE) to gain insights into how an approach to integrating environmental considerations into design decisions could be developed at Rolls-Royce. DfE has been defined as a 'systematic process by which firms design products in an environmentally conscious way' (Baumann et al., 2002, p.413), effectively seeking to merge the product development cycle in businesses with the physical life cycle of a product. The context for DfE was set among the different levels of environmental design, from strategic considerations of business model to the more traditional focus of DfE on eco-innovation and tactical eco-design strategies, typically involving the use of analytical techniques. The importance of organisational considerations in developing a DfE approach were discussed, in particular the need to understand the demand for environmental information in design, establish environmental priorities and provide a methodology to support the use of DfE tools. Typical DfE tools were then described, ranging from relatively complicated LCAs, to simplified forms of LCA, matrices, checklists and generic DfE strategies. These are used to assess the environmental impacts of products to drive practical steps for reducing these impacts, creating an iterative improvement cycle. A review of examples of DfE implemented within business was then provided to support the theoretical background.

Concluding this section, several findings can be used to guide research direction:

- It is clear that developing a thorough understanding of the business and design context is an integral part of developing any approach to integrating environmental considerations into design decisions.

- A bespoke approach relevant to this context will then be required, developing solutions tailored to the organisational context and individual steps within the design process. This point was emphasised from the DFE examples at Airbus, Lucent Technologies and Motorola.

- Setting the level of environmental improvement that is to be achieved is also important. Typical approaches to DfE focus on product improvement (level 1) and eco-innovation (level 2) improvements.

- Setting environmental priorities, in terms of what environmental impacts are addressed and how these are measured and assessed with the use of tools, 
is also a fundamental part of developing any approach. This should then lead to the appropriate practical responses in design.

- Any approach must be based on some understanding of the environmental impacts of products, although for practical reasons full LCAs are not suitable as a design tool. DfE tools are generally based on simplified approaches to LCA, matrices or generic strategies and checklists. Schmidt and Taylor (2006) provided a good example of how a simplified form of LCA was developed incorporating an environmental assessment method relevant to the needs of the organisation.

- Non-technical aspects need to be considered for effective DfE implementation, including how organisational culture may encourage, or inhibit, the uptake of DfE.

- The DfE example from Pratt \& Whitney is particularly interesting, being a gas turbine aero engine manufacturer similar to Rolls-Royce. Swarr et al. (1999) concluded that any DfE approach developed should be compatible with existing design processes and ways of working. It was also concluded that any DfE metrics had to be related to something specific that could be compared with other design requirements to allow for trade-offs. These conclusions are relevant to integrating environmental considerations into design decisions at Rolls-Royce.

Building on these findings, the next section continues to investigate the research theme for this chapter by providing more detail on the business and design contexts for this research.

\subsection{Understanding the context}

This section provides the detail of the business and design contexts within RollsRoyce, as an integral part of understanding how environmental considerations could be integrated into design decisions in Rolls-Royce. To provide a high level overview of the Rolls-Royce business context, section 2.3.1 introduces the concepts of organisational culture and structure, describes the researcher's interpretation of the organisational structure and culture at Rolls-Royce, and discusses how this might influence any approach to integrating environmental considerations into design decisions. Following on from this discussion, Section 2.3.2 provides an overview of design processes at Rolls-Royce, including the strategic design context, standard design framework and tools used within design. Section 2.3 .3 provides a summary and conclusions. 


\subsubsection{Organisational culture and structure}

Organisational culture refers to a 'pattern of basic assumptions' that influence how an organisation works (Schein, 1990, p.111). Organisational cultures can encompass (from Handy, 1999):

- How work is organised and planned;

- Expected behaviours in managers and workers;

- How decisions are made, for example by individuals or groups;

- Remuneration;

- The formality or flexibility of working arrangements, including the adherence to (or absence of) rules and procedures;

- The type of people the organisation employs.

Cultures are also typically reflected in a 'structure and set of systems', being strongly linked to how organisations are designed (Handy, 1999, p.181).

Harrison (1972) offers a conceptual framework describing the different types of organisational culture, Handy (1999) noting consistencies with a similar framework offered by Mintzberg (1989). Table 2.3 provides an overview of Harrison's conceptual framework, with links to organisational structures from Handy (1999). 
Table 2.3: Conceptual framework of organisational cultures (Handy, 1999; Harrison, 1972)

\begin{tabular}{|c|c|c|}
\hline Culture & Description & Structure \\
\hline Power & $\begin{array}{l}\text { Pictured as a web, this culture relies upon } \\
\text { a central authority figure and is commonly } \\
\text { found in small entrepreneurial } \\
\text { organisations: } \\
\text { - Control is exercised by the centre } \\
\text { or key individuals. } \\
\text { - Adaptable to change. } \\
\text { - Can limit the size of } \\
\text { organisations. }\end{array}$ & \\
\hline Role & $\begin{array}{l}\text { Pictured as a Greek temple, this culture is } \\
\text { controlled by functions and procedures, } \\
\text { which could be described as a } \\
\text { bureaucracy, controlled by managers at } \\
\text { the top: } \\
\text { - Job descriptions are more } \\
\text { important than the person who } \\
\text { does the job. } \\
\text { Workers are expected to do their } \\
\text { job and nothing more. } \\
\text { Rules are the main method of } \\
\text { influence. } \\
\text { Thrives on stability, although slow } \\
\text { (and hence susceptible) to } \\
\text { change. }\end{array}$ & \\
\hline \multirow[b]{2}{*}{ Task } & $\begin{array}{l}\text { Structured around projects, pictured as a } \\
\text { net, focussed on getting the job done: } \\
\text { - Typical of 'matrix organisations'. } \\
\text { - Influence resides at intersections } \\
\text { of the net. }\end{array}$ & \\
\hline & $\begin{array}{l}\text { - Team needs and objectives take } \\
\text { precedence over the } \\
\text { requirements and contributions of } \\
\text { individuals. } \\
\text { - Common in competitive markets } \\
\text { where flexibility is required. }\end{array}$ & \\
\hline Person & $\begin{array}{l}\text { Focussed on the needs of individuals, } \\
\text { pictured as a cluster with minimal formal } \\
\text { structure: } \\
\text { - The organisation only exists to } \\
\text { serve the needs of individuals. } \\
\text { - Uncommon, as not many } \\
\text { organisations exist without a } \\
\text { common objective. } \\
\text { - Influence is shared amongst } \\
\text { members. } \\
\text { - Examples include partnerships. }\end{array}$ & \\
\hline
\end{tabular}

Harrison's framework is obviously simplistic. In reality it is difficult to define a culture, as it only exists in terms of the thoughts or feelings of those who perceive it (Handy, 1999). However, the framework does have an intuitive logic.

There is unlikely to be one universal culture that describes an organisation. Successful organisations pick the most appropriate cultures, structures and systems for the situations and tasks at hand, integrating them into the organisational whole (Handy, 
1999). Mintzberg (1989) refers to this integration of cultures and structures into the organisation as 'configuration'. Table 2.4 provides a summary of the influencing factors and task types that could determine the appropriate organisational culture, structure and systems in any given situation.

Table 2.4: Influencing factors and task types that could determine culture (Handy, 1999)

\begin{tabular}{|l|l|}
\hline \multicolumn{1}{|c|}{$\begin{array}{c}\text { Influencing factors (related to } \\
\text { the organisation) }\end{array}$} & \multicolumn{1}{|c|}{ Task types } \\
\hline $\begin{array}{l}\text { History and ownership } \\
\text { Size }\end{array}$ & $\begin{array}{l}\text { Steady state } \\
\text { Innovation }\end{array}$ \\
Technology & Breakdown \\
Goals and objectives & Policy \\
The environment & \\
The people & \\
\hline
\end{tabular}

As discussed in the previous section, organisational culture is an important nontechnical factor that needs to be considered in the development of a DfE approach. Hence, it is important to develop an interpretation of the organisational structure and culture at Rolls-Royce to investigate into how environmental considerations could be integrated into design decisions in Rolls-Royce.

Beginning with structure, Figure 2.13 provides a simplified interpretation of RollsRoyce's business structure.

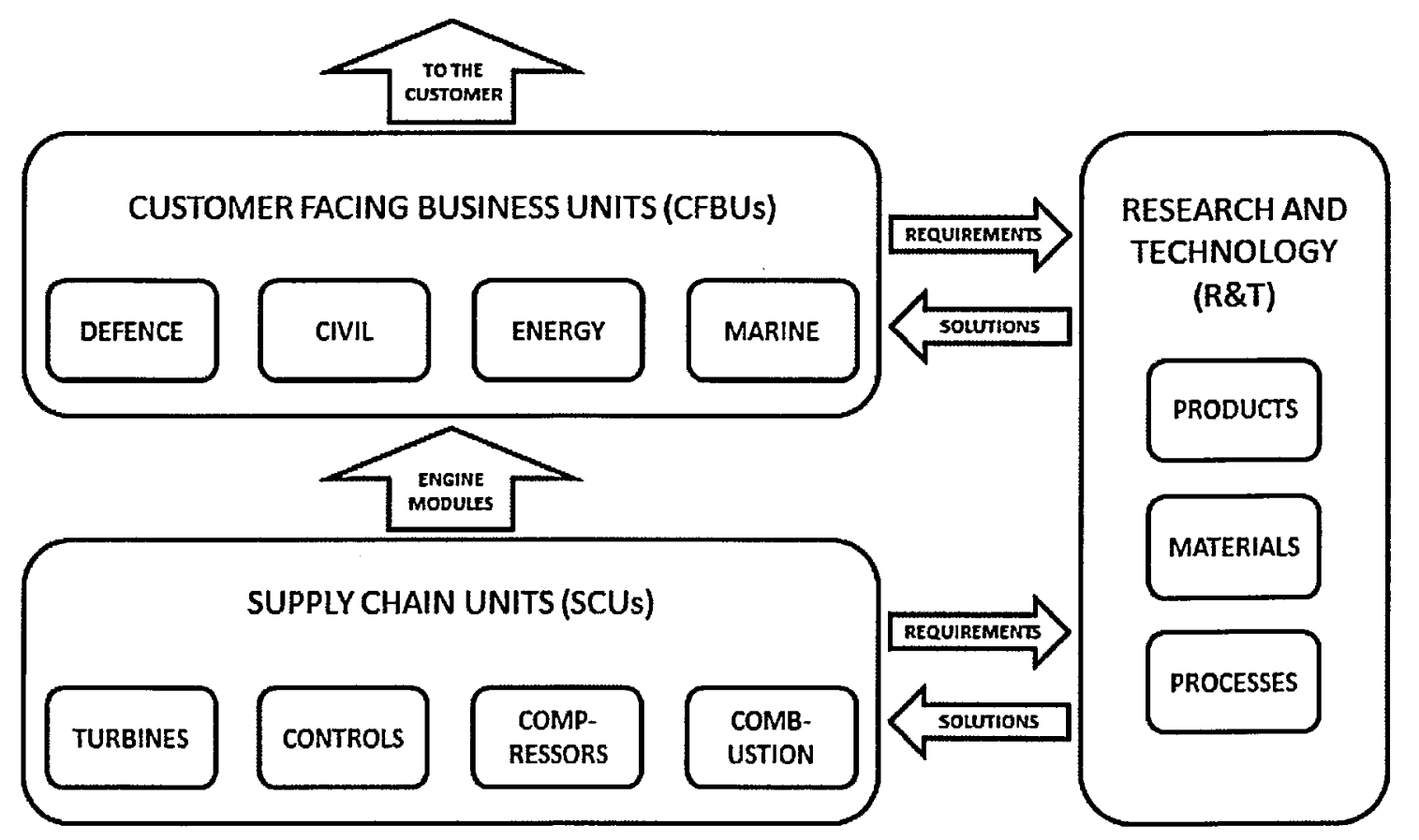

Figure 2.13: Simplified Rolls-Royce business structure (for illustration only 4 SCUs are shown)

The group is divided into Customer Facing Business Units (CFBUs) aligned to its main markets: Defence Aerospace, Civil Aerospace, Marine and Energy (which also includes the Nuclear business). Each of the CFBUs is structured around programmes, each 
one being responsible for the supply of a specific product type to customers. These programmes do not manufacture any parts, but are responsible for integrating subassemblies, produced in modules manufactured by Supply Chain Units (SCUs), into a complete product. SCUs are broadly structured around the different parts of a gas turbine (compressors, rotatives, combustion, turbines, and so on), specialising in manufacturing one particular type of engine module or set of components. A separate Research and Technology (R\&T) function is responsible for providing the necessary technologies required for continued product improvements, including developing new product concepts for CFBUs and materials and manufacturing processes for SCUs.

Figure 2.13 reflects a typical task orientated culture, as described in Table 2.3, which is common within large aerospace organisations (Handy, 1999). However, this structure only reflects one aspect of the organisational philosophy at Rolls-Royce. Superimposed upon this task orientated structure is a set of governance processes. These define how programmes are executed, setting processes, policies and standards for all related activities, for example engineering design. Governance processes are controlled by central functions that operate at a corporate level across the whole business. Each CFBU also operates its own set of central functions aligned to those at a corporate level, which are effectively responsible for the local execution of corporate processes. Corporate level functions are responsible for ensuring consistency of approach across different CFBUs.

Whilst being structurally task orientated, this organisational philosophy means that there is also a strong role culture within Rolls-Royce. Several influencing factors indicate why such an organisational philosophy has developed:

- Nature of the products manufactured by Rolls-Royce: Safety is critical and products have to satisfy thorough airworthiness checks and certification requirements. Part of this is demonstrating that products have been designed and manufactured with adherence to a process, as this helps to ensure the appropriate level of quality. These products are also complicated and rely on the use of advanced engineering techniques for development and manufacture. As a result, significant time, effort and validation are required before a suitable level of technological maturity is reached, which also requires robust processes.

- Organisational size: Large organisations, like Rolls-Royce, typically require some role type culture to guide the working of large and sometimes geographically dispersed groups.

- Age and history: Rolls-Royce is an old and well established organisation and has a distinctly conservative feel. This conservatism places a strong 
emphasis on adherence to process. Improvements generally occur incrementally and it takes time for new ideas and ways of working to become embedded into standard practice. A good expression of this conservatism is the external communications policy at Rolls-Royce, which explicitly seeks to avoid media attention.

Integrating environmental considerations into design decisions within an organisational philosophy that exhibits a role culture suggests that developing an approach consistent with existing processes is required. Developing an approach that requires changes to standard ways of working is unlikely to be successful within an organisation like RollsRoyce. Any approach has to be integrated into current ways of working, and importantly, into existing ways of making design decisions. This conclusion is consistent with Swarr et al. (1999), who also noted that any approach to integrating environmental considerations into design decisions at Pratt \& Whitney (another gas turbine aero engine manufacturer) had to be consistent with existing processes.

In order to take such an approach it is necessary to understand the existing organisational design processes within which environmental considerations need to be integrated. This is provided in the next section through a review of design at RollsRoyce. Conclusions of the review of academic literature on the topic of DfE also emphasised the importance of understanding the design context.

\subsubsection{Overview of design processes}

This section provides an overview of the Rolls-Royce design process. The strategic design context is defined by considering how the overall organisational structure influences the structure of design processes. The following sections discuss the standard design framework used within CFBUs and SCUs, followed by brief details of the tools applied within the standard design framework.

\subsubsection{Strategic design context}

To understand the Rolls-Royce design process, it is first necessary to refer back to the organisational structure shown in Figure 2.13. This structure defines three distinct parts of the organisation, with each having its own set of design responsibilities:

1. Customer Facing Business Units (CFBUs): CFBUs are structured into programmes each being responsible for delivering a particular product to customers. Programmes have overall responsibility for a product design, 
integrating modules and components designed at a detailed level into a whole product through systems design and systems engineering techniques.

2. Supply Chain Units (SCUs): SCUs are structured around the different parts of a gas turbine engine. Each SCU, for example Turbines, is a business in its own right, operating as an internal supplier to CFBUs. SCUs undertake the detailed design of modules and components, which are integrated into a whole product by CFBU systems designers.

3. Research and Technology (R\&T): R\&T is responsible for developing technological capabilities that can be used in the design and manufacture of products by SCUs and CFBUs. The primary purpose of R\&T is to ensure new technologies are mature enough to be used within in-service products. As discussed previously, safety is a critical feature of product designs, which means that technologies have to be thoroughly tested and verified before being used on an in-service design.

Taken together, these three areas of design responsibility define the strategic design context, summarised in Figure 2.14.

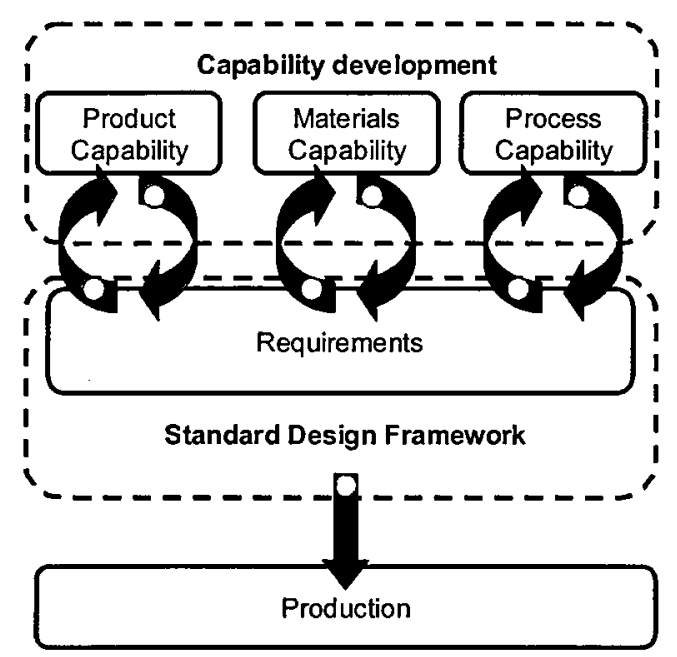

Figure 2.14: Strategic design context

The standard design framework in Figure 2.14 encompasses systems and tactical design activities within CFBUs and SCUs. When suitably mature, product, material and manufacturing capabilities developed within R\&T are available for use within the standard design framework. Activities to develop technological capabilities would be classed as design in the broadest sense of the term. This could be interpreted to mean that a significant amount of design work has already happened before design activities take place within the standard design framework. Both capability development and the standard design framework operate through a series of governance processes, which 
are used to run programmes, reflecting the organisational philosophy shown in the previous section.

Capability development and the standard design framework form distinct parts of the overall set of activities used to design products within Rolls-Royce. Investigating how environmental considerations could be integrated into design decisions, it is necessary to clarify the 'design' activities within Rolls-Royce that are relevant to the scope of this research. This research will focus on integrating environmental considerations into the standard design framework only, for the following reasons:

1. It is being hosted within an engineering design department within a CFBU, with the mandate to investigate how environmental considerations could be integrated into design decisions specifically from this design perspective.

2. The nature of the design for environment capability being developed. From the introduction in chapter 1 , the capability describes creating environmental impact profiles by picking from menus of materials and processes. If this were the case, these must be suitably mature to be used within an inservice design. R\&T effectively creates capabilities to be placed on these menus within the standard design framework.

The next section provides more detail on the structure of the standard design framework.

\subsubsection{Standard design framework}

This section provides a summary of the governance process used to operate the standard design framework. The standard design framework is based on the principle that the 'design process is how a product definition is derived from a set of requirements' (O'Toole, 2009, p.5). This principle defines the two sides of the design and definition ' $V$ ', shown in Figure 2.15. Designs are created by capturing requirements on the one side and creating definitions to meet these requirements on the other. Requirements come from the customer, which are translated into technical product requirements and flowed into engineering. Figure 2.15 also defines the different levels of design within the standard design framework, working down from a product level, to sub-system and component design. As discussed previously, whole engine integration (systems design) is the responsibility of CFBUs. Detailed subsystem and component definitions are generally owned by SCUs. 


\section{Verification}

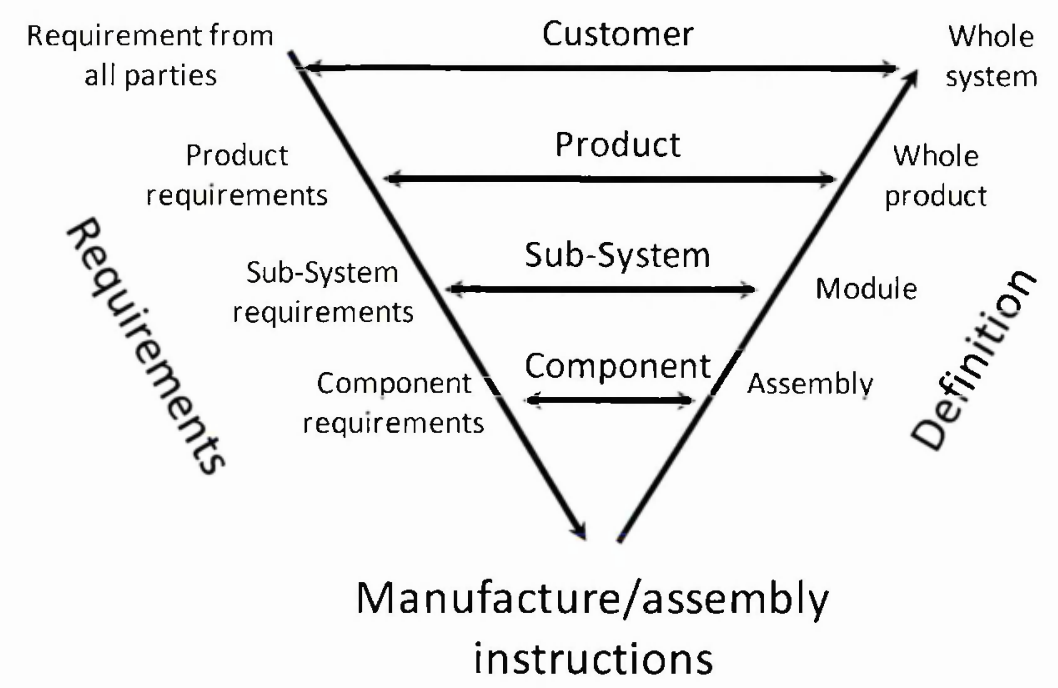

Figure 2.15: Simplified Rolls-Royce engineering process (O'Toole, 2009)

At each level of design the principle of flowing down requirements and integrating product definitions from a component level upwards is applied. The design process is intended to be fractal in this way, applying the same processes and structure irrespective of the specific design level and context. This also includes the application of a gated process to manage the progression from requirements capture through to when a product is ready to be released into service.

Product Introduction and Lifecycle Management (PILM) is the gated process used to operate programmes, summarised in Figure 2.16. Design governance processes are formed beneath PILM, applied from a product down to a component level.

\begin{tabular}{|c|c|c|c|c|c|}
\hline $\begin{array}{c}\text { Stage 0 } \\
\text { Innovation \& } \\
\text { Opportunity } \\
\text { Selection }\end{array}$ & $\begin{array}{c}\text { Stage 1 } \\
\text { Preliminary } \\
\text { Concept } \\
\text { Definition }\end{array}$ & $\begin{array}{c}\text { Stage 2 } \\
\text { Full } \\
\text { Concept } \\
\text { Definition }\end{array}$ & $\begin{array}{c}\text { Stage 3 } \\
\text { Product } \\
\text { Realisation }\end{array}$ & $\begin{array}{c}\text { Stage 4 } \\
\text { Production \& } \\
\text { In-Service } \\
\text { Support }\end{array}$ & $\begin{array}{c}\text { Stage 5 } \\
\text { Continuing } \\
\text { In-Service } \\
\text { Support }\end{array}$ \\
\hline
\end{tabular}

Figure 2.16: Rolls-Royce PILM process (O'Toole, 2009)

Although PILM is the overall process that controls design activities, it is actually a risk management process (Rolls-Royce, 2012). PILM has been designed to ensure that all technical, commercial and regulatory requirements have been satisfied for a product before it enters into service. Requirements are set at each stage of the process. Compliance is checked through review meetings that invite design teams to answer standard sets of questions asked by specialist reviewers. A design is only allowed to progress to the next stage if the relevant questions are satisfactorily answered. The purpose of each of the PILM stages is as follows: 
- Stage 0: Identify business opportunities and select those which satisfy the defined business and technical requirements. This ensures a sufficiently robust business case exists before significant resources are committed to product development;

- Stage 1: Develop and flow down requirements and develop product concepts that satisfy these requirements. At stage 1 exit a concept is downselected for detailed design. A product level general arrangement drawing is typically produced at this stage;

- Stage 2: First review of the whole detailed product design, conducted through the Preliminary Design Review (PDR). Component definitions are typically set at this stage, including material selection and manufacturing considerations;

- Stage 3: Refinement of the product design leading up to the final review of the whole detailed product design, conducted through the Critical Design Review (CDR). Parts are released for manufacture and assembly begins to commence leading in to product test (validation). Once testing is completed (this can take up to 18 months for a large civil aerospace engine) the product enters into service;

- Stage 4: Controls requirements for the in-service support of products that are currently being manufactured and are in-service with existing customers;

- Stage 5: Controls the on-going support for products that are no longer being sold as new although are continuing in-service;

- Stage 6: Bringing products out of service, including disposal considerations.

Design governance processes are covered within the first three stages of PILM. These governance processes operate a similar gated approach built around the design stages summarised above:

- Define requirements;

- Develop and select concepts;

- Preliminary design, including the PDR;

- Detailed design, including the CDR;

- Validate design.

Figure 2.17 provides a detailed breakdown of Design Review (DR) gates that structure the design governance process, also indicating how these align with PILM stages one to three. 


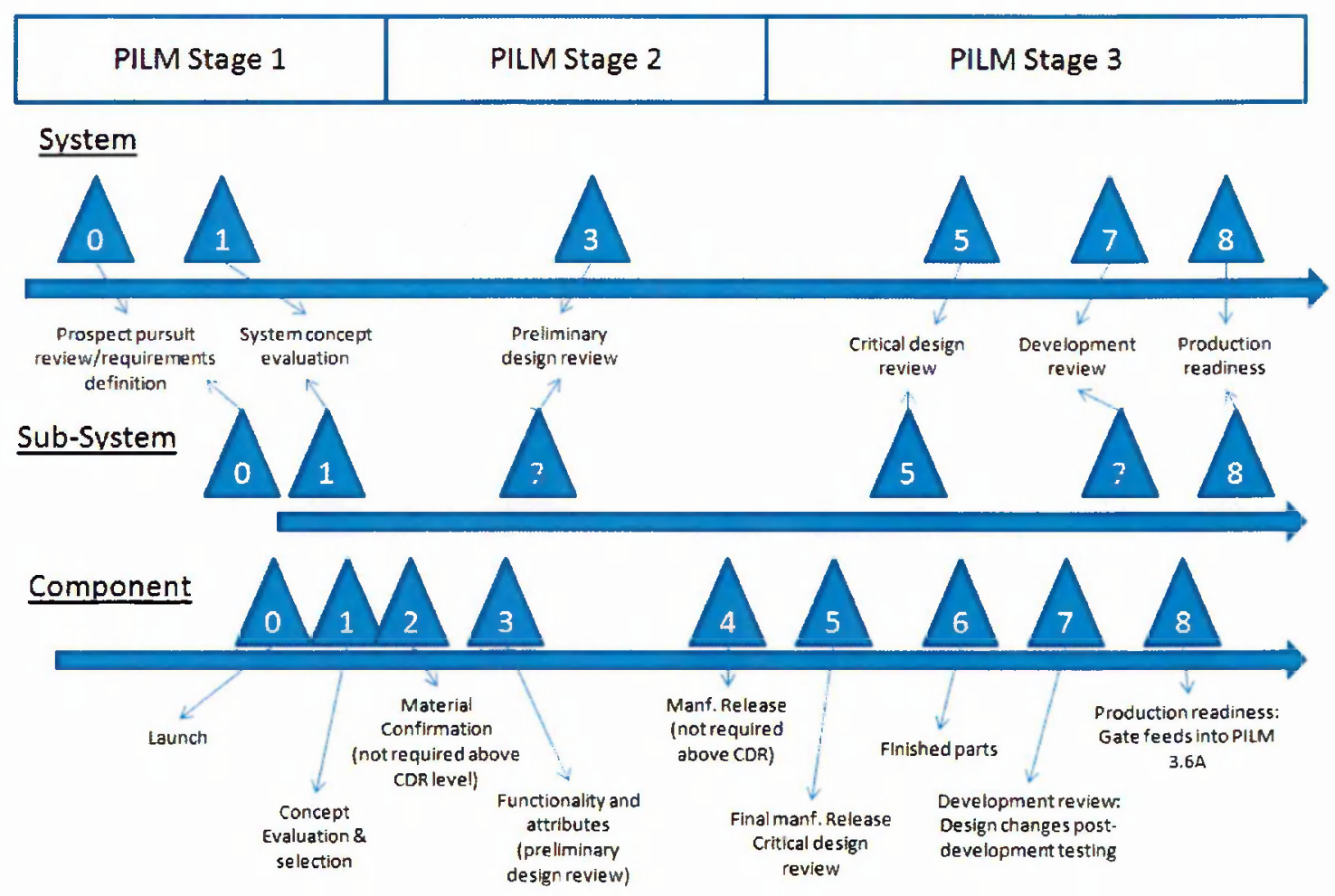

Figure 2.17: Design Review (DR) gates summary

From a component level upwards, the DR gates are broadly structured as follows:

- DRO: Understand and define requirements;

- DR1: Generate concepts and concept selection;

- DR2: Confirm material selection and manufacturing route (only applies at a component design level);

- DR3: Preliminary design review. This is the first review of the detailed design definition;

- DR4: Initial parts released to manufacturing (only applies at a component design (evel);

- DR5: Critical design review. At this point the design essentially becomes fixed. All components are released to manufacturing;

- DR6: Finished parts are received (only applies at a component design level);

- DR7: Reviews the outcomes of the development programme (product testing);

- DR8: Release into production.

The gated review process described here provides the overarching framework that forms the engineering design governance process. Several things are required for the process to operate in reality: 
- Defined process sub-steps, providing more detail on what is expected at each stage;

- Design tools, used to support the completion of design activities at each stage. Tools also provide evidence to reviewers that design definitions meet requirements;

- Sets of review questions that are used to check a design has reached a suitable level of maturity before each review gate;

- Review meetings, which are usually chaired by a Chief Design Engineer and used to make a decision on whether or not the design can progress to the next stage of the process.

The same design process is applied to all design problems at all design levels, whether performing a re-design task that involves modifying a single dimension on an old product or defining a whole new product. Questions posed at each review gate are tailored from a standard list to suit the particular design situation.

It is possible to draw a distinction between two parts of the standard design framework. The first part, operating at the front end of the framework, deals with strategic issues that are concerned with understanding customer requirements and how, at a product level, these can be fulfilled with the supply of products and services. The second part begins once a product concept has been selected and requirements are flowed down into engineering where the detail of the design is defined. Given this distinction, and relating this discussion back to the research theme of integrating environmental considerations into design decisions, it is necessary to provide further definition on what is within the scope of this research. This research will focus on integrating environmental considerations into detailed design decisions only, for the following reasons:

1. As described in the introduction, at a strategic level environmental problems are already well considered as part of standard design decision making processes. For civil aerospace products in particular, environmental requirements are the second most important consideration after safety. However, within this context only use-phase environmental impacts are considered. Where the company struggles is addressing environmental impacts from the other phases of the life cycle within design, related to material production, manufacturing and end-of-life. Logically this places a focus on tactical design as this is where material and manufacturing choices, which influence these parts of the life cycle, are mainly considered.

2. The nature of the design for environment capability being developed. As mentioned previously, the capability described in chapter 1 focuses on 
creating environmental impact profiles by picking from menus of materials and processes. This also suggests a focus on tactical design problems, rather than strategic issues related to how environmental considerations influence what products are being made.

Within the standard design framework a suite of design tools is used to support the completion of the various design tasks. Given the goal of attempting to integrate environmental considerations into existing processes and ways of working (as concluded in section 2.3.1), a brief summary of relevant tools should provide insights into how technical information is currently used in design decisions in Rolls-Royce. Existing tools may also be suitable for considering environmental information as well as information related to other technical disciplines. A brief summary of tools is provided in the next section.

\subsubsection{Tools used within the design process}

There are variety of tools used within the Rolls-Royce standard design framework to help with the completion of various design tasks, from generating concepts to validating detailed designs. Amongst others, these include (from O'Toole, 2009):

- Systems engineering, including requirements management and functional analysis techniques;

- Robust design, including design of experiments;

- Quality Function Deployment (QFD);

- Brainstorming and mind-mapping;

- Risk management, including Design Failure Modes and Effects Analysis (DFMEA);

- Design for manufacture.

Typical design tools such as Computer Aided Design (CAD) and Finite Element Analysis (FEA) packages are also used.

It is not practical to provide an in-depth review of all of these tools. However, more detail on those that could be relevant to integrating environmental considerations into design decisions is appropriate. Design experts within Rolls-Royce suggested looking at systems engineering and risk management for considering environmental issues in design. Systems engineering was recommended as it promotes a holistic approach to understanding the full design context and it was acknowledged that considering the environment at a detailed design level is one area that is currently overlooked. Risk management, including DFMEA, was highlighted as important, as managing risk is 
used to drive design activity. Risk could also be used to allow for environmental issues to be considered alongside other design requirements.

From O'Toole (2009), systems engineering is an interdisciplinary approach for creating successful systems, through focusing on:

- Defining needs, requirements and functionality early in the design process;

- Applying systems thinking to understand hierarchies of systems, subsystems and inter-relationships;

- Developing an understanding of the full design context, for example: operations, cost and schedule, manufacturing, test, support and disposal;

- Developing 'parts of a system not a system of parts', whilst considering that 'one person's system is another person's sub-system' (O'Toole, 2009, p.18);

- Programme leadership of technical programmes.

Through its focus on systems thinking, systems engineering has had a strong influence on the structure of the overall design process, in particular the design-definition ' $V$ ', as shown in Figure 2.15. Many tools are related to systems engineering, for example requirements management, functional analyses and context diagrams. Context diagrams are an important tool in understanding the broader design problem and may be suitable for prompting the consideration of environmental issues.

Risk is defined as an uncertain event with the ability to 'impact on the achievement of objectives', measured by the probability of the event occurring and the impact it would have on objectives if it did (O'Toole, 2009). It is acknowledged that all designs carry risk (as defined) and that this should be reviewed periodically throughout the design activity. To manage risk, a cyclical process is applied as shown in Figure 2.18. Brainstorming, workshops and interviews are used to identify events that can impact on objectives. These are then assessed using measures of probability and impact to determine significant risks, which require treatment actions.

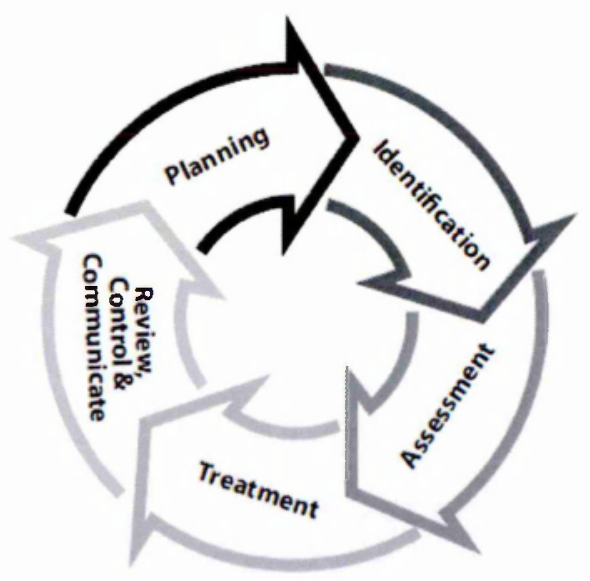

Figure 2.18: Risk management cycle (O'Toole, 2009) 
Alongside standard risk management activities, Design Failure Modes and Effects Analysis (DFMEA) is a process that is applied to focus specifically on technical risks and how these can be mitigated through design activity. Technical risk focuses on the engineering aspects of a design, seeking to broadly identify how a solution can fail to fulfil requirements. Figure 2.19 provides an overview of the DFMEA process.

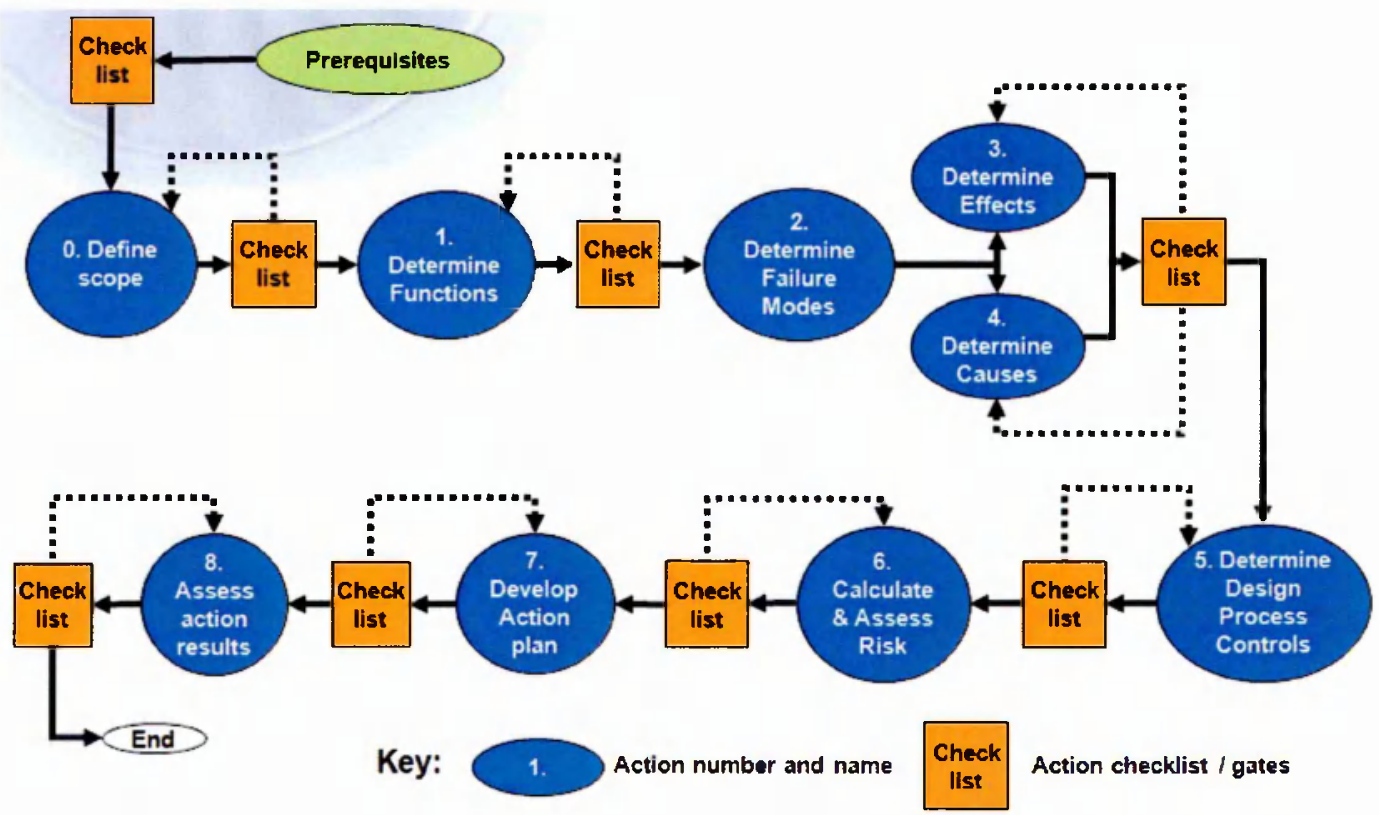

Figure 2.19: Rolls-Royce DFMEA process (Lloyd, 2012b)

DFMEA is structured the same as a normal risk assessment, although with some important differences:

- Systems engineering techniques are used to bound the scope and functions of the assessment around a particular design problem;

- Risks are structured by recording a failure mode (how a design can fail to fulfil its functions or non-functional requirements), cause (physical mechanism that leads to failure) and effect (what the customer might ultimately experience);

- Risks are assessed by not only scoring the probability and impact (linked to the cause and effect), but also detectability. Detectability is an assessment of the assurance that design activities will prevent risks becoming a reality;

- This leads to actions that can be taken in design to mitigate risks.

DFMEA is applied to drive design actions by identifying significant risks and how they can be mitigated in design, highlighting how risk is an important part of design at RollsRoyce. 


\subsubsection{Summary of the context and conclusions}

The purpose of this section was to provide an understanding of the business and design context, as an integral part of understanding how environmental considerations could be integrated into design decisions in Rolls-Royce. A brief overview of literature on the topic of organisational culture and structure was used to develop an interpretation of the organisational culture and structure within Rolls-Royce. It was found that, whilst being structurally task orientated, there is also a strong role culture within Rolls-Royce. This role culture is reflected in the existence of central corporate functions, with 'daughter' functions at a CFBU level, which are responsible for controlling how all aspects of the business' programmes are executed. The existence of a strong role culture within Rolls-Royce emphasised the importance of developing an approach to integrating environmental considerations in a way that was consistent with existing ways of working, which was a conclusion also taken from the DfE literature reviewed in section 2.2 .

Section 2.3.2 provided an overview of the Rolls-Royce design process, to investigate the existing design decision context within which environmental considerations were to be integrated. Building on observations of how the overall Rolls-Royce business structure influences the structure of design processes, the strategic design context was set by defining design activities that take place within capability development and those that take place within the standard design framework. Focusing on the standard design framework, the Rolls-Royce design philosophy was defined as how product definitions are derived from a set of requirements' (O'Toole, 2009). Being strongly influenced by systems engineering, the design philosophy is embodied by the design definition ' $V$ ' (Figure 2.15), which defines the different design levels from a whole system down to detailed component design. At each level the same gated process is applied, which is formed within an overall risk management process (PILM). Tools are applied within the standard design framework to aid the completion of the various design tasks. Systems engineering and risk management were briefly reviewed, being suggested as relevant to integrating environmental considerations into design decisions. Risk was discussed as being a particularly important part of the design process, not only forming the design process at a high level but also being used to drive design actions at a detailed level through technical risk management processes.

Several relevant findings can be used to guide research direction:

- Through gaining an understanding of the organisational philosophy within Rolls-Royce, the importance of developing an approach to integrating environmental considerations into design decisions that is consistent with 
current ways of working and making design decisions has again been highlighted.

- Reviewing the design context, it has been concluded that this research should focus on integrating environmental considerations into design decisions at a detailed design level within the standard design framework. This is due to the part of the organisation within which the researcher was situated, the nature of the design for environment capability being developed (as described in chapter 1) and the fact that at a strategic level environmental issues already have a strong influence on design decisions (as also discussed in chapter 1 ).

- Risk management forms an important part of design within Rolls-Royce. A gated review process to manage risks forms the overarching design process and risk management is also used to drive design activities at a detailed design level.

The review of design processes has helped to define the scope for this research. Relating the defined design scope to the levels of DfE discussed in section 2.2.2.1, focusing on integrating environmental considerations at a detailed design level within the standard design framework is consistent with a level 1 product improvement approach to DfE. At this level the goal is to incrementally improve the environmental performance of products through minor modifications using tactical design strategies. Developing new technologies through R\&T would be consistent with level 2 ecoinnovation improvements.

\subsection{Informal interviews}

This section briefly summarises the findings from informal interviews held with various environmental specialists, design managers and experts from outside Rolls-Royce during the first year of the research. Interviews were applied informally as a research technique, reflecting their use as a common tool for preliminary research to gain an understanding of the problem to guide research direction. As noted by Fielding and Thomas (2001), when exploring problems the most instinctive approach is to ask questions'. The following points summarise the main findings from these interviews that were used to guide research direction:

- To integrate environmental considerations into design decisions environmental information needs to be presented in a way that can be compared and traded with other design requirements. 
- The approach needs to be integrative, working within existing processes and tools.

- It is necessary to overcome what some environmental specialists refer to as the 'so what' factor. In the past environmental information has been provided to designers, although how this information is relevant to their design tasks has not been communicated clearly. This has led to designers finding the environmental information interesting, although concluding 'so what'?

- There are perceptions that due to the product use phase being responsible for the vast majority of environmental impacts over the life cycle of an aero engine, non-use phase environmental impacts are insignificant and can be overlooked. Any approach needs to overcome this perception by highlighting the importance of addressing non-use phase impacts within design.

- Clear environmental priorities are needed.

- Environmental information needs to quickly point to some form of tangible action.

\subsection{Summary and conclusions}

The aim of this chapter was to investigate how environmental considerations could be integrated into design decisions within Rolls-Royce. An overview of the topic of DfE concluded that developing a thorough understanding of the business and design context was important. It was also highlighted how successful approaches to DfE developed methodologies to support the use of DfE tools and were also based on the setting of environmental priorities.

Following this conclusion, an overview of the Rolls-Royce business context and design processes has been provided, setting the scope of this research at tactical design level. The importance of developing an approach that is consistent with existing ways of working was highlighted, as well as emphasising the importance of risk within the design process. Findings from informal interviews conducted with experts within and outside of the company emphasised the need to present environmental information in a way that could facilitate design trade-offs, highlight the importance of addressing nonuse phase environmental impacts and overcome the 'so what' factor. 
Concluding this research theme, based on the evidence presented this research will utilise risk to integrate environmental considerations into design decisions in RollsRoyce. A risk based approach meets several important requirements:

- Using risk will present environmental impacts in a format that can be related to standard design requirements and included within design trade-offs.

- Such an approach would be consistent with existing processes and ways of working.

- If environmental impacts can be translated into risks it will highlight why the problems are important to address, overcoming current perceptions that non-use phase environmental impacts are insignificant.

- A risk approach would lead into activities that will develop mitigating actions, pointing to what designers can do to address environmental impacts.

The next chapter begins a new research theme focusing on how risk can be used to integrate environmental considerations into design decisions in Rolls-Royce. 


\section{Developing the framework}

This chapter presents the research activities undertaken to investigate how risk could be used to integrate environmental considerations into design decisions within RollsRoyce, leading to the development of the framework for environmental risk management. Section 3.1 presents the research methods applied for this part of the research. Section 3.2 reviews how risk is managed generally within Rolls-Royce. Based on the findings from this review, section 3.3 explores more broadly what is meant by 'risk' to define a perspective on risk for this research, including the terminology to be used. Based on the defined risk perspective, section 3.4 builds the framework for environmental risk management for using risk to integrate environmental considerations into design decisions. Section 3.5 provides a summary and conclusions for this chapter leading into chapter 4 , which begins to test the framework by identifying environmental business hazards.

\subsection{Methodology}

This part of the research represents a new research theme and action research cycle, following on from the research presented in chapter 2 (Figure 3.1). As in chapter 2, research was taking place within the real-world context of an organisational setting, with the aim of producing outcomes that could lead to change within that organisation. Research approaches within this iteration of the action research cycle again needed to be exploratory, understanding the problem situation in order to guide the development of new research themes. Literature reviews and informal interviews were applied as tactics for exploration. Details of how these approaches were applied, and for what purpose, are described throughout this chapter. 


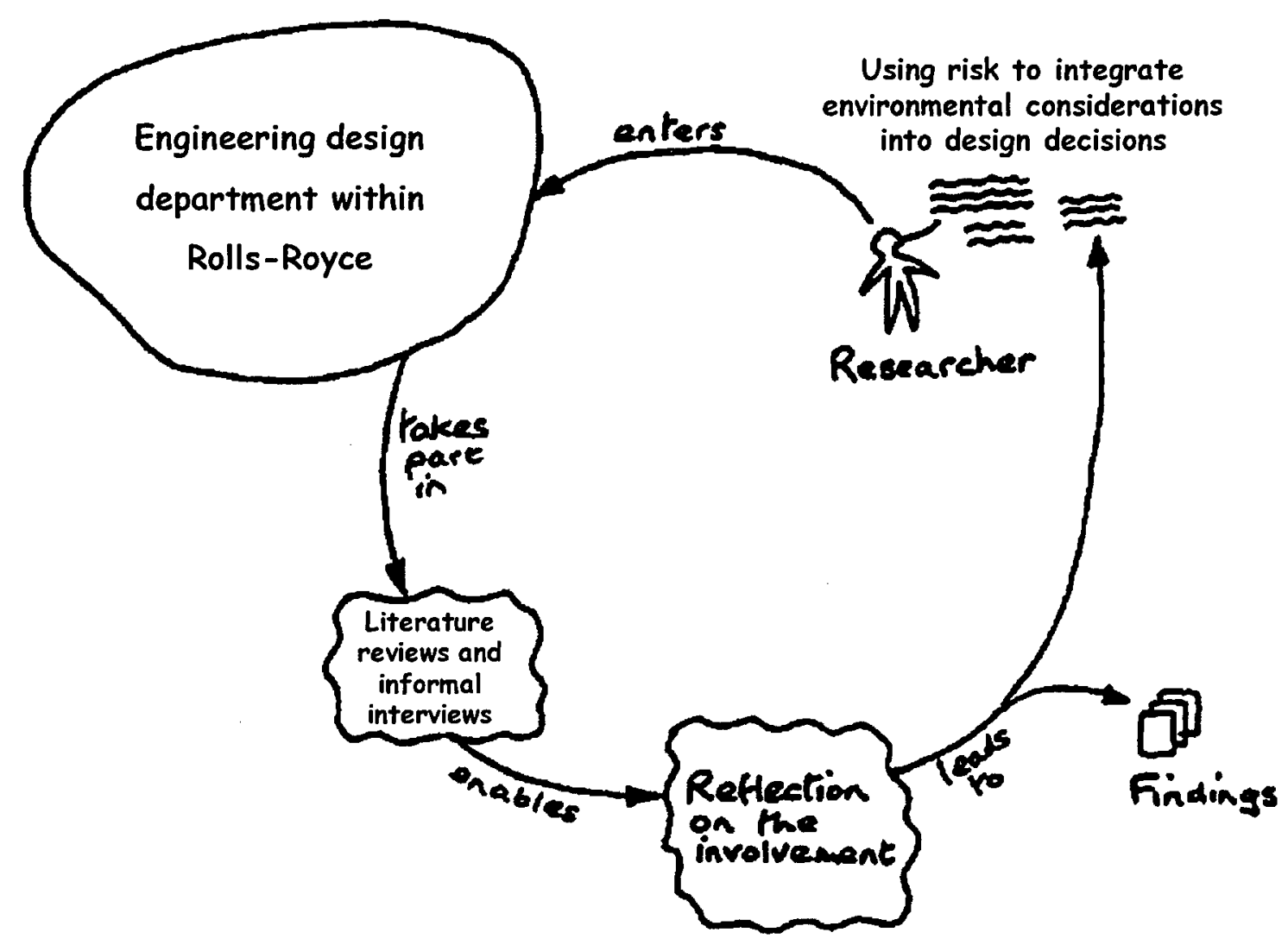

Figure 3.1: Action research cycle for investigating using risk to integrate environmental considerations into design decisions (adapted from Checkland and Holwell, 1998)

\subsection{Risk management within Rolls-Royce}

This section explores how risk is managed generally within Rolls-Royce, as an obvious first step in understanding how risk could be used to integrate environmental considerations into design decisions. Informal interviews were also conducted with risk managers, focusing on the topic of how risks posed by environmental issues are currently managed by the business and how environmental considerations needed to be integrated into design decisions.

This section begins by reviewing the risk management system and framework applied within Rolls-Royce in section 3.3.1. Section 3.3.2 links the review of risk management systems with the brief overview of risk management in design provided in chapter 2 . Section 3.3.3 briefly summarises findings from informal interviews with risk managers within Rolls-Royce, leading to a summary and conclusions and a more general investigation into the concept of risk in section 3.3. 


\subsubsection{Risk management structure and process}

From its 2012 annual report (Rolls-Royce, 2012), risk management within Rolls-Royce forms part of its corporate governance. Risk management is seen as a key part of Rolls-Royce fulfilling its business objectives and risks are defined as threats to the achievement of business objectives or the continuing reputation of the group' (RollsRoyce, 2012, p.51). Figure 3.2 provides an overview of the Rolls-Royce risk management structure, which is based around how the business is planned. Risk management systems are applied to the creation of the group strategy, down through the various business units, programmes and projects.

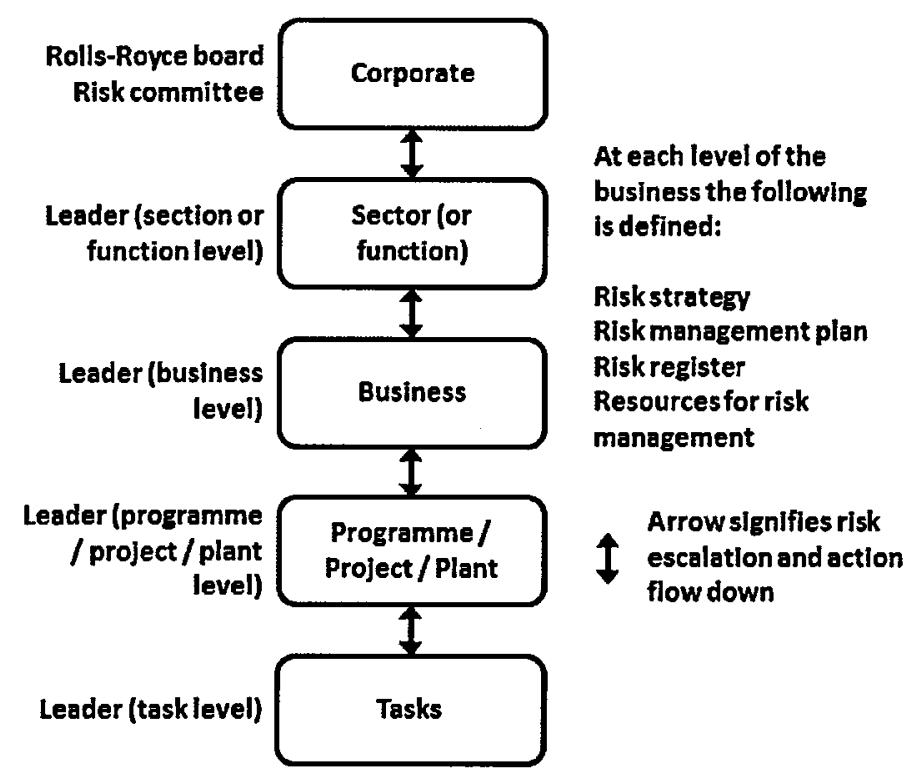

Figure 3.2: Risk management structure (adapted from Rolls-Royce, 2009)

A risk strategy and management plan is defined at each level of the business where objectives are set. Risk Management Plans (RMPs) provide the context for risk management activities at any level of the business, defining:

- Roles and responsibilities of those tasked with managing risks, usually led by a senior executive;

- The scope of the business activity and objectives against which risk can be assessed;

- Risk assessment criteria, which define how the impact and probability of risks are to be measured;

- How risks are to be escalated within the business;

- A risk register to record and track risks as they are identified, assessed, and if necessary, treated.

The scope of the activity and business objectives against which risk is assessed are set from a corporate level downwards, as indicated in Figure 3.2. At a corporate level, 
Rolls-Royce's primary objective against which risks are assessed is the Ten Year Forecast (TYF), which calculates expected profits, by business unit, from projected product sales and contract margins (that is the expected revenue from a contract minus the expected cost of delivery, discounted for net present value). Within businesses, at a project or programme level, objectives are laid out in work breakdown structures, which outline the various tasks that need to be completed in order to fulfil objectives. Objectives can be commercial or technical and are generally linked to requirements documents that detail the various aspects of a project (commercial, services, product etc.) Typical objectives at a programme level include:

- Time and budget (commercial);

- Weight, unit cost and specific fuel consumption (sfc) (technical);

- Maintenance arisings (services).

Table 3.1 shows a typical set of risk criteria taken from the RMP of a large civil aerospace engineering programme within Rolls-Royce, illustrating the diversity of objectives against which risk is assessed. Criteria are set and agreed at the beginning of the programme relevant to its objectives.

Table 3.1: Example risk criteria (Preston, 2010)

\begin{tabular}{|c|c|c|c|c|c|c|c|}
\hline \multirow{2}{*}{ Category } & \multirow{2}{*}{ Probability } & \multicolumn{5}{|c|}{ Impact } & \multirow[b]{2}{*}{ Noise } \\
\hline & & Time & ENPV $^{*}$ & Unit cost & Weight & $s f c^{\star \star *}$ & \\
\hline Very Low & $\leq 1 \%$ & $<1$ week & $<£ 0.5 m$ & $<£ 3 k$ & $<12$ lbs & $<0.03 \%$ & $<0.06 \mathrm{~dB}$ \\
\hline Low & $>1 \%$ to $\leq 5 \%$ & $\begin{array}{c}1 \text { to } 2 \\
\text { week slip } \\
\text { to plan }\end{array}$ & $\begin{aligned} \geq & £ 0.5 \mathrm{~m} \text { to } \\
& <£ 2 \mathrm{~m}\end{aligned}$ & $\begin{array}{l}\geq £ 3 k \text { to } \\
<£ 5 k\end{array}$ & $\begin{array}{l}12 \text { to } 20 \\
\text { Ibs }\end{array}$ & $\begin{array}{l}0.03 \text { to } \\
0.05 \%\end{array}$ & $\begin{array}{l}0.06 \text { to } \\
0.1 \mathrm{~dB}\end{array}$ \\
\hline Medium & $>5 \%$ to $\leq 25 \%$ & $\begin{array}{c}2 \text { to } 9 \\
\text { week slip } \\
\text { to plan }\end{array}$ & $\begin{array}{c}\geq £ 3.5 m \text { to } \\
<£ 17.5 m\end{array}$ & $\begin{array}{l}\geq £ 5 k \text { to } \\
<£ 25 k\end{array}$ & $\begin{array}{l}20 \text { to } \\
100 \mathrm{lbs}\end{array}$ & $\begin{array}{l}0.05 \text { to } \\
0.25 \%\end{array}$ & $\begin{array}{l}0.1 \text { to } \\
0.5 \mathrm{~dB}\end{array}$ \\
\hline High & $\begin{array}{l}>25 \% \text { to } \\
\leq 50 \%\end{array}$ & $\begin{array}{c}9 \text { to } 18 \\
\text { week slip } \\
\text { to plan }\end{array}$ & $\begin{aligned} \geq & £ 17.5 \mathrm{~m} \text { to } \\
& <£ 35 \mathrm{~m}\end{aligned}$ & $\begin{array}{l}\geq £ 25 \mathrm{k} \text { to } \\
<£ 50 \mathrm{k}\end{array}$ & $\begin{array}{l}100 \text { to } \\
200 \mathrm{lbs}\end{array}$ & $\begin{array}{l}0.25 \text { to } \\
0.5 \%\end{array}$ & $\begin{array}{l}0.5 \text { to } 1 \\
\mathrm{~dB}\end{array}$ \\
\hline Very High & $>50 \%$ & $\begin{array}{l}>18 \text { week } \\
\text { slip to } \\
\text { plan }\end{array}$ & $\geq £ 35 \mathrm{~m}$ & $\geq 50 \mathrm{k}$ & $>200 \mathrm{lbs}$ & $>0.5 \%$ & $>1 \mathrm{~dB}$ \\
\hline
\end{tabular}

*Values expressed in Net Present Value (NPV) are discounted at 10\% p.a. over the ten year forecast ${ }^{* *}$ specific fuel consumption

Risks are managed through the levels of the business using the risk escalation process, which is indicated by the arrows between business levels shown in Figure 3.2. Escalation is controlled according to the magnitude of the risk and risk criteria set at each level of the business. Escalation is typically based on financial criteria only as this is a common objective against which risk is assessed at all levels of the business. For example, corporately the business may not be concerned with risks to a programme meeting a technical objective. However, not meeting that objective could have financial implications warranting consideration at a board level. 
A standard process to manage risk (Figure 3.3) is applied wherever organisational objectives are set within the business structure described.

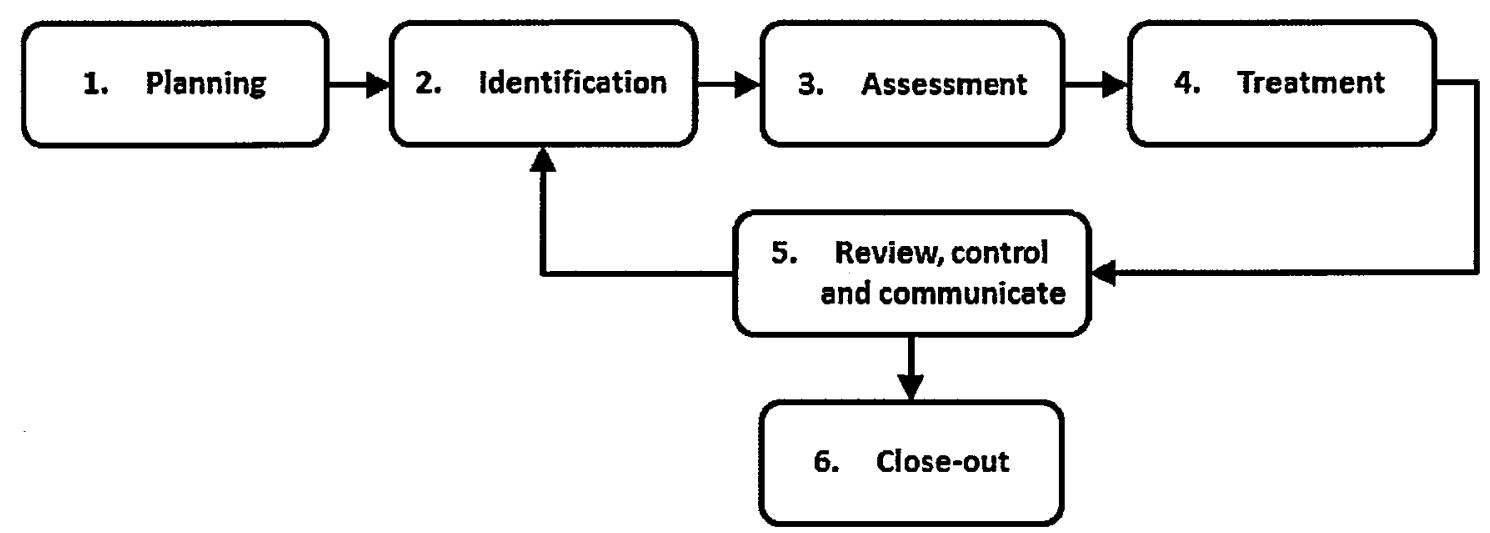

Figure 3.3: Risk management process (adapted from Rolls-Royce, 2009)

From Rolls-Royce (2007b), initial planning activities should be completed as part of defining the RMP for a given activity (as listed previously). Risk identification is the next step, the purpose of which is to record events that may impact on the objectives of the activity. Identified risks are structured in an 'if...then' format, capturing the root cause (if) and impact (then) of an event. Several techniques are used to identify risks, the most popular being brainstorming workshops and interviews. Prompt lists and sources of information (for example previous risk assessments) can be used to help identify risks. Emphasis is placed on ensuring the right stakeholders are involved in the identification of risks, for example those with specialist knowledge or experience.

From Rolls-Royce (2007b), risk assessment involves assessing the probability and potential impact of a risk and calculating the product of the two to evaluate significance, which will determine whether any treatment actions are required. Probability and impact can be assessed qualitatively or quantitatively. In certain cases a qualitative assessment may identify the need for quantification. Table 3.2 provides examples of typical qualitative risk assessment criteria. 
Table 3.2: Qualitative risk assessment criteria (Ro/ls-Royce, 2007b)

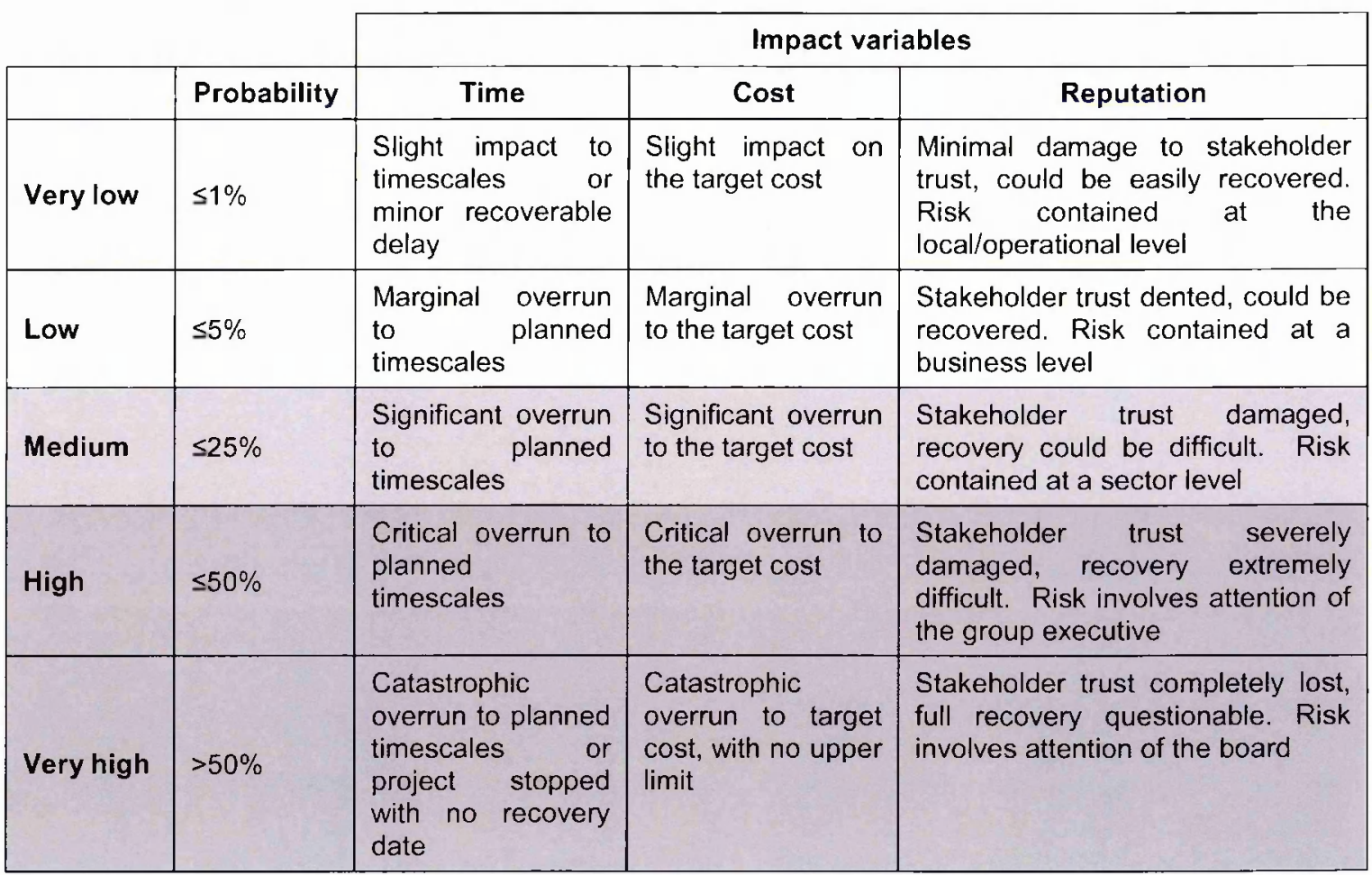

It is typical to estimate numerical impact values for all risks, derived from estimations, previous knowledge or expert judgement. Quantitative assessments may be necessary where a greater accuracy or confidence in the estimates of probability and impact are required. These can only be calculated where numerical values exist, typically using statistical analysis based on historical data. In the end, assessments of probability and impact inevitably depend on the nature of the risk (Rolls-Royce, 2007b).

Probability Impact Diagrams (PIDs) are used to assess risk, an example of which is shown in Figure 3.4.

\begin{tabular}{|c|c|c|c|c|c|c|}
\hline \multirow{5}{*}{$\begin{array}{l}\text { 름 } \\
\frac{0}{0} \\
\frac{0}{0} \\
\frac{0}{2} \\
0\end{array}$} & $\geq 50 \%$ & 9 & 14 & 19 & 24 & 29 \\
\hline & $225 \%$ to $<50 \%$ & 7 & 12 & 17 & 22 & 27 \\
\hline & $25 \%$ to $<25 \%$ & 5 & 10 & 15 & 20 & 25 \\
\hline & $\geq 1 \%$ to $<5 \%$ & 3 & 8 & 13 & 18 & 23 \\
\hline & \multirow[t]{3}{*}{$\ll 1 \%$} & 1 & 6 & 11 & 16 & 21 \\
\hline & & Very Low & Low & Medium & High & Very High \\
\hline & & \multicolumn{5}{|c|}{ Impact } \\
\hline
\end{tabular}

Figure 3.4: Example Probability Impact Diagram (PID) (Rolls-Royce, 2007b)

The use of a PID requires assessments of probability and impact to be translated into a common scale. By plotting each on the axis of the diagram the product of probability 
and impact can be calculated to provide an assessment of risk. Scores within the diagram in Figure 3.4 indicate how risks with a very high impact are deemed to be more severe (Rolls-Royce, 2007b).

PIDs can also be used to define boundaries of the acceptability of risks, as shown by the shaded areas in Figure 3.4. These boundaries are used to determine whether actions are required to treat a risk. A risk treatment hierarchy is broken down as follows (from Snape, 2009):

- Dark shaded area: signifies 'key risks' requiring treatment actions;

- Grey shaded area: signifies 'significant risks', which should be treated if a cost effective or common strategy exists;

- White area: signifies 'underlying risks', which should be monitored, although no risk treatment is required.

From Rolls-Royce (2007b), the purpose of risk treatment is to reduce the probability or impact of risks to an acceptable level. Treatment strategies generally fall into one of the following four categories (Rolls-Royce, 2007b):

- Mitigate: specifying actions that will reduce the probability and/or impact of the risk to an acceptable level;

- Transfer: where risks raised fall outside the scope of the risk assessment, but can be transferred to another activity;

- Accept: in the case where nothing more can reasonably be done to mitigate a risk;

- Reject: if no other treatment strategy is feasible, which essentially means that the original plan within which the risk resides is rejected. This strategy requires a new plan.

Risk treatment should move risks from the top right to the bottom left of the PID (Figure 3.5).

\begin{tabular}{|c|c|c|c|c|c|c|}
\hline \multirow{5}{*}{ 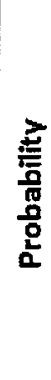 } & $\geq 50 \%$ & 9 & 14 & 19 & 24 & 29 \\
\hline & $\geq 25 \%$ to $<50 \%$ & 7 & 12 & & 22 & 27 \\
\hline & $\geq 5 \%$ to $<25 \%$ & 5 & 10 & & & 25 \\
\hline & $\geq 1 \%$ to $<5 \%$ & 3 & 8 & 13 & 18 & 23 \\
\hline & \multirow[t]{3}{*}{$<1 \%$} & 1 & 6 & 11 & 16 & 21 \\
\hline & & Very Low & Low & Mediun & High & VeryHigh \\
\hline & & \multicolumn{5}{|c|}{ Impact } \\
\hline
\end{tabular}

Figure 3.5: Effects of risk treatment (Rolls-Royce, 2007b) 
Risk treatment is the final step in the risk management framework. As indicated in Figure 3.3, risk management is intended to be a continuous exercise. To meet this aim regular risk reviews are conducted as part of risk management activities. Risk reviews assess the effectiveness of mitigating actions implemented to reduce significant risks to an acceptable level and also allow for the identification of new risks.

\subsubsection{Engineering and risk management}

Chapter 2 provided a brief overview of risk management as it is applied during design activities. Further discussions with designers and risk managers within Rolls-Royce have shown the two distinct risk management activities within engineering design discussed in chapter 2 :

- Application of the standard risk management framework (as described in section 3.2.1) to manage project and programme risks;

- Applying DFMEA to manage technical risks, focused on how a design solution can fail to meet requirements and what design activities can be taken to treat these potential failures (see section 2.3.2.3).

From the designers' perspective, technical risk management was seen as distinct from 'non-engineering' risk management. Risk managers noted the opposite perspective, focusing on risk management as a project activity, overlooking considerations for technical risk management activities within engineering. Both groups interviewed noted the importance of considering how engineering failures can have a subsequent impact on business performance. Engineering has been described as a risk management activity in itself (Glazier, 2009) and it is important to consider how these activities form part of a business' overall approach to managing risk. Those interviewed agreed that, whilst standard risk management and technical risk management activities are seen as distinct, an important outcome of engineering risk management is to form an input in to project and programme risk management.

\subsubsection{Informal interviews}

As well as reviewing internal literature on risk management, informal interviews with risk managers were also used to gain an understanding of how Rolls-Royce manages risk. Interviews were applied informally as a research technique, similar to the approach described in chapter 2, reflecting their use as a common tool for preliminary research to gain an understanding of the problem to guide research direction. 
The topics of interview discussions included risk management within Rolls-Royce in general, although, in keeping with the research theme for this chapter, there was a specific focus on any relationships between risk management and the management of environmental issues by the business. Of particular interest was gaining insights into how risk could be used to integrate environmental considerations into design decisions. The following points summarise the main findings from interview discussions, which were used to guide research direction:

- It was acknowledged that environmental issues pose a risk to the business. One risk manager highlighted how climate change was listed on the corporate risk register, leading to strategic design activities to improve the efficiency of the products made by Rolls-Royce.

- The corporate environmental department should be responsible for the identification of environmental issues that pose a risk to the business and determining how these need to be managed. It was also suggested that a risk register specifically for environmental issues could be created.

- One risk manager noted that, due to the complex nature of environmental problems, business risks posed by these issues might be difficult to assess.

- Risks posed by environmental issues should be managed like any other risk within the standard risk management structure and framework within RollsRoyce. Adding appropriate prompts to risk identification lists was suggested as one way of ensuring risks from environmental issues are considered.

Discussions with the Head of Enterprise Risk Management highlighted how risk was a very broad concept. It was recommended to investigate the topic of risk more thoroughly to understand precisely the types of risks this research sought to manage. Following this recommendation, the next section reviews academic literature on the general topic of risk to define the meaning of risk for this research, and the terminology to be used.

\subsection{Defining risk for this research}

The meaning of the term risk can be confusing, as it differs between a multitude of different perspectives (RCEP, 1998; Undrill, 2007). Risk terminology is also applied interchangeably between different risk perspectives to mean different things, which creates difficulties in understanding (Gerrard and Petts, 1998). Despite there being no agreed universal definition of risk (Aven and Renn, 2009; Aven and Renn, 2010; Rosa, 2010), there is agreement that any definition does have the following common properties (from Adams, 1995; Aven and Renn, 2010; Renn, 1998b): 
1. A distinction between reality and possibility;

2. An interest in both desirable and undesirable outcomes (possibilities);

3. Consideration for uncertainty not just probability, which is a narrower concept applied as a means of measuring uncertainty;

4. A broad scope without restrictions to specific consequences or quantities.

The first property is obvious, as 'if the future were predetermined...the term 'risk' would make no sense' (Renn, 1998b, p.50). Properties two and three can be combined to view risk as a 'two-dimensional concept covering the severity of events and consequences and uncertainties about the occurrence of the events and consequences' (Aven and Renn, 2010, p.256). The final property reflects the fact that 'risk comes in many forms' (Adams, 1995, p.21), encompassing many different types of outcomes and sources of uncertainty.

Given the many perspectives on risk and confusions over terminology, for clarity, it is necessary to define what is meant by risk for this research and the terms to be used. The following sections provide a brief overview of risk as it applied within the contexts of business, engineering and the environment, similar to the three technical perspectives on risk from Renn (1998b). The purpose of this review is to not to provide an in depth analysis of each perspective on risk. Instead, the goal is to use the information reviewed to define the different components of risk and highlight some of the differences in terminologies. Findings from this review can then used to develop a perspective on risk for this research and define the terms to be used here.

\subsubsection{Risk in a business context}

In a business context risk is defined as the 'effect of uncertainty on objectives' (BSI, $2009, \mathrm{p} .1$ ), which is 'often expressed in terms of a combination of the consequences of an event and the associated likelihood' (BSI, 2009, p.1). Risk management is defined as 'coordinated activities to direct and control an organisation with regard to risk' (BSI, 2009, p.2). Business risk management has developed relatively recently, partly in response to financial scandals in the 1990s (Power, 2009). Following these scandals a series of recommendation reports for improvements in corporate governance were published, notably the 'Turnbull report' (ICAEW, 1999), which made recommendations for improvements in accounting internal controls. Importantly, the report also mentions the need for organisations to manage risk more broadly, including those issues related to non-financial matters (ICAEW, 1999). Controversies such as the Brent Spar also highlighted the need for risk management beyond financial concerns (Löfstedt and 
Renn, 1997), reflected in a wider growing need for organisations to act in a responsible and socially acceptable manner (Power, 2004).

Guidance for managing risk in organisations is provided in an international standard, ISO 31000 (see BSI, 2009), which is reviewed in the following pages. Leitch (2010) criticises this standard for its ambiguous style and some of this ambiguity will have transferred into the following review. Despite these criticisms the standard has been widely adopted (Purdy, 2010), so it was judged to be a valid reference for providing an overview of risk management in a business context.

ISO 31000 introduces a framework for developing risk management within an organisation, which is shown in Figure 3.6.

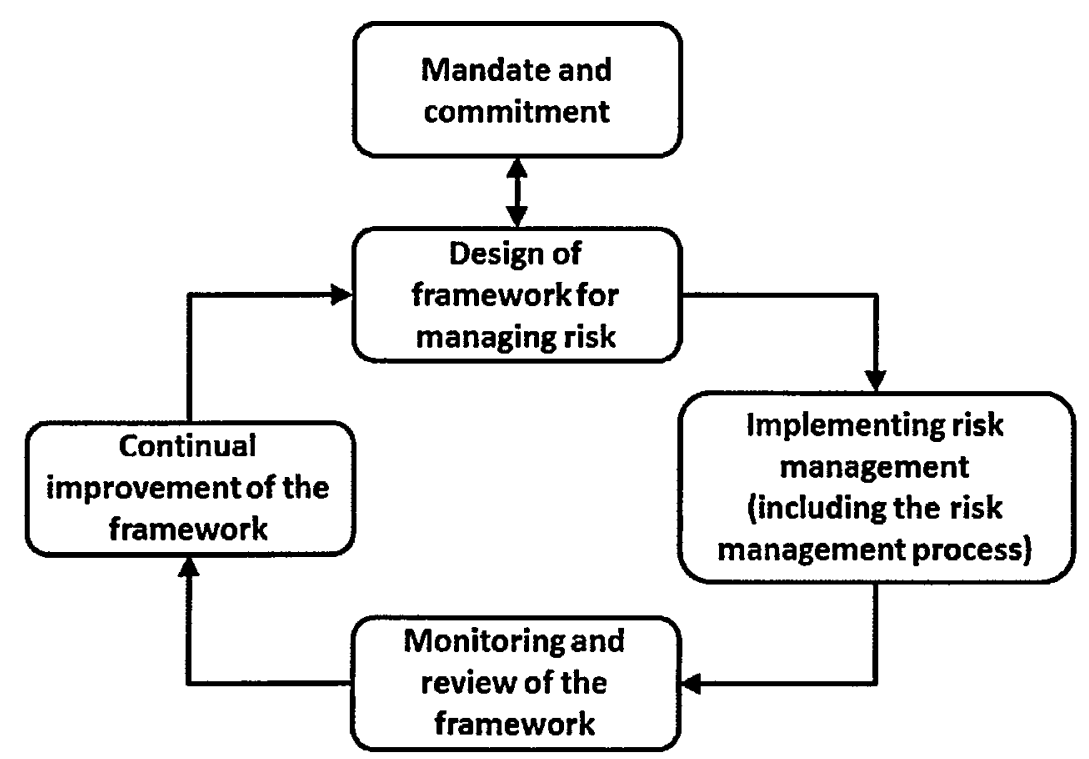

Figure 3.6: Framework for managing risk (adapted from BSI, 2009)

Emphasised at the top of the framework, 'mandate and commitment' is the first component, which states that effective risk management requires strong leadership from senior management (BSI, 2009). Other components of the framework form a 'plan-do-check-act' cycle for implementing risk management into an organisation (Pojasek, 2011, p.90). Designing a framework for managing risk is the first component within this cycle. The framework should be developed to suit an organisation through an evaluation of the organisational context, including its processes for internal governance, organisational structure, business objectives, decision making processes and culture (Pojasek, 2011; Purdy, 2010). Through developing the framework the following should also be defined (from BSI, 2009, pp.10-12):

- A risk management policy that aligns risk management objectives with the objectives of the organisation;

- Organisational accountabilities for risk management; 
- How risk management is integrated into organisational processes through the use of risk management plans;

- Resources for risk management;

- Internal and external mechanisms for the communication of risks.

Elements of this framework are similar to the Rolls-Royce risk management structure, described in section 3.2.1 and Figure 3.2.

One of the main purposes of the framework is to implement risk management through deployment of the risk management process (Purdy, 2010), which is described in section 3.3.1.1. Monitor and review and continual improvement are the components that complete the framework cycle. Monitoring is required to ensure the risk management framework remains effective, which can be evaluated by (from BSI, 2009, p.13):

- Developing and periodically reviewing risk management performance indicators;

- Reviewing any deviations from risk management plans;

- Re-evaluating an organisation's external and internal contexts to ensure the design of the risk management framework is still appropriate.

Continual improvement is focused on using results of monitoring and review to identify actions that can be implemented to improve the framework and risk management within the organisation (BSI, 2009).

\subsubsection{Risk management process}

Figure 3.7 shows the risk management process, to be applied to manage risk at all levels of an organisation where objectives are defined (BSI, 2009). 


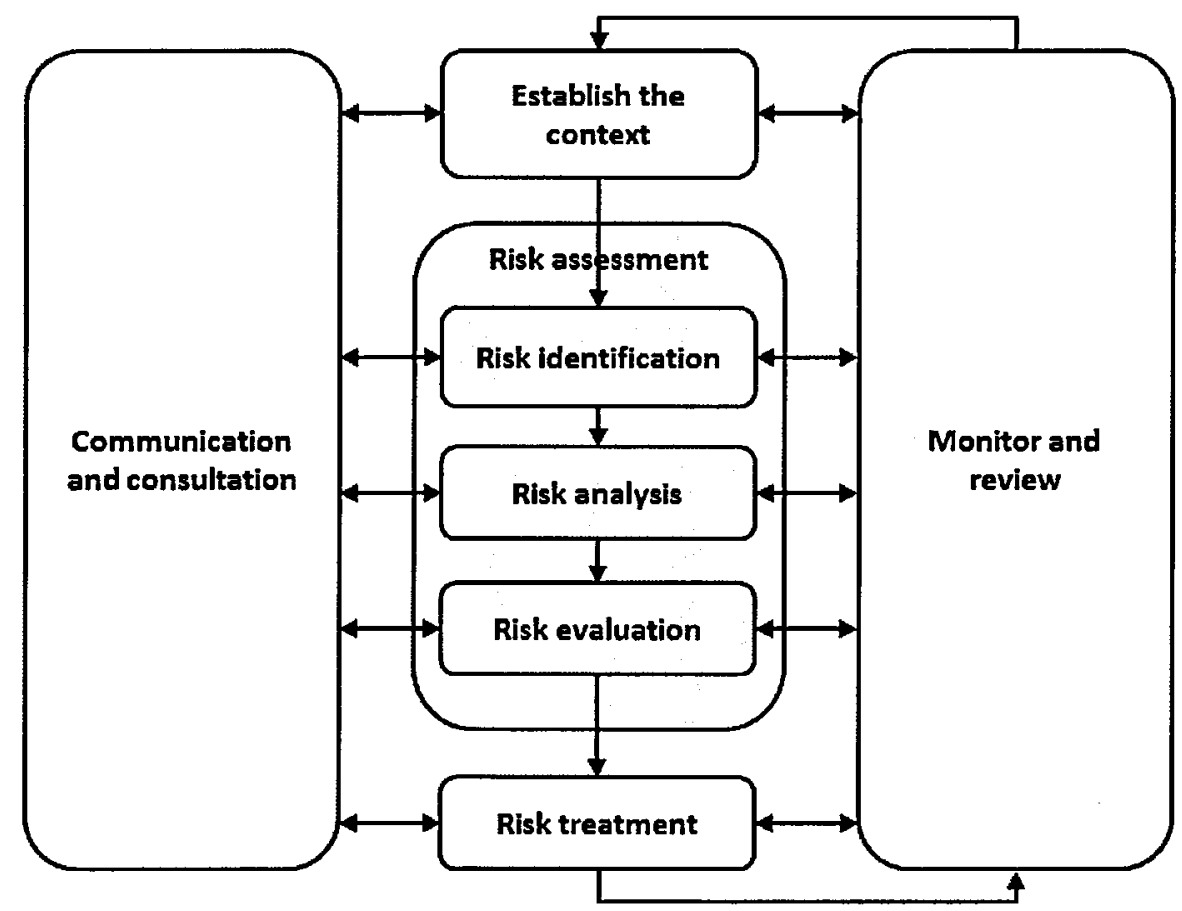

Figure 3.7: Risk management process (adapted from BSI, 2009)

Establishing the context is the first step in the process, the purpose of which is to define the activities that are to be subject to risk assessment, what the objectives of these activities are and to identify the internal and external factors that may impact on the successful achievement of these objectives (Purdy, 2010). Establishing the context should also define the risk criteria, including (from BSI, 2010, p.10):

- The types of consequences to be included in a risk assessment and how these will be measured;

- How probabilities will be expressed;

- How a level of risk will be determined (although not articulated, it is assumed this should be based on the measurements of probabilities and consequences defined);

- Criteria defining when a risk will need to be treated.

From Figure 3.7, risk assessment covers the steps of risk identification, risk analysis and risk evaluation. Risk identification involves the application of a systematic process to record events or situations that can impact upon objectives (BSI, 2010). Methods for identifying risks include (from BSI, 2010, p.12):

- Evidence based methods, such as checklists or past risk assessments;

- Systematic team approaches applying prompts or questions (further clarification on what these approaches might be is not provided);

- Brainstorming;

- Inductive reasoning, for example using hazard and operability studies (HAZOP, described in section 3.3.2). 
Risk analysis focuses on understanding the consequences of each risk and the likelihood of these consequences. Guidance in the standard does not stipulate how measures of the consequences and likelihoods should be combined to measure risk. Purdy (2010) summarises the advice as follows:

'The way in which consequences and likelihood are expressed and the way in which they are combined to determine a level of risk should reflect the type of risk, the information available, and the purpose for which the risk assessment output is to be used. These should all be consistent with the risk criteria.' (Purdy, 2010, p.884)

Whatever approach is deemed to be appropriate, qualitative, semi-quantitative or quantitative methods can be used to analyse risks (BSI, 2010). The guidance highlights the value of using expert judgements in situations where a quantitative analysis is not possible or sensible (BSI, 2010).

From BSI (2010), risk evaluation is concerned with determining the significance of risks and whether actions to treat risks are required, determined using risk criteria set at the beginning of the risk management process. Other considerations should also be factored into risk treatment decisions, for example ethical, financial and legal issues, including any potential differences in how risks might be perceived. Whether a risk should be treated can also be decided by balancing the costs and benefits of risk treatment. Common practice suggests dividing risks into three bands (from BSI, 2010, p.16):

1. Intolerable risks, which should be treated irrespective of the cost;

2. Medium risks, which could be treated depending on a balance of the costs and benefits of doing so;

3. Negligible risks, where no risk treatment is required.

From BSI (2009), treatment is the final step of the process, which should apply actions to modify intolerable or medium risks to reduce the risk to an acceptable level, as defined by the risk criteria (BSI, 2009). Results of treatment actions should be reviewed to assess the residual risk (defined as the risk remaining after risk treatment) and whether or not further treatment actions are required. Risk treatment actions should be implemented through risk treatment plans.

Communication and consultation, and monitor and review, are 'continually applied elements of the process' (Purdy, 2010, p.883), as illustrated by the position of these elements in Figure 3.7. Communication and consultation highlights the need to engage with internal and external stakeholders through all stages of the risk management 
process (BSI, 2009). Consulting stakeholders can be particularly important for (from BSI, 2009, p.14):

- Establishing the context appropriately;

- Bringing together different areas of expertise to assess risks;

- Ensuring different views are considered when establishing risk criteria and evaluating risks, in particular external stakeholders who may perceive risks differently;

- Securing agreement for risk treatment plans.

The purpose of monitoring and review is to introduce an iterative element into the risk management process, ensuring appropriate actions are taken to identify new risks and monitor how existing risks change over time (Purdy, 2010). This can involve detecting changes in the internal and external contexts, improving risk assessment through the identification of better information and applying lessons learned through the application of the risk management process (BSI, 2009).

\subsubsection{Summary}

This section has reviewed risk management in a business context, with a particular focus on ISO 31000 , which contains internationally agreed guidance on how to manage risk within organisations. Authors of the standard acknowledge that there are many different interpretations of risk and risk management processes, explicitly seeking to 'achieve consistency and reliability in risk management' by publishing the standard (Purdy, 2010, p.881). It is also acknowledged that this creates challenges for those who use different language and approaches, encouraging 'compromise and change' to achieve their aim (Purdy, 2010, p.881). Taking the standard as the default interpretations for risk management in a business context, the following terms are defined to summarise this review:

- Risk: effect of uncertainty on objectives, which, when not taken literally, is interpreted to mean the potential effect of events which are currently uncertain on the extent to which objectives are achieved' (Leitch, 2010, p.889);

- Risk management: the overall set of activities and processes for managing risks within an organisation;

- Risk assessment: elements of the risk management process including identifying, analysing and evaluating risks;

- Risk analysis: measuring risk by understanding and combining the consequences and likelihood of a risk. 


\subsubsection{Risk in an engineering context}

The Royal Academy of Engineering published a series of reports with a specific focus on risk, including reports covering common methodologies for risk assessment and management (RAEng, 2003a) and the societal aspects of risk (RAEng, 2003b). RAEng (2003a) appears to be an engineer's view on the business risk management literature reviewed in the previous section. Support is explicitly given to the influence of the Turnbull report (ICAEW, 1999) and its recommendations on corporate risk. One differentiating factor between the methodologies report from the Royal Academy of Engineering and the business management literature is that the engineer's view specifically sets risk management within the context of the engineering life cycle, which can broadly be said to be the same as the life cycle of a project (and should not be confused with the product life cycle as applied in LCA, described in chapter 2). As also discussed in chapter 2, a similar approach is applied at Rolls-Royce through the PILM (Product Introduction and Life Cycle Management) process.

The strategic nature of the Royal Academy's methodologies report (RAEng, 2003a) appears to be intentional. However, there is a broader body of engineering risk management literature that focuses on the risks that can come from failure, related to the operation of products and processes. Of interest is what the consequences of these failures might be, with an emphasis on effects to business performance, human health, the environment, or all three (Kletz, 1999, p.80).

In the engineering context a distinction is sometimes made between hazard and risk; hazard being defined as 'a property or situation that in particular circumstances could lead to harm' and risk as 'a combination of the probability, or frequency, of occurrence of a defined hazard and the magnitude of the consequences of the occurrence' (RCEP, 1998, p.51). However, other engineering perspectives do not make this distinction, following conventions for the use of the term risk within a business context. This point is illustrated by an alternative definition of risk from the engineering literature as the probability that an untoward event will happen, multiplied by the impact it could have if it did happen' (RAEng, 2003b, p.4).

Conflicts exist between differing terminologies, which makes the precise nature of the activities described by terms such as risk analysis, risk assessment and risk management within an engineering context difficult to pin down. From the literature reviewed, it is the researcher's interpretation that the process of understanding risk within engineering typically involves the following steps (from Kletz, 1999; Pitblado and Turney, 1996; Pleyss, 1995; RAEng, 2003a; RCEP, 1998; Renn, 1998a; Stirling, 1998): 
- Identify hazards: identifying events, situations or properties that could lead to harm;

- Risk assessment (sometimes called risk or hazard analysis): ‘a scientific process for defining risk in precise, often quantitative terms', which involves specifying undesirable consequences resulting from the occurrence of a hazard and calculating the probability of these consequences occurring, aggregating the two (Renn, 1998a, p.52);

- Risk management: Undertaking activities to reduce risks to a tolerable level, including defining what that level is.

An important first step for understanding risk in an engineering context is precisely defining the scope of a risk assessment. Systems engineering approaches (as described in chapter 2) can be used to define the physical aspects of a product, process or plant that are to be analysed, how these physical aspects interact with other physical components, people or the environment, and how these interactions can lead to risks (RAEng, 2003a). Part of this scoping exercise should also focus on determining what consequences are to be included within an assessment and how these should be measured (Fischhoff et al., 1984).

Prominent tools and techniques that are used to understand risk include (from Kletz, 1999; Pitblado and Turney, 1996):

- Hazard and operability studies (HAZOP) and hazard analysis (HAZAN), the latter also being referred to as Quantitative/Probabilistic Risk Assessment (Q/PRA) (Kletz, 1999, p. 78);

- Failure Modes Effect (and Criticality) Analysis (FMEA/FMECA);

- Event and Fault Tree Analysis (ETA/FTA);

- Checklists.

Techniques vary between adopting a top down deductive (i.e. starting with a failure and thinking about how this could occur), bottom up inductive (i.e. thinking about events and how these can lead to failure) or combination of approaches to identify hazards and assess risks. The following sections provide a brief overview of each, categorised according to those that can be used for identifying hazards and those used for assessing risk.

\subsubsection{Identifying hazards}

HAZOP is a structured technique for identifying hazards, predominantly applied within the chemical process, oil and gas and nuclear industries, and should ideally be 
conducted during plant design (Kletz, 1999). HAZOPs identify hazards through the use of guide words to generate potential deviations from the 'designer's intention' (Pitblado and Turney, 1996, p.14). These deviations can be reviewed to determine possible causes, consequences and whether or not remediating actions are required (Kletz, 1999). For example, relating to the flow through a pipe between two parts of a plant (from Kletz, 1999), guide words could include, NONE, MORE OF, LESS OF, AS WELL AS (this list is not exhaustive). Taking NONE as an example, meaning no flow through the pipe, the following questions can be asked:

- Is no flow possible?

- If so, how?

- What would happen if there was no flow? How would this affect the operation of the plant?

- Should no flow be prevented?

- Do the potential consequences justify modifying the pipe design to prevent no flow?

HAZOPs use a combination of deductive and inductive reasoning, using a deductive approach to identify deviations and an inductive logic to determine what the effects of deviations might be (Dunjó et al., 2010). The intention is for HAZOPs to be conducted by a team of people, with the aim of fostering a creative approach that will encourage new ways of looking at problems (Kletz, 1999). Such an approach makes conducting HAZOPs quite subjective (Dunjó et al., 2010) and the quality of the study can be dependent on the experience of the people involved. Dunjó et al. (2010) view the subjectivity of HAZOPs as one of the strengths of the technique. Another strength is its inherent simplicity (Pitblado and Turney, 1996).

An alternative approach to identifying hazards is FMEA, an inductive method more commonly used when studying mechanical equipment (Pitblado and Turney, 1996). From Healey et al. (1994), FMEA is based on analysing all conceivable ways in which a part or component can fail and what the effects of this might be. Failures are usually considered in isolation and can be analysed in terms of either physical structure or function.

ETA and FTA are techniques usually used within QRA (Quantitative Risk Assessment), although when applied qualitatively they can be used as methods for identifying hazards (Pitblado and Turney, 1996). Both approaches are based on the principle of developing graphical models of how failures can occur either deductively (FTA) or inductively (ETA). Graphical representations are usually in the form of logic diagrams, 
defining the sequential relationships between initiating events and their consequences, or vice versa.

\subsubsection{Risk assessment}

Within the process industries in particular, HAZAN (short for hazard analysis) is a term used to describe techniques for assessing risks quantitatively in terms of their potential consequences and probability of occurring. According to Kletz (1999), HAZAN also includes the practice of determining a 'target criterion' against which the probability of consequences (the risk) can be evaluated. Pitblado \& Turney (1996) use different terminology, defining the process for assessing the consequences and probabilities of a hazard as QRA, and referring to the evaluation of risks as the application of risk assessment'. Whatever terms are used, the process of analysing hazards is concerned with answering three questions (from Kletz, 1999, p.83):

1. How often?

2. How big?

3. So what?

From Pitblado and Turney (1996), the first question is related to 'event probability estimation', which can be based on the use of historical statistical data or a failure logic method (ETA or FTA), applying probability estimates to each contributory factor.

The second question seeks to determine the magnitude of the consequences (Pitblado and Turney, 1996). From Pitblado and Turney (1996), significant bodies of research have been conducted to determine what are called effect and vulnerability models. Effect models are concerned with attempting to determine the exact physical nature of the event, for example the size of an explosion or toxic release. Vulnerability models are used to determine the damage caused to people, facilities and the environment. Example assessments could include potential structural damage given a size of explosion, or attempts to model the effects of a toxic release using dose response relationships (this is similar to environmental risk assessment discussed in the next section).

FMEA can be combined with a criticality analysis (CA) to analyse risks identified using this technique. The aim of the CA is to assess the severity and likelihood of the occurrences identified by the FMEA, which can be done either qualitatively using judgement, or quantitatively when data is available. Estimates of the severity (consequences) and likelihood can be combined and the failure modes ranked to allow an assessment of significance. 


\subsubsection{Risk management}

Risk management is concerned with answering the final question listed above, attempting to deal with the problem of assessing the significance of risks and determining what actions are required to reduce risk to an acceptable level (Renn, 1998a). Assessing significance can be a contentious process, as it inevitably involves value judgements around defining what level of risk is 'tolerable' (Stirling, 1998).

During the 1990s there was debate on whether judgements of tolerability should rely purely on objective technical risk assessments and the extent to which subjective values and perceptions also merited consideration within decision making processes, in particular those from non-experts and the public (Renn, 1998a; Stirling, 1998). Prevailing conclusions appear to acknowledge how technical assessments are inevitably influenced by social dimensions, including the assumptions that frame an assessment (Stirling, 1998), differences in how risks are perceived (RAEng, 2003b) and how risks need to be communicated (Fischhoff, 1995). An outcome of this has been the acknowledgment of the importance of involving broader groups of stakeholders within risk assessment and management (RAEng, 2003b). Whilst not doubting the value of detailed technical analysis, acknowledging how risk assessments are framed by social dimensions should help improve the perceived validity of the results of technical risk assessments (RAEng, 2003b; Stirling, 1998).

Strategies to manage risks deemed to be unacceptable can include (from RAEng, 2003a, p.13):

- Avoid: make fundamental changes so the risk is no longer an issue. This strategy aligns with 'reject' from the Rolls-Royce risk treatment strategies listed in section 3.2.1 (p.60);

- Reduce: by either altering the chances that an untoward event will happen, or the consequences that will be incurred if it did. This aligns with mitigate from the Rolls-Royce strategies. It could also align with 'transfer' if the risk reduction is achieved through in-service monitoring, for example;

- Accept: that the risk may be realised and that nothing can be done to reduce it.

\subsubsection{Summary}

This section has given a brief summary of risk within the engineering context, providing brief insights into the tools and techniques that can be used for identifying and assessing risks and how these need to be managed. Figure 3.8 provides a summary 
risk framework, outlining the steps described to understand risk in an engineering context.

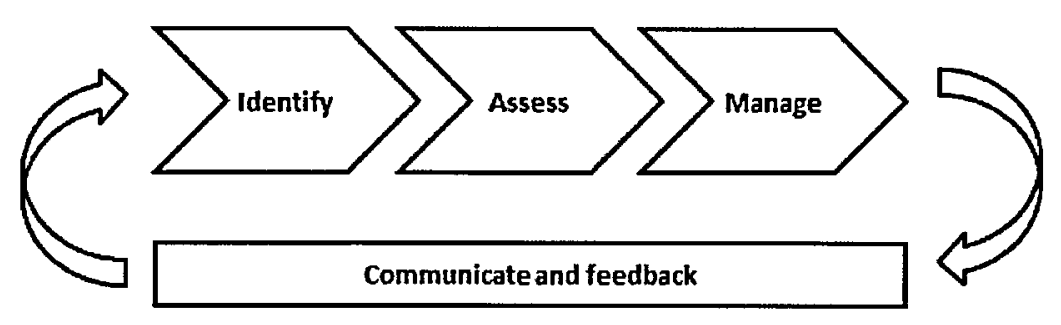

Figure 3.8: Summary risk framework (RAEng, 2003a)

\subsubsection{Risk in an environmental context}

Risk as applied within an environmental context is derived from the engineering context (RCEP, 1998), although typically maintains a distinction between hazard and risk; definitions of hazard and risk from $\operatorname{DETR}(2000, p .11)$ are identical to those provided by the Royal Commission on Environmental Pollution (RCEP, 1998, p.51). However, whereas risk in an engineering context can also include an analysis of effects on business performance, risk in an environmental context focuses solely on undesirable effects on the environment and human health (Cowell et al., 2002). Commonly referred to as Environmental Risk Assessment (ERA) (Pollard et al., 2002), the field has developed largely from the need to take a more proactive approach to environmental damage (DETR, 2000) and is indicative of developments in environmental policy that seek to take a precautionary approach (RCEP, 1998).

A similar framework for risk assessment to the one outlined in Figure 3.8 is applied within ERA, in that it broadly follows a process of identifying hazards, assessing risks and determining how they need to be managed. However, ERA applies different approaches to identify and assess risks within this framework. Similar to the application of systems engineering to define the scope of a risk assessment within engineering, ERA develops conceptual models to define the scope of an assessment, from which four components of a risk are defined (from DETR, 2000):

- Source (S): of hazards;

- Pathway $(P)$ : means by which a hazard comes into contact with a receptor;

- Receptor (R): target of interest in terms of assessing the effects of a hazard;

- Impact (I): effect on the receptor resulting from exposure to the hazard.

From DETR (2000), together these form the SPRI relationships linking the sources of hazards with effects on the environment and human health. Conceptual models are used to identify hazards, creating visual and written representations of possible SPRI 
relationships. Identified hazards can then be assessed in terms of their consequences and likelihood, which can be combined to give an overall appreciation of the risk. Initially this can be done qualitatively using risk screening to prioritise risks that may require quantitative assessment.

From RCEP (1998), techniques reviewed in section 3.3.2 can be used to quantitatively assess the probability that a hazard might occur. However, estimating the magnitude and probability of the consequences from that hazard once it has occurred can be more complicated. The source of this complexity comes from uncertainties in establishing cause-effect relationships between a particular hazard and subsequent effects on the environment. For example, regarding the effects of chemicals in the environment, determining the effect requires knowledge of the toxicity of substances, what effects they can have on humans and the environment, at what doses, and what pathways and exposures potentially exist. The critical factor is determining the predicted exposure to a substance and comparing this to the level of exposure at which adverse effects might occur (Graedel and Allenby, 1995).

Once a risk has been assessed the final step is to evaluate the risk in terms of its significance to determine how it should be managed (DETR, 2000). Environmental assessments can involve a greater degree of uncertainty, which can make the evaluation process more challenging than in other contexts (Graedel and Allenby, 1995), in particular as value judgements play an important role and risk perceptions are often affected by cultural views (Adams, 1995). In the past there has been a similar debate in ERA to the one in engineering risk management, regarding whether scientific 'objective' risk assessments should be kept 'separate from the social and political aspects of decision making' (Gerrard and Petts, 1998, p.2). Gerrard and Petts (1998), RCEP (1998) and Stirling (1998) all argue for the integration of the social and technical dimensions of risk, for the same reasons as those articulated in section 3.3.2.3, which appears to be the prevailing consensus.

\subsubsection{Risk and LCA}

Chapter 2 provided an overview of LCA as a means of assessing the environmental impacts of a product, within the context of its use as a DfE tool. Given the relevance of LCA and risk to this research, it was interesting to investigate any links between these approaches.

There are clear differences between the concepts of risk assessment and LCA as they have been described. However, considerations have been made for how the two tools 
can be used together. Owens (1997) provides a critique of LCA and in particular LCIA methods and their perceived limitations. Owens suggests that LCAs should be used as a 'screening and identification process' to 'pose questions for risk assessment...to resolve' (Owens, 1997, p.364). Discussions with an LCA specialist led to a similar conclusion, highlighting how risk assessment is used as a more robust approach for assessing ecotoxicity impacts in particular, overcoming perceived limitations in standard LCIA assessment methods.

Sharratt and Choong (2002) provide an alternative perspective, which suggests LCA can be used as an input into assessing business risks posed by environmental impacts within process industry projects. Through their PERA (Process Environmental Risk Assessment) methodology, Sharratt and Choong (2002) outline how business risks can be identified and assessed through identifying stakeholders, being defined as 'an individual or a group who has an interest in the company because s/he can affect, or is affected by its activities' (Sharratt and Choong, 2002, p.481). Suggested stakeholders include customers, governments, pressure groups, shareholders and the financial services sector, acknowledging how the business risk 'arises not directly as a result of an event or situation, but to the actions of the company's stakeholders in response to that event' (Sharratt and Choong, 2002, p.480). It is suggested that environmental impacts can pose a business risk by leading to stakeholder responses such as changes in operating costs from environmentally related regulation, difficulties of waste disposal, loss of licences to operate and subsequent effects on business image (amongst others). In order to execute PERA the environmental impacts relevant to plant need to be known, for which the use of LCA is suggested.

\subsubsection{Summary}

This section has provided a brief overview of risk in the environmental context, which is derived from the engineering context although with a specific focus on risks posed to the environment and human health. Figure 3.9 provides a summary framework for risk in the environmental context, which deliberately sets the scientific process of risk assessment within a broader risk management decision making process, including the need to consider how risks are perceived and communicated. 


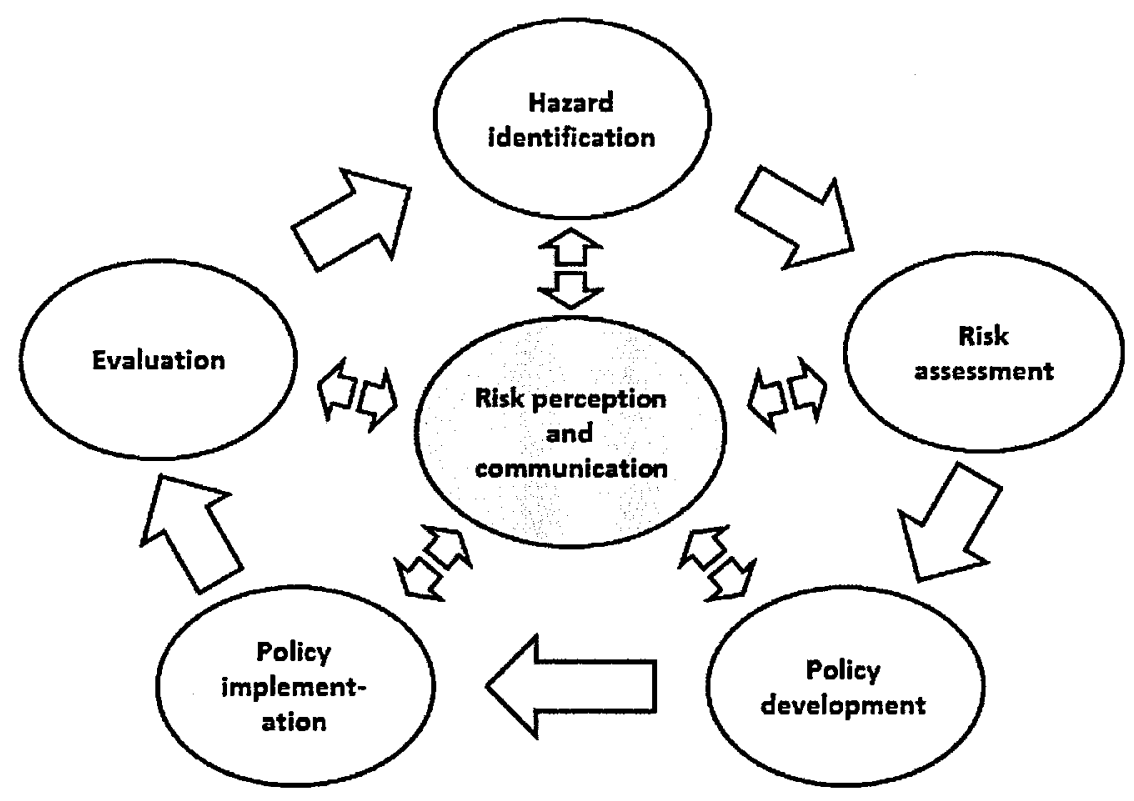

Figure 3.9: Risk management cycle (Gerrard and Petts, 1998)

\subsubsection{Perspectives on risk: Summary and conclusions}

This section has given a brief overview of risk within the contexts of business, engineering and the environment, from the three technical perspectives on risk defined by Renn (1998b). The purpose of this review was to use these different perspectives to define components of risk, which could then be used to define a perspective on risk for this research. It is possible to distinguish between the different perspectives on risk reviewed using two components, which are based on the sources and receptors model taken from the review of risk in an environmental context:

- A viewpoint of what constitutes a hazard (event) - the source;

- What receptors are of interest in terms of assessing the effects of that hazard.

Each perspective on risk is also distinguished by the terminology used to describe the various activities undertaken to understand and manage risk.

Table 3.3 provides a summary for this section, clarifying the perspectives on risk that have been reviewed using the sources and receptors model, also highlighting differences in terminology between the perspectives. The next section uses the sources and receptors model to define a perspective on risk for this research, also defining the terminology to be used here. 
Table 3.3: Summary of risk perspectives and terminology

\begin{tabular}{|c|c|c|c|c|}
\hline & $\begin{array}{l}\text { Rolls-Royce } \\
\text { (section 3.2) }\end{array}$ & $\begin{array}{c}\text { Business } \\
\text { (section 3.3.1) }\end{array}$ & $\begin{array}{c}\text { Engineering } \\
\text { (section 3.3.2) }\end{array}$ & $\begin{array}{c}\text { Environment } \\
\text { (section 3.3.3) }\end{array}$ \\
\hline \multicolumn{5}{|c|}{ Summary of risk perspectives } \\
\hline $\begin{array}{r}\text { Risk } \\
\text { definition: }\end{array}$ & $\begin{array}{l}\text { 'threats to the } \\
\text { achievement of } \\
\text { business } \\
\text { objectives or the } \\
\text { continuing } \\
\text { reputation of the } \\
\text { group' }\end{array}$ & $\begin{array}{c}\text { 'effects of } \\
\text { uncertainty on } \\
\text { objectives' }\end{array}$ & $\begin{array}{l}\text { 'the probability that } \\
\text { an untoward event } \\
\text { will happen, } \\
\text { multiplied by the } \\
\text { impact it could } \\
\text { have if it did } \\
\text { happen' }\end{array}$ & $\begin{array}{l}\text { 'a combination of } \\
\text { the probability, or } \\
\text { frequency, of } \\
\text { occurrence of a } \\
\text { defined hazard and } \\
\text { the magnitude of } \\
\text { the consequences } \\
\text { of the occurrence' }\end{array}$ \\
\hline Sources: & $\begin{array}{l}\text { Determined } \\
\text { through initial } \\
\text { 'planning' step } \\
\text { and sources of } \\
\text { knowledge used } \\
\text { to identify risks }\end{array}$ & $\begin{array}{l}\text { Determined } \\
\text { through } \\
\text { 'establishing the } \\
\text { context' }\end{array}$ & $\begin{array}{l}\text { Engineering } \\
\text { failures, related to } \\
\text { products and } \\
\text { processes }\end{array}$ & $\begin{array}{l}\text { Defined by } \\
\text { conceptual model }\end{array}$ \\
\hline Receptors: & $\begin{array}{l}\text { Business } \\
\text { objectives }\end{array}$ & $\begin{array}{l}\text { Business } \\
\text { objectives }\end{array}$ & $\begin{array}{l}\text { Business } \\
\text { performance, } \\
\text { human health } \\
\text { and/or the } \\
\text { environment }\end{array}$ & $\begin{array}{l}\text { Environment and } \\
\text { human health }\end{array}$ \\
\hline \multicolumn{5}{|c|}{ Summary of risk terminologies } \\
\hline $\begin{array}{r}\text { Risk } \\
\text { identification: }\end{array}$ & $\begin{array}{l}\text { Recording } \\
\text { events that may } \\
\text { impact on the } \\
\text { objectives of an } \\
\text { activity }\end{array}$ & $\begin{array}{l}\text { Same definition } \\
\text { as within Rolls- } \\
\text { Royce, although } \\
\text { included as part } \\
\text { of 'risk } \\
\text { assessment' }\end{array}$ & $\begin{array}{l}\text { Hazard or risk } \\
\text { identification }\end{array}$ & $\begin{array}{c}\text { Hazard } \\
\text { identification }\end{array}$ \\
\hline $\begin{array}{r}\text { Risk } \\
\text { assessment: }\end{array}$ & $\begin{array}{l}\text { Assessing the } \\
\text { likelihood and } \\
\text { potential impact } \\
\text { of a risk and } \\
\text { calculating the } \\
\text { product of the } \\
\text { two to evaluate } \\
\text { significance }\end{array}$ & $\begin{array}{l}\text { Included within } \\
\text { risk analysis and } \\
\text { risk evaluation }\end{array}$ & $\begin{array}{l}\text { Same as Rolls- } \\
\text { Royce, although } \\
\text { evaluating } \\
\text { significance can be } \\
\text { included within risk } \\
\text { management }\end{array}$ & $\begin{array}{l}\text { Same as Rolls- } \\
\text { Royce, although } \\
\text { evaluating } \\
\text { significance can be } \\
\text { included within risk } \\
\text { management }\end{array}$ \\
\hline $\begin{array}{r}\text { Risk } \\
\text { treatment: }\end{array}$ & $\begin{array}{l}\text { Actions taken to } \\
\text { reduce risks to } \\
\text { an acceptable } \\
\text { level }\end{array}$ & $\begin{array}{c}\text { Same as Rolls- } \\
\text { Royce }\end{array}$ & $\begin{array}{l}\text { Part of risk } \\
\text { management }\end{array}$ & $\begin{array}{l}\text { Part of risk } \\
\text { management }\end{array}$ \\
\hline $\begin{array}{r}\text { Risk } \\
\text { management: }\end{array}$ & $\begin{array}{l}\text { Used to } \\
\text { describe the } \\
\text { overall set of } \\
\text { activities and } \\
\text { processes used } \\
\text { to manage risk }\end{array}$ & $\begin{array}{c}\text { Same as Rolls- } \\
\text { Royce }\end{array}$ & $\begin{array}{l}\text { Focuses on } \\
\text { evaluating the } \\
\text { significance of } \\
\text { risks and treatment } \\
\text { actions required }\end{array}$ & $\begin{array}{c}\text { Focuses on } \\
\text { evaluating the } \\
\text { significance of risks } \\
\text { and treatment } \\
\text { actions required }\end{array}$ \\
\hline
\end{tabular}

\subsubsection{The perspective on risk for this research}

The aim of this chapter was to investigate how risk could be used to integrate environmental considerations into design decisions in Rolls-Royce. Chapter 2 outlined how risk management forms an important part of design within Rolls-Royce, which can be classified as a subset of risk management within a business context. Hence, using 
risk to integrate environmental considerations into design decisions infers a perspective focused on assessing risks within the frame of business risk management, although with a specific emphasis on how the environmental impacts of business activities can pose a risk to business objectives. Using the sources and receptors model, effectively this defines the receptor as the business and hazards as environmental impacts. This perspective on risk is very similar to that of Sharratt and Choong (2002), although with a more general interest in business risks rather than focusing on the specific context of process industry projects. To make it clear, this adopts a different perspective to risk in the environmental context, as the object of concern is assessing business risks, not risks to the environment.

A perspective on risk that views environmental impacts as hazards that can impact on business objectives is not new. Matten (1995) observed such a relationship, identifying the components of environmental risk as shown in Figure 3.10. Matten also observed that environmental risks do not come from the environment itself, but the response of a social group or stakeholder to an environmental impact. This approach defines stakeholders as anyone who can influence, or is influenced by, the activities of an organisation (Earl and Clift, 1999; Matten, 1995).

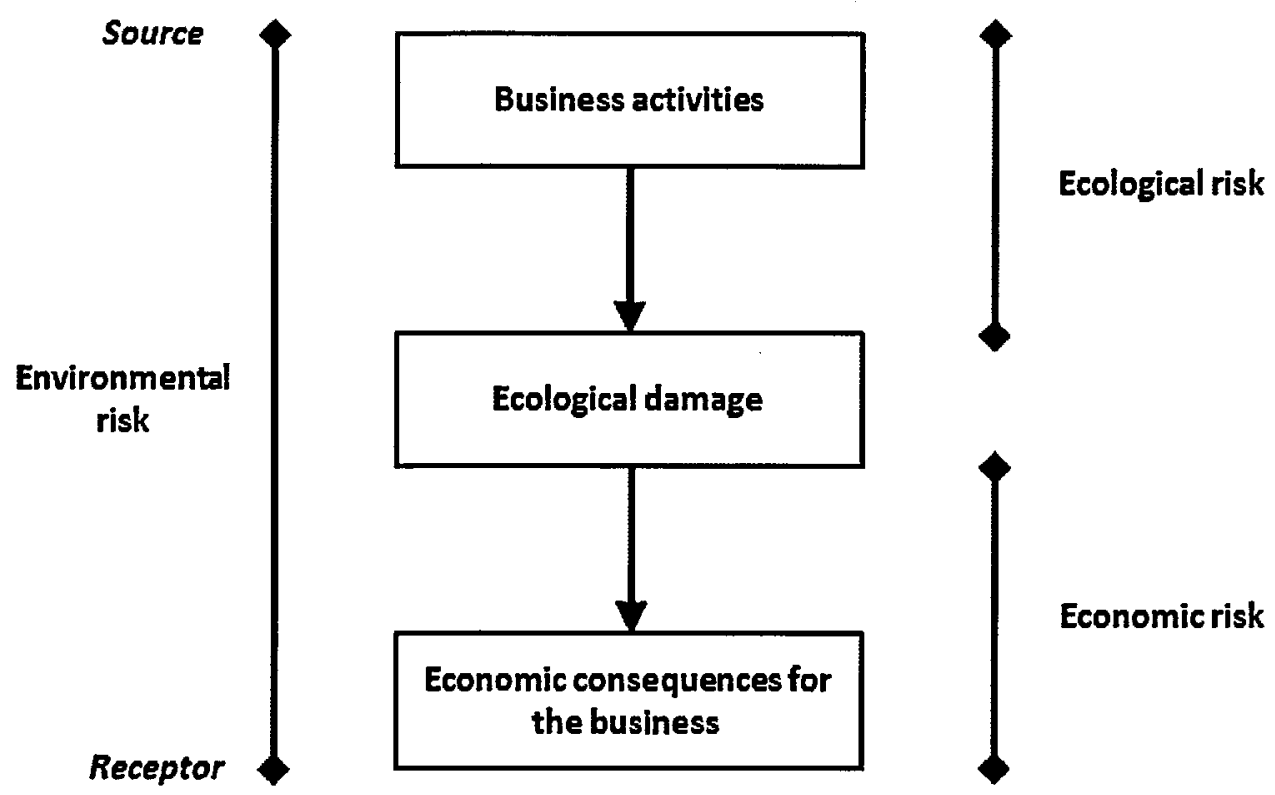

Figure 3.10: Components of environmental risk (adapted from Matten, 1995)

It is possible to see how environmental impacts create business risks. For example, environmental impacts can be the subject of regulation, or can be relevant to reputational risks, as 'environmental concerns also pose a direct challenge to firms' reputations and brands' (Ernst \& Young, 2009, p. 18). However, how environmental impacts give rise to business risks is sometimes not obvious. Stakeholder responses can come from a complex mixture of actual, potential or perceived environmental 
impacts and be influenced by dynamic political factors and societal values (Matten, 1995). It is not surprising that sometimes organisations can overlook these types of problems (see for example, Elkington and Trisoglio, 1996).

It is desirable for an organisation to begin to look beyond its immediate business activities and consider environmental impacts throughout the entire life cycle of its products (Remmen et al., 2007), or across its 'value chain' (Ernst \& Young, 2009). It is conceivable that environmental impacts across the life cycle of a business' products can lead to impacts on business objectives. However, considering risks posed by life cycle environmental impacts presents a complex task, as cause and effect relationships might not be immediately obvious, increasing the chances that these types of problems become overlooked. As noted by Sharratt and Choong (2002), solving this problem also requires a method for assessing the environmental impacts of a business over the product life cycle and LCA is a tool that can fulfil this requirement.

It is important to define the risk terminology that will be used within this risk perspective. For the purposes of this research, the following risk terms are defined, building on the components of environmental risks defined by Matten (1995):

- Environmental business hazard: stakeholder responses to environmental impacts with the potential to cause harm to business objectives;

- Environmental risk: multiplying the probability of an environmental business hazard by its potential impact on business objectives;

- Environmental risk management: the activities of identifying, assessing and treating environmental risks.

\subsubsection{Defining risk: Summary and conclusions}

This section has defined the perspective on risk to be applied for this research and the terminology to be used. Referring back to the research theme for this chapter, what now requires investigation is the potential benefits of managing environmental risks (as defined) like any other risk as part of standard design risk management activities within Rolls-Royce. To define how this can be achieved the next section builds the framework for environmental risk management. For the purposes of this research, a framework is considered to be a means of visually representing concepts, features, processes, and tools (generically termed 'elements' of a framework) and their interrelationships necessary to give the desired outcome, which is an assessment of the environmental risk posed to a design. This is similar to the descriptions of a framework from Robson (1993) and DETR (2000). 


\subsection{The framework for environmental risk management}

This section defines the various elements that need to go into a framework to assess the environmental risks posed to a design, building visual representations of their interrelationships to develop the framework for environmental risk management. The description of the framework for environmental risk management is developed from Lloyd et al. (2012a).

To assess the environmental risks posed against a design, the first two elements that can be used to build up the framework are:

- The design process, where it is desired to integrate consideration for environmental risks, in particular the elements of the standard design framework outlined in chapter 2;

- Environmental business hazards, as defined in section 3.3.

Figure 3.11 presents a visual representation of the design process and environmental business hazards, using an arrow to indicate that in order to assess the environmental risk posed to a design it is necessary to link these two elements together using information that establishes a connection between a hazard and a design.

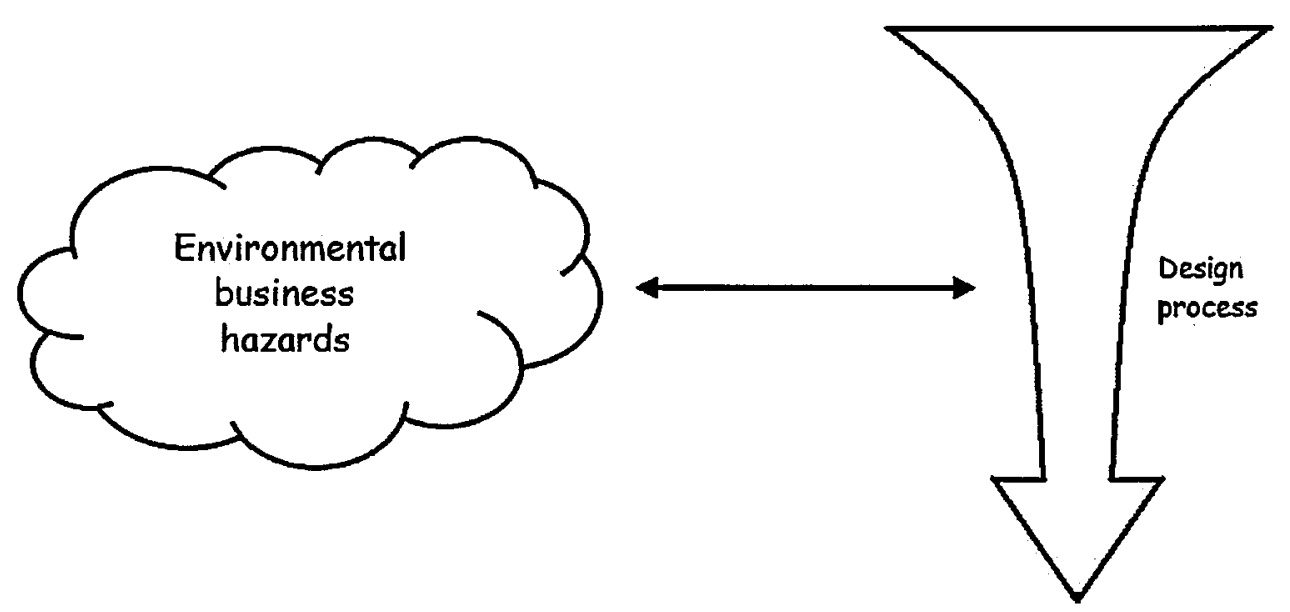

Figure 3.11: Environmental business hazards and the design process

Figure 3.11 immediately defines two perspectives on the problem of assessing environmental risks. From the perspective of a designer within the design process, in undertaking design activities a physical product solution will materialise to fulfil the defined requirements, which will cause environmental impacts across the product life cycle. Of concern is identifying which of these environmental impacts will lead to stakeholder responses that could impact on business objectives. Referring back to the components of environmental risk as defined by Matten (1995) (Figure 3.10), this 
perspective effectively works from the top of Figure 3.10 downwards, or from right to left in Figure 3.11.

Another way of looking at this problem is considering more broadly how stakeholder responses to environmental impacts could impact on business objectives, the problem is identifying how these environmental business hazards may impact on the existing and future product portfolio. In order to do this it will be necessary to link these hazards to the environmental impacts of products. Effectively this works from the bottom of Figure 3.10 upwards, or from left to right in Figure 3.11. The framework proposed in this research captures both of these perspectives on the problem of managing environmental risks.

Drawing from the process of risk management used within Rolls-Royce discussed earlier in this chapter, in order to manage environmental risks, one of the first steps is to identify some environmental business hazards relevant to the business. From discussions with risk managers within Rolls-Royce, it was also suggested that some means of prioritising identified hazards is required, as there are likely to be many, deciding which are important enough to be considered as risks within design decisions. Building on Figure 3.11, Figure 3.12 identifies two steps within the framework, identifying and prioritising environmental business hazards. Hollow arrows are used to highlight the steps of identifying and prioritising hazards, maintaining a solid arrow to indicate that it is these prioritised hazards that need to be linked to products to assess the environmental risks posed to designs. Sufficient information on prioritised hazards will be required to substantiate a link with a product.

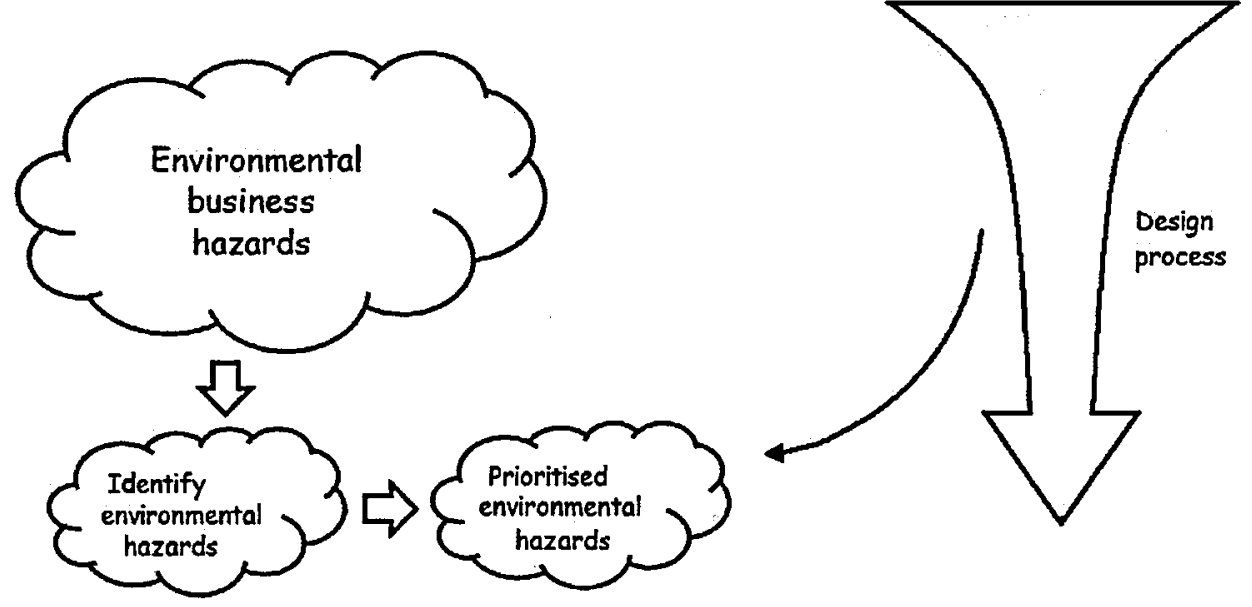

Figure 3.12: Identifying environmental business hazards

Figure 3.12 only captures the design perspective on the problem of assessing risks described above (from right to left in Figure 3.11). To capture how environmental business hazards may impact on the existing product portfolio (from left to right in 
Figure 3.11), it will also be necessary to consider how prioritised environmental business hazards impact on products that have already been designed and are in service. Figure 3.13 adds in-service products, inserting a dashed arrow indicating a link between environmental business hazards and in-service products, distinguishing this perspective from the design perspective.

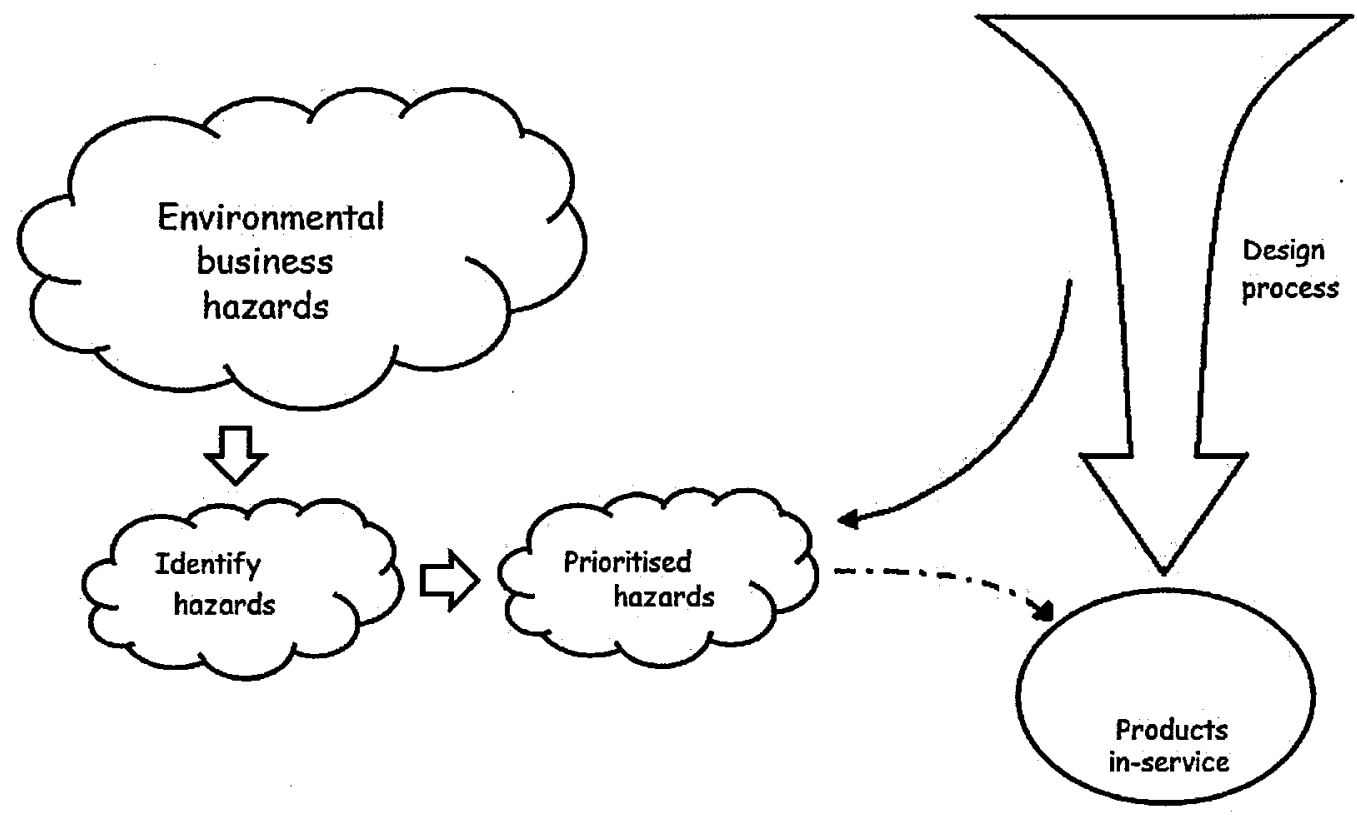

Figure 3.13: With in-service products

At this point, Figure 3.13 represents an adaptation of Figure 3.11, capturing how environmental business hazards need to be identified and prioritised and also capturing how environmental business hazards need to be considered from the two perspectives on the problem of assessing environmental risks described above. To identify which designs and products are affected by which environmental business hazards, and vice versa, requires environmental data of the products being designed and products inservice. By definition an environmental business hazard has to be related to the environmental impacts of a product. What is required is to provide sufficient information on these impacts to substantiate a link between a product and an environmental business hazard. As described in chapter 1 , the starting point for this research was the development of a methodology for producing environmental information in design, similar to a simplified form of LCA, and such a tool could fulfil the need for product environmental information required to manage environmental risks. Figure 3.14 incorporates 'Life Cycle Environmental Data' (LCED) to reflect this requirement, which will need to be created for new designs and also for existing products. 


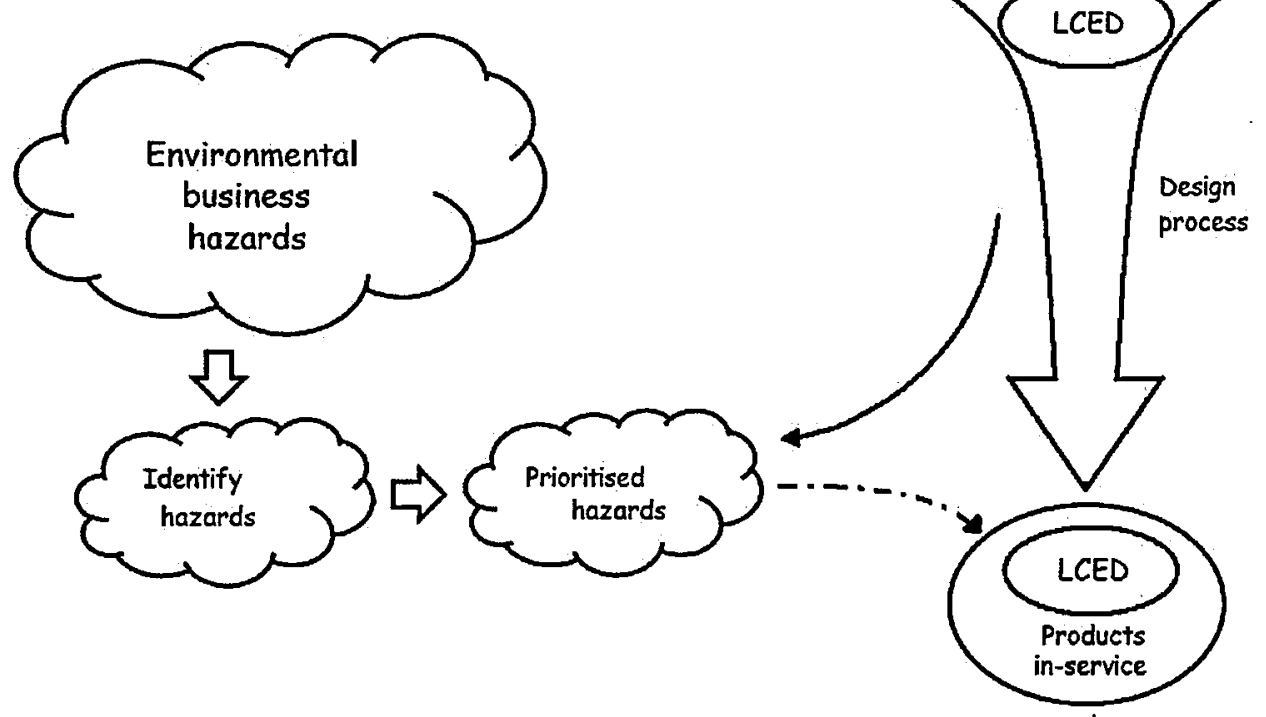

Figure 3.14: With Life Cycle Environmental Data (LCED)

As assessment of the risk posed by environmental business hazards can be made once a link is made between a hazard and a product. At this point the risks can be assessed within the standard risk management processes already in place within RollsRoyce. Figure 3.15 finishes the framework for environmental risk management by incorporating standard risk management elements covering risk assessment and treatment. 


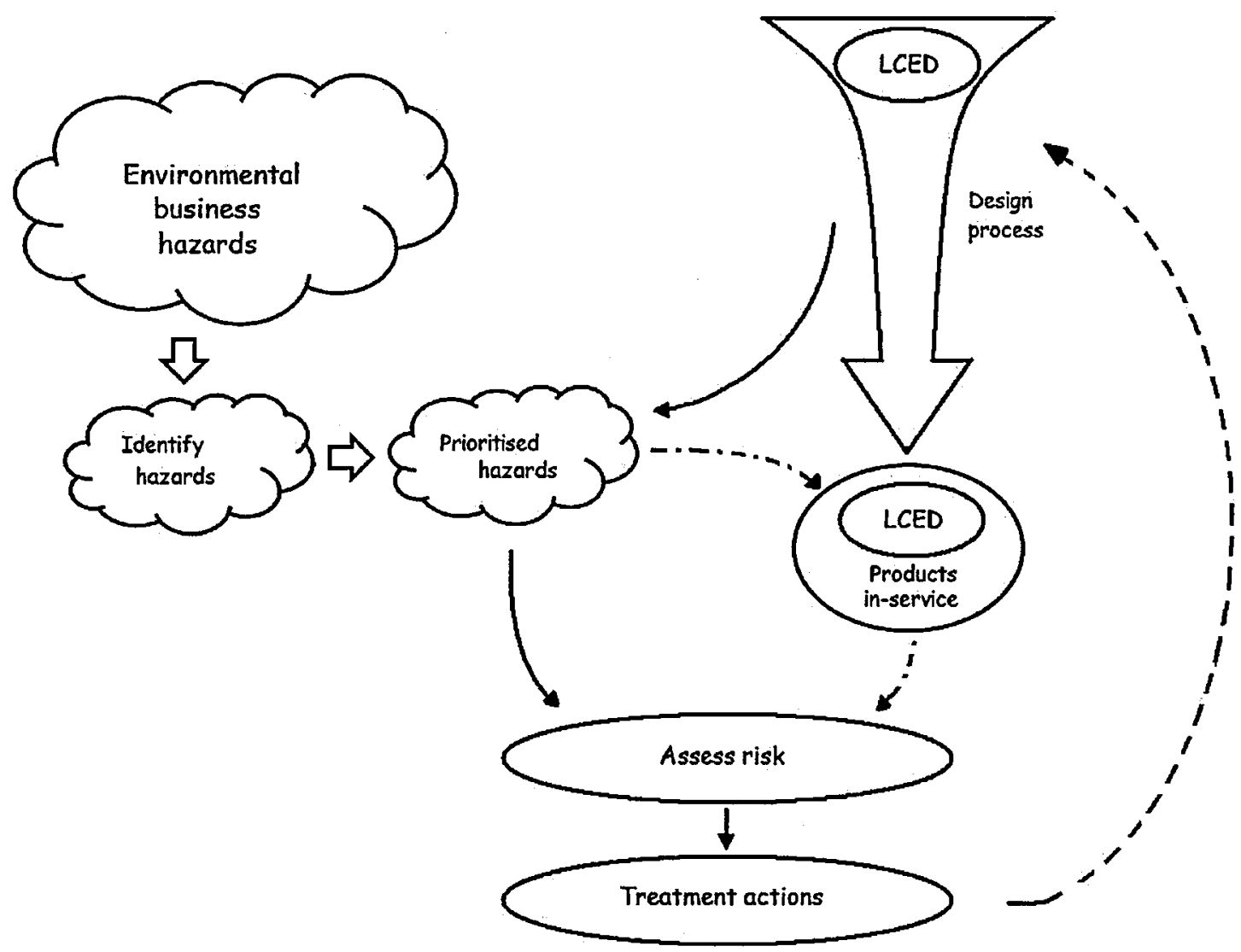

Figure 3.15: Framework for environmental risk management (developed from Lloyd et al., 2012a)

Some, but not all, of the treatment actions required to mitigate environmental risks could take place within the design process, which is highlighted with a dashed arrow on the right hand side of the diagram.

The framework presented in Figure 3.15 offers a high-level theoretical interpretation of how environmental risks could be managed in design. More detail will need to be provided on each element of the framework, which is explored as the framework is tested through pilot research activities presented in the following chapters.

\subsection{Summary and conclusions}

This chapter has presented the research activities undertaken to investigate how risk could be used to integrate environmental considerations into design decisions, developing the framework for environmental risk management. Risk management processes within Rolls-Royce were reviewed in section 3.2. This review concluded that environmental risks should be managed like any other risk within the standard RollsRoyce risk framework and it was required to investigate the topic of risk more thoroughly to understand precisely the types of risks this research sought to manage. 
A review of risk management literature followed in section 3.3. The source-receptor model taken from a review of risk in an environmental context was used to define a perspective on risk for this research, which effectively defines the receptor as the business and hazards as environmental impacts that arise from business activities. It was also discussed how it was necessary to not only consider how environmental impacts from business activities pose business risks, but also environmental impacts across the product life cycle. The following risk terminology was defined to describe this perspective on risk:

- Environmental business hazard: stakeholder responses to environmental impacts with the potential to cause harm to business objectives;

- Environmental risk: multiplying the probability of an environmental business hazard by its potential impact on business objectives;

- Environmental risk management: the activities of identifying, assessing and treating environmental risks.

It was concluded that what this research needed to achieve was to develop a framework for ensuring environmental risks were considered like any other risk within standard design processes. Section 3.4 developed the framework for environmental risk management (Figure 3.15 ), by outlining the various elements that are required to manage environmental risks and developing a visual interpretation of their interrelationships.

Now the framework has been developed, the next challenge is to test the framework through pilot research activities, showing how it can be used to consider environmental risks as part of standard design risk management processes. As discussed during the description of the framework, one of the first steps in managing environmental risks is identifying environmental business hazards. 


\section{Identifying environmental business hazards}

This chapter presents the first set of research activities undertaken to test the framework for environmental risk management and focuses on the problem of identifying environmental business hazards. Section 4.1 reviews the research methods that were considered for identifying environmental (business) hazards, justifying the selected approach. Based on the selected approach, the research design for identifying environmental business hazards is presented in section 4.2 . Section 4.3 presents results, leading to a discussion in section 4.4. Conclusions for this chapter are provided in section 4.5 .

\subsection{Methodology}

Testing the framework for environmental risk management represented a new research theme and cycle of action research, following on from defining the framework in chapter 3. Within this research theme, action within this iteration of the research cycle focused on identifying environmental business hazards, which is the first problem that needed to be addressed to test the framework for environmental risk management (Figure 4.1).

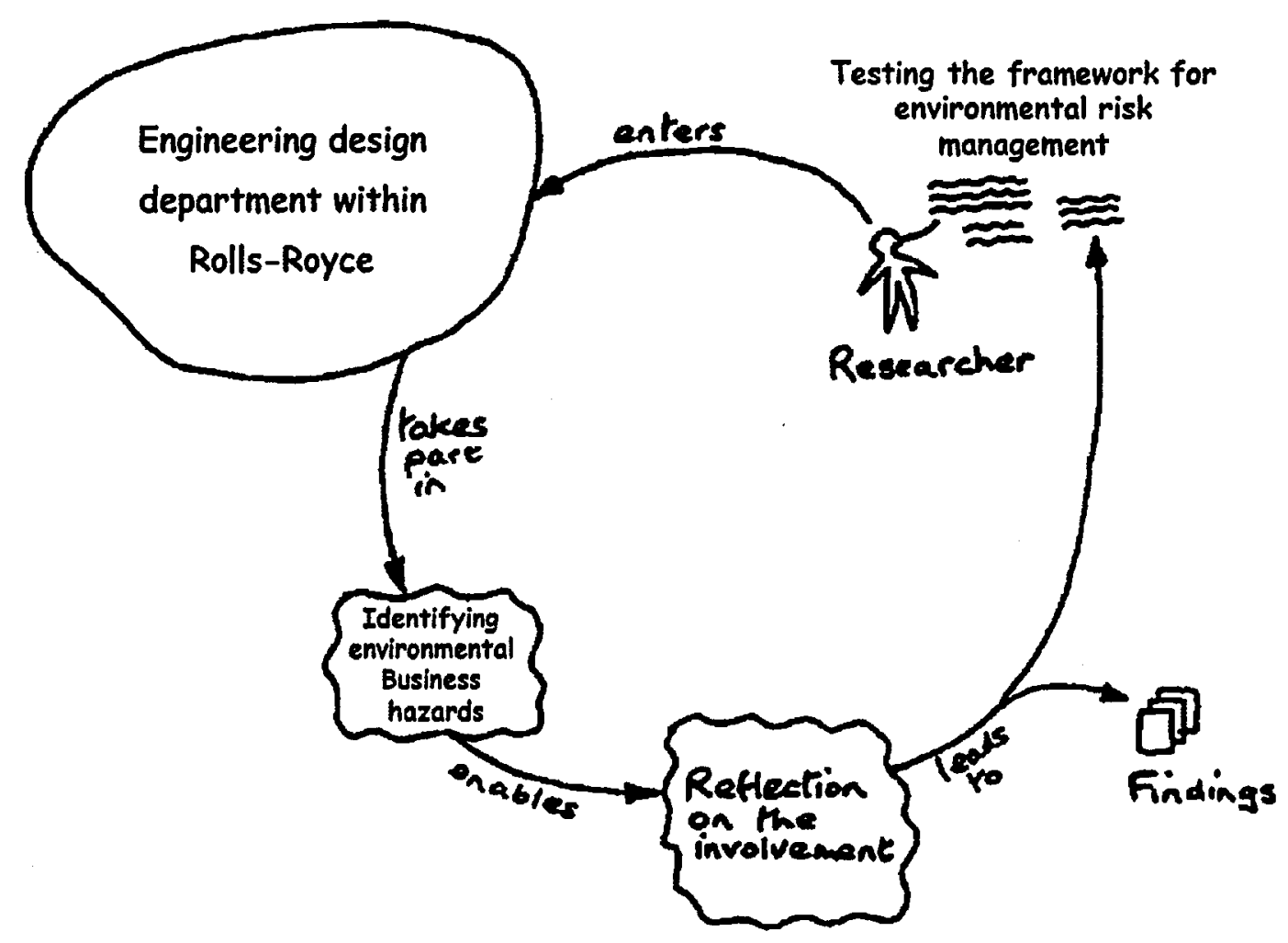

Figure 4.1: Action research cycle for identifying environmental business hazards (adapted from Checkland and Holwell, 1998) 
The aim of this part of the research was to produce a list of hazards that have been shown to be of concern to Rolls-Royce, which can then be used to test the remainder of the framework through further research. The purpose was not to identify a definitive list of hazards that are claimed to be the most important for Rolls-Royce to manage, as this would have been difficult to prove beyond reasonable doubt given the size and diversity of the organisation.

Identifying hazards focuses on testing the left hand side of the framework shown in Figure 3.15, from which two problems needed to be addressed:

1. Broadly identifying hazards, as they have been defined in chapter 3;

2. Deciding which of the hazards identified are a priority and need to be managed in design, as there could be many, which may be perceived to be of a lesser or greater importance to the business.

Prioritised hazards can be linked with products to test the remainder of the framework, as described in the development of the framework at the end of chapter 3.

Identifying hazards required the selection of a research method that was appropriate for tackling the problems posed, whilst bearing in mind the practical difficulties that were present when researching in-context within an organisation. An important consideration was the nature of environmental business hazards, which had implications for how they could be identified. From the nature of environmental business hazards described in chapter 3 (section 3.3.5), pertinent characteristics that influenced the choice of research method for identifying them included:

- Their origin as a 'stakeholder response' that occurs outside of the organisation, requiring an outward looking view;

- The fact that hazards, by their very nature, are future uncertainties, requiring a forward looking view. The time horizon used needed to be suited to RollsRoyce's products, which can have a life cycle of many decades (Lee, 2006);

- The need for the hazard to be related to Rolls-Royce's products and their life cycle environmental impacts. If they are not then they cannot impact on Rolls-Royce's business objectives;

- Hazards identified also need to be relevant to the tactical design focus defined in chapter 2 ;

- It was also important that the hazards identified are shown to be a concern for Rolls-Royce. By definition, a hazard must have the potential to lead to undesirable consequences, which can only be defined by the business itself. 
To address the two problems posed, bearing in mind the nature of environmental business hazards, it was decided to select a research approach that would capture the views of employees within Rolls-Royce on what environmental business hazards exist and their relevant importance. Social research methods geared towards capturing judgements within a business were identified as an appropriate means of achieving this. Two possible approaches considered were interviews and focus groups (Gilbert, 2001; Krueger and Casey, 2009). Methods concerned with looking into the future were also identified as being potentially suitable, given the need for a forward looking view. Two commonly used techniques considered were the Delphi method (Linstone and Turoff, 1975) and scenario planning (Lempert et al., 2009). The next sections review the four possible research methods for identifying hazards. Justifications for the selected approach are provided in section 4.1.5.

\subsubsection{Interviews}

Interviews are one of the most widely used methods for social research (Fielding and Thomas, 2001). Interviews have already been applied extensively, although informally, as a research method during the course of this research. Reflecting their use as a tool for preliminary research, a lot of these informal interviews have been used to gain an understanding of the context of the business and problem, in order to guide research direction. The question here was whether or not interviews could have been applied in a more structured way to address the problem of identifying environmental business hazards.

Interviews are generally applied as a research method in one of three different ways (Fielding and Thomas, 2001; Warren 2001):

- Structured: Questions are set within an interview schedule, which is strictly adhered to. The schedule can consist of open or closed questions, although no deviation from the schedule is permitted. Structured interviews are closely aligned to surveys as a research method, often involving the quantitative analysis of data from a more positivistic perspective of knowledge generation.

- Unstructured: Also called qualitative or focused interviews. Unstructured interviews are more of a 'guided conversation' (Warren, 2001, p.85). An interview guide is applied, which does not necessarily stipulate specific questions in order. Instead areas of interest are listed that can be covered during the interview. Judgements regarding the forming of specific questions and the order in which they are presented are left to the 
interviewer. Quite often the responses of the interviewee will be used to guide the direction of the interview. Unstructured interviews are more closely aligned with ethnography and tend more towards a interpretivist perspective of knowledge generation.

- Semi-structured: A combination of the two. Generally applied where specific issues need to be discussed in a structured way, although some freedom is required to follow interesting lines of enquiry (O'Hare, 2010).

Designing interview research can generally be broken down into the following steps (from Fielding and Thomas, 2001; Simmons, 2001; Warren, 2001):

1. Defining the topic of interest and how it can be explored using interviews;

2. Designing the interview research, including developing the interview schedule or guide and finding respondents. Topics for exploration will need to be defined along with questions. Care is required to ensure the questions are relevant to the topic of interest. Open or closed questions can be used in structured interviews. In qualitative interviews, where the aim is to gather underlying opinions that might require a frank response, open questions are likely to yield better results;

3. The interview process itself, including practical considerations of time, location and a means of gathering data, for example using recording equipment;

4. Analysis of data. For structured interviews this can be done qualitatively or quantitatively, depending on the nature of the questions posed. Unstructured interviews will require the transcription of recorded data for qualitative analysis.

Considering the use of interviews as a research method for identifying hazards, the first problem would be to decide what style of interview would be appropriate. O'Hare (2010) applied semi-structured interviews when investigating drivers and barriers to eco-innovation within electronics companies. The semi-structured approach was applied to provide an element of repeatability in the research, although with the flexibility to explore should the need arise. This suggests that a semi-structured approach would be appropriate for identifying environmental business hazards; a structured approach could be applied to introduce the concept of environmental business hazards, guiding interviewees to offer opinions. Responses could then be used to explore the topic in a more unstructured way.

Advantages of using semi-structured interviews for identifying environmental business hazards include: 
- Would remove the undue social pressures potentially present in focus groups;

- Pilot interviews could be used to develop and test a suitable interview guide;

- Interviews might be easier to organise than focus groups, as it would not be necessary to coordinate respondents into one venue at the same time;

- Structured questions could be used to guide participants who are not familiar with the topic.

Disadvantages of using semi-structured interviews to identify environmental business hazards include:

- Lack of group discussion. It is the researcher's opinion that allowing respondents to openly discuss environmental business hazards with one another will encourage debate, providing greater insight into the problem. Discussion and debate will also allow those with less knowledge of the topic to participate; environmental specialists can provide a view of the hazards to inform judgements from those with less knowledge of the topic, who potentially may have more knowledge of the business and the relevant importance of hazards.

- Interviews could not be used directly to draw a consensus on the priority of environmental business hazards, as it would only be possible to draw the opinions of interviewees individually. Responses would then need to be aggregated after completing the interviews to develop a representative opinion.

\subsubsection{Focus groups}

A focus group is an organised group interview or discussion designed to gather opinions (Cronin, 2001). Specific characteristics differentiate focus groups from other types of group discussions (Barbour and Kitzinger, 1999; Cronin, 2001; Krueger and Casey, 2009):

- A focus on particular topics related to a research problem;

- Small number of participants, generally between five and ten. Any smaller and the pool of opinions can become restricted although any bigger and the discussion can be difficult to control;

- Purposive selection of participants on the basis that they can provide information needed in order to address the research problem; 
- Use of a facilitator to guide the group discussion, generally using open questions. The facilitator can have a high or low level of moderation in guiding the discussion, or somewhere in between;

- Production of qualitative data, which is usually recorded and transcribed for analysis.

Conducting focus group research can be generally broken down into the following steps (Cronin, 2001; Krueger and Casey, 2009):

1. Defining the purpose and setting research questions that are to be explored;

2. Determining how many groups are needed and how many participants in each group. This is governed by the research question, range of people required and practical issues of time, cost and availability;

3. Selecting participants, who can provide relevant information to address the defined research questions;

4. Planning the groups, including practical issues of venue, location and arrangements for capturing data;

5. Running the groups, providing introductions, questions and setting the level of moderation between the group and facilitator;

6. Analysis of focus group data, typically using a coding scheme. A code is 'a symbol applied to a group of words to classify or categorise them' (Robson, 1993, p.385). Open coding identifies codes from within the data, with no predetermination of what the coding scheme should be (Glaser and Strauss, 1999). Closed coding involves analysing data with a coding scheme developed beforehand (Robson, 1993).

Becoming established as a research technique around the $1950 \mathrm{~s}$, initially focus groups were predominantly applied for marketing research. Since the $1980 \mathrm{~s}$ their use as a research technique has become more widespread, in particular in business, politics and academic research (Cronin, 2001). Within business they can be applied to a wide range of problems, for example product and organisational development, understanding employee concerns, customer satisfaction and policymaking and testing.

Advantages of using focus groups to identify environmental business hazards include:

- Suitable for facilitating a group of relevant participants from within RollsRoyce to discuss environmental business hazards;

- Would allow a mix of participants, including environmental specialists and managers. Specialists could provide knowledge on the hazards, allowing non-experts to participate and express opinions on their importance; 
- Group discussions are particularly valuable for consensus forming exercises (Fielding and Thomas, 2001), which is particularly relevant for addressing the second research problem of prioritising environmental business hazards that have been identified during discussions;

- Would take participants out of their daily activities and typical working environment. This would allow a focus on the problems posed and increase the likelihood of new thoughts and opinions being shared;

- Use of a facilitator could help remove the influence of researcher bias.

Disadvantages of using focus groups to identify environmental business hazards include:

- Group dynamics may stifle participation, especially if participants have varying levels of responsibility within the business;

- Might not be suitable for decision-making (Krueger and Casey, 2009), which might be necessary to draw judgements on the importance of environmental business hazards identified.

\subsubsection{Delphi method}

Linstone and Turoff (1975, p.3) define Delphi as 'a method for structuring a group communication process so that the process is effective in allowing a group of individuals, as a whole, to deal with a complex problem.' Delphi is typically based on the use of questionnaires, which are sent by a monitor group to a larger respondent group whose views are sought on the problem. Feedback is given anonymously to inform respondents of the views of others. The process of questionnaire and feedback is repeated for a number of iterations which are typically spread over four phases (Linstone and Turoff, 1975; Rowe and Wright, 1999):

1. Exploration, which is relatively unstructured allowing respondents to provide information relevant to the problem;

2. Reaching stability in group views of the problem;

3. Evaluation to allow an understanding of significant differences of opinion, if they exist, providing the opportunity for respondents to change their answers in light of group responses;

4. Aggregation of the final results, which is generally done statistically.

Delphi originally developed as a strategic forecasting tool with the objective of reaching consensus amongst experts (Linstone and Turoff, 1975). In particular it was seen as an alternative approach to statistical modelling in situations where such evaluations 
were not possible, practical or sensible (Rowe and Wright, 1999). Delphi has since developed to be applied to a wide range of decision making problems beyond statistical forecasting using quantitative data, including the capturing of qualitative information as a social science method (Linstone and Turoff, 2011). Delphi has also been applied in studies involving both qualitative and quantitative data (Tapio et al., 2011).

Delphi is generally used for practical reasons rather than for particular types of problem, as effectively the communication effort is shifted from the larger respondent group to the smaller monitoring team (Linstone and Turoff, 1975). This is particularly useful where opinions are sought from large or geographically dispersed groups that would be difficult to get together in an organised way. Anonymity also provides benefits, helping to address potential issues within group communications, for example (from Rowe and Wright, 1999):

- Domination of one or a small number of opinions or the pressure to conform;

- Disagreements between respondents affecting communication;

- Known sources of opinions affecting perceived validity, for example views expressed by those considered to be non-experts.

Discussions with a business development specialist within Rolls-Royce revealed that Delphi is a technique that has been applied as a strategic planning tool within the business. The exercise broadly followed the four phases outlined above, using initial explorations to develop hypotheses, which were then tested by soliciting expert opinions from various industrial and academic sources, iterating through feedback. These were then brought together to inform strategic business direction.

Advantages of using the Delphi method to identify environmental business hazards include:

- Would overcome the logistical problems of gaining judgements from a group of experts and managers throughout Rolls-Royce, being a geographically dispersed business;

- Often used for looking into the future, as is required here;

- Anonymity might provide the opportunity for respondents to express views that they might not pursue within a group discussion exercise;

- Delphi is a technique that is familiar to the business, having previously been applied as a strategic planning tool;

- Appropriate for developing a consensus view on important environmental business hazards.

Disadvantages of using Delphi to identify environmental business hazards include: 
- Lack of knowledge on the topic. Delphi is usually applied to gather opinions from a dispersed group of experts on a particular topic that is relatively well known. Identifying environmental business hazards represents a different type of problem as none of the respondents will have significant prior knowledge of environmental business hazards, a topic which can also be rather abstract. Participants might struggle to engage in a study without some prior knowledge. However, providing prior knowledge might influence opinions, affecting results.

- Need for a monitoring group. The only resource available to run the study is the research engineer and it would be difficult to organise a study with the resources available to the project.

- Respondents may find it difficult to fit participating in the Delphi around their day to day activities.

\subsubsection{Scenario planning}

Scenarios can be defined as 'consistent and plausible pictures of possible future realities' (Lempert et al., 2009) or a story of alternative futures (Bishop et al., 2007). Scenario planning describes the tools and techniques used to develop and apply scenarios for business and policymaking decisions (Bishop et al., 2007). In an increasingly complex world it would be an oversight for organisations and governments not to think about the future. Scenarios provide a way of doing this, analysing what is possible, probable or desirable, without claiming to be a prediction or a forecast. These scenarios of what could happen can then be used to inform strategic direction. There is a paradox in that whatever may happen in the future, it is inevitably influenced by thinking about it (Godet and Roubelat, 1996).

There are many different scenario planning techniques, appropriately described as a 'methodological chaos' (Martelli, 2001, p.62). Bishop et al. (2007) note that there are more than 20 different techniques related to scenarios and there are even different ways of categorising them. Bradfield et al. (2005), Börjeson et al. (2006) and Van Notten et al. (2003) each offer a differing typology, based on either the origins of the various techniques, scenario project goals, the scenario process, content, or user's needs related to what will, can or is desired to happen. Bishop et al. (2007) also note that, despite there being so many different techniques, the approach developed by Pierre Wack at Shell during the 1970s, presented by Schwartz (1991), is by far and away the most widely used by consultancies and organisations. The technique involves eight steps (from Schwartz, 1991): 
1. Define the key questions that need to be answered or decisions that need to be made. In this case this would be the defined research questions.

2. Find key external issues that are driving the problem. For example, growing awareness of environmental problems.

3. Identify the driving forces. This could relate to specific environmental problems, for example global warming, or factors that contribute to global warming, for example emissions from aero engines.

4. Ranking by importance and uncertainty. Deciding which of the issues identified are likely to have a significant impact on the business.

5. Creating and selecting the scenario logics. Considering how the key issues identified could be combined or related, for example continued high growth in air travel coupled with actions to significantly reduce emissions of carbon dioxide.

6. Fleshing out the scenarios. What would the world look like if growth in air travel continued whilst carbon emissions were being significantly reduced?

7. Implications. How do strategic options stand-up to the world envisaged?

8. Selection of leading indicators and signposts. Developing a means of assessing whether or not any scenarios developed are coming close to reality, for example by defining key events.

Scenarios are used for long term strategic planning, typically seeking to look longer than ten and less than fifty years into the future, which would broadly cover the life cycle of an aero engine design. Obviously different businesses and organisations are interested in different external factors and seek to apply scenarios for different reasons. However, all have the overall aim of using scenarios as a tool for strategic thinking. The Delphi method is also one approach for developing scenarios with the aim of long term strategic planning, as it was applied at Rolls-Royce.

Advantages of using scenarios for identifying environmental business hazards include:

- Only method that could be applied to identify environmental business hazards with a time horizon similar to the life cycle of an aero engine design. It is impossible to predict the future, although scenarios provide a means of thinking about it;

- Suitable for adopting the required outward looking view, as scenarios are concerned with external issues;

- Familiar to the business through the scenarios developed using the Delphi method. 
Disadvantages of using scenarios to identify environmental business hazards include:

- As a possible future state, scenarios are unlikely to produce tangible hazards that can be linked to a product and used to assess risk;

- Scenarios are more of a strategic tool and might not produce outcomes compatible with the focus on tactical design issues defined in chapter 2 ;

- Scenarios exercises are typically produced with the help of expert consultancies and it is unlikely that a scenario project could be feasibly completed given the time and resources available to this project.

\subsubsection{Selection of research approach}

Table 4.1 provides a summary of the advantages and disadvantages of each research method reviewed in the previous sections. When selecting a research approach, the primary consideration was choosing a research method that was appropriate for tackling the research problems posed. Practical issues related to the organisational context and resources available to the project were also considered.

Scenario planning was one method deemed to be unsuitable for identifying environmental business hazards, as it would have only produced possible future realities. To develop and test the framework for environmental risk management required a tangible stakeholder response that could form the basis of a product based risk assessment. Scenarios are also a tool for strategic thinking, which would have been unlikely to produce outcomes that are compatible with the tactical design focus required. Completing a scenario exercise would also not have been feasible given the resources available to the project.

Delphi might have been a suitable method for identifying environmental business hazards, meeting several of the criteria required for addressing the research questions posed and overcoming some of the potential practical difficulties. In particular, Delphi had advantages when it came to gaining opinions from geographically dispersed participants (Rolls-Royce is increasingly becoming an international business), allowing feedback between participants without undue social pressures, developing consensus opinion and as a tool for dealing with complex problems.

However, Delphi did have a significant disadvantage in that it has historically been developed and applied as a means of gathering opinions from experts on a topic that is relatively well known. Identifying environmental business hazards is a different type of problem, as respondents are unlikely to have specific prior knowledge of the topic, which, due to the nature of environmental business hazards, can be abstract to those 
unfamiliar with it. There were also practical concerns over the use of Delphi, as respondents might not have been able to find time to complete the exercise among their daily activities. It is also unlikely that a study could be completed given the resources available to the project. For these reasons the Delphi method was deemed to be unsuitable for identifying environmental business hazards.

Table 4.1: Summary of research methods reviewed

\begin{tabular}{|c|c|c|}
\hline Research method & Advantages & Disadvantages \\
\hline Focus groups & $\begin{array}{l}\text { - Suitable for facilitating a mixed group of } \\
\text { experts and managers to discuss } \\
\text { environmental business hazards. } \\
\text { - Group discussions are useful for } \\
\text { consensus forming exercises. } \\
\text { - Would take participants out of their daily } \\
\text { activities, allowing them to focus on the } \\
\text { problem. } \\
\text { - Use of an external facilitator could help } \\
\text { remove the influence of researcher } \\
\text { bias. }\end{array}$ & $\begin{array}{l}\text { - Group dynamics may stifle } \\
\text { participation, especially if } \\
\text { participants have varying levels } \\
\text { of responsibility within the } \\
\text { business. } \\
\text { - Might not be suitable as a } \\
\text { decision-making tool, although } \\
\text { the importance of group } \\
\text { discussion in consensus } \\
\text { forming exercises has been } \\
\text { noted. }\end{array}$ \\
\hline $\begin{array}{l}\text { Semi-structured } \\
\text { interviews }\end{array}$ & $\begin{array}{l}\text { - Would remove undue social pressures } \\
\text { potentially present in focus groups. } \\
\text { - Practically interviews might be easier to } \\
\text { organise. } \\
\text { - Structured questions could be used to } \\
\text { guide participants who are not familiar } \\
\text { with the topic. }\end{array}$ & $\begin{array}{l}\text { - Lack of group discussion. } \\
\text { - Difficult to gain a consensus, as } \\
\text { it would only be possible to } \\
\text { draw opinions of interviewees } \\
\text { individually. }\end{array}$ \\
\hline Delphi method & $\begin{array}{l}\text { - Would remove practical problems } \\
\text { associated with gaining judgements } \\
\text { from a group of experts and managers } \\
\text { across Rolls-Royce, being a } \\
\text { geographically dispersed business. } \\
\text { - Often used for looking into the future. } \\
\text { - Anonymity might provide the } \\
\text { opportunity for respondents to express } \\
\text { views they might not otherwise pursue. } \\
\text { Appropriate for consensus forming } \\
\text { exercises. }\end{array}$ & $\begin{array}{l}\text { - Delphi has not been developed } \\
\text { to tackle new problems that } \\
\text { respondents know little about. } \\
\text { It might be difficult for } \\
\text { respondents to engage in a } \\
\text { study without some prior } \\
\text { knowledge of environmental } \\
\text { business hazards. } \\
\text { - It would be difficult to complete } \\
\text { a study given the resources } \\
\text { available to the project. } \\
\text { Respondents may find it difficult } \\
\text { to fit participating in the Delphi } \\
\text { around their day to day } \\
\text { activities. }\end{array}$ \\
\hline Scenario planning & $\begin{array}{l}\text { - Only method that could be applied to } \\
\text { identify environmental business } \\
\text { hazards over the typical life cycle of a } \\
\text { Rolls-Royce product. } \\
\text { - Very suitable for adopting the required } \\
\text { outward looking view. } \\
\text { - Familiar to the business through the } \\
\text { scenarios developed using the Delphi } \\
\text { method. }\end{array}$ & $\begin{array}{l}\text { - As a possible future state, } \\
\text { scenarios will not produce } \\
\text { tangible hazards that can be } \\
\text { linked to a product and used to } \\
\text { assess risk. } \\
\text { More of a strategic tool, which } \\
\text { is incompatible with the current } \\
\text { focus on tactical design issues. } \\
\text { - A scenario project could not be } \\
\text { feasibly completed given the } \\
\text { time and resources available. }\end{array}$ \\
\hline
\end{tabular}

What was required was an approach that would take participants out of their normal daily activities, allowing them to focus on the problems posed, with opportunities for direct discussion on environmental business hazards and how they can affect Rolls- 
Royce. Group discussions have been highlighted as important in consensus building exercises and it was noted that focus groups specifically use group interactions to create results. As it was desired to gain a consensus on environmental business hazards, allowing for research participants to actively engage with each other in a group discussion was deemed essential. Group interaction is not only important for gaining consensus, but also to allow non-expert participants to take part, in particular non-specialist senior business managers who may be able to make judgements regarding the significance of the hazards identified. Interaction with other participants, and guidance from a facilitator, can help introduce the topic of environmental business hazards, allowing inclusive discussions. For this reason interviews were deemed an inappropriate method for this part of the research and focus groups were the selected approach.

As previously mentioned, scenarios are the only suitable method for identifying hazards over a time period similar to that of an aero engine design, which was listed as an initial requirement of the research approach. Given that scenarios are not suitable, identifying hazards over this time horizon might not be a realistic expectation. The time horizon over which hazards might be reasonably expected to be identified aspect was explored through the focus groups and is discussed in later sections.

\subsection{Research design}

This section outlines the focus group research design that was applied for identifying environmental business hazards. The research design for the focus groups was based around the steps identified in section 4.1.1, which were:

1. Defining the purpose, which was presented in section 4.1;

2. Determining how many groups are needed, how many participants in each group and recruiting participants;

3. Planning and running the groups;

4. Analysing focus group data.

\subsubsection{Number of groups and participant recruitment}

The following factors were considered to determine the number of groups required and to recruit participants for the focus groups:

- The need for a range of opinions to provide knowledge on environmental business hazards that might affect Rolls-Royce; 
- The need for an opinion from each CFBU within Rolls-Royce, to show the hazards identified are a genuine concern for the whole group;

- How many people should be in each group (typically between 5 and 10 , from section 4.1.1).

Bearing in mind the nature of environmental business hazards discussed earlier in this chapter, and in chapter 3 , it was sought to gather the following range of opinions to provide useful insights for identifying environmental business hazards:

- Environmental specialists who have the required knowledge of environmental issues that can affect the whole business. Rolls-Royce has a corporate environment function, which would be an obvious place to recruit participants from;

- Senior managers who can take responsibility for making judgements regarding the significance of environmental business hazards, which can be used to determine whether or not hazards require a more in-depth risk assessment;

- Engineers and designers, given the purpose of the framework is to produce hazards that can be used to influence design decisions. Participants who have a view across the whole business would be preferable, such as those who lead engineering communities of practice;

- Supply chain managers. Rolls-Royce outsources the manufacture of significant parts of its products, making a supply chain perspective important;

- Communications managers. Environmental issues can be subject to significant public scrutiny and involving those who represent the company externally would be insightful;

- Representatives from research functions, who focus a lot of time on developing new products and technologies that can offer environmental improvements;

- Corporate planners. Reviewing the use of Delphi within Rolls-Royce revealed how environmental issues had been considered within strategic decision making by the business. The researcher was interested to see how these views compared with those of other participants who are not involved in strategic planning.

To gain an opinion from each business it was necessary to recruit participants from as many business units as possible. Rolls-Royce was broadly separated into four businesses at the time of the focus groups: civil aerospace, defence aerospace, marine and energy (including the nuclear business). Aside from nuclear, all of the businesses 
core products are predominantly based on variants of gas turbine engines. It is likely that hazards will affect all gas turbine businesses, as engines are typically manufactured using similar materials and processes.

It was clearly not possible to encompass the range of opinions sought, and broad representation from across the business required, within one focus group. However, a representative set of participants covering the opinions and businesses would be satisfactory, rather than attempting to cover all viewpoints in detail. A representative opinion was more likely to provide clear results on the important hazards that could be used to test the framework and too many opinions could have obfuscated what is important. As emphasised at the beginning of this chapter, the objective was to produce a list of hazards that are shown to be of concern to the business, rather than produce results that are definitively claimed to be the most important hazards for RollsRoyce.

Bearing in mind the need to incorporate a range of opinions, whilst keeping the number of participants to a manageable level, it was decided to run two focus groups incorporating up to a maximum of ten participants in each, meaning that up to twenty participants were involved in total. Running two groups would also provide some redundancy in the research design. There was a chance that the focus groups might not run as planned, as this was the first time that such an exercise was to be completed within the business.

Existing contacts and cold-calling were used to recruit participants into the groups. Existing contacts were approached first. At the time of the focus groups the research engineer had been working within the business for almost three years. Through initial research activities a network of contacts had been established, particularly within the corporate Health, Safety and Environment (HS\&E) function, which is responsible for the management of environmental issues across the business. Several existing contacts were suitable participants for the groups and could also be used to recommend further participants based on the range of views required.

Other potentially suitable participants were cold-called. Employees with job roles that matched one or more of the required perspectives were identified through analysing Rolls-Royce's internal organisation charts and by using existing contacts. Potential participants were then telephoned, usually at a pre-arranged time, to discuss the research and whether or not they would like to participate. This usually led to recommendations towards other employees whom the person knew and thought would be more suitable, leading to further calls. All cold-calls were followed up with a 
summary email and one page outline of the research objectives. The calling strategy was successful and the majority of the group participants were recruited in this way.

Eighteen participants were recruited in total, sufficient to run the two focus groups. Participants covered all of the perspectives outlined above apart from the gas turbine parts of the energy business, which could not be represented as it is predominantly based in Canada. This does represent a gap in opinion, although given the research is being sponsored by the Defence Aerospace business unit it was decided that this gap should not stop the groups from going ahead. Job titles of the focus group participants are given in Table 4.2, including how the participants were split into two groups. Attempts were made to evenly spread participants between the groups, providing a balance of views between environmental specialists and business managers in each. However, this was restricted by some participants only being able to attend at certain times of the day due to other work commitments.

Table 4.2: Job titles of focus group participants

\begin{tabular}{|c|c|}
\hline Focus group 1 & Focus group 2 \\
\hline Head of Environmental Management & Global Chief of Systems Design \\
\hline Head of Supplier Engineering & Materials Specialist - Corporate HS\&E \\
\hline Improvements Manager - Corporate HS\&E & Engineering Purchasing Executive \\
\hline Head of Research \& Technology - Civil Nuclear & Corporate Communications Partner \\
\hline Head of HS\&E - Marine & Head of Research \& Technology - Marine \\
\hline Corporate Development Manager & Technology Strategy Manager \\
\hline Head of HS\&E - Customer Business & Head of Manufacturing \\
\hline Global Commodity Leader - Raw Materials & Executive Vice President - Civil Nuclear \\
\hline Environmental Strategist - Civil Future Programmes & Capability Lead - Life Cycle Engineering \\
\hline
\end{tabular}

\subsubsection{Planning and running the groups}

Both groups were planned to occur on the same day and were held at the main RollsRoyce business site located in Derby on the $6^{\text {th }}$ July 2010. An external consultancy was employed to facilitate the groups, record data and transcribe it for analysis. The consultants were given a detailed overview of the topic area, including the need to ensure that the group outcomes were representative of hazards that are of concern to Rolls-Royce. Group facilitation applied the following schedule:

- Introductions, of all group participants, the facilitator, and researcher, who was present in both groups;

- General discussion of environmental business hazards. After an introduction on the topic from the facilitator participants were invited to 
discuss their views on environmental business hazards and what was significant for Rolls-Royce;

- After approximately one hour of discussions participants were then invited to address the second research problem, offering judgements on the significance of environmental business hazards to develop a consensus on the important problems.

Including introductions, discussions and judgements on hazards, the group running time was just over two hours.

\subsubsection{Analysis of focus group data}

As discussed in section 4.1.1, focus group data is typically analysed using a coding scheme to produce results. In this circumstance, the main requirement for a coding scheme was to assign parts of the focus group discussions to a particular hazard or related environmental topic. Given this requirement, the use of a closed coding approach would not have been suitable, as this would have effectively identified environmental business hazards before the focus groups had been conducted. However, some form of coding scheme would be required to address the second research problem of prioritising hazards. To determine how important a hazard was relative to others required a means of assigning parts of the data to particular environmental business hazards or related topics, so those discussed the most frequently could be identified.

To address both research questions a key-phrase analysis was used combining open and closed coding approaches. Open coding was applied to identify key-phrases that either described a specific hazard or general topics of discussion related to the environment and potential impacts on Rolls-Royce's business objectives. These keyphrases were then used as a closed coding scheme, re-analysing the data to determine the significance of each phrase by how frequently it was discussed. Appendix 1 contains examples of how the key-phrase analysis was applied using excerpts from the focus group transcripts.

To provide further support for determining the important environmental business hazards, and address the second research problem, it was also decided to effectively run an initial risk screening exercise within the groups. Judgements of the severity and likelihood of hazards identified were captured to highlight those that were important, using an output chart similar to the one shown in Figure 4.2. 


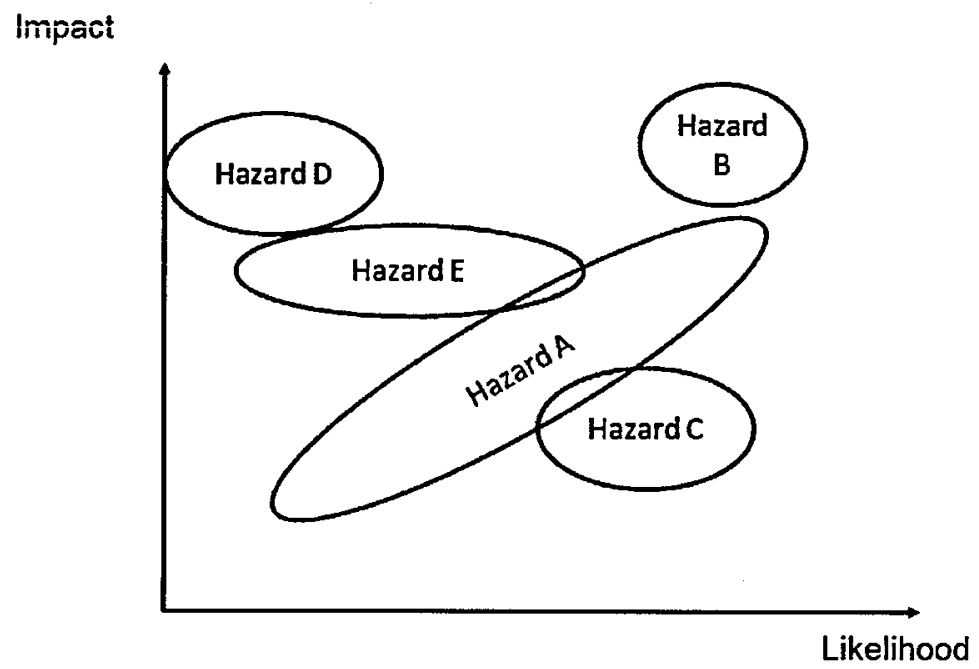

Figure 4.2: Focus group output chart

Together with the analysis of the focus group recordings, output charts were used to identify hazards deemed to be the most significant, which could be used to develop and test the framework.

\subsubsection{Role of the researcher}

It is necessary to consider how the presence of the researcher in both focus groups may have influenced the outcomes, as it was important that the focus groups showed a consensus on hazards that are of concern to Rolls-Royce (not the researcher). Throughout the whole process of conducting the focus groups the researcher fulfilled three roles:

- Recruitment of focus group participants. Recruitment through cold-calling involved the use of what was effectively a sales-pitch and potential participants inevitably asked for specific examples of what a hazard might be. Providing an answer could have potentially influenced their opinions, affecting group outcomes;

- Providing an introduction within the focus groups, with a brief explanation of the objectives of the exercise;

- Assisting the facilitator in capturing judgements on the importance of particular hazards, using the output charts described in Figure 4.2.

To ensure that the researcher had minimal impact on the outcomes of the focus groups:

- In recruiting participants, to avoid influencing opinions, generic categories of hazards were provided when asked about specific issues, for example regulation or customer requirements, highlighting the importance of the 
outcomes as a Rolls-Royce view. This tactic was also employed during the group introductions;

- During the first part of the groups, which facilitated an open discussion of environmental business hazards and how they might affect Rolls-Royce, the researcher played as minimal a role as possible, leaving control of the discussions to the external facilitator;

- When capturing judgements of the importance of hazards it was made very clear that the output charts would be used. The researcher invited participants to make a judgement of significance, involving all group members and ensuring that all decisions were agreed as a group position.

\subsection{Results}

This section presents the results from the focus group discussions. Section 4.3.1 presents results from the key-phrase analysis, listing the key-phrases identified using an open-coding approach, followed by results of how frequently each topic was discussed using closed-coding. Section 4.3.2 presents the output charts, using excerpts from the focus group transcripts to highlight relevant points. Excerpts are appropriately edited so as not to reveal the identity of participants.

\subsubsection{Key-phrase analysis}

Table 4.3 shows the list of key-phrases resulting from the first analysis of both focus group recordings, which applied the open-coding approach. 
Table 4.3: Focus group key-phrases (listed alphabetically)

\begin{tabular}{|l|}
\hline Access to resources (strategic alloying elements) \\
\hline Air quality \\
\hline Alternative fuels \\
\hline Brand and reputation \\
\hline Business continuity \\
\hline $\mathrm{CO}_{2}$ / climate change \\
\hline End of life and recycling \\
\hline Environmental opportunities \\
\hline Global producer responsibility legislation \\
\hline Local impacts \\
\hline Long life product \\
\hline Noise \\
\hline Physical effects of the environment on the business \\
\hline REACH (use of hazardous substances) \\
\hline Supply chain governance \\
\hline Sustainability \\
\hline Sustainable procurement \\
\hline Transportation costs \\
\hline Use phase CO \\
\hline
\end{tabular}

Key phrases identified covered a range of environmental topics, reflecting the broad nature of discussion that was encouraged. Some phrases covered what might be considered specific environmental business hazards, for example sustainable procurement, use phase $\mathrm{CO}_{2}$ regulation, REACH (Registration, Evaluation, Authorisation and restriction of CHemicals - or more broadly speaking the use of hazardous substances, see ECHA 2011a), and access to resources (in particular alloying elements deemed to be 'strategic' in meeting business objectives). Specific environmental impacts were also discussed, for example noise, local impacts and air quality. Features of Rolls-Royce's products and business particularly relevant to how environmental business hazards may have an effect were also picked out, for example the fact that the business makes long life products, has an extended supply chain and has a very valuable brand image. Potential business opportunities from developing environmentally better products and other topics, such as alternative fuels, were also discussed.

Table 4.4 shows the results of the second analysis using the closed coding approach to highlight key phrases that were discussed the most frequently. Each count does not represent exactly how many times each topic was mentioned, but instead represents how many passages of discussion there were related to that topic. 
Table 4.4: Frequency of key phrases (results of both focus groups)

\begin{tabular}{|l|c|}
\hline \multicolumn{1}{|c|}{ Hazard } & Count \\
\hline REACH (use of hazardous substances) & 14 \\
\hline Access to resources (strategic alloying elements) & 8 \\
\hline Use phase $\mathrm{CO}_{2}$ regulation & 6 \\
\hline $\mathrm{CO}_{2}$ / climate change and energy costs & 6 \\
\hline Brand and reputation & 6 \\
\hline Supply chain governance (ethics and management) & 4 \\
\hline Air quality & 3 \\
\hline End of life and recycling & 3 \\
\hline Alternative fuels & 3 \\
\hline Global producer responsibility legislation & 3 \\
\hline Sustainable procurement & 3 \\
\hline Long life product & 3 \\
\hline Sustainability & 2 \\
\hline Environmental opportunities & 2 \\
\hline Business continuity & 2 \\
\hline Physical effects of the environment on the business & 1 \\
\hline Noise & 1 \\
\hline Transportation costs & 1 \\
\hline Local impacts & 1 \\
\hline
\end{tabular}

REACH was clearly the most popular topic of discussion, being discussed almost twice as frequently as any other hazard. The next most popular topic was access to resources, although this was discussed only slightly more frequently than hazards related to $\mathrm{CO}_{2}$ emissions and climate change, in particular how energy costs and use phase $\mathrm{CO}_{2}$ regulation may impact the business. How environmental issues posed a hazard to the Rolls-Royce brand and reputation was also a relatively popular topic of discussion.

\subsubsection{Output charts}

Output charts were used to capture judgements on the significance of the hazards identified from the first half of the focus group discussion. An individual chart was produced for each group, discussed in the following sub-sections. During group discussions it was highlighted how it was known that some hazards identified would impact the business, rather than it being simply a measure of likelihood. To capture this, the $x$-axis of the output charts was modified to capture both likelihood and the timescale over which a hazard might affect the business. 


\subsubsection{Focus group one}

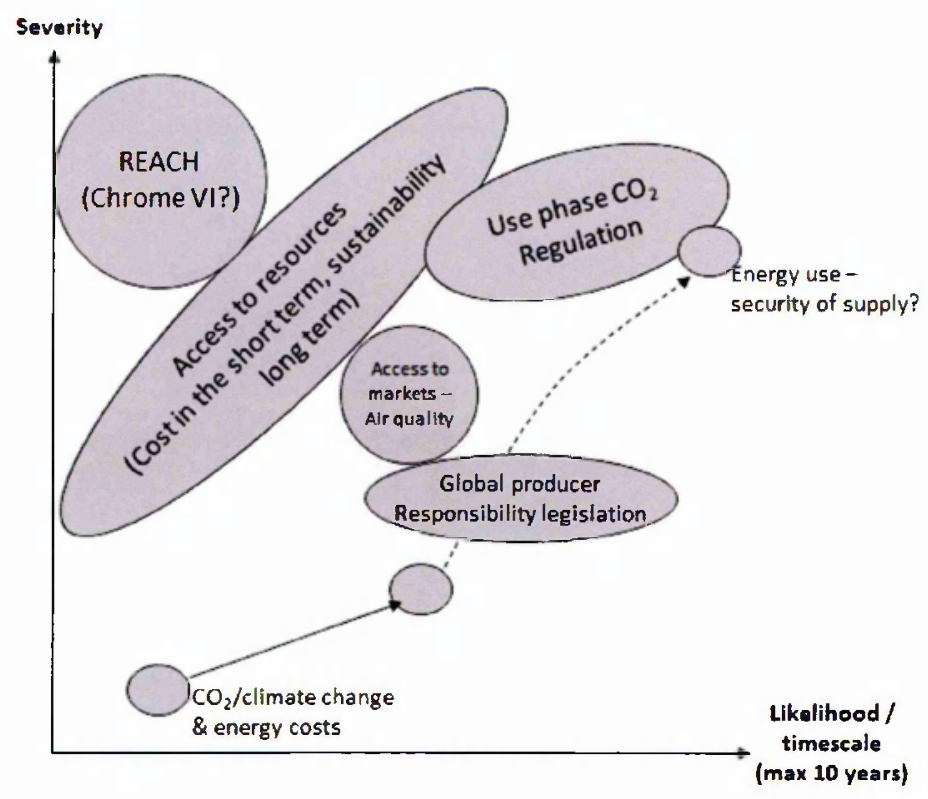

Figure 4.3: Output chart from focus group one

Figure 4.3 shows the output chart from the first focus group. Participants in the first group picked out the following hazards for the screening exercise, including whether the hazard was judged using likelihood or the timescale over which it might impact the business:

- REACH (timescale);

- Access to resources (strategic alloying elements) (timescale);

- $\mathrm{CO}_{2}$ /climate change and energy costs (likelihood);

- Air quality (likelihood);

- Global producer responsibility legislation (likelihood);

- Use phase $\mathrm{CO}_{2}$ regulation (likelihood).

REACH was deemed to be a severe and imminent threat to the business, highlighted from the opening discussion, captured in the following excerpt involving the Head of Supplier Engineering (Supplier):

$\begin{array}{ll}\text { Facilitator: } & \text { OK when you wake up at four am in the morning with a } \\ & \text { light sweat on your brow what is it you're worried about? } \\ \text { Supplier: } & \text { From my point of view business continuity as a result of } \\ & \text { REACH regulations. }\end{array}$

At the time of the focus groups some parts of the regulation had already come into effect. Particular concerns were expressed over likely restrictions in the use of substances that contain compounds with chromium in its +6 oxidation state (hexavalent chromium). Hexavalent chromium is a known carcinogen, which is one criteria defining a Substance of Very High Concern (SVHC) under REACH, and is widely used in Rolls- 
Royce's products and processes, in particular for corrosion resistant coatings. The following excerpt, including the Head of Supplier Engineering (supplier) and Head of Environmental Management (environment) illustrates the groups thoughts on how restrictions imposed by REACH would have a significant impact on the business:

$\begin{array}{ll}\text { Environment: } & \text { It's [REACH] restriction by regulation... with an intent to } \\ \text { reduce the impact on the environment and health, that's } \\ \text { the driver. } \\ \text { Supplier: } & \text { So there's lots of unintended consequences [from } \\ & \text { REACH]. } \\ \text { Environment: } & \text { Oh there is, oh I mean there is. } \\ \text { Supplier: } & \text { I was talking to an engineering fellow who sits behind me } \\ & \text { about the effects of banning chrome VI and he was } \\ \text { aghast almost and he's a rotatives specialist because } \\ \text { they use it as a sacrificial coating on the shaft and I think } \\ \text { it's important that we make the leap from them banning a } \\ \text { substance to well actually what's the effect on our product } \\ \text { and there could be actually quite a significant business } \\ \text { risk. }\end{array}$

(Engineering Fellows are senior engineering specialists within Rolls-Royce, who have responsibility for a particular engineering subject matter).

Access to resources was deemed to be a moderately severe threat in the short term, becoming more severe as time progresses, represented by the elongated bubble stretching towards the top right hand corner of the chart in Figure 4.3. In the short term impacts to cost were a concern. In the longer term broader sustainability concerns were raised, in particular how combinations of a lack of supply capacity due to materials being produced as a by-product, market monopolies and broader social responsibility issues in supplying regions may limit the accessibility of materials. These concerns are summarised in the following excerpt involving the facilitator, Head of Environmental Management (environment) and Global Commodity Leader - Raw Materials (materials), which followed on from previous discussions focusing specifically on potential supply problems associated with rhenium:

Facilitator: $\quad$ Can I go back to the rhenium thing...one thing that hasn't been discussed in that brief exchange was finding substitutes for it which is another route that presumably the company is doing or will have to do, is likely to have to do at some point?

Materials: Well on rotating parts where we need the higher temperature capability there is no substitute for it.

Environment: And that's a conscious thing to preserve rhenium for the uses where we need it presumably.

Materials: It is, yes, yes.

Environment: And the cost.

Materials: Well it's sustainability and cost, they're both key, cost in the short term, sustainability in the long term. 
Further discussions also highlighted the importance of social responsibility issues, focusing in particular on concerns associated with the supply of cobalt, highlighted in the following excerpt which includes the researcher, facilitator, Head of Supplier Engineering (supplier), Environmental Strategist - Civil Future Programmes (Strategist) and Global Commodity Leader - Raw Materials (materials):

$\begin{array}{ll}\text { Supplier: } & \text {..the other spin-off, major spin-off I think, will be the } \\ \text { corporate and social responsibility perspective so where } \\ \text { in our extended supply chain are there or may there be } \\ \text { practices which we would not want to be associated with } \\ \text { in the papers. } \\ \text { And indeed cobalt you have stopped using for that precise } \\ \text { reason in this country, which I think somebody has } \\ \text { mentioned. } \\ \text { Fes or is not used in other countries anyway I mean } \\ \text { cobalt, copper mines where cobalt comes from [sensitive } \\ \text { Supplier: } \\ \text { text removed] } \\ \text { Researcher: } \quad \text { what do you know about this? } \\ \text { Materials: } \\ \text { to go on to sort of the [sensitive text removed] so I thought } \\ \text { we were going down that road when that was mentioned } \\ \text { which is clearly a brand issue if something was obviously } \\ \text { you know produced from that area. I think it's valid but I } \\ \text { don't think it is just for cobalt, I think the problem is when } \\ \text { you are into the mining industry as a whole, that is } \\ \text { probably fairly widespread in most areas like South } \\ \text { America and Africa and now therefore is applicable to } \\ \text { probably all the materials that we spec on all our engines } \\ \text { across all the sectors, so that is potentially huge. } \\ \text { There aren't any equivalents of fair trade cobalt. }\end{array}$

Potential risks associated with energy use, from business operations specifically, produced an interesting result in the output charts. In the near to medium term it was not deemed to be a significant issue. However, in the long term it was considered a potentially very significant problem, in large part due to regulations that will encourage energy users to reduce carbon emissions. This is reflected in the $\mathrm{CO}_{2} /$ climate change and energy costs bubble that moves progressively from the bottom left to the top right of the chart in Figure 4.3. This point is highlighted in the following excerpt including the researcher (who is referring to the output chart) and Head of Environmental Management (environment):

Environment: Yes, it's a growing interest but I don't think it's really affecting business at the moment, we're just, we just pay the tax basically.

Researcher: So it's mainly kind of going a bit like that really.

Environment: Yes I think you know maybe your big issue in the longer term and again it's like it's longer than ten years it is security of supply isn't it you know so power outages and stuff like that, are you going to be, are you going to have a reliable power supply? Equally the government's relying 
on markets to sort it, there's no overarching policy for securing the supply per se, it's a market driven thing.

These concerns were certainly driven from an environmental perspective, highlighted by the following excerpt:

\begin{tabular}{|c|c|}
\hline nvironment: & $\begin{array}{l}\text {...it will start to influence on the longer term where you } \\
\text { decide to build for example...grid mix could become more } \\
\text { important in the future in terms of your carbon accounting, } \\
\text { so you've got to reduce your carbon footprint of the } \\
\text { company which is becoming more important... think at the co because it's about cost of } \\
\text { the moment it's not there becau l'm not aware } \\
\text { labour, where the customers are you know } \\
\text { of any factories being built at the moment on } \\
\text { environmental criteria otherwise we wouldn't be flattening } \\
\text { forests in Virginia, I mean you wouldn't be doing that } \\
\text { would you...I think it's a growing thing because it's a } \\
\text { growing interest, the government is requiring more and } \\
\text { more reporting in that area, it will become mandatory in } \\
\text { the UK in the next few years, customers like DOD in } \\
\text { America and MOD are getting very interested in it all, so. }\end{array}$ \\
\hline
\end{tabular}

Use phase $\mathrm{CO}_{2}$ regulation was deemed to be a very significant threat, albeit in the longer term, with potentially very serious consequences for the future of the business. Fuel efficiency in use has long been a primary requirement in the design of RollsRoyce's products. However, some participants expressed concern that regulations might be put in place stipulating specific performance requirements, in addition to current customer driven fuel efficiency targets. Participants felt that if the targets were not set realistically this could have significant implications for the business. The following excerpt highlights the concern, involving the researcher, Corporate Development Manager (development), Environmental Strategist - Civil Future Programmes (strategist) and Head of Environmental Management (environment):

Researcher:

Development: Strategist: Researcher: Strategist:

Environment:

Strategist:

Researcher: did you mention specific $\mathrm{CO}_{2}$ legislation, so it won't just be a commercial driver, it might actually be a regulatory requirement?

Well in terms of $\mathrm{CO}_{2} \ldots$

It could be a certification requirement. Is that a risk?

Yes especially in recent products the competitors tend to have better fuel burn so if they hadn't moved towards a target it's more aggressive than we can achieve.

I mean so there's lots of measurements, it sets specific limits and you have got to be above this you know.

Yes at the moment it's sort of driven by competitive market pressures to keep us in but if on top of that there's the potential for a more stringent requirement that is even harder and we obviously have a say to try and shape and inform it but there is a risk yes that we could be squeezed out of business because of...

...is that going to drive us to do anything we don't do at the moment? 


\begin{abstract}
Strategist: $\quad$ Yes, I think as I mentioned before it's going to poteintially anyway force us to invest more to stay in business. It might affect the focus about where do $R \& D$, indeed it might make us have some more difficult choices about which businesses we can sustain.

Researcher: Would you stick it on here? I mean if we were going to put it on here where would we put it?

Strategist: It's ten years, it's at the end, sort of beyond ten years I think, severity...[sensitive text removed].
\end{abstract}

The final comment by the strategist shows how potentially significant regulatory requirements for use phase efficiency could be. Whether or not this was within the scope of the focus group was questioned, as it is more of a strategic issue, rather than something that might affect tactical level design decisions. The relevance of strategic issues within the focus group outputs is discussed more in section 4.4.2.

Air quality, particularly how new standards could limit access to the market, was judged to be a moderately severe risk in the medium to long term. Air quality has been an important issue within the aviation industry for some time, driven by quality standards that have placed limits on emissions of nitrogen and sulphur oxides (NOx and SOx), particularly around airports during ground operations, take-off and landing. Improving impacts on air quality has presented difficult technological challenges. To improve fuel efficiency it is desirable for the engine to operate at higher temperatures, although a hotter engine will emit more NOx, requiring trade-offs between fuel efficiency and air quality. The group also felt that efforts to reduce impacts on air quality were likely to impact other sectors beyond aviation, which historically has been the main focus. These points are highlighted in the following excerpt, involving the Improvements Manager - Corporate HS\&E (improvements) and researcher:

$\begin{array}{ll}\text { Researcher: } & \text { And you talked about local air quality. } \\ \text { Improvements: } & \text { Yes it's an environmental regulation that will affect access } \\ & \text { to different markets or countries. } \\ \text { Researcher: } & \text { Is that for all our businesses or just the? } \\ \text { Improvements: } & \text { Yes I would say that will affect all businesses. I mean you } \\ & \text { know you've seen it with Concorde and noise and where } \\ & \text { you couldn't fly Concorde...trying to put energy products } \\ & \text { into California with the air quality limits there you know it's } \\ & \text { extremely difficult because you've already got stringent air } \\ & \text { quality so I can see it applying to all sectors again and I } \\ & \text { think the marine you could argue that there may be limits } \\ & \text { that could affect what ports you are allowed to go into and } \\ & \text { things. } \\ \text { Because air quality is on the...next environmental action } \\ \text { programme as to what the priorities are, some of the air } \\ \text { quality is in that. }\end{array}$ 
The following excerpt involving the Environmental Strategist - Civil Future Programmes (strategist) and facilitator suggested that in the future fuel efficiency will be a more important requirement than air quality:

$\begin{array}{ll}\text { Strategist: } & \text {... legislative environment for } \mathrm{CO}_{2} . . . \text { a new standard or } \\ & \text { let's say even if it becomes a certification requirement to } \\ & \text { meet a standard, so once you set in place a means of } \\ & \text { measuring it, it might be more than an aspirational goal } \\ & \text { for people, it might be our next product might actually } \\ & \text { have to meet it. } \\ \text { Facilitator: } & \text { Yes. } \\ \text { Strategist: } & \text { Which is a lot more worrying than potentially the noise or } \\ & \text { the NOx sort of thing, it's going to drive technology } \\ & \text { whereas before it has only been market driven. }\end{array}$

The final hazard placed on the output chart was global producer responsibility legislation, which was judged to represent a moderate threat in the medium to long term. Participants could see a lot happening relevant to other industries and it was felt that sooner or later these types of initiatives would begin to affect the aerospace industry. This point is highlighted in the following excerpt also involving the Improvements Manager - Corporate HS\&E (improvements) and researcher:

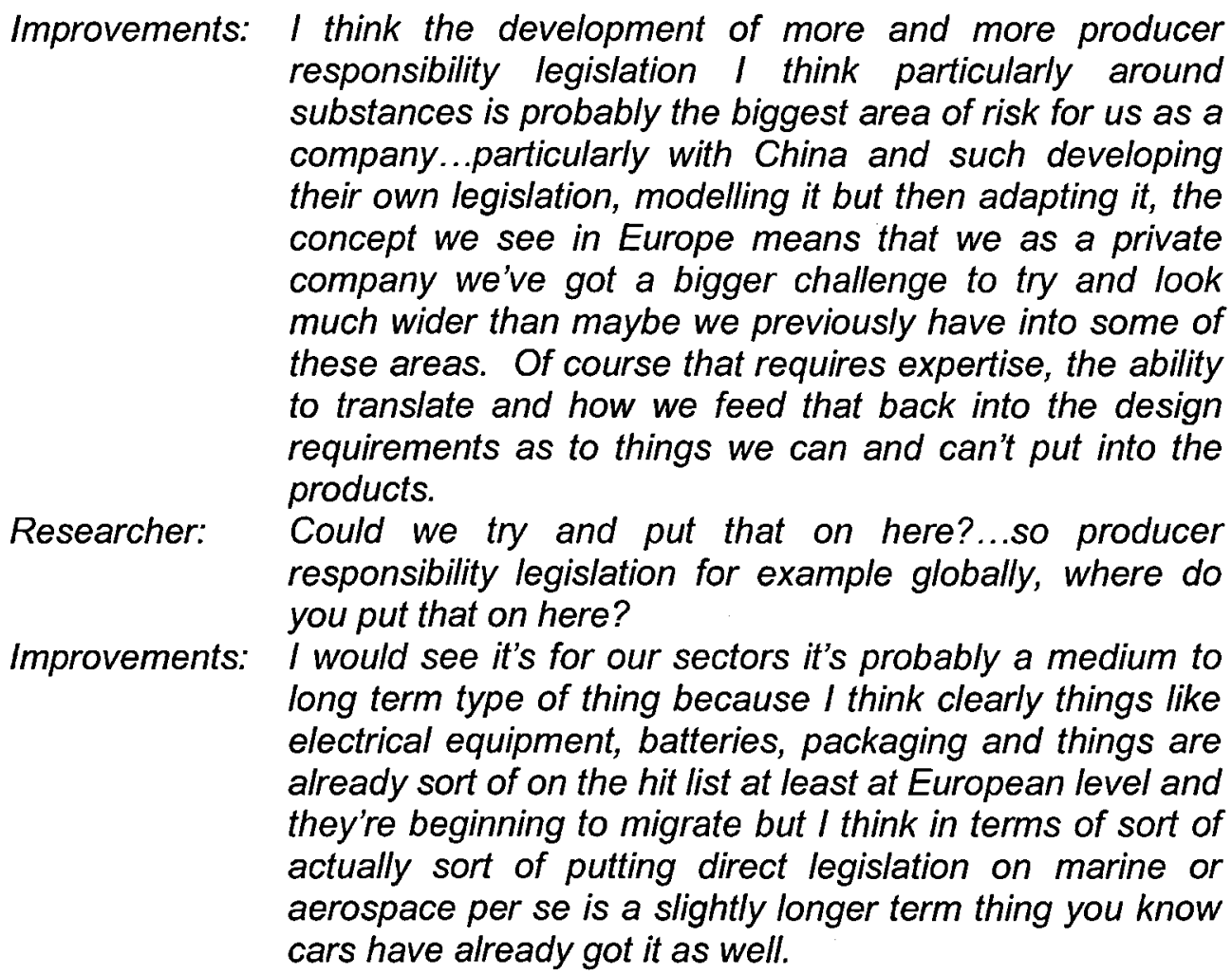

Summarising the results from focus group one, $\mathrm{REACH}$ and access to resources were concluded to be the most important hazards in the short term, given their position towards the top left hand corner of the chart in Figure 4.3. Hazards that were likely to be significant in the future included use phase $\mathrm{CO}_{2}$ regulation, energy costs and 
security of supply, regulations to improve air quality affecting access to markets and global producer responsibility legislation.

\subsubsection{Focus group two}

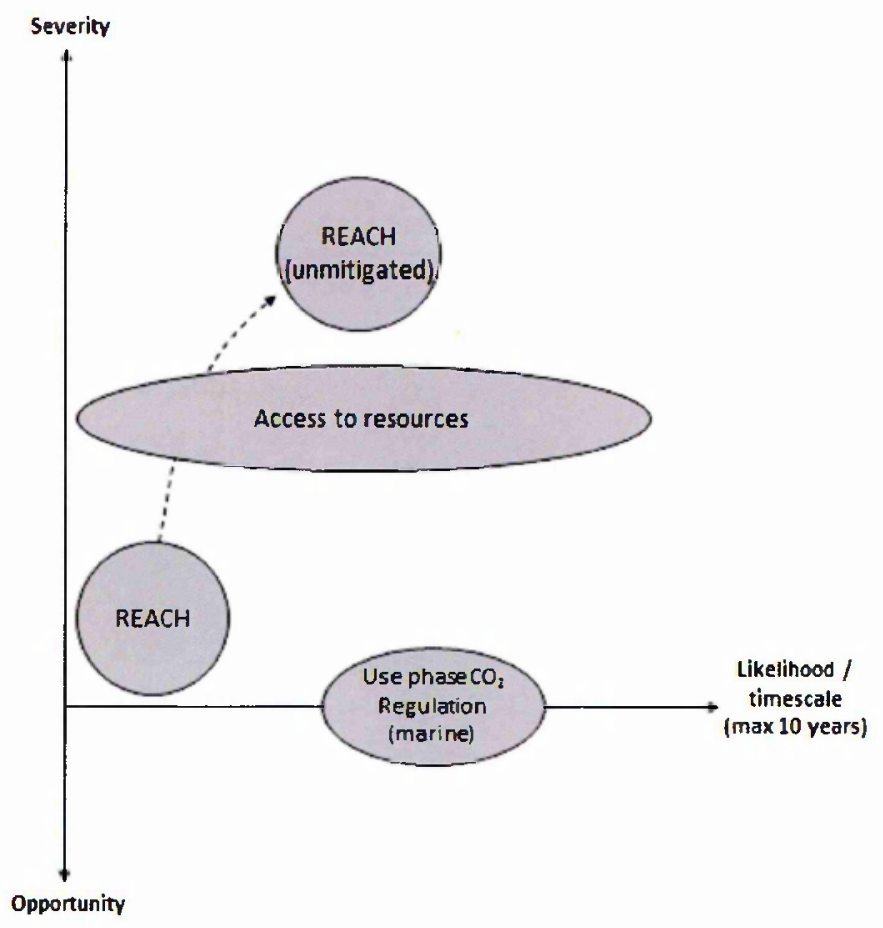

Figure 4.4: Output chart from focus group two

Figure 4.4 displays the output chart from the second focus group. Participants in the second group picked out the following hazards for the screening exercise:

- REACH (use of hazardous substances) (timescale);

- Access to resources (strategic alloying elements) (timescale);

- Use phase $\mathrm{CO}_{2}$ regulation, particularly for the marine business (likelihood).

In general the second group found selecting and prioritising hazards a more challenging task, reflected in fewer hazards being placed on the output charts. REACH was discussed at length throughout large parts of the focus group, participants offering argument and counter-argument as to whether or not it was a significant threat to the business. Opening discussions on REACH highlighted how it was seen as potentially less significant, captured in the following excerpt involving the Head of Manufacturing (manufacturing) and Engineering Purchasing Executive (purchasing):

Manufacturing: Well REACH is becoming less of an unknown isn't it ...we've understood for a long time which are the harmful substances that we were trying to drive out of our product before REACH ever came along...like asbestos...and 


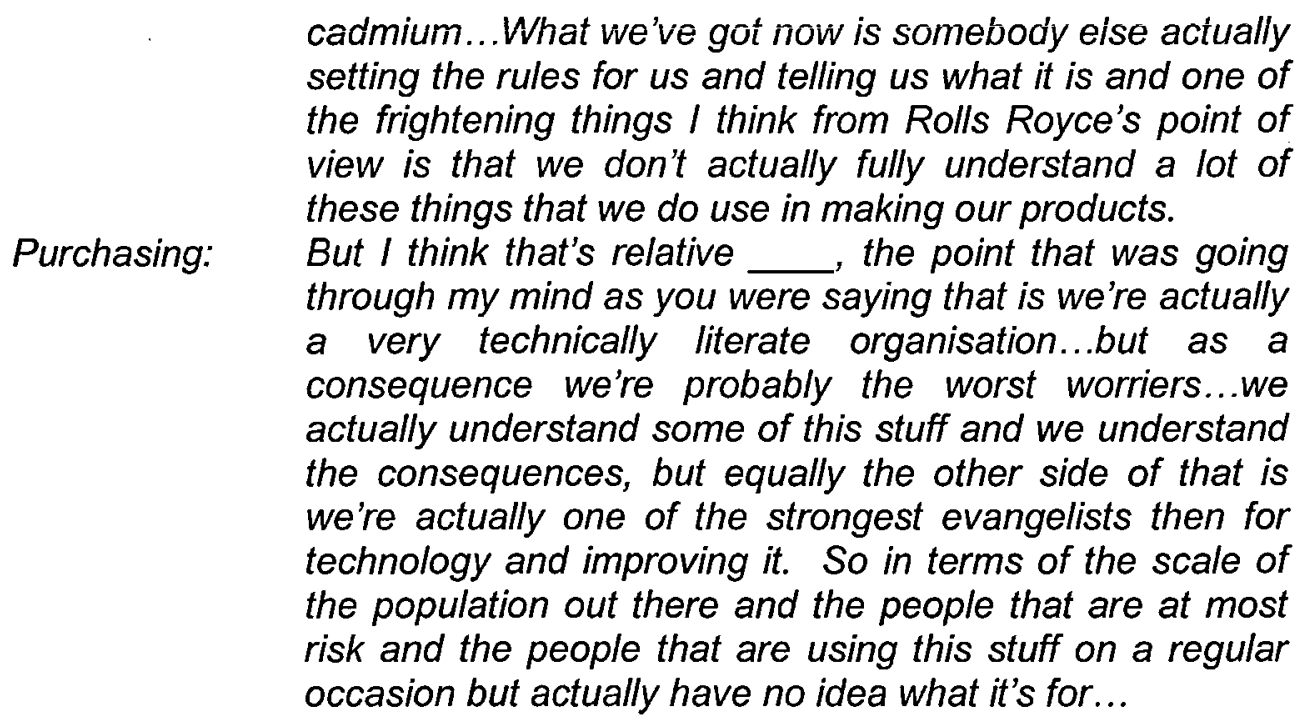

The following excerpt highlighted how other participants were more concerned, including the Materials Specialist - Corporate HS\&E (specialist):

Specialist: $\quad$...There's REACH of course which is changing the whole dynamics of the arguments because stuff which you think should be perfectly reasonable for us to use is suddenly being hammered with an obsolescence threat that you don't really like.

REACH is a complex piece of regulation, which can affect the business in different ways. Participants struggled with placing REACH on the output chart, as they felt that different parts of the regulation should be considered separately. This point is highlighted by the following excerpt, which also involves the Capability Lead - Life Cycle Engineering (capability):

Purchasing: Well no but in the context of this then I mean your question is where would I put REACH.

Specialist: I think you would put each of the different bits in different places.

Purchasing: Where I would put REACH based on the actions that we are taking, I mean the statement that made was we don't foresee any significant delay but that's based on the actions that we are taking. Now you know are there bound to be things popping out of the woodwork that catch us by surprise?

Specialist: $\quad$ Yes.

Purchasing: Yes, are there stuff, is there stuff that pops out of the woodwork today irrespective of REACH to catch us by surprise? Yes, all the time. So is REACH any different? Only in its magnitude in terms of affecting these things and it's just going to affect a whole bunch more stuff.

Manufacturing: It's in the bottom, to me it's in the bottom quarter easily.

Capability: It's the first time you've got regulation which is now really starting to drive substances off the market it will grow, in our lifetime it will grow... and for the first time you won't have any choice. 
Some participants felt that REACH was less significant as the business would focus resources to solve any significant problems if necessary. However, the lack of money being put towards developing alternatives for restricted substances was highlighted as a potentially significant issue, highlighted in the following excerpt which also involves the Technology Strategy Manager (technology):

Manufacturing: You've got to look at what the real risk is because the fact that you've got a hex chrome on something is because it corrodes so I can give you a material that doesn't corrode, it's going to cost a lot more and you might have to redesign all sorts of bits and pieces but it's not beyond the wit of man.

Specialist: $\quad$ And because you can do that you are not going to get given an authorisation to allow you to carry on using hex chrome.

Technology: That scares me because I mean the technological selection process won't be funding R\&T, I'm not aware, I might be wrong, that there's been any submissions last year or this year to say we want to submit this research in order to replace

Specialist: $\quad$ There was and it was turned down.

Manufacturing: It was, it was and it was thrown out each time, it's been thrown out consistently.

Technology: I wasn't here that time.

Specialist: It's really starting to annoy us now.

Purchasing: Which is why, which is why the response to the FT article was great, we might get some attention now.

(The Engineering Purchasing Executive was referring to an article covering RollsRoyce's concerns over REACH published in The Financial Times (Marsh, 2010), two weeks before the focus groups were run).

Participants found it worrying that a judgement could not be made on the significance of REACH, although ultimately it was deemed to be best judged as unmitigated, representing a moderate to high threat in the medium term, as highlighted in the following excerpt also involving the researcher:

\begin{tabular}{|c|c|}
\hline Researcher: & $\begin{array}{l}\text { I mean we said at the beginning we didn't want to turn this } \\
\text { in to a REACH talking shop. }\end{array}$ \\
\hline Manufacturing: & $\begin{array}{l}\text { It's just a bit worrying isn't it that we can't put REACH, we } \\
\text { can't agree where REACH fits on that chart... }\end{array}$ \\
\hline urchas & $\begin{array}{l}\text { If you want, going back to logic, if you want to do this } \\
\text { unmitigated in other words where are we right now and if } \\
\text { we all stop doing what we are doing then you can put it } \\
\text { fairly hard towards the top right-hand side. }\end{array}$ \\
\hline
\end{tabular}

This final result is shown in the output chart for focus group two shown in Figure 4.4, with REACH initially being placed in the bottom left of the chart, moving towards the top right. 
Concerns over access to resources were raised on at least two occasions during initial group discussions, with a particular focus on restrictions in the supply of rare earth elements, highlighted by the following excerpt involving Materials Specialist Corporate HS\&E (specialist):

Specialist: $\quad$...all of our products are long lifecycle product, all of them and therefore we have reason to worry...the ones that worry me more are resource use and scarcity of supply and obsolescence threat of materials and actually being able to make a product and that can be brought about by a whole host of different things...It can be because certain rare earth materials...China has said that they're going to stop selling them to the rest of the world in 2012 which kind of matters...

Later on the group discussed the issue in more detail, considering substitution, metal usage rates and impacts on cost, as highlighted by the following excerpt, also involving the Capability Lead - Life Cycle Engineering (capability), Engineering Purchasing Executive (purchasing), Head of Manufacturing (manufacturing) and Technology Strategy Manager (technology):

\begin{tabular}{|c|c|}
\hline Technology: & $\begin{array}{l}\text { We don't know what the price is going to do but the risk is } \\
\text { understood so it's just a question of as you say are you } \\
\text { going to mitigate it technically. }\end{array}$ \\
\hline Specialist: & $\begin{array}{l}\text { But it's just I can't see how you are going to get it at all } \\
\text { because if it's only produced in China and China have } \\
\text { said they are going to stop selling it, what do you do? }\end{array}$ \\
\hline $\begin{array}{l}\text { Technology: } \\
\text { Manufacturing: }\end{array}$ & $\begin{array}{l}\text { Yes but you have lower, you have worse SFC. } \\
\text { In fact we're actually already doing those based on cost } \\
\text { because they are so expensive... That's because they're } \\
\text { rare...Because we're actually we're backing off on a } \\
\text { number of these things and going back to first generation } \\
\text { and using those instead and making other adjustments. }\end{array}$ \\
\hline & Irrespective of what we've said. \\
\hline $\begin{array}{l}\text { Capability: } \\
\text { Manufacturing: }\end{array}$ & $\begin{array}{l}\text { Exactly, yes. } \\
\text { I mean it's, the telly programme was interesting because } \\
\text { they showed the XWB fan case and they said it is, it's the } \\
\text { biggest we've made, five metric tons of metal to start with } \\
\text { and it ends up at five hundred kilos and I'm thinking } \\
\text { Christ, I wish you hadn't have said that. }\end{array}$ \\
\hline tapan & \\
\hline
\end{tabular}

(The Head of Manufacturing was referring to a recent television documentary on how to manufacture a jet engine).

It was also discussed how mitigating risks from possible resource restrictions would be easier for new designs than existing products, as highlighted by the following excerpt:

Specialist: $\quad$ There are two actual issues with rare earths. One is the, what we can do in new design which is easier to mitigate ...And the second is where we've already incorporated it 
into the design that we're flogging, I mean you have to keep on getting hold of it to be able to service that and replace those parts, that's a different order of magnitude, that's worse because you have to then keep on trying to get hold of it even if the world supply gets to be really very, very [at this point the facilitator jumped in]

As with REACH, participants genuinely struggled to make a judgement on where access to resource should sit on the output chart. It was considered to be potentially very significant and both a current problem and a problem into the future, represented by the elongated bubble across the middle of Figure 4.4 .

Carbon emissions were the first topic raised by the group, initial discussions suggesting that a primary concern for the business was being perceived as a large carbon emitter. Although it was thought that the marine business was currently not as affected as aerospace. This point is highlighted by the following excerpt, which also involves the Executive Vice President - Civil Nuclear (nuclear):

Technology: $\quad$...So from a business sense the continuing change in greenhouse gas legislation, in the emissions legislations and the impact therefore on what our products look like that to my mind is the biggest impact on the business in terms of what it is we sell and on what shape and form that should take.

Facilitator: $\quad$ So the performance level and associated acceptability of the products.

Technology: Yes, yes.

Facilitator: OK.

Manufacturing: I think that's one of the biggest issues because it's the perception of the company. I mean if you talk to your average punter they think that jet engines generate more greenhouse gases than anything else in the world and you know we constantly fight to overcome that. So you know from the actual product, product uses there's that. I mean you know nuclear reactors and things they're pretty dodgy as well aren't they.

[Laughter]

Nuclear:

Oh I don't know!

Purchasing:

We've got it on both sides haven't we, I mean sort of we're becoming a fossil fuel pariah if you like, that that discussion is quite prevalent in the aerospace industry right now. Interestingly with the marine I don't think that debate has particularly started yet so I think we are under the radar when it comes to marine.

However further discussions highlighted how environmental issues were becoming important in the marine sector, as shown in the following excerpt involving the Head of Research and Technology - Marine (marine):

Marine: Well the interesting things we're starting to see in the shipping industry is there are a few customers who are now starting to basically take on a policy of green 
operation even though it costs them more. Belenius Wilhelmson the Scandinavian operator that operates oil and gas carriers and car carriers around the world they actually took a decision to use more refined diesel fuel which doesn't have the sulphur in it that most of the major operators around the world use, in fact they actually have to ship this fuel to some areas of the world so that they can bunker it into their own ships but in port they don't emit you know the big black clouds of sulphurous smoke and they're doing lots of other things as well to show that their ships are you know particularly green and they are

Facilitator: So it's not cost effective in a conventional sense but they are taking the hit for other reasons.

Marine: It's nearly twice, it costs you nearly twice as much money for the fuel basically but one of the interesting side effects of that is that Toyota when they were placing the world contract for shipping their thousands of cars around the world because at any point in time there are tens of thousands of Toyotas on the high seas being moved around the world from manufacturing to end use, point of sale, Toyota thought this was great that they can actually go with Belenius because they were operating very much as a green operator and it was good for Toyota to say you know we move our cars around the world in the most green and environmentally friendly way possible in our partnership with Belenius.

Technology: And presumably Toyota paid more for the pleasure against somebody else?

Marine: I think so yes I mean but because of the sheer scale of what they are doing and you know obviously

Facilitator: It's a pointer for the future though.

Marine: $\quad$ But I think it's interesting and we've seen one or two ferry operators doing that as well.

Further discussions focused on potential new $\mathrm{CO}_{2}$ emissions regulations in the marine industry, similar to the use phase $\mathrm{CO}_{2}$ regulations for aerospace discussed in the previous focus group, as highlighted in the following excerpt:

Marine:

Yes I mean it already is doing because there is actually proposed legislation from IMO [International Maritime Organisation] coming through in terms of controlling the $\mathrm{CO}_{2}$ output of ships which will actually leapfrog aerospace because they will actually be directly controlling $\mathrm{CO}_{2}$ output which they don't, as I understand it, they don't do in aerospace yet or there's no legislation planned.

Technology: It's not an accumulative what the industry is missing in a sense.

Marine: $\quad$ No, no.

Facilitator: What is the timescale on that? Is that something that's been sprung on the industry so to speak or is it something that's been creeping up for a while?...

Marine: $\quad$ Well talking to one of the guys from IMO a few weeks ago he was saying it could come in as early as 2014 or 2015. 
However as discussions progressed the group concluded that $\mathrm{CO}_{2}$ emissions regulations in the marine industry could represent a business opportunity as well as a threat, and should be captured as so, hence the position of the relevant bubble in Figure 4.4, as highlighted in the following excerpt:

$\begin{array}{ll}\text { Nuclear: } & \text { Fundamentally, does it give a competitive disadvantage or } \\ & \text { a competitive advantage? Is it a disadvantage to our } \\ & \text { product? } \\ \text { Marine: } & \text { Actually I think } \\ \text { Technology: } & \text { How about back to the issue of what severity is. } \\ \text { Marine: } & \text { I think it will actually...enhance our position...I think it will } \\ & \text { enhance our position so. } \\ \text { Nuclear: } & \text { So it's not a business risk in that sense at all. }\end{array}$

On the whole the second group found it a lot harder to pass judgements, often acknowledging how difficult and complex a lot of the problems being discussed were, as the Head of Research and Technology - Marine commented: "you can't just say well this is all too difficult". These difficulties are discussed more in the next section. Overall, from the output charts, the second group agreed with the first in that REACH and access to resources were the most significant threats, although they did disagree on how severe they might be and over what time period.

\subsubsection{Results summary}

Table 4.5 provides a summary of results, listing the top five hazards from the keyphrase analysis and hazards that were assessed using the output charts from focus groups one and two.

Table 4.5: Summary of results

\begin{tabular}{|c|ll|}
\hline $\begin{array}{c}\text { Key-phrase } \\
\text { analysis }\end{array}$ & REACH & $(14)$ \\
& Access to resources & $(8)$ \\
& $\mathrm{CO}_{2}$ /climate change and energy costs & $(6)$ \\
& Brand and reputation & $(6)$ \\
& Use phase $\mathrm{CO}_{2}$ regulation & $(6)$ \\
\hline $\begin{array}{c}\text { Output chart: Focus } \\
\text { group one }\end{array}$ & Immediate threats: \\
& REACH \\
& Access to resources \\
& Longer term concerns: \\
& Use phase CO 2 regulation \\
& Air quality (access to markets) \\
& CO $/$ /climate change and energy costs \\
& Global producer responsibility legislation \\
\hline $\begin{array}{c}\text { Output chart: Focus } \\
\text { group two }\end{array}$ & REACH \\
& Access to resources \\
& Marine use phase $\mathrm{CO}_{2}$ regulation (also recorded \\
& as an opportunity) \\
\hline
\end{tabular}




\subsection{Discussion}

The focus groups provided useful insights into how environmental problems are perceived within Rolls-Royce and a picture of what focus group participants thought were the main threats to the business. To draw out conclusions from the results, a discussion of the focus groups is presented in the following sections, covering the following topics:

- The focus group results, in terms of the hazards identified and how important they were deemed to be;

- General perspectives on the problem of identifying hazards, including the scope of the exercise, which was a contentious issue within the second group in particular;

- How the focus groups worked and how lessons learned from the exercise may influence the approaches used to identify hazards in the future.

\subsubsection{Focus group results}

REACH and access to resources stand out as the most prominent hazards from the summary of results provided in Table 4.5. REACH was the most frequently discussed topic, dominating discussions in the second group in particular. Access to resources was also prominent during discussions in both focus groups. Both hazards also featured in the output charts and were judged to be significant threats considering the severity of the potential impact and when (or how likely) these impacts were to occur. Combining the results of the key-phrase analysis and output charts, the results show that REACH and access to resources are environmental business hazards of valid concern to Rolls-Royce.

REACH is a key concern largely due to the business risks posed by substances becoming unavailable due to authorisation and focus group one highlighted particular risks related to the use of hexavalent chromium compounds. Making engineering changes to cease the use of these materials is likely to be a significant undertaking, which was confirmed through separate discussions with engineering fellows after the focus groups. Investment in developing alternatives is also likely to be costly and time consuming, which was also emphasised through separate discussions.

Access to resources was seen as a significant problem now and in the future. Short term concerns focused on cost in particular, although both groups acknowledged how a combination of socio-economic factors can lead to supply disruptions, posing a significant business risk. Supply monopolies (using the example of China and rare 
earth elements) were highlighted as a concern, as was the supply of rhenium largely due to its production as a co-product, making supply inelastic to changes in demand. In the longer term sustainability considerations were seen as important by the first group in particular, including the need to address potential social responsibility issues in material production supply chains. The supply of cobalt was highlighted as an example where social responsibility considerations have been important.

It was surprising that environmental business hazards related to $\mathrm{CO}_{2}$ emissions and climate change were not the most frequently discussed topic, given the nature of the products made by Rolls-Royce. Related discussions on energy and use phase $\mathrm{CO}_{2}$ regulations did figure prominently and the second group certainly felt that being perceived as a "fossil fuel pariah" (as one participant commented) was a significant business risk. However, climate change is also quite a strategic issue, and hence possibly outside of the scope of the tactical focus required within the groups. Climate change is also a well known issue and the business already invests significant amounts of money on research and development to mitigate these impacts. Another explanation is that the groups felt it would be better to focus on less popular topics. These factors might explain why climate changes discussions did not figure more prominently and this point is discussed more in the next section.

It was difficult to identify a tangible impact on the business from most of the hazards identified within the focus groups, which was a surprise. Hazards such as sustainable procurement and broader global producer responsibility legislation were highlighted as being important. However, there was nothing discussed about these hazards that pointed to a direct impact on a product design or requirements, which is because these issues are not currently applied to aerospace products, although the fear is that they might be. Understanding how these policy instruments have affected other industries may indicate what the potential impact on the aerospace industry might be.

Sustainable procurement and global producer responsibility regulations are likely to have an impact further into the future and it is not possible to identify a tangible effect at this time. It was expected that hazards related to specific environmental management issues within operations might come out of the focus groups. These hazards might impact on for example, the ability to make a product or product cost, however such topics were not really discussed. Possible reasons for this are also discussed in the next section.

Reputation and brand image, and related effects such as the ability of the business to recruit and maintain a high-skilled work force, were also highlighted as very important hazards. Within the second group, the communications representative (comms) 
provided an interesting insight into Rolls-Royce's approach to managing the reputation of the business in an environmental context, following a thread discussing lobbying activities related to REACH:

Environment: $\quad$... I mean we were approached by some people to join a lobby against REACH but it's very difficult to lobby against something when the concept of it is to promote human health and the environment...you can't come out and say no we don't support that but what you can do is come out and say well yes we in principle we think it's a great idea but we have some concerns about how that will impact...for whatever reason so that was the tack that we took.

Facilitator:

is there anything you'd like to add since we are getting nearer to your neck of the woods at the moment?

Comms: ...you can look at each issue individually but you can also look at an issue as a whole and if we put our heads and we respond very vociferously on one issue then we also put our heads above the parapet for other issues in regards to the environmental context. So I think just to you know say why didn't we defend ourselves on this issue well you know responses were made, background briefings took place you know a lot of work did go on from you know within the communications, just because you don't always see it doesn't mean that the work hasn't taken place. So l'm just saying sometimes it's not a case of straightforward standing up there and defending a position that's all, I'm just saying there are other options and sometimes a longer view strategy on certain issues that's all. I know it sometimes maybe feels like nothing has been responded to but that's not always the case.

Purchasing: I think__ that's fair, I think you know we need to pick our battles and I think if you take a positive one I mean the impact that Sir John Rose had, has had on the perception of industry within the UK in manufacturing in the last two years is astonishing... but I think there is again picking up on __ point you know I don't think we try to identify ourselves within Rolls Royce with this subject, I think we just try to toe a very straight you know conservative line on this and not rock the boat too much.

(Sir John Rose was the Chief Executive of Rolls-Royce at the time of the focus groups).

Discussion also included concerns about Rolls-Royce's environmental image affecting the company's ability to recruit. This point is highlighted in the following excerpt from the second focus group:

Nuclear: $\quad$...when I look at my kids they are massively more environmentally aware than I ever was at school. It seems to me that as time goes forward companies are going to have to be explicitly environmental, it's not in my creed in the same way, I think it'll actually become a skill enabler if you like. 
Specialist: Yes in fact that's a whole different thing, the lack of availability of suitable brains coming out of university but that's not really environmental is it.

Nuclear: $\quad$ No but it's a bizarre one isn't it because what it's saying is Technology: $\quad$ You won't get them.

Nuclear: $\quad$ You won't get people...it's a business risk.

Technology: If aviation is dirty and Rolls Royce is what makes aviation engines QED Rolls Royce is dirty regardless of the facts...

Facilitator: It is a side issue though it's certainly part of the mix as a whole, is it already true? You find recruitment a problem now?

Specialist: $\quad$ Yes it is.

Purchasing: I can give you specific examples where graduates have said that they want to work for something that they think is value adding to society and that's why they didn't bother applying to Rolls Royce.

In the first focus group the Head of Environmental Management picked up on specific reputational issues surrounding a new piece of regulation in the UK, which seeks to reduce energy use from businesses, as highlighted in the following excerpt:

Environment:
pothere's regulation now with indexes that will in effect can
performing rather less well than you really are... So in
terms of brand and reputation the company could get
tarnished and the board is worried about this, not the fact
that there's something called the carbon reduction
commitment regulations and it's going to cost us say
another million pounds a year in tax, the board's not
worried about a million quid in tax, they'll just pay it but
they are worried about where we appear in the league
table and because of what we do we could go to the
bottom that will affect brand, the reputation.

Despite reputational hazards being highlighted as important, nothing specific was identified that could take these concerns on to the next step to better understand the business risk.

As discussed at the beginning of this chapter, the main purpose of identifying hazards was to produce a list of hazards that have been shown to be of concern to Rolls-Royce and that can be used to test the remainder of the framework for environmental risk management. Discussing the results it is clear that the REACH and access to resources hazards are of concern to Rolls-Royce and these hazards are also sufficiently tangible to be used to assess the risks posed to a Rolls-Royce product design.

Whilst not the biggest topic of discussion, hazards related to $\mathrm{CO}_{2}$ emissions and climate change did figure prominently in focus group discussions, being ranked third in overall discussions. The position of the energy use bubble in the output chart from 
focus group one (Figure 4.3) also suggested it was a serious concern in the longer term. To include a hazard to reflect concerns about $\mathrm{CO}_{2}$ emissions, the $\mathrm{CO}_{2}$ /climate change and energy costs hazard is also selected to test the framework. It is clear how this hazard can be used to assess the risks posed to a Rolls-Royce product design, as increases in energy costs to manufacture parts could impact on design cost objectives. Longer term concerns focused on whether increases in energy prices may have a significant impact on the business and it would be interesting to analyse how energy prices may impact on product costs.

\subsubsection{The problem of identifying environmental business hazards}

Discussions within the focus groups provided some useful insights into the broader problem of identifying hazards, focusing on three areas:

- What the problem actually was, including what was meant by the term 'environmental business hazard';

- The scope of the exercise, in particular the difference between strategic and tactical issues;

- Perspectives on what the company needed to do to identify environmental business hazards in the future, including the time period over which environmental business hazards might be identified.

Discussions suggested that participants broadly understood the problem of identifying environmental business hazards, even if different language was used, as highlighted by the following excerpt from focus group two:

Purchasing: $\quad$...what ultimately $I$ assume what you want to drive at at the end is what are the prime drivers for Rolls Royce in terms of designing for the environment...

The Engineering Purchasing executive also noted existing company activities that broadly sought to achieve the same goal, in terms of influencing the design process regarding particular problems:

Purchasing: Yes I think that's the whole principle of what we're trying to do is through the governance of substance and the Material Stewardship Board, I mean it's exactly that sort of logic that says take all the various different pressures and legislation and the one that keeps coming up is REACH obviously but it shouldn't be, it should be any type of pressure that says what's the policy on our new designs from now on and for that matter in our factories today... 
The following excerpt also emphasises the importance of understanding how risks impact on product designs:

Purchasing: Well my greater concern actually is we have a level of technology in our product that the risk of producing nonconformance somehow is actually the one that probably concerns me more than anything else...[other problems are] a secondary consequence to any of the risks that flow through to our product.

Participants also definitely acknowledged the need for a forward looking view regarding identifying environmental business hazards, adopting a pro-active approach to future threats, as highlighted in the following excerpt also taken from the second focus group:

$\begin{array}{ll}\text { Specialist: } & \text { But the point is is that if we can spot those things } \\ \text { sufficiently in advance then we can start to curve the ball } \\ \text { well in advance. With REACH if it wasn't for we } \\ \text { wouldn't have been able to do anything, we wouldn't have } \\ \text { done anything on that, we would have been completely } \\ \text { blindsided by it but }\end{array}$

Similar comments were made by the Corporate Development Manager in the first focus group:

Development: It feels like there should be a better pipeline of risk in the way we manage things that perhaps we might see it come up on the legislative environment but the way it's played through and then monitored on an ongoing basis doesn't then allow us to sort of categorise and update that risk as it sort of comes towards the mid term.

These comments show that problem of identifying hazards, as it was described at the beginning of this chapter, is a valid and important one for the business to continue to address. Within the first group there was some discussion on what actually was meant by the term 'environmental business hazard'. Participants acknowledged that any definition is going to be difficult, as the Head of Supplier Engineering commented: "to some extent a definition of environmental business hazard is going to be woolly and ambiguous...". However it was agreed that whatever definition was used it should include the phrase 'potential to cause harm'.

Setting the scope of the exercise presented a particular problem prior to the focus groups. Participants requested some guidance on what was or what was not relevant to the topic. However, providing guidance on the scope would have effectively 
compromised the outcomes of the groups as being representative of a Roils-Royce view, so the initial scope of the discussions was left deliberately broad. One of the biggest issues was whether or not the exercise focused on, broadly speaking, any type of hazard that could impact the business, or had a specific focus on tactical issues, such as REACH. Focusing on the topic of Design for Environment more broadly, this point is highlighted in the following excerpts from the second focus group:

Technology: Your phrase design for the environment I struggle with because it splits into two elements, what you design is part one...or there's what is it I am allowed to use in designing something which comes back to the point is it something the designers should be worried about that, it should be something yes...

Specialist: $\quad$...there's lots of different things at different levels isn't there. At the overall strategic level of the company the outcomes could just direct us down a specific thought process like are we going to invest in tidal turbines and then on the concept design it could say well this particular concept design is going to be without certain metals in it, and it's going to be chromate free and that's a decision you can make right upfront at the beginning of a new engine process...

Discussions within the first focus group suggested that all issues were potentially within scope, even suggesting that the remit be expanded to cover 'design for sustainability', as highlighted in the following excerpt involving the Head of HS\&E Marine:

HS\&E Marine: Perhaps we have as a company been thinking too narrowly on this and are caught in the history of how this was viewed in industry ten, fifteen years ago. Perhaps it's a new day on the background of what all has been said around the table. Perhaps it is timely to start widening our concept field when we talk about design for something, perhaps design for sustainable development is a better concept, just a thought.

Facilitator: I can tell you there are a number of heads nodding around the table as you say that...

This discussion is useful as these comments can be used to set the scope of future activities that seek to identify environmental business hazards. Discussions would suggest that there certainly needs to be some activity within the business to broadly identify hazards from a sustainability perspective at tactical and strategic levels.

Bearing this in mind, other discussions within the focus groups provided insights into how the problem of identifying environmental business hazards could be approached in future. In the first focus group the Corporate Development Manager suggested breaking down the discussion to enable a better focus on specific issues, or phases of the life cycle, as highlighted in the following excerpt: 
Development: Personally l'd like to maybe see a bit more breakdown into the...phases between lifecycle. At the moment we are talking across all the phases at the same time which probably damages our conversation. If we have to start to focus on those basis because I don't feel l've contributed to the manufacturing phase very much but sort of you maybe have a follow up session to focus on those specific phases in more detail and then you might collect together more experts from the manufacturing community, more experts from the sort of operational community and just get a broader perspective on that because we haven't generated that many ideas and I'm sure there are a lot more ideas out there which should be pulled in.

The lack of a specific focus on particular life cycle phases might explain why more hazards were not identified from operational environmental management issues. In the second focus group the Executive Vice President - Civil Nuclear also highlighted the need for participants with a greater knowledge of the problem:

Nuclear: $\quad$ I think there's this point, if you listen to the debate we've not got the kind of maturity and understanding that enables it to be rolled out to a broader population without getting complete anarchy.

This excerpt possibly supports the argument for not running too many focus groups, instead picking a representative set of participants rather than attempting to cover all viewpoints in detail.

Views on the time period of which environmental business hazards might be identified were an important outcome from the focus groups. Participants acknowledged that there was a requirement for a forward looking view, but also that looking too far into the future was difficult, as highlighted by the following excerpt from focus group one:

Improvements: I suppose the difficulty is predicting the legislative landscape around the time that our products...come to end of life as well, you are looking at thirty year product life, what's acceptable now may not be acceptable in thirty years.

As a business Rolls-Royce has a planning horizon of ten years. It was suggested that perhaps this was the longest time period over which environmental business hazards could be identified, which was broadly agreed within both focus groups. Beyond ten years is also when scenarios are typically used to think about the future, which supports the use of alternative methods up to this time horizon. 


\subsubsection{Using focus groups to identify hazard's}

Another outcome from completing the focus groups was perspectives on whether or not focus groups were a suitable tool for addressing this type of problem. The focus groups were successful in so far as hazards were identified that could be taken forward to develop and test the framework for environmental risk management. The first group took to the task exceedingly well. All participants engaged in a lively debate for the first hour and were happy to make judgements on the significance of the hazards within the second half of the group. Participants saw value in the exercise and encouraged a broader remit and the running of more groups.

The second group did not work very well in comparison to the first. Discussions started well, although problems started to arise when it came to drawing judgements on the significance of particular hazards. There seemed to be more of a focus on the question being asked rather than answering the question itself. Participants really struggled with making judgements on the hazards that were deemed to be the most important, almost to the point of being fearful of being drawn into conclusions on how big a risk some of the issues might be. Participants also struggled generally with the scope of the activity, finding the problem rather nebulous and difficult to grasp.

It is felt that discussions in the second group broke down for two reasons:

1. There was not sufficient balance between senior managers and environmental specialists. Focus group one had an even mix of environmental specialists and managers and participants came across as being much more comfortable discussing environmental issues and their importance.

2. Whilst good for consensus forming exercises, focus groups are not ideally suited for decision making, and this was what was really required to judge the significance of hazards.

The first point suggests that problems in the second group were largely due to the participants present, rather than broader problems regarding the validity of the exercise. It was attempted to ensure an even spread of participants across both of the groups, although on reflection the second group did contain too many managers who did not have sufficient knowledge of the topics being discussed. Involving quite a few senior people also made it difficult to draw judgements on the significant hazards. This point is reflected in comments made by the Technology Strategy Manager during informal discussions at the end of the second group, who observed: "it was an interesting and valuable exercise, although you are going to struggle to get agreement in a room of people like this". The requirement for participants with greater specialist 
knowledge is also supported by previous excerpts from the first and second groups, which suggested the need for a more focused discussion.

Comments within the second group also suggested that this was not an appropriate forum for making decisions regarding how important environmental business hazards might be, as illustrated by the following excerpt:

\section{Purchasing: $\quad$...Rolls Royce wants to adopt this sort of thing for design ...it's got to be something which is governed through jurisdiction board, into policy...}

These observations suggest that not only should a more focused approach be taken to identifying hazards, involving people with a better knowledge of the topics being discussed, but also that the decision making part of the process should be conducted as a separate activity.

\subsection{Conclusions}

The objective of this chapter was to identify hazards that have been shown to be of concern to Rolls-Royce, which can be used to test the framework for environmental risk management through further research. Undertaking research activities to identify hazards has also provided insights into how the problem could be addressed in the future, should a decision be made to repeat the exercise. The following conclusions can be drawn from discussions in the previous section:

- $\mathrm{REACH}$, access to resources and $\mathrm{CO}_{2}$ /climate change and energy costs are the environmental business hazards that have been selected for testing the framework for environmental risk management. REACH and access to resources were the most prominent hazards identified from the focus group results. The energy costs hazard was selected to reflect broader concerns related to $\mathrm{CO}_{2}$ and climate change expressed within both focus groups and because a tangible impact on business objectives from the hazard could be identified, notably an increase in products costs. It will be interesting to explore how rises in energy costs may impact on engineering product cost objectives.

- Comments expressed in the focus groups suggested that identifying environmental business hazards was a valid problem that the business needs to address in future. Discussions also highlighted how it was important to understand how hazards flow through to having an impact on product designs. 
- Running the focus groups provided insights into the scope required for identifying hazards and it is concluded that there is a definite need to identify hazards with a broad scope, covering tactical and strategic problems related to the broader topic of sustainability.

- The hazards identification process also needs to include some form of horizon scanning, seeking to identify hazards as they are formulated through, for example, governmental policy making. Sources of information can be identified that may help with horizon scanning activities.

- To identify hazards a more focused approach is required, taking each life cycle stage separately, and involving participants with a greater knowledge of the topics being discussed.

- Prioritising hazards needs to be considered as a separate business activity, effectively considering the first two research problems set at the beginning of this chapter separately. Decisions should also be made by a formally recognised corporate body.

- Although it is inevitably difficult to predict the future, focus group participants felt that identifying hazards with a time horizon of up to 10 years is a realistic expectation.

The next chapters present research activities to test the framework for environmental risk management using the hazards identified. 


\section{$5 \quad$ Case study scenarios: Access to resources}

This chapter presents the research activities undertaken to test the framework for environmental risk management using the access to resources hazard identified in chapter 4. Section 5.1 presents the methodology applied for this part of the research. Section 5.2 introduces the topic of access to resources, discusses how it poses a business risk and identifies specific materials that are at high risk of supply disruptions. Section 5.3 identifies where some of these high risk materials are used in Rolls-Royce products and presents case study scenarios assessing the risk posed to the business. Section 5.4 discusses the results of the risk assessments and findings. Section 5.5 provides a summary and conclusions for this chapter, leading in to chapter 6 that presents further case study scenarios to test the framework.

\subsection{Methodology}

This section presents the research methodology applied for the research activities presented in this chapter and in chapter 6 . Research in these chapters represented a continuation of the research theme of testing the framework for environmental risk management from chapter 4 . However, a new cycle of action research was required to show how the framework could work within Rolls-Royce, assessing the environmental risk posed to product designs using case studies based on the hazards identified in chapter 4. Figure 5.1 presents the research cycle for this part of the research.

Case studies were the obvious approach for showing how the framework could work within Rolls-Royce, being a common method for undertaking qualitative empirical enquiries focused on a particular object of study within a real-world context (Dul and Hak, 2008). Similar to the different 'flavours' of action research discussed in chapter 2 , Braa and Vidgen (1999) also make a distinction between 'hard' and 'soft' case studies. Hard approaches to case studies, as described by Yin (2003), adopt a more positivistic perspective on knowledge generation. Whereas soft approaches, as discussed by Walsham (1995), take a more interpretivist approach.

Yin's positivistic stance appears to be motivated by a desire to improve the perceived validity of case study findings, enabling them to be generalised beyond the case(s) in question. Lack of generalisability is a common criticism of the case study approach (Flyvbjerg, 2011). However, those with a more interpretivist viewpoint appear to be less concerned by generalisability, instead highlighting the value of developing practical context dependent knowledge instead of theoretical context independent knowledge (Flyvbjerg, 2011). Stake (1995) makes a similar point, focusing on applying case 
studies as a means of gaining understanding and achieving change within a problematic situation, similar to action research. Given this research aims to produce outcomes that could lead to change within Rolls-Royce, this perspective on the case study approach will be adopted. This approach is also supported by findings from chapter 2, which highlighted the importance of developing an approach to integrating considerations into design decisions that was relevant to the context.

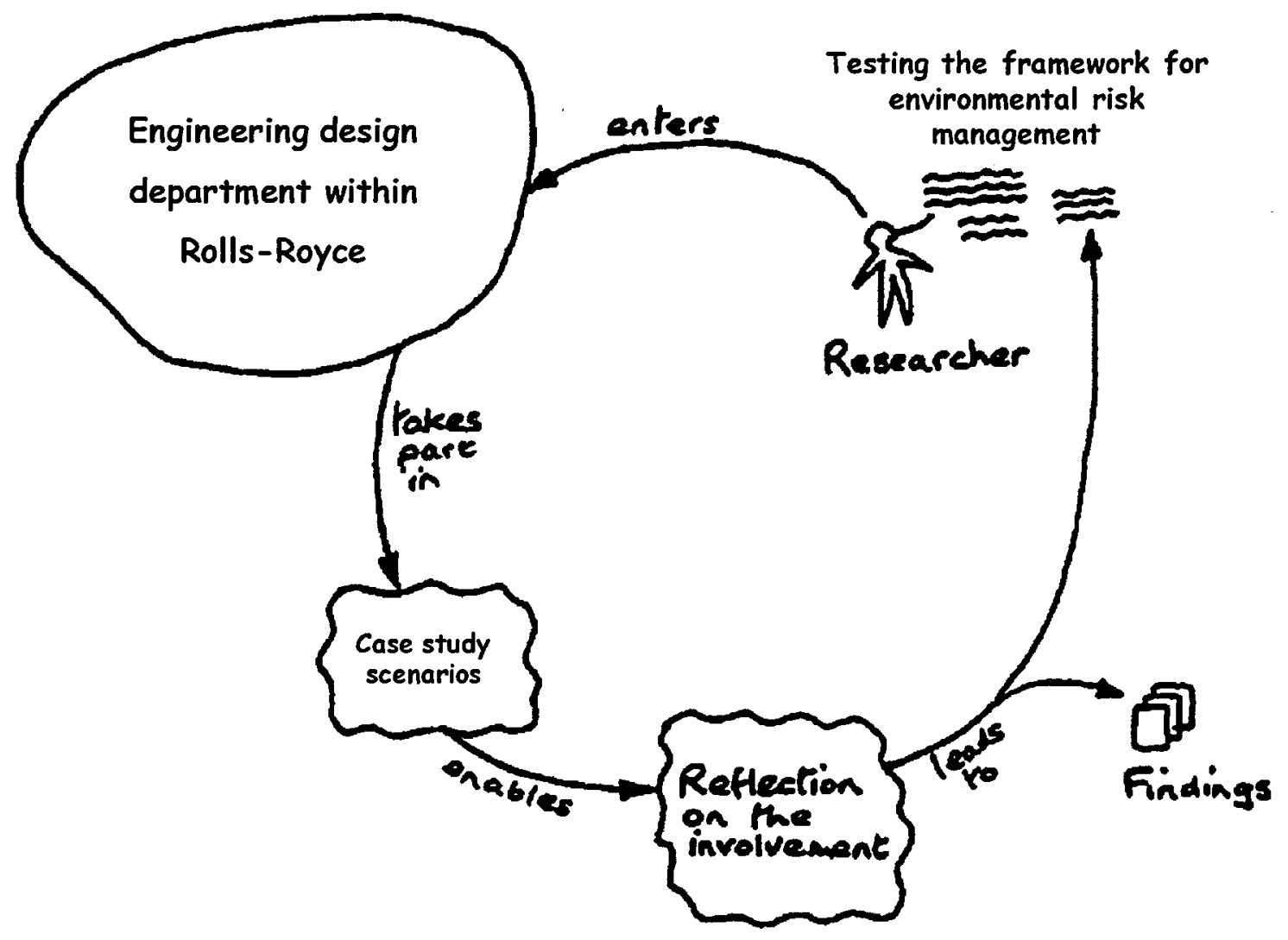

Figure 5.1: Action research cycle for testing the framework (adapted from Checkland and Holwell, 1998)

Dul and Hak (2008) provide a useful outline of the components of case study research that can be used to structure an investigation, including:

- Unit of analysis: The case. Single or multiple cases can be studied, each with or without embedded cases (a case within a case). Embedded case studies can provide detail, which can be particularly relevant when analysing organisations; the organisation itself represents a case, although it will often be necessary to analyse particular aspects of that organisation to gain any significant insights (Yin, 2003);

- Object of study: What is being studied, which could be 'activities, processes, events, persons, groups, organisations' (Dul and Hak, 2008, p.35);

- Hypotheses (if any) or research theme: A case study may not have any hypotheses if it is exploratory or descriptive. Within an action research 
approach hypotheses are replaced by research themes (Checkland and Holwell, 1998);

Applying the components of case study research for the present situation:

- Unit of analysis: Rolls-Royce was the unit of analysis within which the framework was tested. Embedded units of analysis needed to be defined to show how the framework could be used to assess the environmental risks posed to product designs, using the environmental business hazards defined in chapter 4;

- Object of study: Engineering design within Rolls-Royce, as described in chapter 2;

- Research theme: As case studies are being applied within an action research approach the validity of results are judged within a research theme, which was declared in Figure 5.1 as testing the framework for environmental risk management;

The next section provides more detail on the access to resources hazard to enable the selection of the embedded case studies within Rolls-Royce. To show how the framework works, embedded cases will need to focus on demonstrating how the risk to a Rolls-Royce product can be assessed from the access to resources hazard. As mentioned in the development of the framework in chapter 3 , this requires sufficient information on that hazard so a link with products and designs affected by it can be substantiated.

\subsection{Access to resources}

The first part of this section is largely based on information taken from Lloyd et al. (2011) and Lloyd et al. (2012c).

'Access to resources' refers to a complex phenomenon concerned with understanding the business risks posed by potential future restrictions in the accessibility of resources, in particular metals and non-fuel minerals (referred to as materials) (Lloyd et al., 2012c). Until the middle of the last decade, efforts to understand this particular problem focused on a lack of material availability due to geological scarcity (Erdmann and Graedel, 2011). Depletion indices (Lee, 1998) were used to calculate the years of a resource remaining by dividing a measure of the amount of resource left by its rate of consumption (Cohen, 2007; Tilton, 2003). Typically, indices used either static values of resource availability or applied dynamic assessments (Alonso et al., 2007). 
Whilst this appears to be simple, the problem is significantly more complex, as any estimates of the amount of a resource left contain significant uncertainties. From Gordon et al. (2007), estimates can be based either on resources, reserve base or reserves. Resources are effectively the feasibly available amount of a material in the Earth's crust. Reserve base represents resources that are deemed to be extractable with current technology. Reserves are the economically extractable parts of the reserve base at current prices. Estimates of reserves and the reserve base are governed by the potential for profitable extraction at any particular time, making the data very unreliable (Gordon et al., 2007).

To a certain extent, these uncertainties have led to a continuing debate on the availability of materials, which remains polarised between those who see depletion as a significant issue and those who have faith in technology and free markets. A view of the main arguments is available from a variety of sources; see for example Gordon et al. (2007), Morley and Eatherley (2008), Neumayer (2000), Steen (2006), Tilton (1996) and Tilton and Lagos (2007).

More recent studies suggest that the debate is moving on, adopting a new perspective and revealing a different problem. As pointed out by Rosenau-Tornow et al. (2009), and others, the question is increasingly becoming one of accessibility rather than availability. Historical examples suggest that the accessibility of resources can be affected by economic, social and environmental constraints as well as geological ones. The 'Cobalt Crisis' in the 1970s was due to conflict in Zaire (now Democratic Republic of the Congo), a key supplying region (Alonso et al., 2007). Recent interest in the topic of materials accessibility has been engendered by China dominating supplies of rare earth elements, which geologically are not that rare (National Research Council, 2008). So the question is whether or not the accessibility of resources will be affected by economic, social, environmental and geological constraints, and if so, what might be the consequences. These two factors are captured within the concept of materials 'criticality', as shown in Figure 5.2 (National Research Council, 2008). 


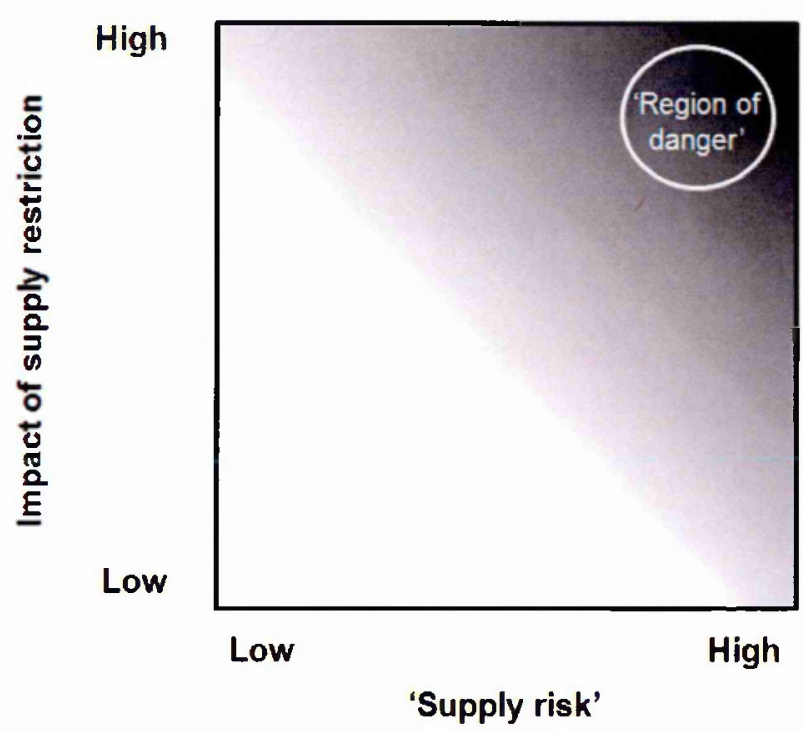

Figure 5.2: The concept of 'criticality' (National Research Council, 2008)

Materials criticality seeks to understand the risks posed by potential restrictions in the accessibility of materials by assessing the probability of a material supply restriction (often termed 'supply risk') and the potential impact if a restriction were to occur. Capturing these two measures in the 'criticality matrix' (Figure 5.2), a material is considered critical if it is high risk, i.e. the probability of a supply restriction is high and this would have significant negative consequences if it were to occur.

The two axes of the criticality diagram present the problems that have to be addressed in order to understand the risks posed by the access to resources hazard. The two axes can also be transposed onto the axes in a standard Rolls-Royce Probability Impact Diagram (PID) from chapter 3 (Figure 5.3).

\begin{tabular}{|c|c|c|c|c|c|c|}
\hline \multirow{5}{*}{ 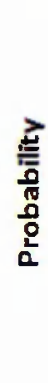 } & $\geq 50 \%$ & 9 & 14 & 19 & 24 & 29 \\
\hline & $\geq 25 \%$ to $<50 \%$ & 7 & 12 & 17 & 22 & 27 \\
\hline & $\geq 5 \%$ to $<25 \%$ & 5 & 10 & 15 & 20 & 25 \\
\hline & $\geq 1 \%$ to $<5 \%$ & 3 & 8 & 13 & 18 & 23 \\
\hline & \multirow[t]{3}{*}{$<1 \%$} & 1 & 6 & 11 & 16 & 21 \\
\hline & & Verylow & Low & Medium & High & Very High \\
\hline & & \multicolumn{5}{|c|}{ Impact } \\
\hline
\end{tabular}

Figure 5.3: Example Probability Impact Diagram (PID) (Rolls-Royce, 2007b)

Understanding the 'supply risk' of a material can be used to understand probability within the PID, which can then be multiplied by business impact to assess the risk. Hence, what is required is to identify materials that carry a high probability of supply 
restriction and identify where these are used within designs and products. This will enable an assessment of the potential impact on the business thereby completing the risk assessment.

Typical approaches to assessing the probability of a materials supply restriction apply metrics that reflect common concerns about why a material might become restricted or are derived factors that in the past have given rise to material supply restrictions (for example political instability in key supplying regions, as in the 'Cobalt Crisis'). Table 5.1 provides a summary of common metrics that have been used. Once metrics have been selected a means of scoring materials using these metrics is required. Results can then be aggregated (including the use of weighting factors if desired) to highlight materials that are more or less likely to become supply restricted in some way.

Table 5.1: Summary of supply risk metrics and underlying assumptions (Lloyd et al., 2012c)

\begin{tabular}{|l|l|}
\hline \multicolumn{1}{|c|}{ Supply risk metric } & \multicolumn{1}{c|}{ Underlying assumption } \\
\hline $\begin{array}{l}\text { Geological measures } \\
\text { (depletion indices or crustal abundance) }\end{array}$ & Low depletion index/abundance increases risk \\
\hline Co-production & Primary ore dependence increases risk \\
\hline Monopoly supply & Supply concentrations increase risk \\
\hline Political stability & Instability in producing regions increases risk \\
\hline Recyclability & Lack of recycled sources increases risk \\
\hline Substitutability & Lack of substitutability increases risk \\
\hline Environmental impact & High environmental impacts increase risk \\
\hline Demand changes & New demand increases risk \\
\hline Price volatility & High price volatility increases risk \\
\hline Social impact & Presence of social issues increases risk \\
\hline
\end{tabular}

Developing a method for assessing the probability of a materials supply restriction is a complex and time intensive task that is beyond the scope of this research, so an existing methodology is required. Discussions with Rolls-Royce elemental commodity specialists suggested the main requirement was to use a methodology that was transparent with readily available data, so the assessment could be repeated. Based on this requirement, a method developed by the European Commission (2010) was chosen (the EC report). From the studies available at the time the EC report was the only one to provide sufficient methodological transparency to allow the assessment to be repeated, backed up with an appendix containing all the background information required.

Lloyd et al. (2012a/b) provide a summary of the EC methodology applied to identify materials with a high Supply Risk (SR). Results for materials with the five highest SR 
scores, i.e. those materials with the highest probabilities of a supply restriction, are presented in Table 5.2.

Table 5.2: Top five results for Supply Risk (SR) (European Commission, 2010)

\begin{tabular}{|l|c|}
\hline \multicolumn{1}{|c|}{ Material } & SR \\
\hline Rare Earth Elements (REEs) & 4.9 \\
\hline Platinum Group Metals (PGMs) & 3.6 \\
\hline niobium & 2.8 \\
\hline germanium & 2.7 \\
\hline antimony & 2.6 \\
\hline
\end{tabular}

Identifying materials with a high supply risk allows for the access to resources hazard to be linked to a product. As discussed in the development of the framework in chapter 3, linking a hazard to a product enables the assessment of the environmental risk posed to that product. The following section presents the first two case studies, selected by identifying products that contain materials listed in Table 5.2.

\subsection{Case study scenarios}

This section presents the results of the first set of case study scenarios, assessing the risks posed to Rolls-Royce products by the access to resources hazard. The term 'case study scenario' is used as the cases presented here are pilot studies, which were not completed within real-time design situations or within the context of actual business decisions. Pilot studies were deemed to be more appropriate for testing the framework, showing how it could allow for environmental risks to be considered as part of standard design decisions in a beneficial way. Findings from these pilot studies are used to make recommendations on how to implement the framework into the business in chapter 7 . The pilot nature of the case studies is also discussed in chapter 8.

Section 5.3.1 presents the first case study identifying the use of ruthenium $(R u)$, a scarce Platinum Group Metal (PGM), in an existing product. This scenario demonstrates the framework from the perspective represented by the dashed arrows (leading from prioritised hazards to in-service products) in Figure 3.15 (p.88). Section 5.3.2 presents the second case study identifying the use of yttrium (Y), a Rare Earth Element (REE), in a new design. This demonstrates the framework from the perspective of the solid arrows (leading from design to prioritised hazard) in Figure 3.15 . 
Risk assessments presented in the following case studies have been completed by adhering as closely as possible to standard risk management practices within RollsRoyce, as discussed in chapter 3 , including:

- Structuring the risk in an 'if...then' format;

- Using risk criteria based on actual business objectives, taken from existing business risk management plans;

- Assessing risk using a standard PID based on these objectives, similar to the one shown in Figure 5.3.

Using standard risk management practices for the case studies follows on from the conclusion in chapter 3 that environmental risks should be assessed like any other risk within Rolls-Royce. The risk assessment approach is presented at the beginning of each case study, structuring the risk using an 'if...then' statement, defining the business risk management criteria and the PID to be used for the assessment.

\subsubsection{Ruthenium in the Thames engine}

Ruthenium has been designed into the Intermediate Pressure Turbine (IPT) blades on one of Rolls-Royce's newest advanced civil turbofan engines. For the purposes of commercial sensitivity, this engine will be referred to as the 'Thames', following a RollsRoyce convention for naming engines after rivers (Figure 5.4).

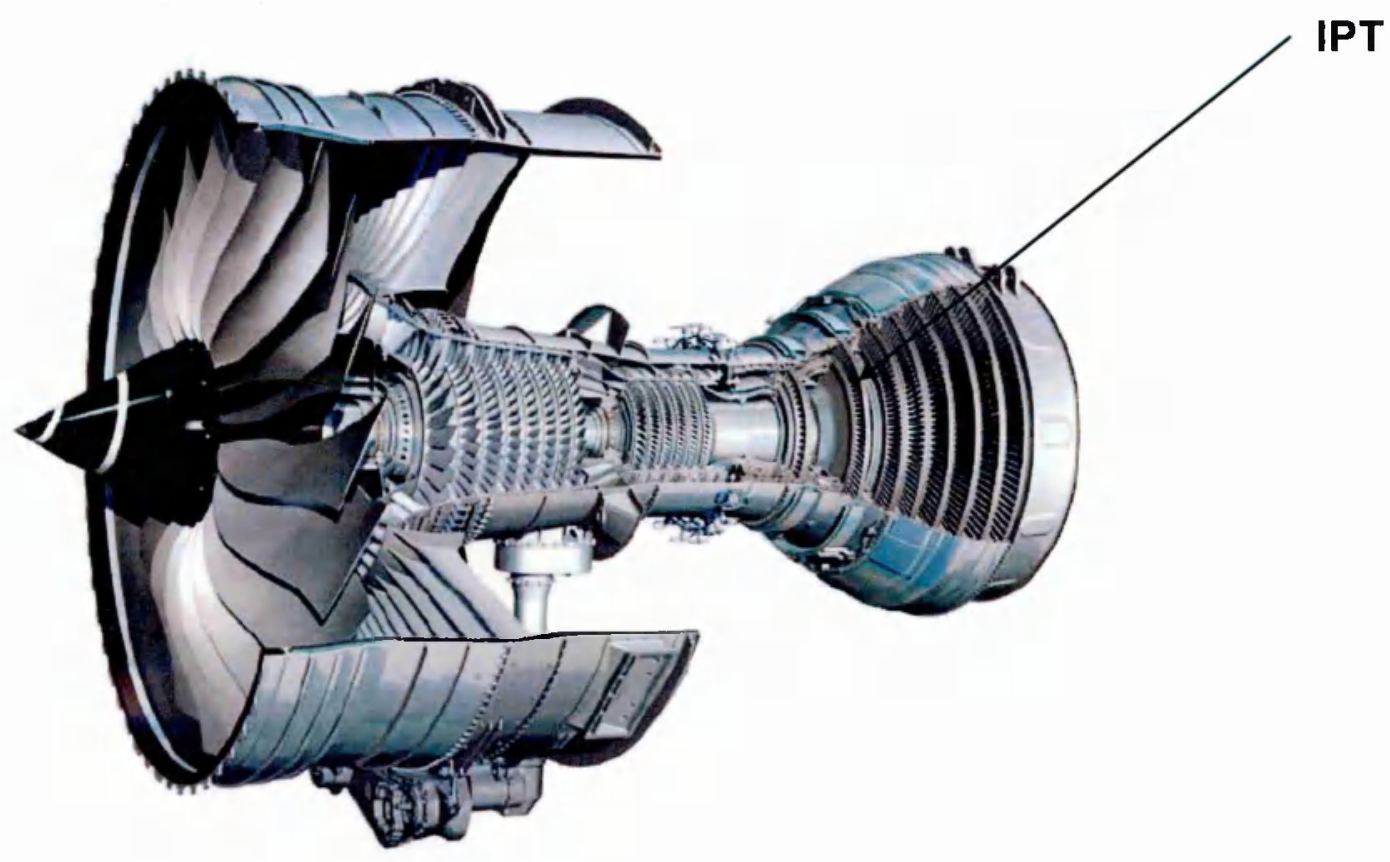

Figure 5.4: Thames engine with location of IPT highlighted (Rolls-Royce, 2011) 
Ruthenium is added to the IPT blade alloy as the alloy also contains a relatively high percentage of rhenium, which provides greater creep strength under high pressures and temperatures. However, blades with high rhenium content also have a tendency to become micro-structurally unstable, forming topologically close packed phases affecting the strength properties of the blade. Adding ruthenium to the alloy helps to prevent this from occurring, allowing a higher rhenium content and hence creep strength (Koizumi et al., 2003). During discussions it was also noted that rhenium itself was considered to have potential supply risks, which was also highlighted as a potential problem during the focus groups discussed in chapter 4 . However, the use of rhenium was overlooked for this risk assessment as it was not highlighted as a risk by the EC report. This might highlight a problem with the methodology used and this finding is discussed later in this chapter.

\subsubsection{Risk assessment approach}

Concerns over the use of ruthenium focused on potential restrictions in supply leading to significant price increases and unacceptable costs of the parts that contain ruthenium on the Thames engine. To capture these concerns, the following 'if...then' statement structures the risk assessment that needs to be completed:

\section{If...the availability of ruthenium becomes restricted in some way,}

Then... ruthenium might increase in price, impacting on Rolls-Royce's business objectives.

Criteria for the risk assessment were taken from the Thames engine risk management plan (Preston, 2010), presented in Table 5.3.

Table 5.3: Thames engine risk criteria (Preston, 2010)

\begin{tabular}{|c|c|c|c|c|c|c|c|}
\hline \multirow{2}{*}{ Category } & \multirow{2}{*}{ Probability } & \multicolumn{5}{|c|}{ Impact } & \multirow[b]{2}{*}{ Noise } \\
\hline & & Time & ENPV $^{*}$ & Unit cost & Weight & SFC $^{\star \star}$ & \\
\hline Very Low & $\leq 1 \%$ & $<1$ week & $<£ 0.5 m$ & $<£ 3 k$ & $<12$ lbs & $<12$ lbs & $<0.06 \mathrm{~dB}$ \\
\hline Low & $>1 \%$ to $\leq 5 \%$ & $\begin{array}{c}1 \text { to } 2 \\
\text { week slip } \\
\text { to plan }\end{array}$ & $\begin{aligned} \geq £ 0.5 m \text { to } & \\
& <£ 2 m\end{aligned}$ & $\begin{array}{l}\geq £ 3 k \text { to } \\
<£ 5 k\end{array}$ & $\begin{array}{c}12 \text { to } 20 \\
\text { lbs }\end{array}$ & $\begin{array}{c}12 \text { to } 20 \\
\text { Ibs }\end{array}$ & $\begin{array}{l}0.06 \text { to } \\
0.1 \mathrm{~dB}\end{array}$ \\
\hline Medium & $>5 \%$ to $\leq 25 \%$ & $\begin{array}{c}2 \text { to } 9 \\
\text { week slip } \\
\text { to plan }\end{array}$ & $\begin{array}{l}\geq £ 3.5 \mathrm{~m} \text { to } \\
<£ 17.5 \mathrm{~m}\end{array}$ & $\begin{array}{l}\geq £ 5 k \text { to } \\
<£ 25 k\end{array}$ & $\begin{array}{l}20 \text { to } \\
100 \mathrm{lbs}\end{array}$ & $\begin{array}{l}20 \text { to } 100 \\
\text { lbs }\end{array}$ & $\begin{array}{l}0.1 \text { to } \\
0.5 \mathrm{~dB}\end{array}$ \\
\hline High & $\begin{array}{l}>25 \% \text { to } \\
\leq 50 \%\end{array}$ & $\begin{array}{c}9 \text { to } 18 \\
\text { week slip } \\
\text { to plan }\end{array}$ & $\begin{array}{c}\geq £ 17.5 \mathrm{~m} \text { to } \\
\quad<£ 35 \mathrm{~m}\end{array}$ & $\begin{array}{c}\geq £ 25 k \text { to } \\
\quad<£ 50 k\end{array}$ & $\begin{array}{l}100 \text { to } \\
200 \text { lbs }\end{array}$ & $\begin{array}{l}100 \text { to } \\
200 \mathrm{lbs}\end{array}$ & $\begin{array}{c}0.5 \text { to } 1 \\
\mathrm{~dB}\end{array}$ \\
\hline Very High & $>50 \%$ & $\begin{array}{l}>18 \text { week } \\
\text { slip to } \\
\text { plan }\end{array}$ & $\geq £ 35 \mathrm{~m}$ & $\geq 50 \mathrm{~K}$ & $>200 \mathrm{lbs}$ & $>200 \mathrm{lbs}$ & $>1 \mathrm{~dB}$ \\
\hline
\end{tabular}

*Values expressed in Net Present Value (NPV) are discounted at 10\% p.a. over the Ten Year Forecast

**Specific Fuel Consumption 
Criteria cover various technical and business objectives relevant to the whole engine and programme. To create a PID for the assessment it is necessary to translate the relevant criteria onto the scales of a PID. Focusing on product, following the 'if...then' statement above, the scale for impact on unit cost ranges from very high at over $£ 50,000$ to very low at under $£ 3,000$. Scales of probability range from very high at $>50 \%$ to very low at $\leq 1 \%$. Figure 5.5 translates these scores onto a PID, which will be used for the risk assessment.

\begin{tabular}{|c|c|c|c|c|c|c|}
\hline \multirow{5}{*}{$\begin{array}{l}\text { 흠 } \\
\frac{1}{0} \\
\text { 8 } \\
0 \\
0\end{array}$} & $250 \%$ & 9 & 14 & 19 & 24 & 29 \\
\hline & $225 \%$ to $<50 \%$ & 7 & 12 & 17 & 22 & 27 \\
\hline & $25 \%$ to $<25 \%$ & 5 & 10 & 15 & 20 & 25 \\
\hline & $\geq 1 \%$ to $<5 \%$ & 3 & 8 & 13 & 18 & 23 \\
\hline & $<1 \%$ & 1 & 6 & 11 & 16 & 21 \\
\hline & & $<f 3 k$ & $\begin{array}{c}\geq f 3 k \text { to }< \\
f 5 k\end{array}$ & $\begin{array}{l}\geq f 5 k \text { to } \\
<f 25 k\end{array}$ & $\begin{array}{c}\geq £ 25 k \text { to } \\
<f 50 k\end{array}$ & $\geq £ 50 k$ \\
\hline & & \multicolumn{5}{|c|}{ Impact } \\
\hline
\end{tabular}

Figure 5.5: Thames engine unit cost PID

\subsubsection{Risk assessment}

Using the 'if...then' statement outlined, to complete the risk assessment it is necessary to assess the probability that ruthenium will be subject to a supply restriction and the impact this would have on the business if this were to occur.

As discussed in section 5.2, understanding the 'supply risk' of a material can be used to assess the probability of a material supply restriction. Results produced by the EC report were provided in Table 5.2. What is required is to translate these scores so they can be used within the risk assessment PID shown in Figure 5.5. Table 5.4 shows the scoring scheme developed based on the actual results of the EC report (rather than the theoretical maximum and minimum scores). Platinum group metals scored a SR of 3.6 from the EC report, translating in to a high probability (25\% to $50 \%$ ) risk. 
Table 5.4: Probability assessment

\begin{tabular}{|c|c|}
\hline Probability (P) & SR score \\
\hline$\geq 50 \%$ & $\mathrm{SR} \geq 4$ \\
\hline$\geq 25 \%$ to $<50 \%$ & $3 \leq \mathrm{SR}<4$ \\
\hline$\geq 5 \%$ to $<25 \%$ & $2 \leq \mathrm{SR} \leq 3$ \\
\hline$\geq 1 \%$ to $<5 \%$ & $1 \leq \mathrm{SR}<2$ \\
\hline$<1 \%$ & $\mathrm{SR}<1$ \\
\hline
\end{tabular}

To assess the impact of a supply restriction in terms of product cost requires answers to the following three questions:

1. What is the cost of ruthenium used within an IPT blade on the Thames engine?

2. How might the price of the material change?

3. How does this affect product cost and impact on business objectives?

Addressing the first question, calculating the cost of the ruthenium in an IPT blade requires the cost of ruthenium and the amount of ruthenium used in manufacturing the blades. An illustrative cost of ruthenium was used, as company cost information is commercially sensitive. The amount of ruthenium used to manufacture the blade was estimated using the weight of the blade (taken from internal engineering data), an estimate of material losses in manufacture (blades are investment cast, a figure was taken from a manufacturing specialist) and the alloy composition. Presented in Table 5.5 , the cost of ruthenium per blade was estimated at just under $£ 45$.

Table 5.5: Calculating the cost of ruthenium $(R u)^{2}$

\begin{tabular}{|l|c|l|}
\hline Weight (lbs) & 0.6 & Finished blade \\
\hline Input weight (lbs) & 0.7 & Total material required \\
\hline Weight of Ru (lbs) & 0.024 & 4 wt\%, from alloy composition \\
\hline Material cost (£/lb) & 1600 & Illustrative cost \\
\hline Cost of Ru & $£ 44.80$ & \\
\hline
\end{tabular}

The next task is to assess how the price of ruthenium might change and how this translates into an impact on unit cost. Attempting to predict the future price of a raw material presents a very difficult challenge. This type of problem would typically be approached by using statistical data of past prices and extrapolating this into the future, although this would require modelling effort that is beyond the scope of this thesis. There is also well founded scepticism over the value of relying on past trends to predict future prices (Taleb et al., 2009).

\footnotetext{
${ }^{2}$ Due to commercial sensitivity, data presented is indicative rather than absolute.
} 
A simpler measure of price volatility may be used, defined as a ratio of the maximum to minimum market price for a material (i) over a fixed time period (Rolls-Royce use 10 years), as shown in Equation 5.1:

$$
P V_{i}=\text { Max.price } / \text { Min.price }_{10 \text { years }} \quad \text { (Equation 5.1) }
$$

Applying price volatility is justified on the basis that the measure has been used as a component in assessing supply risk within criticality assessments (as listed in Table 5.1). From discussions with an elemental purchasing specialist, Rolls-Royce also analyses price volatility to inform short term metal price risk mitigation strategies.

An illustrative 10 year price volatility of ruthenium of 20 is used, as the original figures used were deemed to be commercially sensitive. Multiplying this figure by the cost of ruthenium calculated in Table 5.5 gives a cost impact of just under $£ 900$ per blade. There are $120^{3}$ IPT blades used in the Thames engine. Multiplying the impact per blade by the number of blades in the engine gives a total potential engine unit cost impact of $£ 107,520$ (Table 5.6).

Table 5.6: Ruthenium impact calculations ${ }^{3}$

\begin{tabular}{|l|c|l|}
\hline Ru max. Price (\$/oz) & 800 & \\
\hline Ru min. Price (\$/oz) & 40 & \\
\hline Price volatility & 20 & \\
\hline Cost of Ru & $£ 44.80$ & Table 5.5 \\
\hline New cost of Ru & $\mathfrak{£ 8 9 6}$ & \\
\hline Engine unit cost impact & $\mathfrak{£ 1 0 7 , 5 2 0}$ & 120 blades per engine \\
\hline
\end{tabular}

The risk assessment can be completed by plotting the scores of probability and impact on the PID given in Figure 5.5. With a high probability (25\% to $50 \%)$ and an impact of $£ 107,520$ the risk assessment score for the use of ruthenium is 27 , circled in Figure 5.6. A risk of this magnitude would be classed as significant, requiring mitigating actions.

\footnotetext{
${ }^{3}$ Modified from actual values to protect sensitive commercial data.
} 


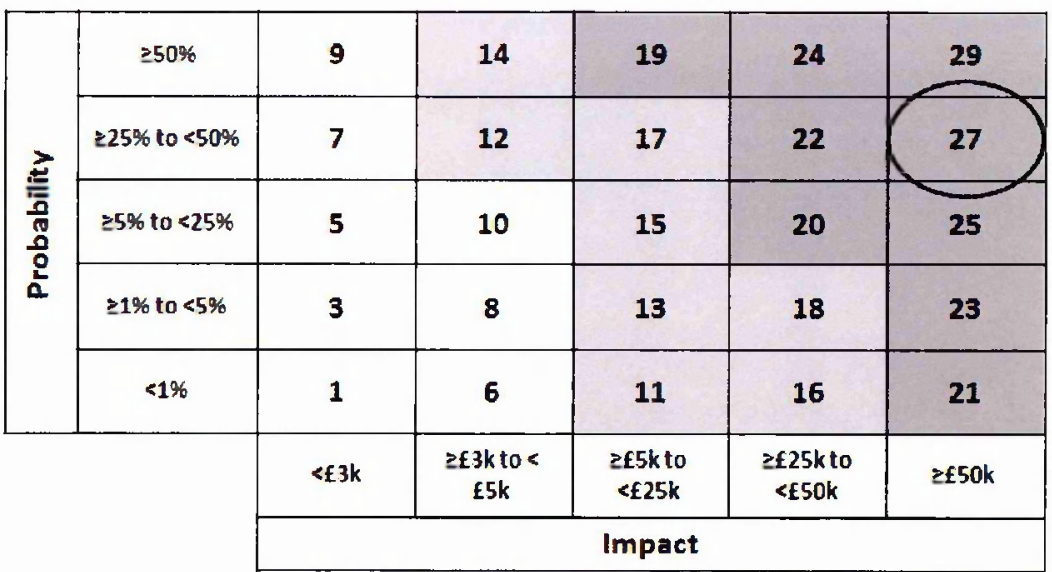

Figure 5.6: Use of ruthenium risk assessment result

\subsubsection{Sensitivity analysis}

It is important to explore sensitivities to give an indication of how robust the risk assessment result is, which are explored here. Several significant assumptions had to be made to produce a risk assessment result. These assumptions create uncertainties in the assessment of probability and impact.

Starting with the assessment of probability first, the European Commission method for assessing the probability of a supply restriction was selected on the basis of it being an established methodology with readily available data. It is interesting to analyse how using a different approach affects the risk assessment result. A more recent study by the British Geological Survey (BGS, 2011) offers an alternative assessment. This method was overlooked for the initial assessment as no background data is available from the BGS to re-produce results. Adopting a slightly simpler methodology than the EC report, the following metrics are used to assess the probability of a materials supply restriction:

- Geological: applying the crustal abundance of a material measured in parts per million (ppm);

- Monopoly supply: measured using the percentage of production from the top three producing countries;

- Monopoly supply: applying a further measurement based on the percentage of the global reserve base held by the top three countries;

- Political stability: applying the same WGI indicators as used in the EC report (Kaufmann et al., 2010).

Materials were scored against each metric on a scale from one to five, five being high risk, as shown in Table 5.7. 
Table 5.7: BGS methodology (BGS, 2011)

\begin{tabular}{|c|c|c|c|c|}
\hline $\begin{array}{c}\text { Risk } \\
\text { score }\end{array}$ & $\begin{array}{c}\text { Scarcity } \\
\text { (ppm) }\end{array}$ & $\begin{array}{c}\text { Production concentration } \\
(\%)\end{array}$ & $\begin{array}{c}\text { Reserve base distribution } \\
\text { (\%) }\end{array}$ & $\begin{array}{c}\text { Governance } \\
\text { (index) }\end{array}$ \\
\hline 1 & $>100$ to 1000 & 0 to 30 & 0 to 30 & 0 to 2 \\
\hline 2 & $>10$ to 100 & $>30$ to 45 & $>30$ to 45 & 3 to 4 \\
\hline 3 & $>1$ to 10 & $>45$ to 60 & $>45$ to 60 & 5 to 6 \\
\hline 4 & $>0.1$ to 1 & $>60$ to 75 & $>60$ to 75 & 7 to 8 \\
\hline 5 & $<0.1$ & $>75$ & $>75$ & $8+$ \\
\hline
\end{tabular}

Materials were scored against each metric and results aggregated and divided by two, producing a simple risk index on a scale of 0 to 10 . Sample scores produced are presented in Table 5.8 .

Table 5.8: Sample scores from the BGS method (BGS, 2011)

\begin{tabular}{|l|c|}
\hline Material & Supply risk index \\
\hline Platinum Group Metals (PGMs) & 8.5 \\
\hline beryllium & 6.5 \\
\hline cobalt & 5.5 \\
\hline zirconium & 4.5 \\
\hline titanium & 2.5 \\
\hline
\end{tabular}

As before, it is necessary to translate the BGS supply risk scores into a scale consistent with the PID in Figure 5.5. Unlike the European Commission method the results produced do not fit neatly into a linear scale. The scoring scheme shown in Table 5.9 was developed based on the boundaries drawn within the risk list produced by the BGS (see BGS, 2011).

Table 5.9: Risk scoring scheme for BGS method, with PGM score highlighted

\begin{tabular}{|c|c|}
\hline Probability (P) & SR score \\
\hline$\geq 50 \%$ & SR $>7.5$ \\
\hline$\geq 25 \%$ to $<50 \%$ & $6<\mathrm{SR} \leq 7.5$ \\
\hline$\geq 5 \%$ to $<25 \%$ & $5<\mathrm{SR} \leq 6$ \\
\hline$\geq 1 \%$ to $<5 \%$ & $4 \leq \mathrm{SR} \leq 5$ \\
\hline$<1 \%$ & $\mathrm{SR}<4$ \\
\hline
\end{tabular}

PGMs scored 8.5 in the BGS assessment, translating into a very high $(>50 \%)$ probability. Plotted with the assessment of impact this gives a risk score of 29 (Figure 5.7), which is the highest possible risk score. Again the risk would still be classified as a key threat, requiring mitigating actions. 


\begin{tabular}{|c|c|c|c|c|c|c|}
\hline \multirow{5}{*}{ 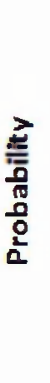 } & $250 \%$ & 9 & 14 & 19 & 24 & 29 \\
\hline & $225 \%$ to $<50 \%$ & 7 & 12 & 17 & 22 & 27 \\
\hline & $\geq 5 \%$ to $<25 \%$ & 5 & 10 & 15 & 20 & 25 \\
\hline & $\geq 1 \%$ to $<5 \%$ & 3 & 8 & 13 & 18 & 23 \\
\hline & $<1 \%$ & 1 & 6 & 11 & 16 & 21 \\
\hline & & $<E-3 k$ & $\begin{array}{c}\geq f 3 k \text { to }< \\
f 5 k\end{array}$ & $\begin{array}{l}\geq f 5 k \text { to } \\
<f 25 k\end{array}$ & $\begin{array}{c}\geq f 25 \mathrm{k} \text { to } \\
<\mathrm{f} 50 \mathrm{k}\end{array}$ & $\geq f 50 \mathrm{k}$ \\
\hline
\end{tabular}

Figure 5.7: Risk assessment result using BGS probability assessment method

Sensitivities around the measurement of impact relate to the costs of the raw materials used and the method for estimating how the price of materials might change. Calculations of raw material costs for ruthenium are fair, given both the cost and weight data was taken from reliable internal sources. Greater uncertainties lie in assessing how the price of a material might change. As discussed, attempting to estimate the future price of a material to any degree of certainty is very challenging, if not impossible. It is recognised that applying the simple measure of price volatility to give an indication of how raw material prices might change is imperfect, although it is claimed as fair, providing a simplified means of extrapolating past price trends into the future to give an indication of the potential change in price. Ruthenium has a very high price volatility and it is worth questioning whether or not the price paid by Rolls-Royce would ever actually change by this much. However, a price change of half the amount calculated would produce a cost impact of greater than $£ 50,000$, which would still be judged as significant using the PID defined in Figure 5.5.

An analysis of the sensitivities in this case suggests that the risk assessment result is fair. Using ruthenium presents a serious risk that the business needs to address.

\subsubsection{Treatment actions and residual risks}

Actions are required to treat the risk posed by the use of ruthenium in the Thames engine. The most obvious step is to remove it from the engine and use a different turbine blade alloy. However, this mitigating action carries residual risks, which are explored here.

Using a different turbine blade alloy would have a performance effect (measured in terms of specific fuel consumption) on the engine, reducing thermal efficiency by lowering the turbine inlet temperature at which the blades could operate. The following 
'if...then' statement is used to conduct a residual risk assessment associated with removing ruthenium from IPT blades by using a different alloy:

If... a turbine blade alloy is used that does not contain ruthenium,

Then...there will be a reduction in temperature capability of the alloy, reducing thermal efficiency and increasing specific fuel consumption (sfc).

Criteria for assessing the risks to sfc were presented in Table 5.3 and are used to create the PID shown in Figure 5.8. As before, an assessment of the probability and impact is required to understand the risk.

\begin{tabular}{|c|c|c|c|c|c|c|}
\hline \multirow{5}{*}{ 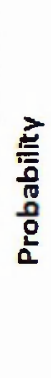 } & $250 \%$ & 9 & 14 & 19 & 24 & 29 \\
\hline & $225 \%$ to $<50 \%$ & 7 & 12 & 17 & 22 & 27 \\
\hline & $\geq 5 \%$ to $<25 \%$ & 5 & 10 & 15 & 20 & 25 \\
\hline & $\geq 1 \%$ to $<5 \%$ & 3 & 8 & 13 & 18 & 23 \\
\hline & \multirow[t]{3}{*}{$<1 \%$} & 1 & 6 & 11 & 16 & 21 \\
\hline & & $<0.03$ & 0.03 to 0.05 & 0.05 to 0.25 & 0.25 to 0.5 & $>0.5$ \\
\hline & & \multicolumn{5}{|c|}{ Impact: specific fuel consumption (\%) } \\
\hline
\end{tabular}

Figure 5.8: Risk assessment PID using sfC criteria

A reduction in sfc from removing ruthenium from the turbine blades is a certainty. However, given the reduction in sfc will only occur if ruthenium is removed, which is dependent on the probability assessment for ruthenium, the probability score from the previous risk assessment is used. Based on the results of the EC report, this produced a probability score of high $(25 \%$ to $50 \%)$.

Engineers within Rolls-Royce calculated that the reduction in sfc from using a turbine blade alloy without ruthenium would be $0.1-0.2 \%$. This translates to a medium impact using the PID shown in Figure 5.8, producing a risk score of 17 when combined with the assessment of probability (Figure 5.9). 


\begin{tabular}{|c|c|c|c|c|c|c|}
\hline \multirow{5}{*}{ 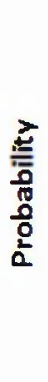 } & $250 \%$ & 9 & 14 & 19 & 24 & 29 \\
\hline & $225 \%$ to $<50 \%$ & 7 & 12 & 17 & 22 & 27 \\
\hline & $25 \%$ to $<25 \%$ & 5 & 10 & 15 & 20 & 25 \\
\hline & $21 \%$ to $<5 \%$ & 3 & 8 & 13 & 18 & 23 \\
\hline & \multirow[t]{3}{*}{$<1 \%$} & 1 & 6 & 11 & 16 & 21 \\
\hline & & $<0.03$ & 0.03 to 0.05 & 0.05 to 0.25 & 0.25 to 0.5 & $>0.5$ \\
\hline & & \multicolumn{5}{|c|}{ Impact: specific fuel consumption (\%) } \\
\hline
\end{tabular}

Figure 5.9: sfc risk assessment result

This result shows that the risks to cost of incorporating ruthenium into the Thames engine outweigh the sfc benefits provided by including the material. Considering these risks ruthenium should be taken out of the engine.

\subsubsection{Summary}

This section has presented the first case study scenario, showing how the risks posed to an in-service product from the access to resources hazard could be assessed. The risk assessment showed that the risk posed to business objectives by including ruthenium within the IPT blades of the Thames engine is significant, even when balanced against the sfc benefits of including the material. The next section presents another case study assessing the risks posed to another design by the same hazard. Results are discussed in section 5.4 .

\subsubsection{Yttrium in the Severn engine}

The second case study scenario focuses on a re-design of the outer liner of the combustor on a small military turbofan engine, which for reasons of commercial sensitivity shall be referred to as the 'Severn' engine (following the naming convention from the previous case study). Figure 5.10 provides a 3D model of a typical combustor module for a military turbofan. 


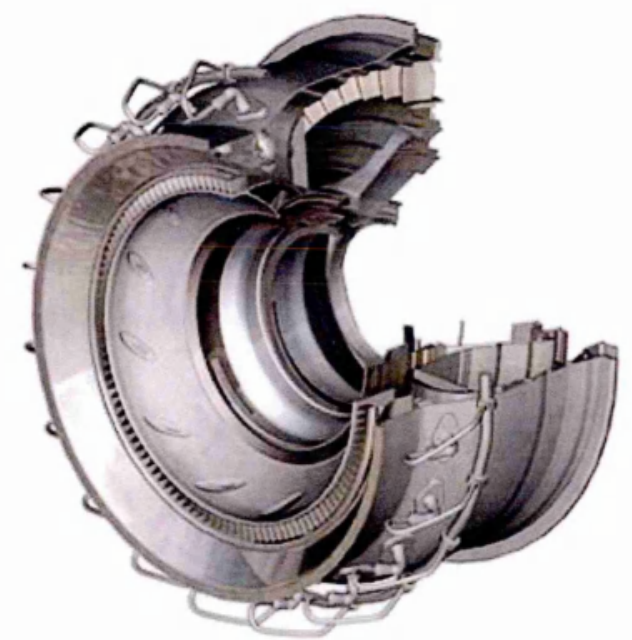

Figure 5.10: Low-bypass ratio turbofan combustion module (Rolls-Royce, 2007a)

Figure 5.11 shows a cross-section of the combustor, identifying the outer liner that was being re-designed. The combustion chamber is assembled from three main components: the inner liner, outer liner and combustor head. The two liners are rings of sheet metal that are typically forged and welded together, then being welded to the combustor head. The liners contain holes through which air from the high pressure compressor passes, being ignited within the chamber and forced out through the high pressure turbine. The outer liner is manufactured from a nickel superalloy, is approximately $500 \mathrm{~mm}$ in diameter and weighs around $7 \mathrm{~kg}$.

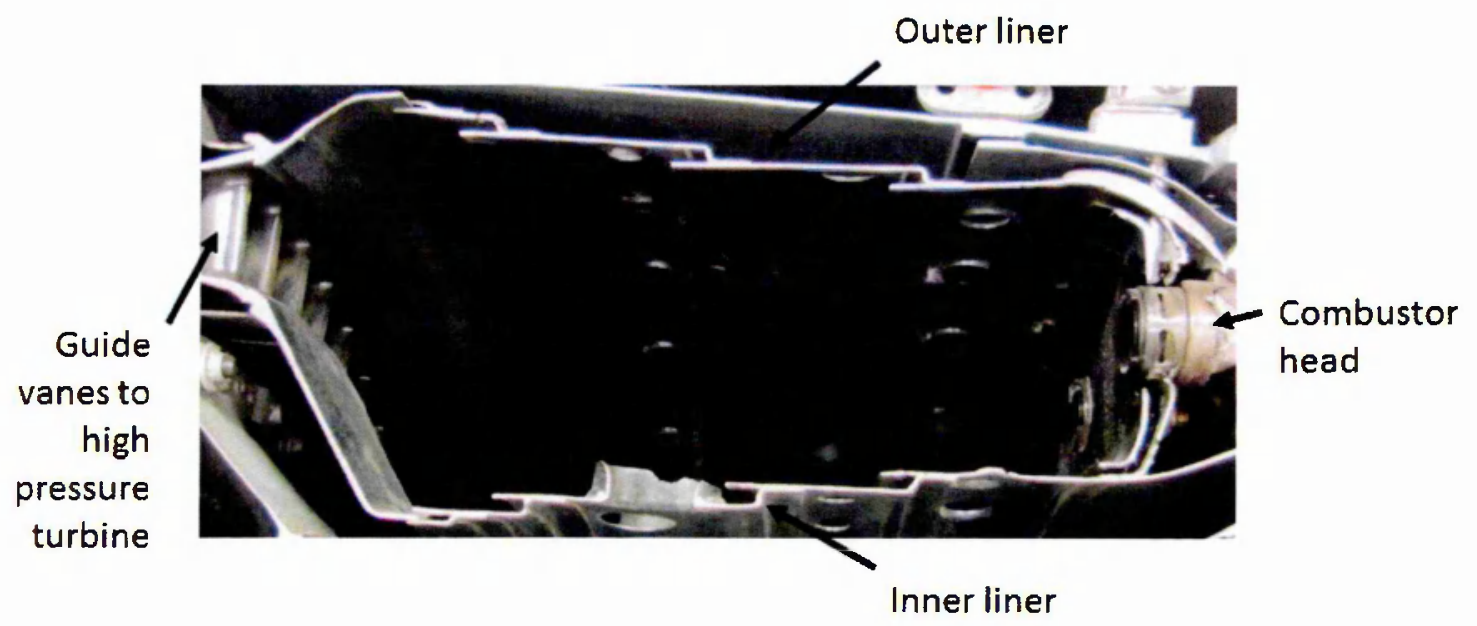

Figure 5.11: 'Severn' engine combustion chamber cross-section

During the re-design exercise it was identified that a thermal barrier coating applied to the new liner contained yttrium, a Rare Earth Element (REE). 


\subsubsection{Risk assessment approach}

As with the previous case study, concerns over the use of yttrium are focused on potential restrictions in supply leading to significant price increases and unacceptable part costs. To capture these concerns, the following 'if...then' statement structures the risk assessment that needs to be completed:

\section{If...the accessibility of yttrium becomes restricted in some way,}

Then... yttrium might increase in price, impacting on Rolls-Royce's business objectives'.

Detailed risk management plans for the Severn engine were not available for the risk assessment so risk criteria are extrapolated from the Thames engine risk management plan (as shown in Table 5.3). From Table 5.3 the scale of impact for unit cost ranges from very high at over $£ 50,000$ to very low at under $£ 3,000$. Engineering specialists within Rolls-Royce estimated that the engine set value of a large civil turbofan engine is approximately five to six times more than a small defence turbofan. To extrapolate the unit cost criteria from the Thames to the Severn engine values are scaled down by a factor of 5, producing the unit cost PID shown in Figure 5.12.

\begin{tabular}{|c|c|c|c|c|c|c|}
\hline \multirow{5}{*}{ 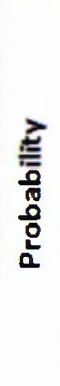 } & $\geq 50 \%$ & 9 & 14 & 19 & 24 & 29 \\
\hline & $\geq 25 \%$ to $<50 \%$ & 7 & 12 & 17 & 22 & 27 \\
\hline & $25 \%$ to $<25 \%$ & 5 & 10 & 15 & 20 & 25 \\
\hline & $\geqslant 1 \%$ to $<5 \%$ & 3 & 8 & 13 & 18 & 23 \\
\hline & \multirow[t]{3}{*}{$<1 \%$} & 1 & 6 & 11 & 16 & 21 \\
\hline & & $<f 0.6 k$ & $\begin{array}{c}2 \mathrm{f0} .6 \mathrm{k} \text { to }< \\
\text { f1k }\end{array}$ & $\begin{array}{c}\geq f 1 k \text { to } \\
<f 5 k\end{array}$ & $\begin{array}{l}\text { Zf5k to } \\
<\notin 10 k\end{array}$ & $2 f 10 \mathrm{k}$ \\
\hline & & \multicolumn{5}{|c|}{ Impact } \\
\hline
\end{tabular}

Figure 5.12: PID for Severn engine risk assessment

\subsubsection{Risk assessment}

Using the 'if...then' statement defined, to complete the risk assessment, it is necessary to assess the probability that yttrium will be subject to a supply restriction and the impact this would have on the business if it were to occur.

Beginning with probability, a method for assessing the probability of a material supply restriction was presented in Table 5.4 and the same approach can be applied for 
assessing the risks posed by the use of yttrium. REEs were deemed to be the highest risk elements from the EC report with a SR score of 4.9. From the scale in Table 5.4 this scores a very high (>50\%) probability.

Calculating impact can also be based on the same approach as applied in the previous case study, answering the following three questions:

1. What is the cost of yttrium used in the combustor outer liner on the Severn engine?

2. How might the price of the yttrium change?

3. How does this affect product cost and impact on business objectives?

Addressing the first question, calculating the cost of the yttrium used on the outer liner requires the cost of yttrium and the amount used during manufacture. The cost of yttrium was found via an internet search (Kidela, 2011), taking an average from the last three years. The amount of yttrium was estimated using the thickness of coating applied and the coating composition. From alloy composition data, $10 \%$ of the coating was yttrium. The weight of coating used was calculated using the coating thickness, simple geometry and costing density. Presented in Table 5.10, the cost of yttrium applied to the Severn engine outer liner was estimated at under $£ 2$.

Table 5.10: Calculating the cost of yttrium $(Y)$

\begin{tabular}{|l|c|l|}
\hline Weight $(\mathrm{kg})$ & 0.42 & $\begin{array}{l}\text { Indicative value calculated using simple } \\
\text { geometry }\end{array}$ \\
\hline Weight of $\mathrm{Y}(\mathrm{kg})$ & 0.042 & $10 \mathrm{wt} \%$, from alloy composition \\
\hline Material cost $(£ / \mathrm{kg})$ & 40 & Kidela (2011), 3 year estimated average \\
\hline Cost of $\mathrm{Y}$ & $£ 1.68$ & \\
\hline
\end{tabular}

The next task is to offer an impression of how the price of yttrium might change and to assess how this translates into an impact on cost. As discussed previously this presents a complex problem and the approach taken is to use historical price volatility to offer an impression of how the price of a material might change over time. As before, the volatility is calculated by dividing the maximum market price by the minimum market price over a ten year period.

Using equation 5.4 and data from Kidela (2011) the ten year price volatility for yttrium is 7. Multiplying this figure by the cost of yttrium calculated in Table 5.10 gives a cost impact of just over $£ 10$ per liner, which is the potential impact on total unit cost as there is only 1 liner per engine (Table 5.11). 
Table 5.11: Yttrium impact calculations

\begin{tabular}{|l|c|l|}
\hline$Y$ max. Price (\$) & 158 & Kidela (2011) \\
\hline Y min. Price (\$) & 25 & Kidela (2011) \\
\hline Price volatility & 7 & \\
\hline Cost of $Y$ & $£ 1.68$ & Table 5.10 \\
\hline New cost of $Y$ & $£ 11.76$ & \\
\hline Engine unit cost impact & $£ 10.08$ & 1 liner per engine \\
\hline
\end{tabular}

The risk assessment can be completed by plotting the scores of probability and impact on the chart given in Figure 5.12. With a very high (>50\%) probability and an impact of around $£ 10$ (very low), the risk assessment score for the use of yttrium is 9 , circled in Figure 5.13. Even though yttrium has a high probability of a supply restriction, given it has such a low impact on business objectives, it is likely to be disregarded as a risk.

\begin{tabular}{|c|c|c|c|c|c|c|}
\hline \multirow{5}{*}{ 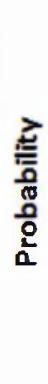 } & $250 \%$ & 9 & 14 & 19 & 24 & 29 \\
\hline & $\geq 25 \%$ to $<50 \%$ & 7 & 12 & 17 & 22 & 27 \\
\hline & $\geq 5 \%$ to $<25 \%$ & 5 & 10 & 15 & 20 & 25 \\
\hline & $\geq 1 \%$ to $<5 \%$ & 3 & 8 & 13 & 18 & 23 \\
\hline & $<1 \%$ & 1 & 6 & 11 & 16 & 21 \\
\hline & & $<f 0.6 \mathrm{k}$ & $\begin{array}{l}\geq f 0.6 k \text { to } \\
\text { f1k }\end{array}$ & $\begin{array}{c}\geq E 1 k \text { to } \\
<E 5 k\end{array}$ & $\begin{array}{l}\text { 2E5k to } \\
<E 10 \mathrm{k}\end{array}$ & Zf10k \\
\hline
\end{tabular}

Figure 5.13: Risk assessment result for the use of yttrium on the Severn engine

\subsubsection{Sensitivity analysis and treatment actions}

As in the previous case study, several significant assumptions had to be made to calculate probability and impact, which creates uncertainties in the risk assessment result. Given the risk assessment result had such a low impact on business objectives an assessment of sensitivity is not likely to reveal any significant findings in this case. Working backwards from Table 5.11, for the price of yttrium to have a significant impact on unit cost objectives in the combustor liner application (taking the $£ 600$ threshold from the PID used) it would have to rise to almost $£ 14,300$ per $\mathrm{kg}$, which is very unlikely at any point in the foreseeable future. No specific treatment actions need to be defined, requiring the assessment of residual risks. 


\subsubsection{Summary}

This section has presented the second case study scenario, showing how risks posed to a new design from the access to resources hazard can be assessed. The risk assessment showed that the risk posed to business objectives by including yttrium within the combustor of the Severn engine is insignificant.

\subsection{Discussion}

This section discusses the results of the case studies and findings. Discussions of the case studies results and findings are separated into the following topics:

- Results of the risk assessments;

- Risk assessment approach, including the need for life cycle data within the framework;

- Challenges presented in the assessments of probability and impact.

Section 5.4.5 summarises discussions with recommendations for managing risks posed by the access to resources hazard.

\subsubsection{Results}

The result from the first case study shows that the access to resources hazard can pose a significant risk to the business and needs to be considered within design decision making. Risks related to the use of ruthenium in IPT blades produced a significant result, requiring mitigating actions. However, the second case study shows that risks will not be significant in all circumstances, in particular where material costs form a small fraction of the overall unit cost of parts. Risks posed to the Severn engine due to the use of yttrium in the outer liner coating produced a very low impact score. This is interesting, as the EC report scored yttrium as having a greater probability of a supply restriction than ruthenium and it might have been expected that the risk posed to the business from using yttrium would be higher. What this highlights is that RollsRoyce risk assessment PIDs are weighted towards those risks that pose a higher impact than those that have a higher probability. Hence, to manage the risks posed by the access to resources hazard it would be sensible to focus efforts on where materials with a high probability of a supply restriction and are used in applications where the costs of these materials form a larger fraction of the overall unit cost of parts. Such an example would be when the material is used as part of a blade alloy, instead of in a coating. 
A significant outcome came from completing the residual risk assessment as part of treatment actions within the first case study. The residual risk assessment focused on assessing risk posed to sfc targets from taking ruthenium out of the IPT blades. Producing a lower risk than that posed to unit cost from the use of ruthenium, on balance ruthenium should be taken out of the engine. However, what this also shows is that the risk approach allows for environmental considerations to be trade-off decisions with other design requirements within design decision-making. This was an important requirement for the development of the DfE capability described at the beginning of this thesis.

Discussions with senior managers at Rolls-Royce confirmed that the decision to take ruthenium out of the Thames engine had been made, due to accessibility concerns that have led to significant price increases. However, this decision has only been made after over 120 engines had been produced containing the material, creating significant costs for the business. If the risk assessment had been performed when the engine was being designed (i.e. if the framework for environmental risk management had been in place at the time), a different, or at least a better informed, decision could have been made, potentially saving the business significant costs.

\subsubsection{Risk assessment approach}

The first problem that needed to be addressed to complete the risk assessments was defining the embedded case studies by identifying products that contained, or were being designed to contain, the materials listed at the end of section 5.2. 'Linking' hazards with products, by identifying some feature of that hazard (for example a material at risk of a supply disruption), was a key feature of the description of the development of the framework in chapter 3. In the case studies, ruthenium in the Thames engine was discovered through discussions with elemental commodity specialists. Yttrium in the Severn engine was discovered by pure chance whilst reviewing old design data. If the framework were put into practice, the need to link hazards with products would be fulfilled by using life cycle environmental data, similar to that produced by the initial DfE tool described in chapter 1. Features of hazards would be flagged within the materials and processes selected for a design, prompting the need for a risk assessment. Further discussion on the need for life cycle environmental data within the framework is provided in chapter 7 .

To keep the case studies as realistic as possible, and following on from the conclusion in chapter 3 to assess environmental risks like any other risk, risk assessments were 
completed by adhering as closely as possible to standard risk management practices. Risks were structured using an 'if...then' format and actual business risk criteria were used to create a typical risk assessment PID. Actual risk criteria were not available for the Severn engine, as it was based on a historical design task. Extrapolating from the Thames engine criteria (which is available as the engine is still under development) created a set of risk criteria that were defensible. Discussions with Chief Design Engineers highlighted that defining risk scoring criteria down to a design level is a challenge that the business is still getting to grips with and often generic criteria taken from other risk management plans are used. To overcome these difficulties it is often highlighted that the criteria should be used relatively to highlight what is important, rather than getting hung-up on the specific detail of the numbers. Given this, extrapolating criteria is not a significant diversion from standard risk management practice.

\subsubsection{Assessing probability}

Assessments of probability were based on an approach developed by the European Commission (2010), in particular the method for calculating 'supply risk' (which is synonymous for the probability of a supply restriction). As discussed by Lloyd et al. (2012c), there are several problems with relying on external assessments of material supply risk in this way. Many metrics can be used to assess supply risk (as listed in Table 5.1), with various ways of scoring and aggregating results to highlight materials that may be at a greater risk of a supply restriction. There are more than a dozen critical material studies (including the approach developed by the BGS used for the sensitivity analysis), which all take a slightly different view on the problem, highlighting different materials as being more or less critical.

An interesting point from the Thames engine cases study was the fact that ruthenium was included in the alloy to allow for the inclusion of a greater content of rhenium. Discussions with materials commodity specialists suggested that rhenium itself should be considered a critical material, although it was overlooked for the assessment as it was not highlighted as high risk by the EC report. This highlights the need to develop context dependent methods for assessing the probability of a material supply restriction, identifying critical materials relevant to Rolls-Royce. Having looked at these issues qualitatively for some time, elemental commodity specialists reveal valuable insights into what materials are of concern, and why. These insights could be used to build an assessment method that reflects the actual concerns of an organisation, rather than relying on external assessments and applying these results arbitrarily. Through 
their analysis, Lloyd et al. (2012c) created a framework for completing criticality assessments, presented in Figure 5.14, which could be applied to build an assessment method.

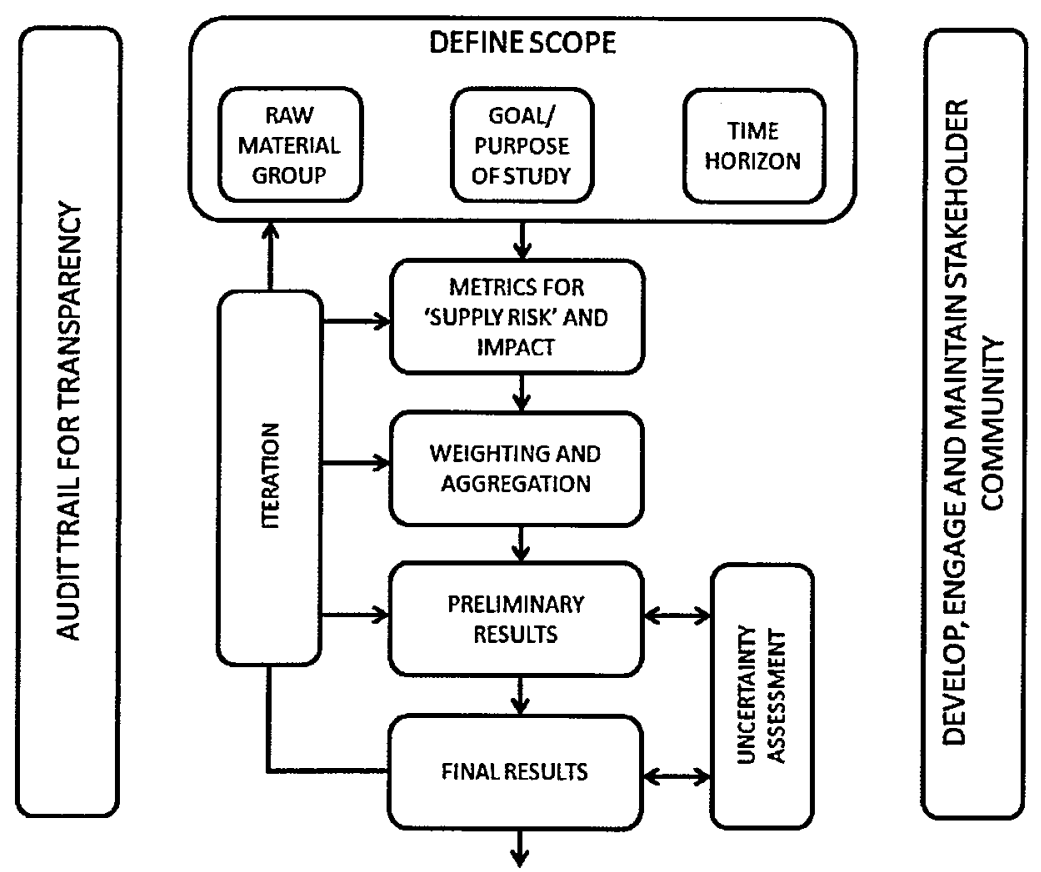

Figure 5.14: Framework for criticality assessments (Lloyd et al., 2012c)

It will still be necessary to collect data relevant to the metrics selected and develop the scoring methodology to produce an assessment, which is a time consuming and complex task. Rolls-Royce is funding further research to address this problem following on from this research finding.

\subsubsection{Assessing impact}

Impacts of a potential material supply restriction were assessed by taking the cost of raw material inputs and developing an impression of how the price of these materials might change in the future. The basic cost of raw material inputs was relatively easy to find through the use of internal information. Where needed, reliable cost information could also easily be found through internet searches.

Attempting to predict the future prices of raw materials with any degree of certainty is almost impossible. Using price volatility (as calculated in equation 5.4) was deemed to be the best approach. As discussed in the case study, typically these types of problems would be approached using statistical modelling, based on extrapolations of past price trends. This was suggested as an improved approach based on feedback from when an adapted version of the Thames case study was presented as a 
conference paper (Lloyd et al., 2012b). Creating such a model would not have been feasible given the time and resources available to the project. There is also evidence to suggest that taking a more complicated approach that was reliant on extrapolating past trends would not have produced a result that was any more robust, as past prices do not provide a realistic estimate of future trends (Taleb et al., 2009).

\subsubsection{Managing risks from the 'access to resources' hazard}

Purchasing managers within Rolls-Royce already undertake a variety of activities to manage risks similar to those posed by the access to resources hazard, including:

- Using financial instruments such as hedging;

- Vertically aligning supply chains by securing long term supply agreements with primary producers;

- Developing closed loop supply chains, returning material recycled from factories and engine run-parts to primary producers.

From discussions, there is a strong desire within purchasing managers to also influence engineering material selection, potentially avoiding having to take these risk mitigation activities by encouraging designers not to use risky materials. The approach developed here could be used to achieve that aim. Risks associated with materials could be considered by designers, allowing them to be incorporated into design tradeoff decisions. That way designers can make a balanced judgement considering other business priorities, which would be preferable to implementing a blanket ban on the use of some materials, which might have other (and more severe) business consequences. To implement the system the business needs to develop an approach for assessing the probability of a material supply restriction that is more suited to its world view (which it is doing), as well as processes for flagging at risk materials to designers, prompting risk assessment activities.

Problems associated with the use of 'conflict minerals' have come to prominence recently, which is related to the access to resources hazard, as sourcing from conflict areas is a common component of criticality assessments. New interest in this particular aspect of the problem has been engendered by section 1502 of the DoddFrank act (see US Congress, 2010), which imposes requirements for businesses to report on the use of certain materials sourced from the Democratic Republic of the Congo and surrounding regions. Whilst Rolls-Royce will not have to report to the US securities and exchange commission directly, its customers will, requiring Rolls-Royce to identify and report conflict materials contained within the parts it manufactures and 
those that come from suppliers. Dodd-Frank adds the potential for reputational risks to be posed by the access to resources hazard, as well as those associated with cost, as it does not ban conflict materials but requires companies to publicly declare using them. Requirements to fulfil obligations under Dodd-Frank will also require businesses to investigate where the materials of concern are used in products, further highlighting the need for product life cycle environmental data to provide this information.

\subsection{Summary and conclusions}

This chapter has presented case study scenarios testing the framework for environmental risk management using the access to resources hazard identified in chapter 4. Section 5.2 provided more detail on the access to resources hazard, discussing the topic of critical materials that captures the same concerns. A method for identifying critical materials developed by the European Commission (2010) was outlined, providing a list of critical materials, which if found in Rolls-Royce products could be used as the basis of embedded case study risk assessments. Two case studies were defined, the first based on the use of ruthenium in the Thames engine and the second on the use of yttrium in the Severn engine. A risk assessment approach was outlined, adhering as closely as possible to standard risk management practices used within Rolls-Royce. Methods for assessing probability and impact were then defined and applied to produce risk assessment results. The risk calculated for the use of ruthenium on the Thames engine case was significant, requiring mitigating actions. An assessment of the residual risk posed by taking ruthenium out of the engine showed that on balance the material should be removed. Risks posed by the use of yttrium on the Severn engine were shown not to be significant, largely due to the small amount of yttrium used in the component assessed.

Conclusions can be drawn from a discussion of the results and findings provided in section 5.4 :

- Results showed that risks posed by the access to resources hazard could be significant, in particular when at risk materials are used in applications where material costs form a larger fraction of the overall unit cost of parts, for example when used within a part alloy rather than a coating.

- It has been shown how the risk approach allows for environmental considerations to be included in trade-off decisions with other design requirements within design decision-making. This was an important requirement for developing an approach to DfE discussed earlier in this thesis. 
- The need to identify where at risk materials are used within the product system highlights the need for life cycle environmental data within the framework. Data requirements are discussed further in chapter 7.

- Problems with defining risk criteria for the Severn engine case study are indicative of a broader challenge in defining and applying risk criteria for design tasks and the approach taken to define criteria was not a significant deviation from standard risk management practices.

- A bespoke approach for assessing the probability of material supply restrictions is required. Continuing research is being funded by Rolls-Royce to address this problem.

- The approach for assessing the impact of a material supply restriction was sufficiently robust, given the complexity of the problem posed. Feedback from a conference paper published (Lloyd et al. 2012b) suggested that using statistical modelling to extrapolate past price trends would have been a better approach. Resources to do this were not available to the project.

- Purchasing managers already undertake activities to treat risks similar to those posed by the access to resources hazard. It is recognised that a further step is necessary, influencing engineering design decisions to treat risks. The risk assessment method developed here shows how this problem could be approached, allowing designers to balance material supply risks with other design requirements.

- New regulation related to the use of 'conflict minerals', a topic that is related to the access to resources hazard, highlights reputational risks that are also relevant to the business. This final aspect further highlights the importance of managing the access to resources hazard in the future. Supplying customers who have to comply with Dodd-Frank with information on where materials are used in products further highlights the need for product life cycle environmental data. 


\section{Case study scenarios: REACH and energy}

This chapter presents the research activities undertaken to test the framework for environmental risk management using the REACH and energy hazards identified in chapter 4. Research presented in this chapter represents a continuation of the research cycle defined in chapter 5 . As with the case study scenarios presented in chapter 5 , it is necessary to provide more detail on the REACH and energy hazards to enable the selection of the embedded case studies, which is provided in Section 6.1. Section 6.2 defines the case study scenarios and presents assessments of the risk posed by the REACH and energy use hazards to Rolls-Royce products. Section 6.3 discusses the results of the risk assessments and findings. Section 6.4 provides a summary and conclusions for this chapter, leading in to further discussions in chapter 7.

\subsection{REACH and energy}

This section provides more detail on the REACH and energy hazards to help define the embedded case study scenarios. REACH is covered in section 6.1.1; energy in section 6.1.2.

\subsubsection{REACH hazard}

REACH (Registration, Evaluation, Authorisation and Restriction of Chemicals) is a European regulation that aims to improve the protection of human health and the environment from the risks posed by chemicals (see ECHA, 2011a). Coming into law in 2006 (European Commission, 2006), the regulation requires companies to register the use of chemicals through three phases defined by volume of use and the associated environmental hazards ${ }^{4}$ posed, up to 2018 . Uses are then evaluated by the European Chemicals Agency (ECHA) and actions taken to control or restrict the use of chemicals deemed to present a high risk (to the environment) through authorisation or restriction requirements.

As illustrated by the focus group discussions in chapter 4 , of particular concern to businesses is the authorisation requirement under REACH. Authorisation seeks to control the risks posed by particularly harmful substances, categorised as Substances of Very High Concern (SVHCs), through a process of progressive replacement (see

\footnotetext{
${ }^{4}$ In the context of risks posed to the environment, rather than the concept of environmental business hazards as it has been defined in this thesis.
} 
ECHA, 2011b). Once a substance has been placed on annex XIV of REACH (and the sunset date for authorisation has passed), placing it on the market in any form will only be allowed if the regulator grants an authorisation to continue to do so. Whether an authorisation is granted or not is dependent on certain criteria. Any business using SVHCs will be affected, as at some point in the future continued use cannot be guaranteed. It would be prudent for a business to identify its usage of SVHCs and consider what the consequences are if a substance is no longer available.

The probability of a substance becoming unavailable due to authorisation under $\mathrm{REACH}$ is reliant on the regulatory process, specific details of which can be found in the legal text (European Commission, 2006), summarising:

- Article 57 outlines criteria defining classes of SVHCs, any of which may be put on the 'candidate list', which records substances that have been identified as requiring authorisation in the future. Individual EU member states make recommendations for substances to be included on the candidate list on a periodic basis. Recommendations are then evaluated and substances placed on the candidate list, or disregarded.

- Candidate list substances are then prioritised for inclusion on Annex XIV (substances requiring authorisation) through periodic consultations. Criteria for prioritising substances for inclusion on annex XIV are based on their hazardous properties, which are outlined in article 58(3).

- A 'sunset date' is then set, beyond which placing the substance on the market without authorisation is illegal. An application for an authorisation to continue using the substance beyond the sunset date must have been received by the chemicals agency 18 months prior to that date.

From the legal text of REACH and information on the ECHA website (see ECHA, $2011 b$ ), it is clear that authorisation process effectively seeks to phase out the use of substances. An authorisation is only granted subject to a socio-economic analysis of the continued benefits of using a substance in a particular application, balanced against the risk it poses to the environment and human health. A substitution plan is also required outlining how the substance will be replaced. Authorisations are only granted for a fixed period of time, at which point the authorisation is subject to a review and could be withdrawn.

How SVHCs are prioritised towards authorisation from the candidate list is dependent upon open consultation, which makes the process unclear. Only a few substances have been through the whole process and it is unlikely that the same approach will be taken for all chemicals. From discussions with hazardous materials specialists within 
Rolls-Royce, how chemicals are transitioned through from being identified as hazardous to becoming subject to authorisation will be dependent on a balance between risks posed to the environment against risks posed to the economy, which will not be the same in all circumstances. In the context of this research, identifying where SVHCs are used in Rolls-Royce products will allow a risk assessment posed by the REACH hazard.

\subsubsection{Energy use hazard}

Considering the energy use hazard, electricity generation is a significant source of carbon emissions in the UK and a legal commitment has been made to reduce these emissions (Great Britain, Climate Change Act 2008). As illustrated from the focus group discussions presented in chapter 4 , business concerns focus on how the carbon intensity of electricity will lead to increases in its price over time, which is a risk to the business. Regulations to reduce energy use (and associated carbon emissions) through price increases have already been put into place, for example the Climate Change Levy (CCL) and Carbon Reduction Commitment (CRC). Managers within Rolls-Royce are genuinely concerned that this trend will continue, significantly increasing energy costs.

From discussions with energy managers within Rolls-Royce, primary concerns over energy cost increases focus on the impacts this will have on operating overheads. $A$ different perspective is considering how these costs feed through into potential increases in the unit cost of parts, which will impact on engineering design objectives. To investigate this risk, estimates of the energy required to manufacture parts are required together with an evaluation of how the price of energy might change.

\subsection{Case study scenarios}

This section presents the results of the second set of case study scenarios, assessing the risks posed to Rolls-Royce products by the REACH and energy hazards. As in chapter 5 the case studies presented here are pilot studies based on actual product design and business information. Section 6.2.1 presents the first case study identifying the use of zinc chromate in an existing product, demonstrating the framework from the perspective of the dashed arrows (leading from prioritised hazards to in-service products) in Figure 3.15. Zinc chromate is a known carcinogen, which is one of the criteria used to define SVHCs under REACH. Case studies in section 6.2.2 and 
section 6.2.3 are derived from information gathered during a DfE pilot project, completed immediately before this research commenced, demonstrating the framework from the perspective of the solid arrows (leading from design to prioritised hazard) from the framework in Figure 3.15. Section 6.2 .2 assesses risks posed by the use of trichloroethylene in the manufacture of the parts studied, which is also a known carcinogen that is likely to be affected by REACH. Section 6.2.3 uses estimates of the energy used to manufacture the parts to assess the risk posed by the energy hazard.

As in chapter 5 , the risk assessments presented in this chapter were completed by adhering as closely as possible to standard risk management practices within RollsRoyce. The risk assessment approach is presented at the beginning of each case study, structuring the risk using an 'if...then' statement, defining the business risk management criteria and the PID to be used for the risk assessment.

\subsubsection{Zinc chromate in the Severn engine}

An internal Rolls-Royce report (Haneef, 2011) provided an overview of the use of hexavalent chromium compounds in the Severn engine (the same engine that was the subject of the second case study scenario in chapter 5). Being known carcinogens, these compounds are very likely to be affected by $\mathrm{REACH}$ authorisation and are of particular concern to the business. One use identified was the application of a coating primer containing zinc chromate on the oil tank on the Severn engine, used prior to the application of a corrosion resistant coating. At the time of the report, no known alternatives for the zinc chromate primer were known to exist for this application and re-designing the component to use a different coating/primer combination, or with a different base material that did not require corrosion resistance, was judged to be a significant engineering task.

\subsubsection{Risk assessment approach}

The use of zinc chromate in the Severn engine was a concern due to the substance potentially requiring an authorisation under $\mathrm{REACH}$ in the future. If zinc chromate is subject to authorisation it is very likely to be phased-out of use in the future. To capture this concern, the following 'if...then' statement structures the risk assessment that needs to be completed:

\section{If...zinc chromate is placed on annex XIV of the REACH regulation, requiring} authorisation and is phased out of use, 
Then...Rolls-Royce will not be able to manufacture any parts that contain zinc chromate.

Design level risk criteria for the Severn engine was defined in the second case study presented in chapter 5. As this risk assessment is being performed for the same engine the same criteria can be applied and the unit cost risk assessment PID shown in Figure 5.12 will be used for the assessment (Figure 6.1).

\begin{tabular}{|c|c|c|c|c|c|c|}
\hline \multirow{5}{*}{ 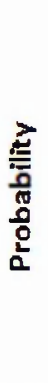 } & $\geq 50 \%$ & 9 & 14 & 19 & 24 & 29 \\
\hline & $\geq 25 \%$ to $<50 \%$ & 7 & 12 & 17 & 22 & 27 \\
\hline & $\geq 5 \%$ to $<25 \%$ & 5 & 10 & 15 & 20 & 25 \\
\hline & $\geq 1 \%$ to $<5 \%$ & 3 & 8 & 13 & 18 & 23 \\
\hline & \multirow[t]{3}{*}{$<1 \%$} & 1 & 6 & 11 & 16 & 21 \\
\hline & & $<f 0.6 k$ & $\begin{array}{l}260.6 k \text { to } s \\
\text { E1k }\end{array}$ & $\begin{array}{c}\geq f 1 k \text { to } \\
<f 5 k\end{array}$ & $\begin{array}{l}\text { 2f5k to } \\
\leqslant f 10 k\end{array}$ & $\geq \mathrm{E} 10 \mathrm{~K}$ \\
\hline & & \multicolumn{5}{|c|}{ Impact } \\
\hline
\end{tabular}

Figure 6.1: PID for Severn engine risk assessment

\subsubsection{Risk assessment}

To perform the risk assessment it is necessary to assess the probability that zinc chromate will be subject to REACH authorisation, eventually becoming unavailable to use, and what the impact will be on the business if this occurs.

To assess the probability of a substance becoming unavailable due to $\mathrm{REACH}$ it is necessary to translate the steps of the authorisation process outlined in section 6.1 to the scale of probability in the PID from Table 6.1. To achieve this, the authorisation process is broken down into the following five steps:

1. Substance is identified as having properties that meet the SVHC criteria defined in article 57;

2. Substance is placed on the candidate list;

3. Substance is prioritised for authorisation;

4. Substance is included on Annex XIV, substances requiring authorisation;

5. Sunset date for authorisation is set, after which a substance cannot be placed on the market without an authorisation. Applications for authorisation must be made 18 months prior to the sunset date.

Each of these steps is assigned a probability score from the PID in Figure 6.1, as shown in Table 6.1. 
Table 6.1: REACH Probability scoring scheme

\begin{tabular}{|c|c|}
\hline Probability (P) & Authorisation step \\
\hline$\geq 50 \%$ & Sunset date set \\
\hline$\geq 25 \%$ to $<50 \%$ & Substance on Annex XIV \\
\hline$\geq 5 \%$ to $<25 \%$ & Prioritised for inclusion on Annex XIV \\
\hline$\geq 1 \%$ to $<5 \%$ & Candidate list \\
\hline$<1 \%$ & SVHC properties \\
\hline
\end{tabular}

At the time of writing zinc chromate was on the REACH candidate list (see ECHA, 2011c), which gives a low (1\% to $5 \%$ ) probability using the scoring scheme from Table 6.1 .

To assess impact, if zinc chromate does become unavailable Rolls-Royce will not be able to manufacture any parts that are dependent on the use of zinc chromate. If the company cannot manufacture the parts that go into an engine, then the engine itself is at risk and the impact on the business, without mitigating actions, is the value of business attached to the engine. Bearing in mind the Severn engine is a relatively old design, from discussions with a financial manager the value of future business at risk was estimated at $£ 72$ million. This is a very high impact using the scale outlined in the PID. The risk assessment can be completed by plotting the scores of probability and impact on the chart given in Table 6.1. With a low probability and an impact of $£ 72$ million the risk assessment score for zinc chromate is 23 , circled in Figure 6.2. A risk of this magnitude would be classed as significant, requiring mitigating actions.

\begin{tabular}{|c|c|c|c|c|c|c|}
\hline \multirow{5}{*}{ 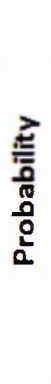 } & $\geq 50 \%$ & 9 & 14 & 19 & 24 & 29 \\
\hline & $\geq 25 \%$ to $<50 \%$ & 7 & 12 & 17 & 22 & 27 \\
\hline & $25 \%$ to $<25 \%$ & 5 & 10 & 15 & 20 & 25 \\
\hline & $\geq 1 \%$ to $<5 \%$ & 3 & 8 & 13 & 18 & 23 \\
\hline & $<1 \%$ & 1 & 6 & 11 & 16 & 21 \\
\hline & & $<E 0.6 \mathrm{k}$ & $\begin{array}{c}\sum E 0.6 \mathrm{klo}< \\
\mathrm{f1k}\end{array}$ & $\begin{array}{c}\sum \notin 1 k \text { io } \\
<\notin 5 k\end{array}$ & $\begin{array}{l}\text { ZESK to } \\
<E 10 k\end{array}$ & 2E10k \\
\hline & & \multicolumn{5}{|c|}{ Impact } \\
\hline
\end{tabular}

Figure 6.2: Risk assessment result

From discussions with a risk manager in Rolls-Royce's defence business, a risk with an impact of this magnitude would be escalated from a design level to be assessed at the level of the engineering function. This is due to there being two to three orders of magnitude difference between the impact of the risk estimated above ( $£ 72$ million) and values in the impact scale of the PID used to assess risk, as shown in Figure 6.2 (a 
significant impact is $\geq £ 10 \mathrm{k}$ ). Risk escalation was discussed as part of the overview of risk management processes in chapter 3 , which described how risks deemed to be very high at one level of the business can be escalated to a higher level, all the way up to the corporate risk register if required. Figure 6.3 illustrates the escalation structure within defence engineering, showing how risks within an individual project are escalated to a central risk management plan for the whole engineering function.

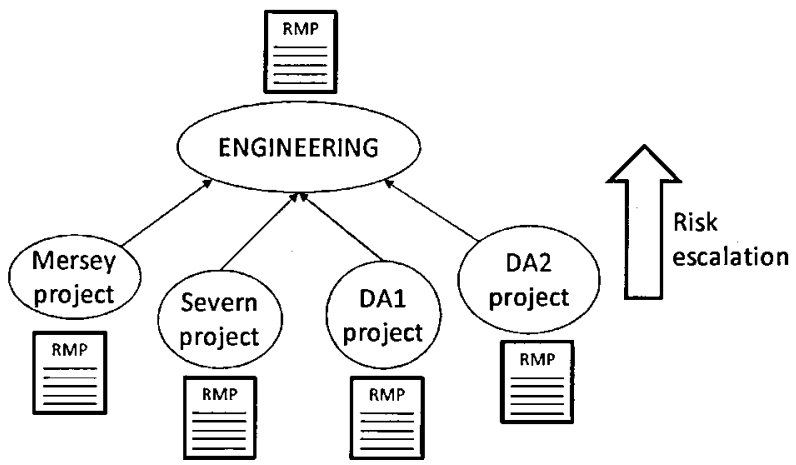

Figure 6.3: Risk management structure in Defence engineering

Risk criteria used at a functional level within engineering are shown in Table 6.2, with impact criteria covering the defence business' ten year profit forecast, changes to programme time, changes to product specifications and effects on business reputation.

Table 6.2: Engineering function risk criteria (Snape, 2009)

\begin{tabular}{|c|c|c|c|c|c|}
\hline \multirow{2}{*}{ Category } & \multirow{2}{*}{ Probability } & \multicolumn{4}{|c|}{ Impact } \\
\hline & & Time & ENPV* & Specification & Reputation \\
\hline Very Low & $\leq 1 \%$ & $\begin{array}{l}<4 \text { week slip } \\
\text { to plan }\end{array}$ & $<£ 0.5 m$ & $\begin{array}{l}\text { Minimal change } \\
\text { to specification }\end{array}$ & $\begin{array}{l}\text { Minimal damage to } \\
\text { stakeholder trust, } \\
\text { could be easily } \\
\text { recovered. Risk could } \\
\text { be contained at the } \\
\text { local operational level }\end{array}$ \\
\hline Low & $>1 \%$ to $\leq 5 \%$ & $\begin{array}{l}4 \text { to } 8 \text { week } \\
\text { slip to plan }\end{array}$ & $\begin{array}{l}\geq £ 0.5 \mathrm{~m} \\
\text { to }<£ 2 \mathrm{~m}\end{array}$ & $\begin{array}{l}\text { Minor change to } \\
\text { specification }\end{array}$ & $\begin{array}{l}\text { Stakeholder trust } \\
\text { dented, could be } \\
\text { recovered. Risk could } \\
\text { be contained at the } \\
\text { business level }\end{array}$ \\
\hline Medium & $\begin{array}{c}>5 \% \text { to } \leq \\
25 \%\end{array}$ & $\begin{array}{l}8 \text { to } 16 \text { week } \\
\text { slip to plan }\end{array}$ & $\begin{array}{l}\geq £ 2 m \text { to } \\
<£ 10 m\end{array}$ & $\begin{array}{l}\text { Moderate } \\
\text { change to } \\
\text { specification }\end{array}$ & $\begin{array}{l}\text { Stakeholder trust } \\
\text { damaged, recovery } \\
\text { could be difficult. Risk } \\
\text { could be contained at } \\
\text { the sector level }\end{array}$ \\
\hline High & $\begin{array}{l}>25 \% \text { to } \\
\leq 50 \%\end{array}$ & $\begin{array}{l}16 \text { to } 52 \text { week } \\
\text { slip to plan }\end{array}$ & $\begin{aligned} & \geq £ 10 \mathrm{~m} \text { to } \\
&<£ 50 \mathrm{~m}\end{aligned}$ & $\begin{array}{l}\text { Major change to } \\
\text { specification }\end{array}$ & $\begin{array}{l}\text { Stakeholder trust } \\
\text { severely damaged, } \\
\text { recovery could be } \\
\text { extremely difficult. } \\
\text { Risk would involve } \\
\text { attention of the group } \\
\text { executive. }\end{array}$ \\
\hline Very High & $>50 \%$ & $\begin{array}{c}>52 \text { week slip } \\
\text { to plan }\end{array}$ & $\geq £ 50 \mathrm{~m}$ & $\begin{array}{l}\text { Fundamental } \\
\text { change to } \\
\text { specification }\end{array}$ & $\begin{array}{l}\text { Stakeholder trust } \\
\text { completely lost, full } \\
\text { recovery would be } \\
\text { questionable. Risk } \\
\text { would involve the } \\
\text { attention of Chief } \\
\text { Executive }\end{array}$ \\
\hline
\end{tabular}

*Values expressed in NPV are discounted at $10 \%$ p.a. over the TYF 
Focusing on impact to business revenue, and transferring the risk criteria from Table 6.2 into a standard risk PID, Figure 6.4 shows the risk assessment result for the use of zinc chromate on the Severn engine when assessed at an engineering functional level.

\begin{tabular}{|c|c|c|c|c|c|c|}
\hline \multirow{5}{*}{ 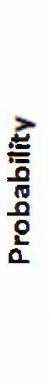 } & $250 \%$ & 9 & 14 & 19 & 24 & 29 \\
\hline & $\geq 25 \%$ to $<50 \%$ & 7 & 12 & 17 & 22 & 27 \\
\hline & $25 \%$ to $<25 \%$ & 5 & 10 & 15 & 20 & 25 \\
\hline & $\geq 1 \%$ to $<5 \%$ & 3 & 8 & 13 & 18 & 23 \\
\hline & $<1 \%$ & 1 & 6 & 11 & 16 & 21 \\
\hline & & $<f 0.5 \mathrm{~m}$ & $\begin{array}{l}\geq \mathrm{f} 0.5 \mathrm{~m} \text { to }< \\
\mathrm{f} 2 \mathrm{~m}\end{array}$ & $\begin{array}{l}\text { ef2m to } \\
<f 10 m\end{array}$ & $\begin{array}{l}\text { ef10m to } \\
<\mathrm{f50m}\end{array}$ & $2 f 50 \mathrm{~m}$ \\
\hline & & & & Impact & & \\
\hline
\end{tabular}

Figure 6.4: Risk assessment result using engineering function risk criteria

With a risk assessment result of 23 the risk will still be classed as significant, requiring treatment actions. However, escalating the risk has produced a result where the scale of impact is more appropriate.

\subsubsection{Sensitivity analysis}

As discussed, the regulatory process that controls whether or not a substance may become unavailable due to REACH is rather opaque, creating uncertainties within the assessment of probability in particular. Given knowledge of the regulatory process is critical to the assessment of probability, rather than using the scale from Table 6.1, a more qualitative assessment dependant on expert judgement might be more appropriate. A restricted substances specialist within Rolls-Royce was given the task of defining, on the scale given in Table 6.1, the probability that zinc chromate might become unavailable due to REACH. Their assessment was that it is almost inevitable that at some point in the future zinc chromate will become restricted due to $\mathrm{REACH}$. In the short term (say 3 years, a frequently used planning horizon for Rolls-Royce) the probability of zinc chromate becoming unavailable may still be low. Although expand the time horizon (say to 30 years, the expected in-service life of a product) and the result will escalate up the $y$-axis of the PID.

There are fewer sensitivities in the measure of impact; if a product cannot be manufactured the impact is always going to be very large, as technically the engine cannot be made and all business related to that engine is at risk. As presented in the risk assessment, large risks are typically escalated to the appropriate level within the 
business. Risk managers within Rolls-Royce recommended assessing these types of risks at the level of the engineering function.

\subsubsection{Treatment actions and residual risks}

There are three options to treat the risks associated with the use of zinc chromate on the Severn engine:

- Use an alternative primer;

- Use an alternative coating that either does not need a primer or uses a primer not at risk of substance restrictions;

- Re-design the component with a base metal that does not require corrosion protection.

Discussions with a hazardous materials specialist within Rolls-Royce confirmed that, at the time of writing there are no known alternatives to zinc chromate in this application. The engine project would have to consider re-designing the fuel tank with a different base material to mitigate the risk, which is a fundamental change to the engine, to treat the risk. Residual risks posed can be assessed by using the change to specification impact ratings from Table 6.2 (p.168, column 5). Creating a PID with these impact to specification ratings on the $x$-axis, and taking a change of the fuel tank base material as a fundamental impact, produces the residual risk assessment result presented in Figure 6.5. The probability rating in Figure 6.5 is taken from the previous risk assessment result (Figure 6.4), as this impact will only be incurred if zinc chromate becomes unavailable.

\begin{tabular}{|c|c|c|c|c|c|c|}
\hline \multirow{5}{*}{ 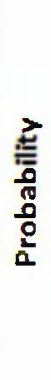 } & $250 \%$ & 9 & 14 & 19 & 24 & 29 \\
\hline & $225 \%$ to $<50 \%$ & 7 & 12 & 17 & 22 & 27 \\
\hline & $25 \%$ to $<25 \%$ & 5 & 10 & 15 & 20 & 25 \\
\hline & $21 \%$ to $<5 \%$ & 3 & 8 & 13 & 18 & 23 \\
\hline & \multirow[t]{3}{*}{$<1 \%$} & 1 & 6 & 11 & 16 & 21 \\
\hline & & Minimal & Minor & Moderate & Major & Fundamental \\
\hline & & \multicolumn{5}{|c|}{ Impact to specification } \\
\hline
\end{tabular}

Figure 6.5: Residual risk assessment result 


\subsubsection{Summary}

This section has presented a case study scenario showing how the framework can be applied to assess the risks posed to an in-service product from $\mathrm{REACH}$. The risk assessment showed that the risk posed to business objectives by using zinc chromate on the fuel tank of the Severn engine is significant, even when escalated to be assessed at an engineering function level. Residual risks related to treatment actions required to mitigate the risk were also assessed to be significant.

\subsubsection{Trichloroethylene on the Mersey engine}

Immediately before this research began, a DfE study was completed within RollsRoyce's Defence business that collected life cycle environmental data for the materials and manufacturing processes used to manufacture a compressor stage blisk on one of the business' newest low-bypass ratio turbofan engines. The methodology used to gather the life cycle environmental data was similar to the one described in chapter 1. For reasons of commercial sensitivity this engine will be referred to as the 'Mersey' engine. A $3 \mathrm{D}$ image of the Mersey engine module containing the blisk studied is shown in Figure 6.6.

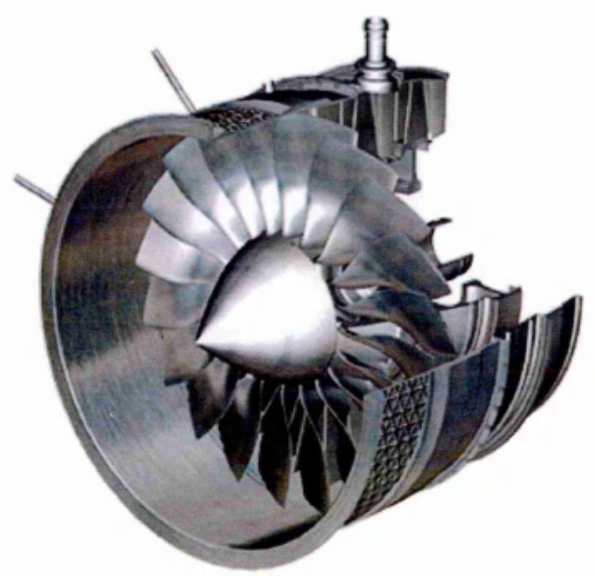

Figure 6.6: Mersey engine low-pressure compressor module (Rolls-Royce, 2007a)

A blisk consists of a series of compressor blades welded to a compressor disc, forming one rotating stage of the Mersey engine's low pressure compressor. The blisk assembly is welded to other low pressure compressor stages and connected to the low pressure shaft. The blisk is manufactured from titanium alloy and is roughly $400 \mathrm{~mm}$ in diameter when finished, weighing approximately $35 \mathrm{~kg}$. From an analysis of the manufacturing processes used to make the blisk, it was identified that trichloroethylene 
is used as a degreaser to clean parts between manufacturing operations. Being a carcinogen, trichloroethylene meets one of the REACH SVHC criteria.

\subsubsection{Risk assessment approach}

The use of trichloroethylene during the manufacture of the Mersey engine was a concern due to the substance potentially requiring an authorisation under $\mathrm{REACH}$. If trichloroethylene is subject to authorisation it is very likely to be phased-out of use in the future. To capture this concern, the following 'if...then' statement structures the risk assessment that needs to be completed:

\section{If... Trichloroethylene is placed on annex $X I V$ of the $R E A C H$ regulation, requiring authorisation and eventual phase out of use,}

Then...Rolls-Royce will not be able to manufacture any parts dependant on processes that use trichloroethylene.

Managers within Rolls-Royce have suggested that risks posed by REACH should be assessed at the level of the engineering function, following on from the results of the previous case study, which highlighted a large difference between the magnitude of the potential impact of the risk and the risk impact criteria used at an engineering design level. Risk criteria for the engineering function were provided in Table 6.2. A risk scoring PID for this assessment is provided in Figure 6.7, using criteria for assessing impacts to business revenue.

\begin{tabular}{|c|c|c|c|c|c|c|}
\hline \multirow{5}{*}{ 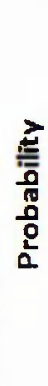 } & $\geq 50 \%$ & 9 & 14 & 19 & 24 & 29 \\
\hline & $\geq 25 \%$ to $<50 \%$ & 7 & 12 & 17 & 22 & 27 \\
\hline & $25 \%$ to $<25 \%$ & 5 & 10 & 15 & 20 & 25 \\
\hline & $21 \%$ to $<5 \%$ & 3 & 8 & 13 & 18 & 23 \\
\hline & $<1 \%$ & 1 & 6 & 11 & 16 & 21 \\
\hline & & $<f 0.5 \mathrm{~m}$ & $\begin{array}{l}2 f 0.5 \mathrm{~m} \text { to } \\
\mathrm{f2} 2 \mathrm{~m}\end{array}$ & $\begin{array}{l}2 \mathrm{f} 2 \mathrm{~m} \text { to } \\
<\mathrm{E} 10 \mathrm{~m}\end{array}$ & $\begin{array}{c}\geq f 10 \mathrm{~m} \text { to } \\
<£ 50 \mathrm{~m}\end{array}$ & $\geq \mp 50 \mathrm{~m}$ \\
\hline
\end{tabular}

Figure 6.7: Risk assessment PID (Rolls-Royce, 2009) 


\subsubsection{Risk assessment}

To perform the risk assessment it is necessary to assess the probability that trichloroethylene will be subject to REACH authorisation, eventually becoming unavailable to use, and the impact this will have on the business if this occurs.

A method for assessing the probability of a substance becoming unavailable due to $\mathrm{REACH}$ authorisation was developed in the previous case study. Steps of the authorisation process were aligned with the scale of probability from a typical PID, producing the scoring scheme presented in Table 6.1. From ECHA (2011c), trichloroethylene is on the REACH candidate list and has been prioritised for authorisation, although no sunset date has been set, producing a probability score of medium ( $5 \%$ to $25 \%$ ).

As in the previous case study, if trichloroethylene does become unavailable RollsRoyce will not be able to manufacture any parts that are dependent on processes that use trichloroethylene. If the company cannot manufacture the parts that go into an engine, then the engine itself is also at risk. The impact on the business, without mitigating actions, is the value of business that is predicted to come from selling the engine in the future.

From discussions with a financial manager within Rolls-Royce, the value of future business expected to come from the Mersey engine, taken from a business profit forecast for ten years, is approximately $£ 2$ billion. With a figure of this order of magnitude a great degree of precision is not required. It is clear that the impact is very high ( $>£ 50$ million) using the scoring scheme outlined in Figure 6.7.

The risk assessment can be completed by plotting the scores of probability and impact on the chart given in Figure 6.7. With a medium probability and a very high impact (greater than $£ 50$ million) the risk assessment score is 25 , circled in Figure 6.8. A risk of this magnitude would be classed as significant, requiring mitigating actions. 


\begin{tabular}{|c|c|c|c|c|c|c|}
\hline \multirow{5}{*}{ 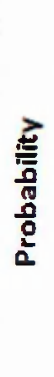 } & $\geq 50 \%$ & 9 & 14 & 19 & 24 & 29 \\
\hline & $\geq 25 \%$ to $<50 \%$ & 7 & 12 & 17 & 22 & 27 \\
\hline & $25 \%$ to $<25 \%$ & 5 & 10 & 15 & 20 & 25 \\
\hline & $\geq 1 \%$ to $<5 \%$ & 3 & 8 & 13 & 18 & 23 \\
\hline & \multirow[t]{3}{*}{$<1 \%$} & 1 & 6 & 11 & 16 & 21 \\
\hline & & $<f 0.5 m$ & $\begin{array}{l}\geq £ 0.5 m \text { to }< \\
\qquad 2 \mathrm{~m}\end{array}$ & $\begin{array}{l}2 £ 2 m \text { to } \\
<\notin 10 m\end{array}$ & $\begin{array}{l}\text { ef10m to } \\
<\mathrm{f} 50 \mathrm{~m}\end{array}$ & $\geq\{50 \mathrm{~m}$ \\
\hline & & \multicolumn{5}{|c|}{ Impact } \\
\hline
\end{tabular}

Figure 6.8: Risk assessment result

\subsubsection{Sensitivity analysis}

A more qualitative assessment of probability can be used to assess the sensitivity of the result, as discussed in the previous case study. Using expert judgements from within the business, it was assessed that the probability of trichloroethylene becoming unavailable due to REACH was low ( $1 \%$ to $5 \%$, using the scale defined in Table 6.1), which is less than the previous assessment and would produce a risk score of 23 (Figure 6.9). The specialist argued a lower score was justified as trichloroethylene is generally used in a fully contained system, minimising risks to the environment. However their judgement did go with the caveat that these decisions appeared to be made "at the whim of the regulator".

\begin{tabular}{|c|c|c|c|c|c|c|}
\hline \multirow{5}{*}{$\begin{array}{l}\frac{2}{2} \\
\frac{5}{0} \\
0 \\
0 \\
0 \\
0\end{array}$} & $250 \%$ & 9 & 14 & 19 & 24 & 29 \\
\hline & $225 \%$ to $<50 \%$ & 7 & 12 & 17 & 22 & 27 \\
\hline & $25 \%$ to $<25 \%$ & 5 & 10 & 15 & 20 & 25 \\
\hline & $\geq 1 \%$ to $<5 \%$ & 3 & 8 & 13 & 18 & 23 \\
\hline & \multirow[t]{3}{*}{$<1 \%$} & 1 & 6 & 11 & 16 & 21 \\
\hline & & $<\notin 0.5 m$ & $\begin{array}{l}\geq f 0.5 m \text { to }< \\
f 2 m\end{array}$ & $\begin{array}{l}\geq f 2 m \text { to } \\
<f 10 m\end{array}$ & $\begin{array}{l}\geq f 10 \mathrm{~m} \text { to } \\
<\mathrm{f} 50 \mathrm{~m}\end{array}$ & $\geq £ 50 \mathrm{~m}$ \\
\hline & & \multicolumn{5}{|c|}{ Impact } \\
\hline
\end{tabular}

Figure 6.9: Risk assessment result with alternative probability

Further discussions indicated that there was a time dependency to the probability assessment for REACH. Due to the carcinogenic properties of trichloroethylene, its widespread use, and the availability of alternatives, it was judged that in the long term, it is very likely (almost certain) to become unavailable. As in the previous case study, if 
the time horizon is expanded the risk assessment result will escalate up the $y$-axis of the PID.

There are fewer sensitivities in the measure of impact; if a product cannot be manufactured the impact is always going to be very large. The impact of the risk was very large mainly due to the fact that the Mersey engine is a relatively new product and there is estimated to be a lot of potential future business made from selling it. In reality a risk with an impact of this magnitude would be escalated to the Rolls-Royce corporate risk register (REACH in general is already on there). What this risk assessment highlights is the importance of mitigating the risks posed by the use of trichloroethylene.

\subsubsection{Treatment actions and residual risks}

The most pro-active approach to dealing with the risks posed by the use of trichloroethylene would be to encourage designers to engage with manufacturing engineers to ensure that the processing route for their parts did not involve a process that uses the substance. However, given that trichloroethylene is widely used, and degreasing is a common operation in manufacturing routes, this may not be feasible. Assessing the risks posed by REACH would highlight these problems to the engineering design team so an alternative route could be investigated.

The next obvious solution would be to replace the use of trichloroethylene with a different degreaser, although this option has some caveats. It must be ensured that any alternative degreaser does not have SVHC properties, as this would mean that it will also become a similar risk in the future due to REACH. Understanding the engineering and business implications of changing the degreaser must also be understood, in particular if using an alternative affects the product in some way. It may be that, for example, the new degreaser is more expensive, or less effective, which would increase processing time. If the processing route is more expensive this may impact on unit cost, which could create a residual risk. It may be the case that research activity to validate new processing routes or degreasing alternatives is required, independent of the engineering design team. Conducting a proper risk assessment should help to justify the business case for doing so.

Whatever mitigating actions are taken, these will quickly reduce what is a very large risk for the business (of the order of millions of pounds) into one that is relatively small (of the order of thousands). Residual risks would be associated with altering the manufacturing route to avoid the use of trichloroethylene. This would require 
appropriate engineering drawing changes to reflect the new manufacturing specifications that would be used, being classed as a minimal change to specification (using the criteria from Table 6.2, column 5). A residual risk PID reflecting the need for a minimal specification change is presented in Figure 6.10 .

\begin{tabular}{|c|c|c|c|c|c|c|}
\hline \multirow{5}{*}{ 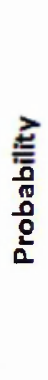 } & $250 \%$ & 9 & 14 & 19 & 24 & 29 \\
\hline & $225 \%$ to $<50 \%$ & 7 & 12 & 17 & 22 & 27 \\
\hline & $25 \%$ to $<25 \%$ & 5 & 10 & 15 & 20 & 25 \\
\hline & $\geq 1 \%$ to $<5 \%$ & 3 & 8 & 13 & 18 & 23 \\
\hline & \multirow[t]{3}{*}{$<1 \%$} & 1 & 6 & 11 & 16 & 21 \\
\hline & & Minimal & Minor & Moderate & Major & Fundamenta \\
\hline & & \multicolumn{5}{|c|}{ Impact } \\
\hline
\end{tabular}

Figure 6.10: Residual risk assessment result

\subsubsection{Summary}

This section has presented a case study scenario, showing how the framework can be used to assess the risks posed to a new design from REACH. Risks posed by the use of trichloroethylene were shown to be significant, although mitigating actions reduced a very large risk to one that was relatively small. In the end it was judged that in the longer term the probability of trichloroethylene becoming unavailable was a near certainty and that designing an engine with a manufacturing route that used trichloroethylene would end up requiring a minimal change to specification. The next case study assesses the risks posed to the same design from the energy hazard.

\subsubsection{Energy use and the Mersey engine}

The final case study focuses on assessing the risks posed by the energy use hazard. Data on the energy used to manufacture the Mersey engine blisk was collected as part of the DfE study discussed previously. Estimates of energy required to manufacture the Mersey blisk were used to assess how potential increases in energy prices may impact on engineering design objectives. 


\subsubsection{Risk assessment approach}

The aim of this risk assessment is to explore how carbon emissions from electricity generation may lead to potential increases in energy prices, feeding through to increases in the unit cost of parts, which will impact on engineering design objectives. Capturing these concerns, the following 'if...then' statement is used to structure the risk assessment:

If...the price of carbon is reflected in energy prices, causing them to increase,

Then...Rolls-Royce will have to pay more for its electricity, increasing the costs of manufacturing parts.

Risk criteria for the Mersey engine are the same as that for the Severn engine, being a similar, albeit newer, low-bypass ratio defence turbofan. Focusing on impact to unit cost, Figure 6.11 presents the risk assessment PID that will be used for the risk assessment.

\begin{tabular}{|c|c|c|c|c|c|c|}
\hline \multirow{5}{*}{ 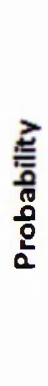 } & $250 \%$ & 9 & 14 & 19 & 24 & 29 \\
\hline & $\geq 25 \%$ to $<50 \%$ & 7 & 12 & 17 & 22 & 27 \\
\hline & $\geq 5 \%$ to $<25 \%$ & 5 & 10 & 15 & 20 & 25 \\
\hline & $\geq 1 \%$ to $<5 \%$ & 3 & 8 & 13 & 18 & 23 \\
\hline & $<1 \%$ & 1 & 6 & 11 & 16 & 21 \\
\hline & & $<€ 0.6 \mathrm{k}$ & $\begin{array}{c}\geq f 0.6 k \text { to }< \\
\text { f1k }\end{array}$ & $\begin{array}{l}\geq f 1 k \text { to } \\
<E 5 k\end{array}$ & $\begin{array}{l}\geq £ 5 k \text { to } \\
<\in 10 k\end{array}$ & $\geq f 10 k$ \\
\hline & & & & Impact & & \\
\hline
\end{tabular}

Figure 6.11: Risk assessment PID

\subsubsection{Risk assessment}

To perform the risk assessment it is necessary to assess the probability that carbon emissions from energy generation will lead to increases in prices and the impact this will have on the business if this occurs.

Energy managers within Rolis-Royce were consulted to provide an assessment of how likely it is that energy prices will increase. It was judged that it was very likely that energy prices will increase due to regulatory measures that seek to reduce carbon emissions. This judgement was based on the fact that several measures to reflect the cost of carbon in energy prices are already in place, for example the Carbon Reduction 
Commitment ( $\mathrm{CRC}$ ), and given targets to reduce carbon emissions have been put into law through the Climate Change Act (Great Britain, 2008), this trend is likely to continue. However, whilst it was judged to be very likely that energy prices will increase in future, the challenge was assessing by how much. Initially the CRC sets a carbon price of $£ 12 /$ tonne $\mathrm{CO}_{2}$, although within phase II of the EU ETS (Emissions Trading Scheme) the carbon price peaked at almost $£ 26 /$ tonneCO $\mathrm{CO}_{2}(\mathrm{HMRC}, 2010)$.

A means of estimating future prices of energy is required to assess impact. Electricity prices generally fluctuate over time. Figure 6.12 shows how electricity prices have changed, in real terms, since 1970. How are further measures that seek to reduce carbon emissions from electricity use likely to increase prices?

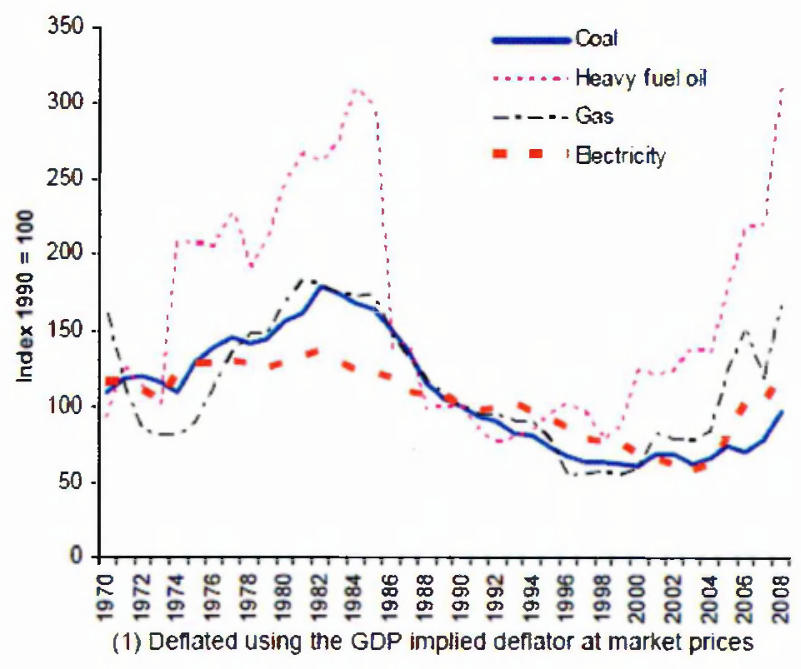

Figure 6.12: Historical energy price trends (DECC, 2009)

There are several ways in which the problem could be approached. One option would be to anticipate further increases in the price of electricity linked to the price of carbon. Another approach could be to extrapolate price trends from Figure 6.12 into the future, given electricity prices have been increasing significantly anyway, although problems with extrapolating past prices to predict the future were discussed in chapter 5 .

Given data on the price of carbon is readily available, the first approach is taken. To estimate the impact it is necessary to settle on a price of carbon and analyse how this would impact on business objectives. Taking a worst case scenario, the peak carbon price from the EU ETS of $£ 26 /$ tonneCO $\mathrm{C}_{2}$ will be used. This fits well with the estimated price required to meet an emissions reduction target of $30 \%$ by 2020 (compared to 1990) within the EU (see HMRC, 2010).

To calculate the impact on the business at a product level, resulting from an increase in electricity prices, it is necessary to identify:

1. The amount of electricity to manufacture a part; 
2. Estimates of the amount of carbon created from this energy use;

3. How much this carbon costs and how this impacts on cost objectives.

An analysis on the parts for this case study is presented in Table 6.3. The amount of electricity required to manufacture the blisk was provided by life cycle environmental data. Data on the cost of electricity and carbon intensity of UK grid electricity was taken from UK government statistics. The overall change in unit cost due to an increase in electricity prices is estimated to be $£ 300$.

Table 6.3: Electricity cost estimates

\begin{tabular}{|l|c|l|}
\hline \multicolumn{1}{|c|}{ Variable (units) } & Value & \multicolumn{1}{c|}{ Source } \\
\hline Electricity (MJ) & 85498.6 & From life cycle environmental data \\
\hline $\mathrm{CO}_{2}$ (tonnes) & 11.54 & $135 \mathrm{gCO}_{2} / \mathrm{MJ}(\mathrm{HMRC}, 2010)$ \\
\hline Cost & $£ 300.54$ & $£ 26 /$ tonne $\mathrm{CO}_{2}$ \\
\hline Old electricity cost & $£ 1662.47$ & $7 \mathrm{p} / \mathrm{kWh}(\mathrm{DECC}, 2010)$ \\
\hline New electricity cost & $£ 1962.57$ & \\
\hline
\end{tabular}

The risk assessment can be completed by plotting the scores of probability and impact on the chart given in Figure 6.11. With a very high probability and a very low impact the risk assessment score is 9 (Figure 6.13). A risk of this magnitude would not require mitigating actions.

\begin{tabular}{|c|c|c|c|c|c|c|}
\hline \multirow{5}{*}{$\begin{array}{l}\frac{2}{0} \\
\frac{5}{0} \\
\frac{0}{0} \\
0 \\
0 \\
\frac{2}{2}\end{array}$} & $250 \%$ & 9 & 14 & 19 & 24 & 29 \\
\hline & $225 \%$ to $<50 \%$ & 7 & 12 & 17 & 22 & 27 \\
\hline & $25 \%$ to $<25 \%$ & 5 & 10 & 15 & 20 & 25 \\
\hline & $\geq 1 \%$ to $<5 \%$ & 3 & 8 & 13 & 18 & 23 \\
\hline & $<1 \%$ & 1 & 6 & 11 & 16 & 21 \\
\hline & & $<\mathrm{E0.6k}$ & $\begin{array}{c}\geq \mathrm{f0} 0.6 \mathrm{k} \text { to } \\
\mathrm{f} 1 \mathrm{k}\end{array}$ & $\begin{array}{c}\geq £ 1 k \text { to } \\
<\notin 5 k\end{array}$ & $\begin{array}{l}\geq f 5 k \text { to } \\
<f 10 k\end{array}$ & 2f10k \\
\hline & & \multicolumn{5}{|c|}{ Impact } \\
\hline
\end{tabular}

Figure 6.13: Risk assessment result

\subsubsection{Sensitivity analysis}

Risks posed to engineering unit cost objectives have been assessed as very low, as estimated increases in the cost of electricity are small relative to the total cost. There are significant uncertainties associated with the future price of carbon. Instead of attempting to predict future prices, an alternative way to look at the problem is to take a 
price of carbon at which impacts on costs would be significant and to assess how likely it is that the price will reach this level.

Working backwards from the scale of impact presented in Figure 6.11, to be classed as significant the impact on unit cost would have to be over $£ 1000$. Using the figures from Table 6.3, the amount of carbon emitted from manufacturing the part was approximately 11.5 tonnes (this seems like a large figure, although the component requires over 120 hours of machining). For this carbon to be worth $£ 1000$, the carbon price needs to be approximately $£ 87 /$ tonne $\mathrm{CO}_{2}$. From HMRC (2010), the highest carbon price up to 2030 used in any scenario was $£ 70 /$ tonneCO $\mathrm{CO}_{2}$. It is unlikely that risks to product cost through increases in electricity prices, due to the added costs of carbon, will be significant for the foreseeable future, based on these figures.

\subsubsection{Summary}

This section has presented a case study scenario, showing how the framework can be used to assess the risks posed to a new design from the energy hazard. Risks posed were shown to be insignificant and it was shown how future increases in prices are also unlikely to significantly impact on product costs.

\subsection{Discussion}

This section discusses the results of the case studies and findings. To provide structure, discussions of results and findings are separated into the following topics:

- Results of the risk assessments;

- Risk assessment approach, including the need for life cycle data within the framework;

- Methods for assessing probability and impact.

Section 6.4.4 summarises discussions with recommendations for managing risks posed by the REACH and energy hazards.

\subsubsection{Results}

Results from the first two case studies showed that the risks posed by REACH are significant. Discussing results of the first risk assessment with risk managers within Rolls-Royce led to an escalation of the risk from design level risk management to 
management at the engineering functional level (which sits one level below risk management for the Defence CFBU). At this level the risks posed were still deemed to be significant. In reality the magnitude of the second risk assessment would require escalation to the corporate risk register, which gets attention from the board.

A scenario where a substance becoming unavailable due to $\mathrm{REACH}$ leads to engine orders being unfulfilled should never become a reality, as the business is going to take whatever measures necessary to make sure it can continue manufacturing products. Assessing the magnitude of the residual risks is likely to provide a more accurate picture of the potential impact on the business and the two case studies provide an interesting insight. Residual risks posed by the use of zinc chromate were significant as the substance is used within engine components and no known alternatives are currently available. Re-designing the oil tank to remove the substance from the engine will be a significant undertaking, requiring significant engineering effort (and hence time and cost). As trichloroethylene is only used in manufacturing processes the residual risks were much less significant and in a worst case a minor specification change will be needed. This finding suggests that the business should focus on identifying where at risk substances are used in products for which there are no alternatives available.

This finding also shows the importance of highlighting the risks posed by substances that are going to be affected by REACH to design teams. This problem was discussed with a designer who was considering designing a shaft on one of Rolls-Royce's newest products that would require a hexavalent chromium corrosion resistant coating. At the time there was no knowledge of the risks posed by REACH. Designing the shaft from a different base metal, for example nickel instead of steel, would remove the need for corrosion resistance. Such a decision may affect other engineering design objectives, such as weight. However, considering the risks posed would at least allow for a judgement to be made on whether or not the weight benefits of using a steel shaft justify the risks of relying on there being a suitable alternative to hexavalent chromium coatings when they eventually become unavailable, and undertaking the effort to redesign the shaft with a new coating. Potentially this may only lead to a minor specification change. Worst case the whole shaft would have to be re-designed and the engine would have to be re-certified for airworthiness. Highlighting the risk at least allows designers to consider the best course of action, bearing in mind all potential consequences.

The results of the risk assessment do confirm concerns related to hexavalent chromium compounds in particular, and REACH more broadly, from the focus group discussions in chapter 4 . It is clear why REACH came out as the biggest issue by 
some margin. The magnitude of the risks assessed highlights the importance of developing alternatives to substances that are going to be affected by $\mathrm{REACH}$, as well as businesses understanding their potential exposure by identifying where at risk substances are used in products.

Findings from the third risk assessment for electricity hazard produced an interesting conclusion. Previous discussions with environmental managers within Rolls-Royce suggested that operational energy use was a significant problem, particularly in the future due to increases in prices anticipated as a result of efforts to reduce carbon emissions. Through the sensitivity analysis it was shown that, at least when assessing how increases of electricity prices may impact product costs, it is unlikely that prices will reach a significant level at any point in the foreseeable future. This result is particularly interesting as, relative to other components, the blisk is quite an energy intensive part to manufacture. If energy costs are not likely to be significant for this part, then perhaps it is less likely they will be for others too. However, this result does not necessarily mean the electricity use hazard can be overlooked. Requirements for managing the energy hazard are discussed further in section 6.3.4.

\subsubsection{Risk assessment approach}

As in chapter 5 , both case studies were scenarios, although actual data from within Rolls-Royce was used. Both engines are well established designs. The Mersey engine case studies used data collected for the DfE study and the scenario was conducted as if the blisk was being designed as new. The first case study assessing risks posed by the use of zinc chromate on the Severn engine is a live issue that needs to be managed. The only factor that makes it a pilot study is that the risk assessment was not performed as part of a business decision. REACH is on the Defence business' engineering function risk register, although as a general item that has not been properly assessed in terms of probability and impact. Risk managers were keen to see the risk quantified to a better degree and did not argue with the result provided. The focus then shifted to understanding the actions required to mitigate the risks and the residual risks associated with those actions, although as the specific actions required remain unknown, and no know alternatives exist, the worst case scenario of changing the oil tank base material was used for the residual risk assessment.

The first case study was identified through a previous analysis of where hexavalent chrome compounds were used on the Severn engine. The second and third case studies were identified through pre-existing life cycle environmental data. As discussed 
in the previous chapter, if the framework were to be implemented into the business life cycle environmental data would be required to identify where SVHCs were used in products and where new designs incorporated the use of SVHCs.

Defining risk criteria for the case studies in this chapter was less problematical. Criteria defined for the Severn engine was defined in chapter 5 and this could be read straight across to the Mersey engine. Engineering function risk criteria were readily available from engineering managers.

\subsubsection{Assessing probability and impact}

Developing a method for assessing the probability of a substance being affected by $\mathrm{REACH}$ was relatively straight forward. Steps within the authorisation process were readily identifiable and these could be read-across directly to the scale of probability within a standard risk PID. The business is currently developing a similar risk rating process for highlighting at risk substances that engineering teams need to be aware of, which is likely to be implemented through a design standard.

As discussed, the reality of how substances progress through this process is unclear, being dependent on consultations that balance risks posed to the environment with the economic benefits of continuing to use a substance. Consulting experts to give their views of how likely a substance may become unavailable may be a more reliable process for assessing probability. The business is currently developing an approach for rating the 'riskiness' of a substances that will be affected by REACH and this approach does include an element of judgement from hazardous materials specialists.

Assessing impact was relatively straight forward; as if a substance becomes unavailable the consequences are clear. The value of presenting such a large impact was questioned and, as discussed, a more productive approach may be to focus on the risks associated with mitigating actions, as this may provide a more accurate reflection of the likely impact on the business. As in the case of zinc chromate, highlighting the need for fundamental design changes should encourage the support of activities to develop alternatives.

For the energy hazard, expert judgement was used for the assessment of probability and there does not appear to be a way of doing this more deterministically. As with attempting to predict the future prices of materials for the access to resources hazard, attempting to predict future energy prices is an almost impossible task. Given regulatory concerns focus on the need to reduce energy use because of the carbon 
associated with generation, it was interesting to analyse how factoring the price of carbon in to energy costs may impact on the costs of manufacturing parts.

\subsubsection{Managing risks posed by the REACH and energy hazards}

It is clear that risks posed by the REACH hazard are significant and concerns expressed during the focus group discussions are well founded. To manage REACH it is important for Rolls-Royce to identify where SVHCs are used in products, prioritising those that are at a higher risk of being affected for which no alternatives exist. Understanding what the business may have to do to mitigate what is initially a very big risk is then required. It can then be identified where mitigations also carry high risks to seek further alternative actions, such as developing alternatives instead of undertaking engineering design changes.

Managing risks posed to new designs requires prompts to ensure that designs are screened for the use of SVHCs. Along with prompts, a tool to identify where SVHCs are being used in products is also required to manage the risks posed by REACH, which is also being developed by the business and which is not too dissimilar from the DfE tool described in chapter 1 . How this can be built upon, including requirements for tools to provide life cycle environmental data within the framework, is discussed further in chapter 7.

From the assessment presented, it is unlikely that the energy hazard will pose a significant threat to product cost objectives in the near future. However, the figure of $£ 87 /$ tonneCO $\mathrm{C}_{2}$ at which energy prices may start to significantly impact on product costs is not orders of magnitude larger than the estimated costs of $\mathrm{CO}_{2}$ from the scenarios of carbon prices reviewed up to 2030 . It would be prudent not to overlook potential risks posed by the energy hazard on the basis of one case study and further assessments are required to verify that the risks posed are insignificant.

Conducting further assessments of the risks posed by the energy hazard would also allow a more accurate assessment of the carbon price at which there is likely to be a significant impact on product cost objectives. Future changes in carbon prices could then be monitored to continually evaluate the probability that this price will ever be reached. If the chances of carbon reaching this price increased significantly actions could be taken to identify where design objectives would be significantly impacted within the business. Mitigating actions could then be taken if necessary, for example developing alternatives to energy intensive manufacturing processes or highlighting to designers where lower energy use manufacturing routes are possible. 
Concerns of energy managers provide a slightly different angle on the energy hazard, focusing on reducing energy use from an operations perspective. Strategic operational energy and carbon reduction targets are set every three years, as briefly discussed in chapter 1 . Investigating risks posed by the energy hazard has highlighted that there is no link between engineering design decisions and how choices of manufacturing route are coupled with operational energy use. An alternative risk approach would be to understand how engineering decisions to choose, for example, a design that requires significant amounts of machining energy, may impact on operational objectives to reduce energy use in the future. If this energy inefficient design allows for products to be more efficient in use, there may be an argument for the business actually using more energy during manufacture. This is an interesting point, although there is little understanding of the relationship between choices of manufacturing route and operational energy use at this time.

\subsection{Summary and conclusions}

This chapter has presented case study scenarios testing the framework for environmental risk management using the REACH and energy hazards identified in chapter 4 . Following the action research cycle defined in the previous chapter, section 6.1 provided more detail on the $\mathrm{REACH}$ and energy hazards to determine the requirements for defining the embedded case studies, which were identifying the use of Substances of Very High Concern (SVHCs) for REACH and quantifying the amount of energy used for a part.

Three case studies were defined. The first assessed risks posed by the use of zinc chromate on the oil tank of the Severn engine to assess risks posed by REACH. The second case study took data collected from a historical DfE study to assess risks posed by the use of trichloroethylene to manufacture a blisk on the Mersey engine. Estimates of the amount of energy used to manufacture the blisk were used to assess risks posed by the energy hazard in the final case study.

Conclusions from a discussion of the results and findings provided in section 6.3 include:

- The risks posed by REACH are significant and it is important that these continue to be managed by the business.

- In particular, it is important for the business to understand the residual risks associated with actions necessary to ensure the continued supply of products once substances become subject to authorisation under REACH. 
- Activities to mitigate the risks from including SVHCs in new designs are also required. Highlighting the risks posed by $\mathrm{REACH}$ will allow them to be balanced with other design requirements. A new design standard is being developed to screen new designs for the use of SVHCs. The method for prioritising substances is similar to the probability assessment method developed for this research.

- Tools to enable the identification of at risk substances are also being developed. These activities represent what could be the first steps towards implementing the framework into the business. This is discussed further in the next chapter.

- From the analysis presented here, it is unlikely that energy prices will increase to a point where there is a significant impact on product costs in the foreseeable future. Similar analyses on other components are required to support this conclusion, which could also lead to the setting of a carbon 'trigger' price at which there may be significant impacts on engineering cost objectives.

- Considering how choices of manufacturing route may impact on the meeting of operational energy reduction targets is an alternative approach for considering the risks posed by the energy hazard, although there is currently no understanding of how engineering manufacturing decisions are coupled with operational energy use. 


\section{Implementation of the framework}

This chapter discusses how the framework could be implemented into Rolls-Royce, and other businesses, fulfilling the final research objective defined in chapter 1 . To begin this discussion, Figure 7.1 summarises how the different parts of the framework have been tested through the research activities presented in chapters 4,5 and 6 .

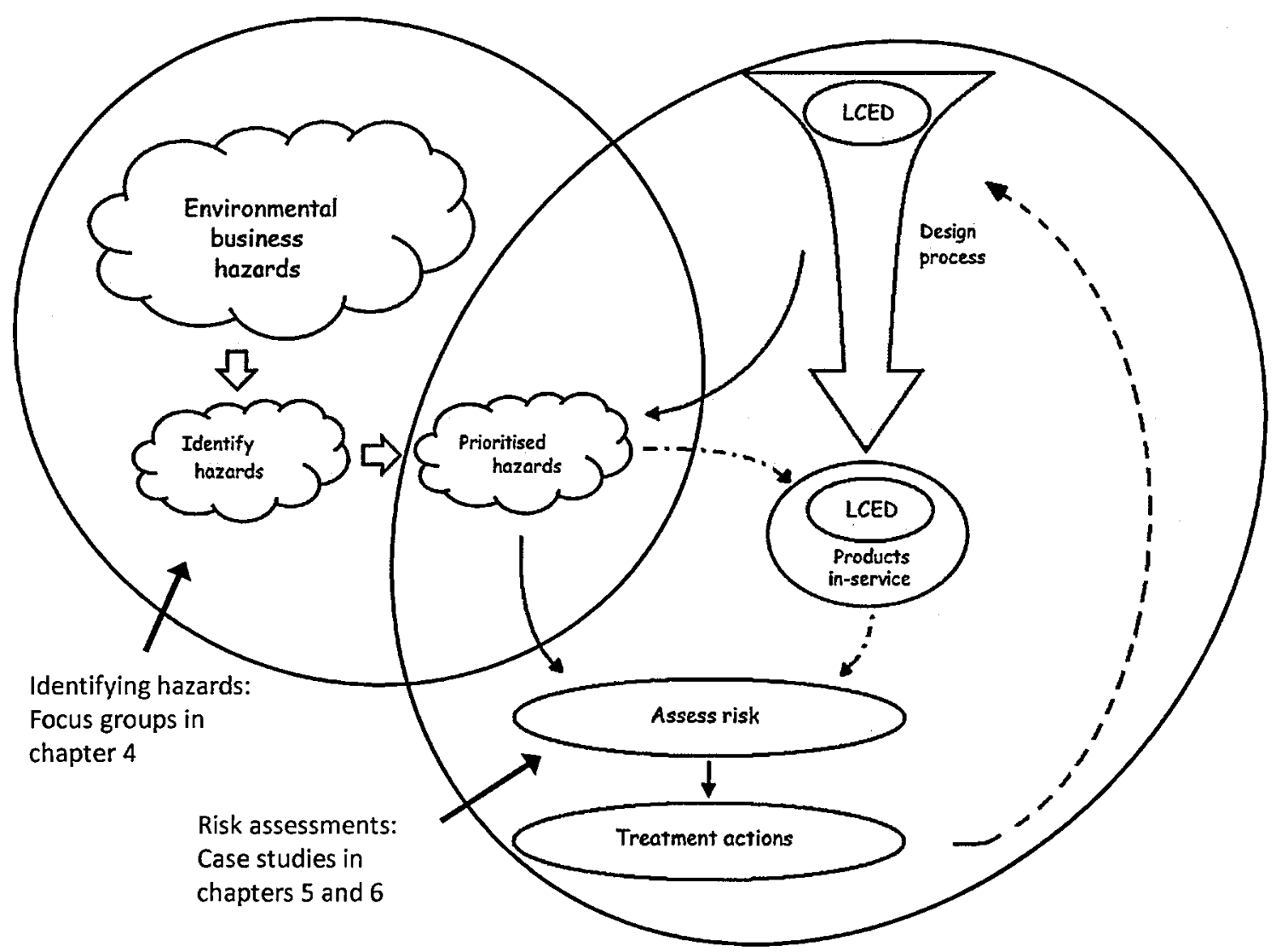

Figure 7.1: Summary of research completed to test the framework

Research to test the framework began in chapter 4, using focus groups to capture the knowledge of Rolls-Royce employees to identify environmental business hazards that were shown to be of concern to the business. These research activities corresponded to the hollow arrows within the framework, leading from environmental business hazards, through identify hazards and on to prioritised hazards. Identifying REACH, access to resources and energy use as environmental business hazards, chapters 5 and 6 presented pilot case studies assessing the risks posed to design and business objectives from these hazards.

The pilot cases showed how the risks posed to new designs could be assessed, corresponding to the solid arrows within the framework leading from the design process into prioritised environmental business hazards and onto risk assessment. Risks posed to products in-service were also assessed, corresponding to the dashed arrows in the framework leading from prioritised hazards to in-service products and onto risk 
assessment. The case studies also discussed how results of the risk assessments could lead into mitigating actions, within design or other parts of the business.

Research activities to test the framework have demonstrated that consideration for environmental risks would be a valuable addition to design decision-making within Rolls-Royce. The pilot cases have shown how the risk based approach fulfils the requirement stated at the beginning of this research, which was to integrate environmental considerations into design decisions by considering their relationship with standard design requirements. It has also been shown how the risk based approach overcomes some of the barriers to integrating environmental considerations into design decisions discussed in chapter 2 , in particular by highlighting the importance of considering non-use phase environmental impacts in design.

The framework for environmental risk management has sufficient merit to be taken forward and implemented in Rolls-Royce. Bearing in mind the stated ambition to produce research outcomes that could lead to change within Rolls-Royce, the following sections provide a discussion on how the framework could be implemented, structured as follows:

1. How the framework could be implemented using the environmental business hazards identified through this research;

2. How the framework could be expanded to manage risks posed by other environmental business hazards beyond those identified through this research;

3. How the framework could be applied in other businesses.

\subsection{Implementing the framework using identified hazards}

This section discusses what is required to implement the framework into Rolls-Royce, focusing on the access to resources and REACH hazards that have been shown to pose a significant threat to the business. How more hazards could be identified and fed into the framework is discussed in section 7.2. Figure 7.2 provides a summary of the requirements that need to be considered for implementing the framework using identified hazards, based on the findings from the case studies. More detail on each of these requirements is given in the following sub-sections, including non-technical factors that might need to be considered to implement the framework, similar to the 'softer' aspects of DfE discussed in chapter 2. 


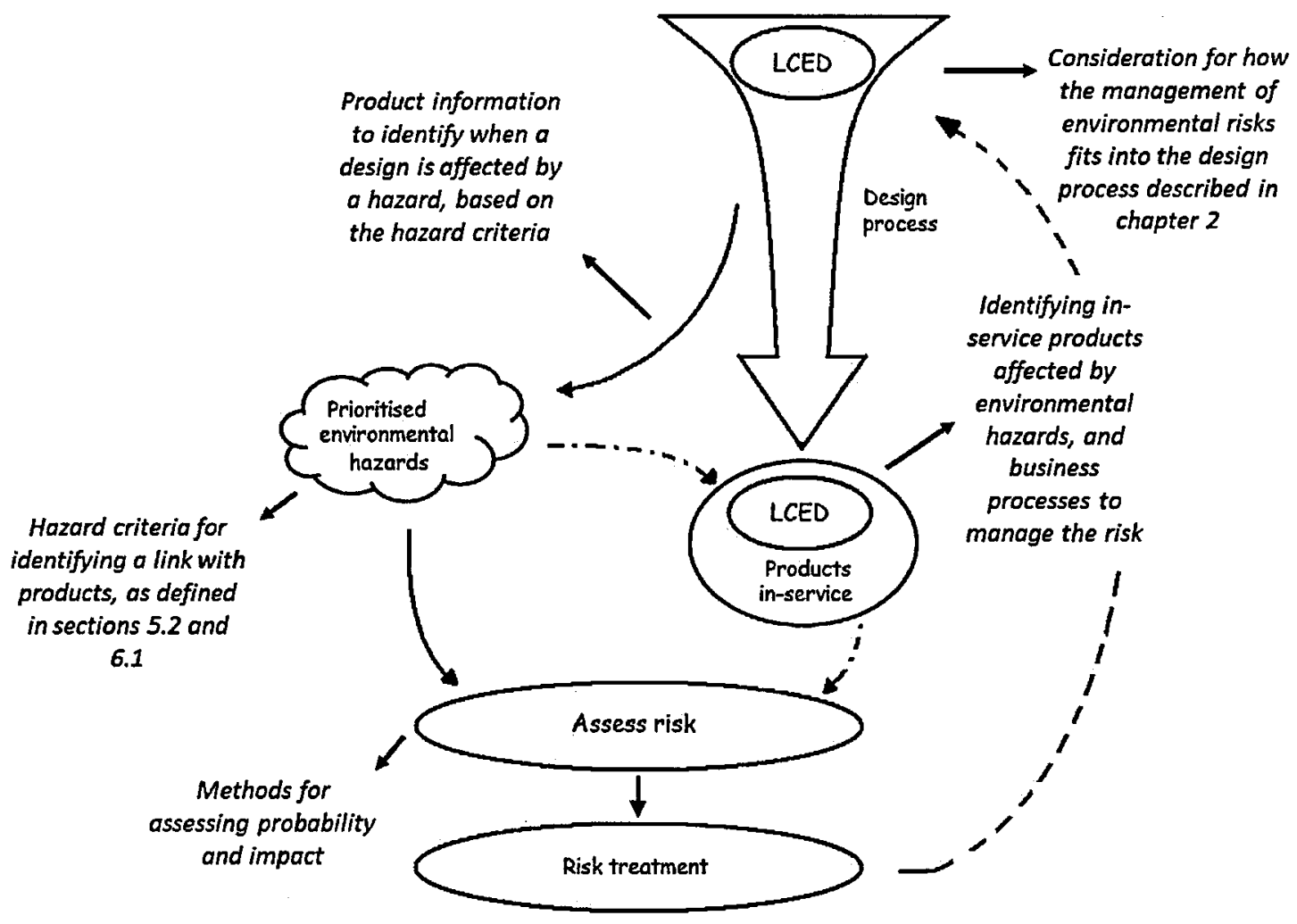

Figure 7.2: Requirements for implementing the framework

\subsubsection{Hazard criteria}

One of the first steps to implement the framework would be to agree on the criteria used to link the access to resources and REACH hazards with products. The first step in completing the case studies presented in chapters 5 and 6 was to provide more detail on the hazards so criteria could be applied to identify a link to a product, which would then allow for an assessment of the risk posed to design and business objectives. For the access to resources hazard the criteria was based on 'at risk' materials identified by the EC report (European Commission, 2010) and for the REACH hazard SVHCs defined by the legal text of the regulation.

Whilst these approaches provided an adequate demonstration of how the framework could work for a pilot study, it was concluded in chapters 5 and 6 that criteria should be developed and agreed within the business that are more reflective of the business' concerns. This included the need to develop a methodology for identifying critical materials, which would form the criteria for highlighting those materials that need to be identified in products. Chapter 6 also concluded that there is a need to develop a method for prioritising SVHCs to be identified in products and it was discussed how this method would benefit from more qualitative inputs from those with knowledge of the legal process, which still remains unclear. Discussing this problem with engineering 
design managers within Rolls-Royce, one proposed approach was to focus on uses of substances with a sunset date to understand the risks posed and what treatment actions were required, and by when.

Research is being funded by Rolls-Royce to complete its own material criticality assessments following on from the findings of this research. Activities are also underway to develop a method for prioritising SVHCs, similar to the approach developed in chapter 6 . Outputs from these activities will define the criteria for linking the access to resources and REACH hazards with products.

\subsubsection{Design workflow and product data}

To implement the framework and integrate consideration for environmental risks into existing business processes requires:

- Definition of how the management of environmental risks fits into the design workflow described in chapter 2;

- Prompts to ensure environmental risks are managed as part of standard risk management activities;

- Design information so it can be identified when a product is affected by an environmental risk, based on the criteria described in section 7.1.1.

Figure 7.3 provides an overview of how the management of environmental risks fits into the standard design framework. Environmental risks need to be managed between design gates 0 and 3 , where the main design decisions are made. At gate 1 a concept is selected for detailed design work. Component materials are confirmed through design gate 2 , leading into the preliminary design review at gate 3 , prior to which decisions over the manufacturing route will also be made. After gate 3 the design, barring the detail, becomes largely fixed and environmental risks need to be considered before this. If not it will be too late to make any alterations without incurring significant design re-work.

Considering environmental risks between gates 0 and 3 fits in with how risks are typically managed through the design process. Design risk management guidance recommends that risks to a design should be identified no later than concept select at gate 1 and actions to treat the risks should be agreed no later than preliminary design review at gate 3. At stage 1 environmental risks related to design concepts could be identified to aid the concept selection decision. Environmental risks should be identified from the material and manufacturing choices confirmed through gates 2 and 
3. Any mitigating actions required could be planned after gate 3 (preliminary design review).

Prompts to enable the identification of risks for design tasks exist and appropriate prompts will be required to ensure environmental risks are considered alongside other risks as part of standard risk management activities. Another way to ensure environmental risks are considered would be to develop appropriate design standards, such as the standard being developed to highlight substances at risk due to REACH, discussed in chapter 6. Questions in design reviews could be also used to ensure environmental risks are being considered.

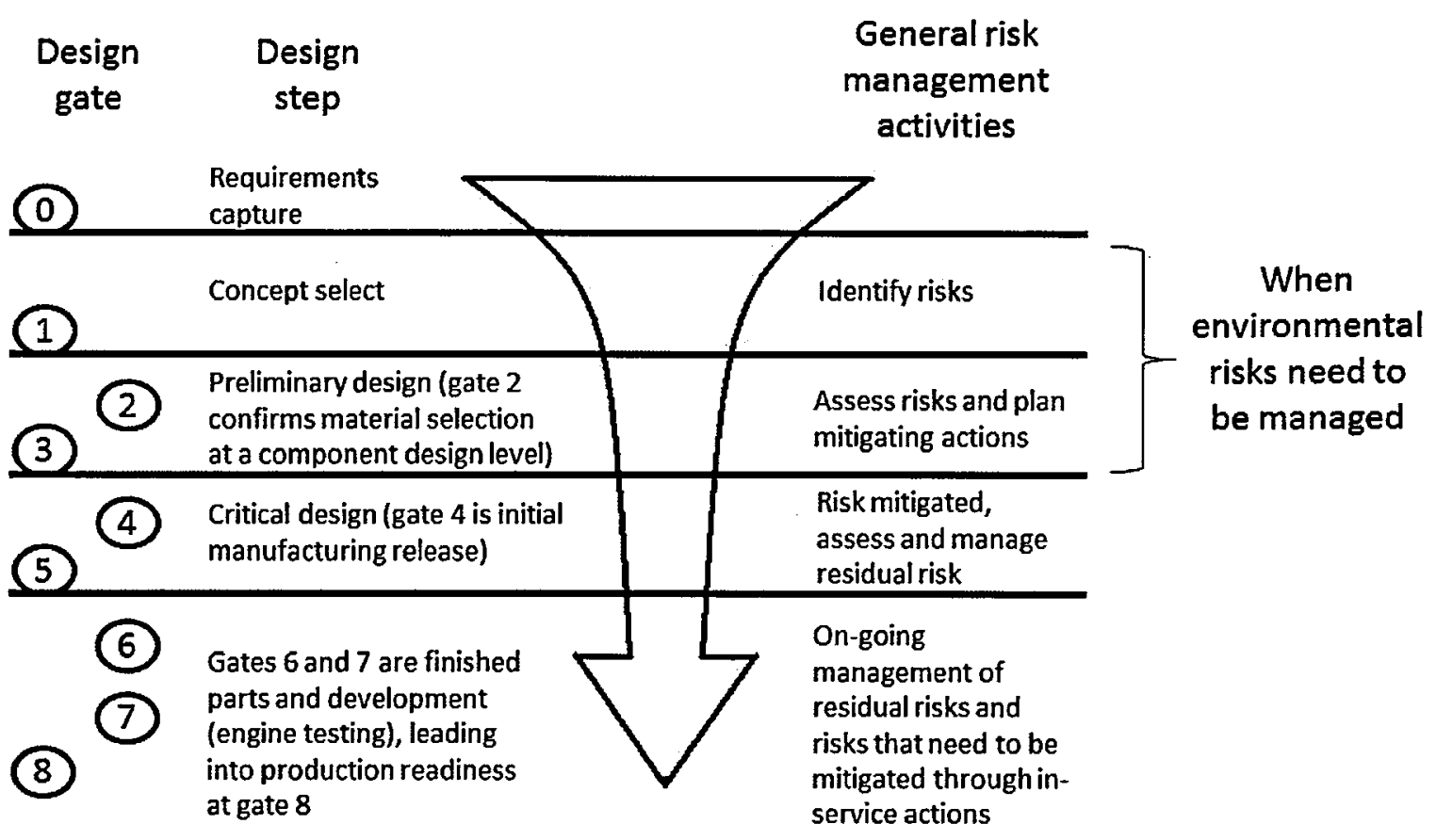

Figure 7.3: Management of environmental risk in the design process

When prompted to do so, designers will require the practical means for identifying when products are affected by the identified hazards, based on the criteria discussed in section 7.1.1. This requires a means of providing information on the substances, materials and processes used to manufacture components. As discussed in chapter 2, approved menus of material and process specifications are used when designing components, which have been proven to be sufficiently technologically mature to be used within, or during the manufacture of, in-service products. One way of ensuring that environmental risks are identified is by flagging those materials and process specifications that contain materials or substances deemed to pose a risk. To do this, it will still be necessary to identify specifications that are related to at risk substances and materials. To manage REACH, product data is already being developed that links manufacturing process specifications to SVHCs, so it can be identified when a part number or new design calls out a specification that uses an at risk substance. 
Managing the access to resources hazard will require a similar data set linking materials specifications to at risk metallic elements, once these at risk elements have been identified.

The nature of the product data required is similar to that produced within simplified life cycle inventories and the need for this type of information is included within the framework as Life Cycle Environmental Data (LCED). A research programme that has been running complementary to this project has begun to develop a design tool to produce this data, building on a methodology for producing life cycle inventories similar to the one described in chapter 1 . The approach links databases of materials and manufacturing processes to designs through Computer Aided Design software, producing a set of product environmental data for that design automatically. Extra effort is not added to the design work flow and hazards within the design can be immediately identified by checking the specifications selected for the design with those that could pose a potential risk to design objectives. Requirements for life cycle environmental data sets are likely to evolve as more hazards are identified, which is discussed further in section 7.2.

\subsubsection{Managing risks to in-service products}

An important part of implementing the framework is ensuring the management of environmental risks posed to in-service products, which was highlighted from the focus group discussions in chapter 4 . From the researcher's experience of working within Rolls-Royce, there are no existing business processes that could be used for managing environmental risks posed to in-service products. If necessary, new or existing business processes will need to be developed and these could be based on the steps to manage risks to in-service products discussed here.

To manage risks to in-service products requires the following:

- Identifying products affected by environmental business hazards, based on the hazard criteria discussed in section 7.1.1;

- An assessment of the risk posed to the business;

- Decisions regarding how the risks are to be treated.

Life cycle environmental data sets for in-service products will be required to identify products affected by environmental business hazards. At risk substances and materials should have been linked to material and process specifications to manage risks posed to new designs, as discussed in the previous section. Extra work will be required to link these specifications to existing part numbers, so at risk substances or 
materials can be quickly linked to affected parts. Activities are already being undertaken within Rolls-Royce to link material and manufacturing specifications to part numbers to assess the risks posed to existing products by REACH. These activities could be easily extended to manage the risks posed to the access to resources hazard as well. This will also be required to report to customers who have to comply with the US Dodd-Frank act, as discussed in chapter 5. A further problem is determining where data on in-service products is stored. The research programme that has developed a means of producing LCED for new designs from CAD software is also investigating how LCED can be stored within the Product Lifecycle Management (PLM) software used within Rolls-Royce.

Once affected parts have been identified, senior managers and engineers on engineering projects would be responsible for understanding the risks posed and what actions are required to treat them, as these are the people responsible for developing and validating designs. Risk management plans, which run throughout the whole PILM cycle (as discussed in chapter 2), will provide the criteria with which to manage risks. The impact of the risk posed to an in-service product is likely to be a lot larger than that posed to new designs, as demonstrated in the case studies, as the continued supply of products and components will be required to fulfil existing business contracts. From discussions with risk managers, it is likely that activities would focus on priority products that either the business wishes to continue selling, or has to continue to supply for maintenance support. With a focus on priority products, it is then likely that judgements will be made on the difficulty of the engineering changes required to treat the risks posed, allowing an assessment of the magnitude of the problems and what might be the best solutions. Support for older products may simply be discontinued if actions to treat risks are too costly.

\subsubsection{Assessing probability and impact}

Whether a new design or in-service product has been identified as being affected by a hazard, methods for assessing probability and impact are required to understand the risk. Focusing on assessing probability first, in the case studies a probability assessment method was developed based on the criteria for linking hazards to products (described in section 7.1.1), which were extrapolated to be used within a standard PID. For example the scores of supply risk for the access to resources hazard were translated into a scale consistent with a probability axis of a PID, as were the steps of the REACH regulatory process for substances requiring authorisation. 
However, as with the need to develop new hazard criteria (discussed in section 7.1.1), there is also the need to develop a new probability assessment method that is more reflective of the business' concerns. This will certainly be required if new hazard criteria are developed, as this will render the probability assessment method used for this research obsolete (as it will be based on the old hazard criteria). A new probability assessment method could be developed by extrapolating hazard criteria onto a probability assessment scale from a risk PID (as was done here), or a different approach could be taken. For example, a method for assessing the probability of risks posed by REACH could be developed based more on expert judgement than the legal text of the regulation, as concluded in chapter 6 . Developing a probability assessment method for the access to resources hazard would also benefit from qualitative inputs from purchasing managers within Rolls-Royce, who can intuitively highlight areas of concern based on their experiences of working within elemental trading. Whatever approach is adopted it must be agreed by the business and be reflective of business concerns.

Assessing the impact of a risk is inevitably dependent on the design context and risk management criteria defined for a particular project. It would not be possible, or even desirable, to develop a universal way of assessing impact in all situations where a risk assessment is being performed. However, some guidance might be required to assess impact, which will have to be agreed. This might include developing a method for judging how the price of a material might change in the future, whether this is using a simple assessment of the ten year elemental price volatility or some other means. Feedback suggested that presenting such a large impact for the REACH hazard was not particularly useful, and also slightly alarmist (as described by one manager), as the business is going to undertake whatever actions are necessary to continue making products. As concluded in chapter 6, a more practical approach would be to assess the difficulty of actions required to treat risks, prioritising actions for substance applications where the residual risks of mitigating the original risk posed by $\mathrm{REACH}$ are also high. For example, where significant design changes are required or where no suitable alternative substances currently exist. Some engine projects in Rolls-Royce's Defence Aerospace business unit have already undertaken a similar activity for some substances (Haneef, 2011).

\subsubsection{Non-technical factors}

To achieve change and implement the framework will require non-technical factors to be considered, similar to the 'softer' considerations for effectively implementing DfE 
discussed in chapter 2. Some of these factors supported the decision to use risk for integrating environmental considerations into design decisions, leading to the development of the framework for environmental risk management. In particular the need to develop an approach tailored to the requirements of a particular context based on an understanding of the product development systems in place.

Now that the framework has been developed and shown to work, considerations for other non-technical factors are required in order to implement it, including:

- The need for senior management commitment, which was highlighted as being particularly important by Delay-Saunders (2006);

- Organisational culture and how this may affect how environmental risks are perceived.

To date the development of the framework for environmental risk management has been supported by an individual business within Rolls-Royce from a relatively low management level, largely in response to the perceived inadequacies of approaches to DfE developed previously (as discussed in chapter 1 ). To implement the capability will require the agreement and support from more senior managers who have responsibility for engineering methods used across Rolls-Royce. Some responsibility for the management of environmental issues also resides with the corporate environmental function, which will also have to buy-in to and promote the implementation of the framework in order for it to be successful. The results of this project have successfully piloted the framework and shown the benefits of considering environmental risks in design, which is an important step towards gaining management commitment for implementing it.

A strong hierarchical culture is evident within Rolls-Royce, influenced by the history of the organisation and the nature of the products it makes. This can mean that changes and new ways of working are developed incrementally and it takes time to implement new ideas and ways of thinking. These conservative attitudes coupled with the fact that environmental risks can be relatively nebulous could lead to differences in how environmental risks are perceived. These differences will have to be resolved in order for the framework to be successfully implemented. For example, some managers within Rolls-Royce have expressed the view that environmental risks are not significant enough to warrant consideration within design decisions, concluding that the business would be able to deal with any significant problems as and when they arise.

The case studies would have benefited from more feedback on how these types of risks are perceived, by completing the risk assessments within actual design activities. From the researcher's experience on how environmental risks might be perceived, 
designers and senior managers within Rolls-Royce typically see the benefits of managing environmental risks and are supportive. Most resistance to change arises with middle managers and one reason for this could be that these are the people who usually control budgets. For this to be overcome clear financial benefits from managing environmental risks need to be demonstrated and the risk assessment results produced from the case studies will help to show this.

\subsection{Expanding the framework to manage new hazards}

Once the framework has been implemented using existing hazards it is interesting to consider how the framework could be applied to other hazards and how the identification of new hazards could be implemented into the business. The following sub-sections discuss:

- How more hazards could be identified;

- How these hazards would need to be managed across the business;

- Practical requirements for managing newly identified hazards.

\subsubsection{Identifying more hazards}

Conclusions from chapter 4 provided recommendations for how more hazards could be identified, including:

- Identifying hazards with a broad scope, covering tactical and strategic problems related to the topic of sustainability.

- Including some form of horizon scanning activity within the process of identifying hazards, considering new hazards that might arise up to 10 years in the future.

- Taking a more focused approach, considering each life cycle stage separately, and involving participants with a greater knowledge of the topics being discussed.

- Developing a different means of prioritising hazards, with decisions being made with the approval of a formally recognised corporate body.

Putting these points together, Figure 7.4 presents a model for identifying environmental business hazards, summarising recommendations on how hazards could be identified in the future within Rolls-Royce. To provide more of a focus it is recommended to run separate hazard identification events, organised per life cycle stage, involving the relevant practitioners and stakeholders within the business who have sufficient 
knowledge of the topics being discussed. More informal workshops could be facilitated internally, consistent with existing risk management approaches.

Information feeding in from these groups should be complemented by a horizon scanning process. This could be conducted by business unit, as each business operates in a different market, which could lead to different environmental drivers and hazards. Although, like REACH and access to resources, some hazards are also likely to be applicable to all businesses. Inputs into the horizon scanning process could include sources of primary and secondary information, for example, views expressed by customers on their environmental requirements and concerns, or information gathered from internet sources and industry magazines or journals. Prompt lists could be developed to guide both the workshops discussions and horizon scanning activity. These could be developed from historical issues and could also draw upon existing guidance, for example that provided by the Sigma Project (see Projectsigma, 2003).

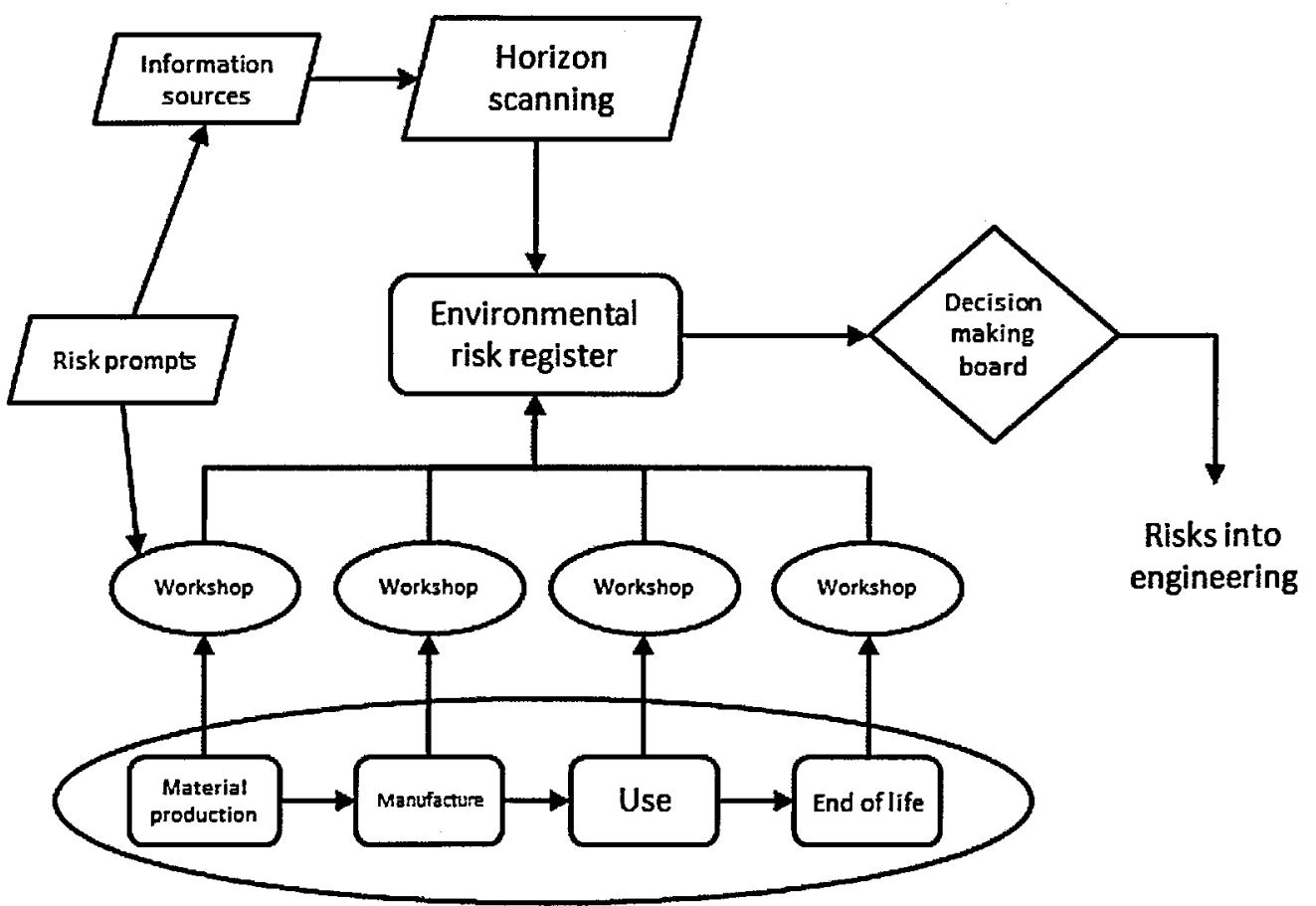

Figure 7.4: Conceptual model for identifying environmental business hazards

The results of the workshops and horizon scanning activity need to be captured and recorded. From discussions with risk managers it is recommended to do this through a corporate environmental risk register. A decision making body is required to decide the priority issues on this register that need to be communicated to engineering to understand the risk through identifying a link between hazards and products. The existing Materials Stewardship Board is an existing corporate decision making body that has the authority to make these judgements. 


\subsubsection{Cross-functional working}

Once prioritised hazards have been agreed these need to be communicated to engineering so new designs and in-service products affected by hazards can be identified to understand and manage the risk. Communicating hazards to engineering will require new links between environment and engineering functions, as shown in Figure 7.5. Managers who are responsible for managing environmental problems typically sit in environmental functions. However, it is designers and engineers who are responsible for creating valid solutions that will actually be affected by environmental risks should they become a reality. Information owned by engineering is also required to identify products affected by hazards to understand the risks identified by environmental functions.

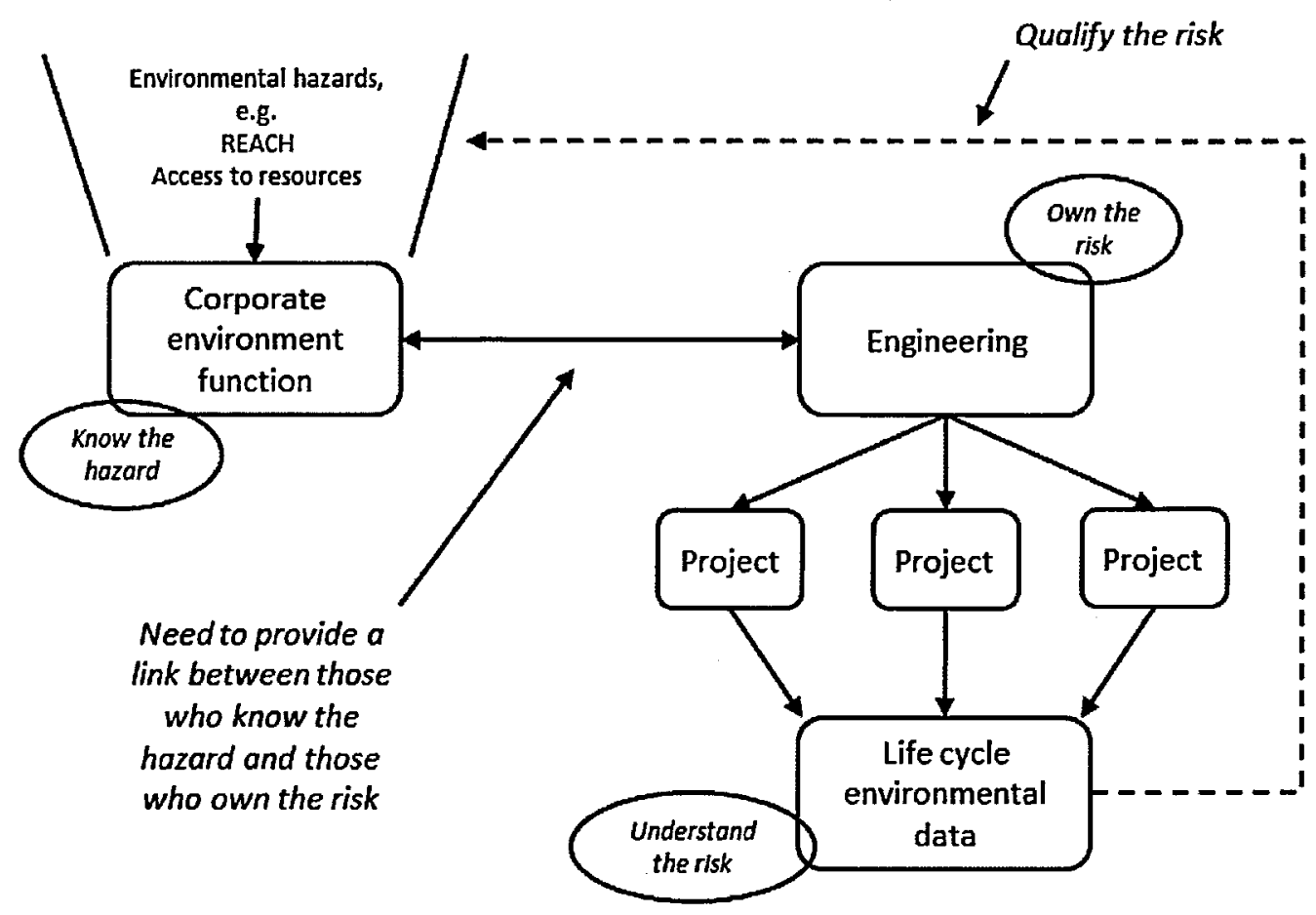

Figure 7.5: Cross-functional working required to implement the framework

Cross-functional working relationships could be supported by creating a network of engineers who have knowledge of environmental risks and how they can be managed. Existing networks of engineers who have specialist knowledge in risk management could be used, adding environmental risks as a further consideration. 


\subsubsection{Practical requirements}

Figure 7.6 outlines steps within a hazard introduction process, built on the practical requirements for implementing the framework discussed previously, that could be applied to manage new hazards within the business.

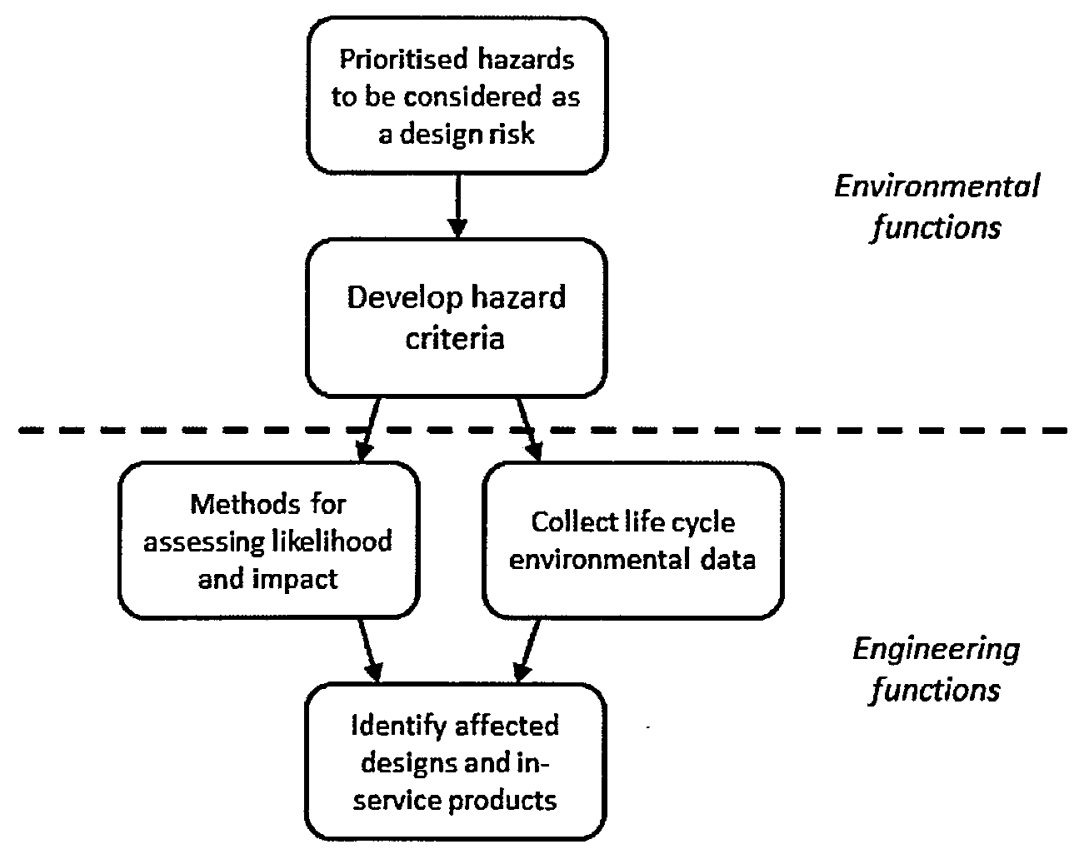

Figure 7.6: Hazard introduction process

Once a hazard has been identified and prioritised for consideration as a design risk, it will be necessary to develop hazard criteria to identify affected products and methods for assessing the probability and impact of the hazard within risk assessments. Life cycle environmental data relevant to the hazard will also have to be collected and incorporated into existing databases, so it can be identified when a design or in-service product is affected by that hazard. Additional prompts will also be required to ensure that new hazards are considered alongside others in risk management activities.

The separation of environmental and engineering functions in Figure 7.6 reflects the current lack of cross-functional working within the business for the management of environmental risks. Over time more integrated ways of working should develop, ensuring that engineering functions understand the need to manage environmental risks and environmental functions develop solutions for managing risks that are appropriate for use in design. 


\subsubsection{Summary}

There will be more hazards that need to be managed by the business in the future. Anecdotal evidence from discussing the topic of environmental risks within RollsRoyce, and with environmental professionals from other businesses, suggests future areas of concern could include:

- Identifying and managing environmental risks from the manufacturing phases of the life cycle, particularly as this research started after significant impacts on design objectives were identified from the use of chemical machining processes;

- Future producer responsibility legislation affecting the aerospace sector;

- Sustainable procurement requirements, in particular from Defence customers.

The only way to show how new environmental business hazards could be managed through the framework is to identify them and investigate whether or not the framework works in these new circumstances. Based on the findings of this research it cannot be claimed that the framework, and ideas developed with it, will be universally applicable to all environmental business hazards. However, the framework has been shown to work for the hazards identified and tested through this research. It would be interesting to apply the framework to some new problems.

A further final consideration is how environmental business hazards need to be considered in other parts of the business, beyond tactical engineering design decisions, and how the framework could be applied or adapted to achieve this. Findings from the focus groups suggested identifying hazards with a broad scope, including strategic issues related to the broader topic of sustainability. It is likely that some of these broader issues will need to be considered in other parts of the business, for example strategic decision making. Whilst outside of the scope of this research, it should be investigated how the framework could be applied or adapted to achieve this through further work.

\subsection{Applying the framework within other businesses}

Using risk management within design is a common and important part of standard engineering practice and most engineering companies apply risk management within design processes. Other businesses would gain from considering environmental risks as part of their design risk management activities and the framework could be used to achieve this. Applying the framework is particularly likely to be beneficial to those 
organisations that face similar engineering challenges to Rolls-Royce, especially those that make technologically advanced long-life products, for example other businesses within the aerospace industry.

Whilst the framework may be applicable within other businesses as a set of interrelated concepts, there are likely to be significant differences in how it is actually applied. Other businesses should apply the framework in a way that fits with the particular needs of that organisation. Hazards identified are also likely to be different, requiring different methods for being managed, whilst maintaining the overall principles outlined by the framework.

\subsection{Summary and conclusions}

This chapter has discussed the implementation of the framework. The following conclusions can be taken from discussions:

- This research has shown that the framework for environmental risk management has sufficient merit to be taken forward and implemented in Rolls-Royce.

- To implement the framework using the REACH and access to resources hazards requires the agreement of hazard criteria that can be used to link these hazards to products. Hazard criteria developed through this research were appropriate for testing the framework, however the business should now develop criteria that is more reflective of the business' concerns and agreed by the appropriate decision making board.

- Figure 7.3 provided an overview of how the management of environmental risks should fit in the standard design work flow, showing how it fits in between design review gates 0 and 3 . Questions in design review gates could also be used to ensure environmental risks are appropriately considered.

- Design information is required to identify when a product is affected by a hazard, which is included within the framework as 'life cycle environmental data'. This data should highlight when a design calls out a specification linked to at risk materials or substances. A separate research programme has developed a means of linking specifications and related materials and substances to designs within CAD software, so it can be identified if a design is affected by an environmental business hazard within the normal workflow. 
- There are no existing business processes for managing the risks posed to in-service products from environmental business hazards. Managing these risks requires a means of identifying products affected by hazards, through extending life cycle environmental data sets to in-service products. A separate research programme is developing a means of storing LCED on in-service products within the PLM software used in Rolls-Royce. These data sets are already being developed to manage risks posed by $\mathrm{REACH}$ and similar data will need to be developed to allow the business to comply with Dodd-Frank, which could be extended to manage risks posed by the access to resources hazard. Once the risk is understood, senior engineering managers are responsible for deciding how to treat them. From discussions with risk managers, support for older in-service products may simply be discontinued if actions to treat risks are costly.

- Agreed methods for assessing the probability and impact of environmental business hazards are required, similar to the need to develop agreement on the hazard criteria discussed previously. New methods for assessing probability would benefit from more qualitative inputs from specialists and managers who have knowledge of the environmental business hazards and could provide a viewpoint more reflective of business concerns. For REACH in particular, assessing impact should focus on the actions required to treat the risks, as highlighting the original risk posed (i.e. all the business related to the affected products) was perceived as being less useful.

- Non-technical factors also need to be considered in implementing the framework, including the need for senior management support and how organisational culture may affect the way environmental risks are perceived. Highlighting the financial benefits of implementing the framework should help gain support from middle management.

- Once the framework has been implemented to manage known hazards it should be expanded to manage the risks posed by new hazards. Figure 7.4 provided an overview of how new hazards could be identified, including the need to incorporate a horizon scanning process and separating out the prioritisation of hazards as the responsibility of an appropriate decisionmaking board.

- Other considerations for managing the risks posed by new hazards include the need to develop new cross-functional working relationships as well as practical requirements, including extending life cycle environmental data sets. 
- Findings from the focus group discussions suggested the need to identify hazards with broad scope, which is likely to pick-up hazards that need to be considered in other parts of the business beyond design decision-making. How the framework could be adapted or applied to manage this should be explored through further work.

- As most engineering companies manage risk in design it is likely that the framework could be applied in other business, as a set of inter-related concepts. However, there are likely to be significant differences in how it is actually applied in practice. 


\section{Conclusions}

This chapter begins by providing a summary of the research in section 8.1 . Section 8.2 summarises research findings and recommendations for Rolls-Royce on how to implement the framework. Section 8.3 lists the contributions to knowledge. A critique of findings and research methods is provided in section 8.4. Section 8.5 provides recommendations for further work.

\subsection{Research summary}

The aim of this research was to develop a bespoke decision support framework for integrating environmental considerations into design decisions, that allowed for environmental impacts to be related to standard design requirements and included in design trade-offs. An action research approach was applied, as the research was taking place in-context within Rolls-Royce with the aim of producing outcomes that could lead to change in the organisation. This applied iterative action research cycles to address the stated research objectives:

1. To investigate how environmental considerations could be integrated into design decisions within Rolls-Royce and select an approach for achieving this;

2. To investigate the chosen approach for integrating environmental considerations into design decisions and develop the bespoke decision support framework;

3. To test the framework through appropriate research activities, showing how it could work within Rolls-Royce;

4. To use the findings from testing the framework to provide recommendations on how to implement it within the business.

Chapter 2 began the first action research cycle to investigate how environmental considerations could be integrated into design decisions within Rolls-Royce. A review of academic literature on the topic of DfE concluded that any approach must be developed relevant to the business and design contexts, on the basis of clear environmental priorities and in a manner consistent with existing design processes. Perspectives on the organisational culture and structure within Rolls-Royce supported the need to develop an approach consistent with existing processes and ways of working. From reviewing design processes it was concluded that using risk could be an appropriate way of integrating environmental considerations into design decisions. 
A new action research cycle was started to address the second research objective and investigate how risk could be used to integrate environmental considerations into design decisions, leading to the development of the framework for environmental risk management. From a review of academic literature a perspective on risk was defined, focused on assessing risks within the frame of business risk management, although with a specific emphasis on issues related to the environment. Similar to the model of environmental risk defined by Matten (1995), this highlighted how environmental risks do not come from the environment itself, but from stakeholder responses to environmental impacts (whether actual or perceived). This led to the definition of the following terms for this research:

- Environmental business hazard: stakeholder responses to environmental impacts with the potential to cause harm to business objectives;

- Environmental risk: multiplying the probability of an environmental business hazard by its potential impact on business objectives;

- Environmental risk management: the activities of identifying, assessing and treating environmental risks.

A review of risk management within Rolls-Royce concluded that a means of ensuring environmental risks are considered like any other risk within standard design risk management practices was required. The framework for environmental risk management (Figure 3.15) was built by defining the various elements necessary to achieve this, and their interrelationships, including:

- The need to represent environmental risks and the design process;

- Steps for the identification of environmental business hazards;

- Considerations for how hazards also affect in-service products;

- The need for life cycle environmental data to link hazards and products;

- Standard steps within risk management, including assessing risk and mitigating actions, some of which may take place within the design process.

Chapter 4 began a new iteration of the action research cycle, addressing the third research objective of testing the framework for environmental risk management and specifically addressing the problem of identifying environmental business hazards. Focus groups were selected as the research approach to identify hazards, based on a requirement to capture the knowledge of environmental specialists and business managers within Rolls-Royce and develop consensus through group discussions. $\mathrm{REACH}$, access to resources and $\mathrm{CO}_{2}$ /climate change and energy costs were identified as priority hazards, which were shown to be of concern to Rolls-Royce. Findings from the focus group discussions also provided insights into how the problem of identifying hazards could be tackled by the business in the future. 
Chapters 5 and 6 began a new action research cycle, maintaining the research theme from chapter 4 to test the remainder of the framework using case studies based on the hazards identified. Chapter 5 presented case studies assessing risks posed to design and business objectives by the access to resources hazard. A review of the access to resources hazard identified critical materials thought to be at risk of supply restrictions. Two case studies were presented focusing on the use of ruthenium in the IPT blades of the Thames engine and yttrium in the combustor outer liner of the Severn engine, assessing the risks posed by developing methods to understand the probability and impact of the risk in a format consistent with existing risk management practices. Results showed that risks posed by the access to resources hazard can be significant and how the risk approach allows for environmental considerations to be traded-off with other design requirements, which was one of the main aims of this research. Findings from the case studies also provided recommendations on how the business could manage the risks posed by the access to resources hazard in the future.

Chapter 6 presented case studies assessing risks posed by the REACH and energy hazards. Exploring the hazards, it was identified that to assess the risks posed by $\mathrm{REACH}$ required the identification of SVHCs used in products and to assess the risks posed by the energy hazard required an assessment of the amount of energy to make components. The first case study, assessing risks posed by $\mathrm{REACH}$, focused on the use of zinc chromate on the oil tank of the Severn engine and the second case study focused on assessing risks posed by the use of trichloroethylene in the manufacture of a blisk on the Mersey engine. Both risk assessments were completed by using risk criteria taken from business risk management plans and developing methods for assessing the probability and impacts of the risk using this criteria. Results showed that the risks posed by REACH were significant, reflecting concerns expressed during the focus group discussions in chapter 4 . It was also suggested to focus on the actions required to mitigate risks posed by $\mathrm{REACH}$ to assess the likely impact on the business. For example, whether fundamental design changes are needed or minimal changes to specification due to the use of an alternative substance during manufacturing. Risks posed by the energy hazard were shown to be insignificant and from the analysis presented it was concluded energy prices are unlikely to increase to a point where there will be a significant impact on product costs in the foreseeable future. Similar analyses on other components are required to support this conclusion.

Chapter 7 addressed the final research objective, discussing what was required to implement the framework into the business. Brief discussions also focused on whether the framework could be applied or adapted to manage environmental risks as part of 
other business decisions within Rolls-Royce and whether the framework could be applied in other businesses.

\subsection{Summary of findings and recommendations}

Summarising findings and recommendations with reference to the research objectives:

1. To investigate how environmental considerations could be integrated into design decisions within Rolls-Royce and select an approach for achieving this.

Risk was selected as the approach for integrating environmental considerations into design decisions. A risk based approach would:

- Allow environmental impacts to be considered in a way that was consistent with existing processes and ways of working;

- Present environmental impacts in a format that could be traded with other design requirements;

- Highlight the importance of addressing non-use phase environmental impacts;

- Point designers to actions that can be taken to reduce environmental impacts.

2. To investigate the chosen approach for integrating environmental considerations into design decisions and develop the bespoke decision support framework.

Investigating how risk could be used to integrate environmental considerations into design decisions led to the definition of a perspective on risk that was set within the context of business risk management although with a specific focus on environmental impacts. The framework for environmental risk management was defined for considering environmental risks as part of standard design risk management practices (Figure 3.15).

3. To test the framework through appropriate research activities, showing how it could work within Rolls-Royce.

Activities to test the framework began with identifying environmental business hazards through the use of focus groups conducted within Rolls-Royce. Prioritised hazards shown to be of concern to Rolls-Royce included:

- Access to resources;

- REACH;

- Climate change/ $\mathrm{CO}_{2}$ and energy costs. 
Findings from the first set of case studies assessing risks posed by the access to resources hazard included:

- Risks posed to design objectives from the access to resources hazard were shown to be significant. From the results it was also concluded that managing risks posed by the access to resources hazard should focus on where at risk materials are used in applications where the costs of these materials forms a larger fraction of the overall unit cost of parts. For example, where the material is used within the material alloy of a part rather than within a coating.

- It was shown how the risk approach allowed for the access to resources hazard to be considered in a way that could be traded with other design requirements.

- A bespoke approach for assessing the probability of material supply restrictions is required and further research is being funded within RollsRoyce to develop this. A framework for criticality assessments (Figure 5.14) was developed, which outlines the steps for completing an assessment. Applying this will allow critical materials relevant to Rolls-Royce to be identified.

- Using historical price volatility was proposed as an acceptable approach for assessing the impact of a material supply restriction, given the complexity of the task of predicting future material prices.

- Related to the access to resources hazard, requirements for businesses that use 'conflict minerals' to report to the US Securities and Exchange Commission under the US Dodd-Frank act highlight potential reputational risks and the importance of continuing to manage these issues in future. This also highlights the need for product life cycle environmental data to track where affected materials are used in products so declarations can be made to customers when required.

Findings from the second set of case studies focusing on the $\mathrm{REACH}$ and energy hazards included:

- The first two case studies focusing on the REACH hazard showed that the risks posed to design and business objectives were significant.

- A better understanding of the potential impacts of the risks posed by $\mathrm{REACH}$ would be gained by understanding the scale of actions required to replace the use of substances at risk of phase out.

- Energy prices are unlikely to increase to have a significant impact on product cost objectives in the foreseeable future, although this finding needs 
to be supported through further case studies. The results of further studies could lead to the setting of a 'trigger price' at which there will be a significant impact on costs. Whether energy prices will reach this level could then be monitored on an on-going basis.

- Another way of viewing the energy hazard would be to analyse how engineering decisions influence energy use in business operations and whether or not choices of manufacturing technologies may impact on the business' ability to meet energy reduction targets.

The framework was claimed as valid, having tested it and successfully shown how it could be used to assess the environmental risks posed to designs and in-service products within Rolls-Royce.

4. To use the findings from testing the framework to provide recommendations on how to implement it within the business.

Discussing how the framework could be implemented led to the following recommendations for achieving this using the hazards identified through this research:

- Developing agreed hazard criteria to form the basis of links between hazards and products. These criteria need to be reflective of the business' concerns and agreed by an appropriate decision making board.

- Ensuring the management of environmental risks is integrated into the standard design work flow, being considered within normal risk management activities between design gates 0 and 3 as shown in Figure 7.3.

- Implementing a capability for supplying the required product data in design, linking materials and process specification picked by designers with at risk substances and materials (identified through hazard criteria), labelled within the framework as 'life cycle environmental data'. Research activities linked to this project have developed a capability demonstrator showing how this data could be provided through CAD software.

- Developing new business processes to manage the risks posed to inservice products, including the development of life cycle data sets, which could be stored in PLM software.

- Agreeing methods for assessing the probability and impact of environmental risks, consistent with the scoring approaches used within standard risk assessment matrices.

- Considering the non-technical factors required to successfully implement the framework, including the need for senior management commitment. 
It was also discussed how the framework could be extended to identify and manage new hazards, leading to the following recommendations:

- Identifying new environmental business hazards using the conceptual model shown in Figure 7.4, which was built on findings from the focus groups presented in chapter 4 ;

- Developing new cross-functional working relationships connecting environmental managers who have knowledge of environmental risks with engineering managers who own them. A model showing how these new working relationships interact was shown in Figure 7.5;

- Considering the practical requirements for managing the risks posed by new environmental business hazards, included within the hazard introduction model shown in Figure 7.6.

\subsection{Contributions to knowledge}

Two main contributions to knowledge are claimed from this research:

1. The development of a risk based approach to $D f E$, the framework for environmental risk management for implementing this type of approach (published in Lloyd et al., 2012a) and testing the framework through pilot case studies within an organisation.

This research has presented the first approach for integrating environmental considerations into design decisions that is explicitly based on risk, based on evidence from the literature reviewed. The framework provides a visual representation of the different elements required to manage environmental risks in design, and the interrelationships between these elements, allowing for this concept to be applied within Rolls-Royce and other businesses. Showing how the framework could work through case studies was crucial to developing recommendations on how it could be implemented into a business.

2. The second contribution is the framework for conducting material criticality assessments, which was developed from merging findings from a review of materials criticality studies with pre-defined attributes of effective decision support in complex contexts (published in Lloyd et al., 2012c).

First defined by National Research Council (2008), materials criticality seeks to understand the risks posed by potential restrictions in the accessibility of materials, by assessing the probability of a material supply restriction (often termed 'supply risk') and the potential impact if a restriction were to occur. Whilst there are many materials 
criticality studies available (a comprehensive overview is provided by Erdmann and Graedel, 2011), to the researcher's knowledge there has been no description of the general steps required to complete a criticality assessment. The criticality framework is useful for organisations that may wish to conduct their own assessments, instead of relying on studies produced from other sources.

\subsection{Reflections on research findings and methods}

This section briefly reflects on the research findings and methods applied, including:

- General limitations related to the development and application of a framework, including how the framework for environmental risk management differs from other risk management frameworks;

- The pilot nature of the case studies and how this affects the validity of recommendations on how to implement the framework;

- The suitability of the research methods applied and whether alternative methods could have been used.

Referring to their use during case study research, Robson (1993) defines a 'framework' as covering the 'main features (aspects, dimensions, factors, variables) of a case study and their presumed relationships' ( $p .150)$. Whilst Robson is referring to the use of frameworks within the particular context of case study research, this definition generally holds true for any application of the term, as a description for a visual representation of a system, or set of processes, and their interactions. Most approaches to risk management typically apply a framework of one sort or another, which generally speaking includes the same steps to manage risks, even though the terminology used and perspective on risk may differ (as discussed in chapter 3 ).

Given that frameworks are widely applied in risk management, this does raise the question of how the framework developed for this research is different from those applied elsewhere. The framework for environmental risk management is distinct due to the perspective on risk upon which it is founded, as well as its specific focus on managing environmental risks within the context of engineering design, including the explicit need for product life cycle environmental data. However, given the stated definition of a framework, it cannot be claimed that the framework for environmental risk management presented here is the only way of visually representing the various elements required to manage environmental risks in design. There are likely to be many interpretations, and the framework presented here has developed iteratively over the course of the research and could change again in the future. Any interpretation is 
valid so long as it captures the main elements required to manage environmental risks and their inter-relationships and produces the same results. Developing alternative interpretations might be particularly useful for communicating the framework for use in other businesses, if a way of presenting environmental risk management could be found that is more relevant to an alternative context.

A potential criticism of this research is the pilot nature of the case study scenarios presented in chapters 5 and 6 . An ideal approach would have used case studies based on real-time assessments of products being designed, embedded within the design teams so feedback on the risks assessed could be provided within the design environment. Case study scenarios were used for the following reasons (in no particular order):

- To complete the case studies products affected by the identified hazards had to be selected, which restricted the design tasks that could be used as a case;

- At the time the cases were selected there were limited new design projects available for real time case studies within the defence aerospace business that hosted the research;

- It would not have been practical to gather the required life cycle environmental data to assess if a design was affected by a hazard within the time constraints of a real time project, without the availability of a suitable tool that does this automatically;

- The framework had been developed as a concept at the onset of the case studies which meant they were completed in an exploratory way, learning how the system could work as the case studies progressed. 'Learning through doing' within a real-time design environment would not have been practical. It would have also been difficult to sell the concept of doing the work to a design team without knowing exactly what the outcomes might be;

- The researcher was conscious that the business struggles generally with risk management in engineering and adding new issues to a developing practice might have been problematic.

Whilst the pilot studies were not the ideal approach, using pilot studies did allow for the ideas presented in this thesis to be developed and tested to a point where the framework is ready to be implemented into a real-time business environment. Given the framework was only conceptual before the pilot case studies were completed, it would not have been possible to implement the framework before testing it on a pilotbasis in some way. Hence, the activities presented in this thesis to trial the framework have made an important contribution in moving towards implementation. 
Action research was selected as the most appropriate approach for this research, given the aim of producing outcomes that could lead to change within Rolls-Royce. With hindsight, action research was an appropriate research approach, especially given it promotes greater involvement between the researcher and the researched (Robson, 1993). Following the discussion on the benefits that conducting real time design scenarios would have provided, if anything this research would have benefited from more involvement between business colleagues and the researcher.

Reflecting on the research methods used, combining action research with the use of case studies does align the approach taken closely with the 'action case' described by Braa and Vidgen (1999). The approach taken also fits well with Robson's simplified action research model (Figure 8.1), in that the initial research developed the framework, which was tested (which could align with validation) and interpreted leading to recommendations on how it could be implemented. Hopefully this will lead to action within Rolls-Royce to put the ideas presented here into practice.

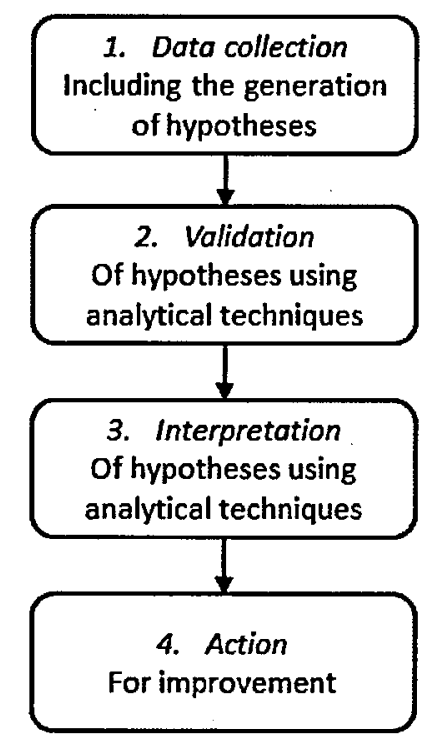

Figure 8.1: Simplified action research model (adapted from Robson, 1993)

Checkland and Holwell (1998) assert that for the findings of action research to be valid, a framework of ideas, methodology and area of concern have to be declared, in particular as these elements are likely to change due to the nature of real-world problem situations. If this declaration is not made the findings of action research cannot be claimed as being any more than anecdotal. The ideas developed for this research, methodologies applied and area of concern have been appropriately described throughout this research, hence on the basis of this test, the research findings presented here are claimed as valid application of action research. 
However, whilst the over-arching research approach was sound, how the different elements of the research problem were approached could have been different. Earlier research that led to the development of the framework could have benefited from a more structured approach, in particular the informal interviews. However, given how little was known on the topic of DfE within the business at the time this might have been difficult. Focus groups successfully provided hazards that could be used to test the framework, however different approaches could have been used. For example, interviews could have been used to get a feel for the important issues. A multi-criteria approach could then have been used to prioritise the important issues that could be used to test the remainder of the framework. All research approaches were selected with a clear view of the desired outcomes, and these were all successfully achieved.

\subsection{Recommendations for further work}

The main recommendation for further work is to follow up the findings and recommendations of this research and implement the framework for environmental risk management into Rolls-Royce. It is recommended to first focus on implementing the framework using the hazards identified through this research. Once systems are in place to manage acknowledged environmental risks, more hazards can be identified and built in to pre-existing processes. Gaining senior management support is a challenge that still needs to be addressed. Showing how the framework could work through this research should help promote the case for implementing it.

Other areas of further work that would be interesting to follow up include:

- Conducting further studies to investigate whether the energy hazard does pose an insignificant threat to design product cost objectives in the foreseeable future. This could also lead to a more accurate carbon 'trigger price' at which the cost of energy intensive manufacturing processes may become unacceptable. It would also be interesting to investigate whether engineering decisions are being made to use more energy intensive processes and whether this may impact on environmental objectives to reduce energy use in operations.

- Investigating how the framework could be applied or adapted to consider environmental risks within other business decisions. In particular more strategic decisions related to the products that Rolls-Royce makes.

- Testing the validity of the framework by investigating how it could be applied in other businesses. 


\section{References}

Adams, J. (1995) Risk. London: UCL Press.

Alonso, E., Gregory, J., Field, F. and Kirchain, R. (2007) 'Material availability and the supply chain: risks, effects, and responses', Environmental Science and Technology, 41 (19), pp.6649-6656.

Ammenberg, J. and Sundin, E. (2005) 'Products in environmental management systems: Drivers, barriers and experiences', Journal of Cleaner Production, 13 (4), pp.405-415.

Apple (2009) Apple and the environment. Available at:

http://www.apple.com/environment/design/ (accessed 18-02-09).

Aven, T. and Renn, O. (2009) 'On risk defined as an event where the outcome is uncertain', Journal of Risk Research, 12 (1), pp.1-11.

Aven, T. and Renn, O. (2010) 'Response to Professor Eugene Rosa's viewpoint to our paper', Journal of Risk Research, 13 (3), pp.255-259.

Bakker, C. (1995) Environmental information for designers. PhD Thesis, TU Delft, Delft, The Netherlands.

Barbour, R.S. and Kitzinger, J. (eds.) (1999) Developing focus group research: Politics, theory and practice. London: Sage.

Bare, J.C., Hofstetter, P., Pennington, D.W., and Udo de Haes, H.A. (2000) 'Midpoints versus endpoints: The sacrifices and benefits', International Journal of Life Cycle Assessment, 5 (6), pp.319-326.

Baumann, H., Boons, F. and Bragd, A. (2002) 'Mapping the green product development field: Engineering, policy and business perspectives', Journal of Cleaner Production, 10 (5), pp.409-425.

Baumann, H. and Tillman, A-M (2004) The Hitch Hiker's guide to LCA. Lund: Studentlitteratur.

Beasley, R. and Clifton, A. (2008) 'The impact of environmental issues on Rolls-Royce design systems and solutions', $18^{\text {th }}$ Annual Symposium of the International Council of Systems Engineering, Utrecht, The Netherlands, 15-19 June.

BGS (2011) Risk list 2011. Available at:

http://www.bgs.ac.uk/mineralsuk/statistics/riskList.html (accessed 10-10-2011). British Geological Survey (BGS).

Bhamra, T.A. (2004) 'Ecodesign: The search for new strategies in product development', Proceedings of the IMechE Part B, Journal of Engineering Manufacture, $218(5)$, pp.557-569.

Bishop, P., Hines, A. and Collins, T. (2007) 'The current state of scenario development: An overview of techniques', Foresight, 9 (1), pp.5-25.

Boeing (2006) Movement improvement. Available at: http://www.boeing.com/news/frontiers/archive/2006/august/ (accessed 16-02-09). pp.30-33.

Boks, C. (2006) 'The soft side of ecodesign', Journal of Cleaner Production, 14 (15/16), pp.1346-1356. 
Börjeson, L., Höjer, M., Dreborg, K-H., Ekvall, T. and Finnveden, G. (2006) 'Scenario types and techniques: Towards a user's guide', Futures, 38 (7), pp.723-739.

Braa, K. and Vidgen, R. (1999) 'Interpretation, intervention, and reduction in the organisational laboratory: A framework for in-context information system research', Accounting, Management and Information Technologies, 9 (1), pp.25-47.

Bradfield, R., Wright, G., Burt, G., Cairns, G. and Van Der Heijden, K. (2005) 'The origins and evolution of scenario techniques in long range business planning', Futures, 37 (8), pp.795-812.

Brady, T. and McManus, T. (2003) 'Design for environment at Intel', $3^{\text {rd }}$ International Symposium on Environmentally Conscious Design and Inverse Manufacturing, Tokyo, Japan, 8-11 December.

Brezet, H. and Van Hemel, C. (eds.) (1997) Ecodesign: A promising approach to sustainable production and consumption. Paris: United Nations Environment Programme.

Brydon-Miller, M., Greenwood, D. and Maguire, P. (2003) 'Why action research?', Action Research, 1 (1), pp.9-28.

BSI (2002) ISO/TR 14062:2002: Environmental management - Integrating environmental aspects into product design and development. London: British Standards Institution (BSI).

BSI (2004) BS EN ISO 14001:2004: Environmental management systems requirements with guidance for use. London: British Standards Institution (BSI).

BSI (2006) BS EN ISO 14040:2006: Environmental management - Life cycle assessment - Principles and framework. London: British Standards Institution (BSI).

BSI (2009) BS ISO 31000:2009: Risk management - Principles and guidelines. London: British Standards Institution (BSI).

BSI (2010) BS EN 31010:2010: Risk management - Risk assessment techniques. London: British Standards Institution (BSI).

Charter, M. and Belmane, I. (1999) 'Integrated product policy (IPP) and eco-product development (EPD)', Journal of Sustainable Product Design, 10 (July), pp.17-29.

Checkland, P. (1993) Systems thinking, systems practice. Chichester: John Wiley.

Checkland, P. and Holwell, S. (1998) 'Action research: Its nature and validity', Systemic Practice and Action Research, 11 (1), pp.9-21.

Checkland, P. and Poulter, J. (2006) Learning for action. Chichester: John Wiley.

Christiansen, K. (ed.) (1997) Simplifying LCA: Just a cut? Brussels: SETAC-Europe.

Cohen, D. (2007) 'Earth audit', New Scientist, 194 (2605), pp.34-41.

Cowell, S.J., Fairman, R. and Löfstedt, R.E. (2002) 'Use of risk assessment and life cycle assessment in decision making: a common policy research agenda', Risk Analysis, 22 (5), pp.879-894.

Cronin, A. (2001) 'Focus groups', in: Gilbert, N. (ed.) Researching Social Life. $2^{\text {nd }}$ Edition. London: Sage. pp.164-177. 
Crul, M.R.M, Diehl, J.C. and Ryan, C. (eds.) (2009) Design for sustainability: a step-bystep approach. Paris: United Nations Environment Programme.

Dash, D.P. (1999) 'Current debates in action research', Systemic Practice and Action Research, 12 (5), pp.457-492.

DECC (2009) Quarterly energy prices: June 2009. London: Department for Energy and Climate Change (DECC).

DECC (2010) Energy price statistics. London: Department for Energy and Climate Change (DECC).

Delay-Saunders, I. (2006) Integrating environmental considerations in aircraft design. Unpublished EngD Thesis, University of Surrey, Guildford, UK.

DETR (2000) Guidelines for environmental risk assessment and management. Department for Environment, Transport and the Regions (DETR), London: The Stationary Office.

Donnelly, K., Beckett-Furnell, Z., Traeger, S., Okrasinski, T. and Holman, S. (2006) 'Eco-design implemented through a product-based environmental management system', Journal of Cleaner Production, 14 (15/16), pp.1357-1367.

Dul, J. and Hak, T. (2008) Case study methodology in business research. Oxford: Elsevier.

Dunjó, J., Fthenakis, V., Vílchez, J.A. and Arnaldos, J. (2010) 'Hazard and operability (HAZOP) analysis: a literature review', Journal of Hazardous Materials, 173 (1-3), pp.19-32.

Earl, G. and Clift, R. (1999) 'Stakeholder value analysis: A methodology for integrating stakeholder values into corporate environmental investment decisions', Business Strategy and the Environment, 8 (3), pp.149-162.

ECHA (2011a) About REACH. Available at:

http://guidance.echa.europa.eu/about reach en.htm (accessed 10-09-2011).

European Chemicals Agency (ECHA).

ECHA (2011b) Authorisation under REACH. Available at:

http://echa.europa.eu/reach/authorisation under reach en.asp (accessed 10-092011). European Chemicals Agency (ECHA).

ECHA (2011c) Candidate list. Available at:

http://echa.europa.eu/chem data/authorisation process/candidate list table en.asp

(accessed 11-01-2013). European Chemicals Agency (ECHA).

Elkington, J. and Trisoglio, A. (1996) 'Developing realistic scenarios for the environment: Lessons learnt from Brent Spar', Long Range Planning, 29 (6), pp.762769.

Erdmann, L. and Graedel, T.E. (2011) 'Criticality of non-fuel minerals: A review of major approaches and analyses', Environmental Science and Technology, 45 (18), pp.7620-7630.

Ernst \& Young (2009) The 2009 business risk report: The top 10 risks for global business. London: Ernst \& Young. 
European Commission (2006) 'Commission regulation (EC) no. 1907/2006 of 18 December 2006 concerning the Registration, Evaluation, Authorisation and Restriction of Chemicals (REACH)', Official Journal of the European Union, L396/1-849.

European Commission (2010) Critical raw materials for the EU. Brussels: European Commission.

Fielding, N. and Thomas, H. (2001) 'Qualitative interviewing', in: Gilbert, N. (ed.) Researching social life. $2^{\text {nd }}$ Edition. London: Sage. pp.123-144.

Fiksel, J. (1996) 'Achieving eco-efficiency through Design for Environment', Environmental Quality Management, 5 (4), pp.47-54.

Finnveden, G. (2000) 'On the limitations of life cycle assessment and environmental systems analysis tools in general', International Journal of Life Cycle Assessment, 5 (4), pp.229-238.

Fischhoff, B., Watson, S.R. and Hope, C. (1984) 'Defining risk', Policy Sciences, 17 (2), pp.123-139.

Fischhoff, B. (1995) 'Risk perception and communication unplugged: Twenty years of process', Risk Analysis, 15 (2), pp.137-145.

Fitzgerald, D.P., Herrmann, J.W., Sandborn, P.A., Schmidt, L.C. and Gogoll, T.H. (2005) 'Beyond tools: A design for environment process', International Journal of Performability Engineering, 1 (2), pp.105-120.

Flyvbjerg, B. (2011) 'Case study', in: Denzin, N.K. and Lincoln, Y.S. (eds.) The Sage handbook of qualitative research. $4^{\text {th }}$ Edition. Thousand Oaks, CA: Sage. pp.301-316.

Gerrard, S. and Petts, J. (1998) 'The relationship between risk assessment and risk management', in: Hester, R.E. and Harrison, R.M. (eds.) Risk assessment and risk management. Issues in environmental science and technology 9. Cambridge: Royal Society of Chemistry. pp.1-20.

Gilbert, N. (2001) Researching social life. $2^{\text {nd }}$ Edition. London: Sage.

Glaser, B.G. and Strauss, A.L. (1999) The discovery of grounded theory: strategies of qualitative research. Piscataway, $\mathrm{NJ}$ : Transaction.

Glazier, L. (2009) Engineering risk management how to. Internal report, Rolls-Royce.

Godet, M. and Roubelat, F. (1996) 'Creating the future: The use and misuse of scenarios', Long Range Planning, 29 (2), pp.164-171.

Gordon, R.B., Bertram, M. and Graedel, T.E. (2007) 'On the sustainability of metal supplies: A response to Tilton and Lagos', Resources Policy, 32 (1/2), pp.24-28.

Graedel, T.E. and Allenby, B.R. (1995) Industrial ecology. Englewood Cliffs, NJ: Prentice Hall.

Great Britain (2008) Climate Change Act 2008: Elizabeth II. Chapter 27. London: The Stationery Office.

Handfield, R.B., Melnyk, S.A., Calantone, R.J. and Curkovic, S. (2001) 'Integrating environmental concerns into the design process: The gap between theory and practice', IEEE Transactions on Engineering Management, 48 (2), pp.189-208.

Handy, C. (1999) Understanding organizations. $4^{\text {th }}$ Edition. London: Penguin. 
Haneef, M. (2011) Hexavalent chrome on 'Severn' engines. Internal report, RollsRoyce.

Harper, P. and Weaver, T. (2003) LCA comparison report. Internal report, Rolls-Royce.

Harrison, R. (1972) 'Understanding your organization's character', Harvard Business Review, May/Jun, pp.119-128.

Hauschild, M.Z., Jeswiet, J. and Alting, L. (2004) 'Design for Environment - Do we get the focus right?', CIRP Annals - Manufacturing Technology, 53 (1), pp.1-4.

Healy, G.T., Hopper, P.J., Ward, A.G. and Wilkinson, B. (1994) Reliability engineering manual. Internal publication, Rolls-Royce.

HMRC (2010) Carbon price floor: support and certainty for low-carbon investment. London: Her Majesty's Revenue and Customs (HMRC).

Hoffman, W.F. (1997) 'Recent advances in Design for Environment at Motorola', Journal of Industrial Ecology, 1 (1), pp.131-140.

HP (2009) Design for environment. Available at:

http://www.hp.com/hpinfo/globalcitizenship/environment/productdesign/design.htm/ (accessed 18-02-09).

ICAEW (1999) Internal control: guidance for directors on the combined code. London: Institute of Chartered Accountants in England and Wales (ICAEW).

Jackson, T. (1996) Material concerns. London: Routledge.

Kaufmann, D., Kraay, A. and Mastruzzi, M. (2010) The Worldwide Governance Indicators. Policy Research Paper 5430. Washington, DC: The World Bank.

Kidela (2011) Yttrium: a versatile and sought after rare earth. Available at: http://www.kidela.com/kidela/yttrium-a-versatile-and-sough-after-rare-earth (accessed 12-10-2011).

Kletz, T. (1999) HAZOP and HAZAN. $4^{\text {th }}$ Edition. London: The Institution of Chemical Engineers.

Koizumi, Y., Kobayashi, T., Jianxin, Z., Yokokawa, T., Harada, H., Aoki, Y. and Arai, M. (2003) 'Development of next generation Ni-base single crystal superalloy', Proceedings of the International Gas Turbine Congress, Tokyo, 2-7 November. Tokyo: Gas Turbine Society of Japan.

Krueger, R.A. and Casey, M.A. (2009) Focus groups: A practical guide for applied research. $4^{\text {th }}$ Edition. Thousand Oaks, CA: Sage.

Lagerstedt, J. and Luttropp, C. (2006) 'Guidelines in ecodesign: A case study from the railway industry', in: Brissaud, D. et al. (eds.) Innovation in Life Cycle Engineering and Sustainable Development. New York: Springer. pp.245-254.

Lee, C-H. (1998) 'Formulation of resource depletion index', Resources, Conservation and Recycling, 24 (3/4), pp.285-298.

Lee, J.J. (2006) 'Greener manufacturing, maintenance and disposal - towards the ACARE targets', The Aeronautical Journal, 110 (1110), pp.567-571.

Leitch, M. (2010) 'ISO 31000:2009 - the new international standard on risk management', Risk Analysis, 30 (6), pp.887-892. 
Lempert, R., Hallsworth, M., Hoorens, S. and Ling, T. (2009) Looking back on looking forward: A review of evaluative scenario literature. Copenhagen: European Environment Agency.

Lenox, M. and Ehrenfeld, J. (1997) 'Organizing for effective environmental design', Business strategy and the environment, 6 (4), pp.187-196.

Lewin, K. (1946) 'Action research and minority problems', Journal of Social Issues, 2 (4), pp.34-46.

Lindahl, M. (2006) 'Engineering designers' experience of design for environment methods and tools - Requirement definitions from an interview study', Journal of Cleaner Production, 14 (5), pp.487-496.

Linstone, H.A. and Turoff, M. (1975) The Delphi method: Techniques and applications. Reading, MA: Addison-Wesley.

Linstone, H.A. and Turoff, M. (2011) 'Delphi: A brief look backward and forward', Technological Forecasting and Social Change, 78 (9), pp.1712-1719.

Lloyd, S. (2012a) Estimate of annual $\mathrm{CO}_{2}$ emissions from defence sector engines in use. Internal report, Rolls-Royce.

Lloyd, S. (2012b) How to manage technical risk with DFMEA. Internal report, RollsRoyce.

Lloyd, S., Lee, J., Clifton, A., Elghali, L. and France C. (2011) The likelihood of restrictions in the availability of metallic resources. Proceedings of the 2011 Engineering Doctorate Conference, University of Surrey, Guildford, UK, 21-22 June. pp.283-297.

Lloyd, S., Lee, J., Clifton, A., Elghali, L. and France, C. (2012a) 'A framework for environmental risk management', The Aeronautical Journal, 116 (1183), pp.941-961.

Lloyd, S., Lee, J., Clifton, A., Elghali, L. and France, C. (2012b) 'Ecodesign through environmental risk management: A case study in critical materials', in: Matsumoto, $M$. et al. (eds.) Design for innovative value towards a sustainable society. Proceedings of Ecodesign 2011 International Symposium, Kyoto, Japan, 30 November-2 December 2011. Dordrecht: Springer. pp.374-379.

Lloyd, S., Lee, J., Clifton, A., Elghali, L. and France C. (2012c) 'Recommendations for assessing materials criticality', Proceedings of the Institution of Civil Engineers - Waste and Resources Management, 165 (4), pp.191-200.

Löfstedt, R. and Renn, O. (1997), 'The Brent Spar controversy: an example of risk communication gone wrong', Risk Analysis, 17 (2), pp.131-136.

Lofthouse, V. (2006) 'Ecodesign tools for designers: defining the requirements', Journal of Cleaner Production, 14 (15/16), pp.1386-1395.

Luttropp, C. and Lagerstedt, J. (2006) 'Ecodesign and the ten golden rules: generic advice for merging environmental aspects into product development', Journal of Cleaner Production, 14 (15/16), pp.1396-1408.

Marsh, P. (2010) 'Rolls-Royce sounds alarm over Reach rules', The Financial Times, 24 June.

Martelli, A. (2001) 'Scenario building and scenario planning: State of the art and prospects for evolution', Futures Research Quarterly, 17 (2), pp.57-74. 
Matten, D. (1995) 'Strategy follows structure: Environmental risk management in commercial enterprises', Business Strategy and the Environment, 4 (3), pp.107-116.

Maxwell, D. and van der Vorst, R. (2003) 'Developing sustainable products and services', Journal of Cleaner Production, 11 (8), pp. 883-895.

McNiff, J. and Whitehead, J. (2000) Action research in organisations. London: Routledge.

Mintzberg, H. (1989) Mintzberg on management. New York: The Free Press.

Mont, O. and Tukker, A. (2006) 'Product-service systems: Reviewing achievements and refining the research agenda', Journal of Cleaner Production, 14 (17), pp.14511454.

Morley, N. and Eatherley, D. (2008) Material security: Ensuring resource availability for the UK economy. Chester: C-Tech Innovation Ltd.

National Research Council (2008) Materials, Critical materials and the US economy. Washington, DC: National Academies Press.

Neumayer, E. (2000) 'Scarce or abundant? The economics of natural resource availability', Journal of Economic Surveys, 14 (3), pp.307-335.

O'Hare, J.A. (2010) Eco-innovation tools for the early stages: an industry based investigation of tool customisation and introduction. Unpublished $\mathrm{PhD}$ Thesis, University of Bath, Bath, UK.

O'Toole, J. (2009) Generic design practice: overview of steps, process and tools used within the Rolls-Royce design process. Internal publication, Rolls-Royce.

Owens, J. (1997) 'Life-cycle assessment in relation to risk assessment: an evolving perspective', Risk Analysis, 17 (3), pp.359-365.

Payne, M. (2001) Health safety and environment process selection system. Internal report, Rolls-Royce.

Penner, J.E., Lister, D.H., Griggs, D.J., Dokken, D.J. and McFarland, M. (eds.) (1999) Aviation and the global atmosphere. Intergovernmental Panel on Climate Change. Cambridge: Cambridge University Press.

Pennington, D.W., Potting, J., Finnveden, G., Lindeijer, E., Jolliet, O., Rydberg, T. and Rebitzer, G. (2004) 'Life cycle assessment Part 2: Current impact assessment practice', Environment International, 30 (5), pp.721-739.

Phillips, C. (2008) Life cycle management and design for environment within RollsRoyce. External presentation, Rolls-Royce.

Pitblado, R. and Turney, R. (1996) Risk assessment within the process industries. $2^{\text {nd }}$ Edition. London: The Institution of Chemical Engineers.

Pojasek, R.B. (2011) 'Linking sustainability to risk management', Environmental Quality Management, 21 (2), pp.85-96.

Pollard, S.J., Yearsley, R., Reynard, N., Meadowcroft, I.C., Duarte-Davidson, R. and Duerden, S.L. (2002) 'Current directions in the practice of environmental risk assessment in the UK', Environmental Science and Technology, 36 (4), pp.530-538.

Power, M. (2004) The risk management of everything. London: Demos. 
Power, M. (2009) 'The risk management of nothing', Accounting, Organizations and Society, 34 (6/7), pp.849-855.

Preston, H. (2010) 'Thames' engine risk management plan. Internal report, RollsRoyce.

Preyssl, C. (1995) 'Safety risk assessment and management - the ESA approach', Reliability Engineering and System Safety, 49 (3), pp.303-309.

Projectsigma (2003) Sigma guidelines to sustainability issues. Available at: www.projectsigma.co.uk (accessed 01-04-2012).

Purdy, G. (2010) 'ISO 31000:2009 - setting a new standard for risk management', Risk Analysis, 30 (6), pp.881-886.

RAEng (2003a) Common methodologies for risk assessment and management. London: Royal Academy of Engineering (RAEng).

RAEng (2003b) The societal aspects of risk. London: Royal Academy of Engineering (RAEng).

RCEP (1998) Twenty first report: Setting environmental standards. London: Royal Commission on Environmental Pollution (RCEP).

RCEP (2002) Special report: The environmental effects of civil aircraft in flight. London: Royal Commission on Environmental Pollution (RCEP).

Rebitzer, G., Ekvall, T., Frischknecht, F., Hunkeler, D., Norris, G., Rydberg, T., Schmidt, W.-P., Suh, S., Weidama, B.P. and Pennington, D.W. (2004) 'Life cycle assessment: Part 1: Framework, goal and scope definition, inventory analysis and applications', Environment International, 30 (5), pp.701-720.

Remmen, A., Jensen, A.A. and Frydendal, J. (2007) Life cycle management: A business guide to sustainability. Paris: United Nations Environment Programme.

Renn, O. (1998a) 'The role of risk perception for risk management', Reliability Engineering and System Safety, 59 (1), pp.49-62.

Renn, O. (1998b) 'Three decades of risk research: Accomplishments and new challenges', Journal of Risk Research, 1 (1), pp.49-71.

Robson, C. (1993) Real world research. Oxford: Blackwell.

Rolls-Royce (2007a) 'Mersey' engine training notes. Internal document, Rolls-Royce.

Rolls-Royce (2007b) Risk management 'how to'. Internal report, Rolls-Royce.

Rolls-Royce (2009) Group Quality Procedure - Manage risk. Internal document, RollsRoyce.

Rolls-Royce (2011) 'Thames' engine factsheet. Internal document, Rolls-Royce.

Rolls-Royce (2012) Annual report 2011. London: Rolls-Royce Holdings plc.

Rolls-Royce (2013) Annual report 2012. London: Rolls-Royce Holdings plc.

Rosa, E. (2010) 'The logical status of risk - to burnish or to dull', Journal of Risk Research, 13 (3), pp.239-253. 
Rose, J. (1997) 'Soft systems methodology as a social science research tool', Systems Research and Behavioural Science, 14 (4), pp.249-258.

Rosenau-Tornow, D., Buchholz, P., Riemann, A. and Wagner, M. (2009) 'Assessing the long-term supply risks for mineral raw materials - a combined evaluation of past and future trends', Resources Policy, 34 (4), pp.161-175.

Rowe, G. and Wright G. (1999) 'The Delphi technique as a forecasting tool: issues and analysis', International Journal of Forecasting, 15 (4), pp.353-375.

Ryan, C. (1999) 'Information technology and DfE: From support tool to design principle', Journal of Industrial Ecology, 3 (6), pp.5-8.

Ryan, C. (2004) 'Learning from a decade (or so) of ecodesign experience, part II', Journal of Industrial Ecology, 8 (4), pp.3-5.

Schein, E. (1990) 'Organizational culture', American Psychologist, 45 (2), pp.109-119.

Schmidt, W-P. and Taylor, A. (2006) 'Ford of Europe's product sustainability index', Proceedings of the $13^{\text {th }}$ CIRP International Conference on Life Cycle Engineering, 31 May-2 June, Leuven, Belgium.

Schwartz, P. (1991) The art of the long view: Planning for the future in an uncertain world. New York: Doubleday.

Sharratt, P.N. and Choong, P.M. (2002) 'A life cycle framework to analyse business risk in process industry projects', Journal of Cleaner Production, 10 (5), pp.479-493.

Simmons, R. (2001) 'Questionnaires', in: Gilbert, N. (ed.) Researching Social Life. $2^{\text {nd }}$ Edition. London: Sage. pp.86-104.

Snape, E. (2009) Engineering and Technology function risk management plan. Internal report, Rolls-Royce.

Spangenberg, J.H., Fuad-Luke, A. and Blincoe, K. (2010) 'Design for Sustainability (DfS): the interface of sustainable production and consumption', Journal of Cleaner Production, 18 (15), pp.1485-1493.

Sroufe, R., Curkovic, S., Montabon, F. and Melnyk, S.A. (2000) 'The new product design process and design for environment', International Journal of Operations \& Production Management, 20 (2), pp.267-291.

Stake, R.E. (1995) The art of case study research. London: Sage.

Steen, B.A. (2006) 'Abiotic resource depletion: Different perceptions of the problem with mineral deposits', International Journal of Life Cycle Assessment, 11 (1), pp.49-54.

Stirling, A. (1998) 'Risk at a turning point?', Journal of Risk Research, 1 (2), pp.97-109.

Stringer, E. (1999) Action research. $2^{\text {nd }}$ Edition. Thousand Oaks: Sage.

Swarr, T.E., Legarth, J.B. and Huang, E.A. (1999) 'Implementation of design for environment at a diversified OEM'. Proceedings of the 1999 IEEE Symposium on Electronics and the Environment, 11-13 May, Danvers, MA, USA.

Taleb, N.N., Goldstein, D.G. and Spitznagel, M.W. (2009) 'The six mistakes executives make in risk management', Harvard Business Review, 87 (10), pp.78-81. 
Tapio, P., Paloniemi, R., Varho, V. and Vinnari, M. (2011) 'The unholy marriage? Integrating quantitative and qualitative information in Delphi process', Technological Forecasting and Social Change, 78 (9), pp.1616-1628.

Tilton, J.E. (1996) 'Exhaustible resources and sustainable development', Resources Policy, 22 (1/2), pp.91-97.

Tilton, J.E. (2003) On borrowed time? Assessing the threat of mineral depletion. Washington, DC: Resources for the Future.

Tilton, J.E. and Lagos, G. (2007) 'Assessing the long-run availability of copper'. Resources policy, $32(1 / 2)$, pp.19-23.

Toyota (2009) Environmentally considerate automobile manufacturing and green purchasing guidelines. Available at:

http://www.toyota.co.jp/en/environment/development/eco-vas/index.html and http://www.toyota.co.jp/en/environment/vision/green/ (accessed 17-02-09).

Undrill, G. (2007) 'The risks of risk assessment', Advances in Psychiatric Treatment, 13 (4), pp.291-297.

US Congress (2010) 'Dodd-Frank Wall street reform and consumer protection act' (PL 111-203, July 21, 2010). United States Statutes at Large 124 (2010) pp.1376-2223.

Van Notten, P.W.F., Rotmans, J., van Asselt, M.B.A. and Rothman, D.S. (2003) 'An updated scenario typology', Futures, 35 (5), pp.423-443.

Van Weenen, J.C. (1995) 'Towards sustainable product development', Journal of Cleaner Production, 3 (1/2), pp.95-100.

Veroutis, A.D. and Fava, J.A. (1997) 'Elements of effective DfE program management and product stewardship', Environmental Quality Management, 7 (1), pp.61-70.

Walsham, G. (1995) 'Interpretive case studies in IS research: nature and method', European Journal of Information Systems, 4 (2), pp.74-81.

Warren, C.A.B. (2001) 'Qualitative interviewing', in: Gubrium, J.F. and Holstein, J.A. (eds.) Handbook of interview research. Thousand Oaks, CA: Sage. pp.83-101.

Weinberg, L. and Eagan, P.D. (1997) 'Introducing design for the environment at Boeing Defence and Space Group through the application of abridged life cycle analysis', Environmental Quality Management, 7 (1), pp.71-79.

Wrisberg, N., Udo de Haes, H.A., Triebswetter, U., Eder, P. and Clift R. (eds.) (2002) Analytical tools for environmental design and management from a systems perspective. Dordrecht: Kluwer.

Yin, R.K. (2003) Case study research: Design and methods. $3^{\text {rd }}$ Edition. Thousand Oaks, CA: Sage. 


\section{Appendix 1: Focus group key-phrase example}

This appendix illustrates how the key-phrase analysis was used to extract results from the focus group data, as described in Section 4.2.3. To demonstrate the analysis, the following pages contains an extended excerpt from the focus group transcripts which is colour coded to show how each part of the transcript was recorded as being relevant to a particular hazard or environmental problem. The initial open coding approach identified these hazards from within the data. Closed coding was then used to reanalyse the data using identified hazards, to provide an appreciation of how often each hazard or topic was discussed and hence how important it might be. The closed coding results are based on how often a hazard or topic was mentioned, rather than the volume of transcript that could be assigned to that hazard.

Colour codes to relate sections of transcripts to a particular hazard are provided in the table below:

\begin{tabular}{|c|c|}
\hline Hazard key-phrase & Colour scheme \\
\hline REACH (use of hazardous substances) & \\
\hline $\begin{array}{c}\text { Access to resources (strategic alloying } \\
\text { elements) }\end{array}$ & \\
\hline Noise & \\
\hline Long-life product & \\
\hline Brand and reputation & \\
\hline
\end{tabular}

The following transcripts illustrate how data was assigned to a key-phrase:

Facilitator: Thank you very much indeed, thank you. OK when you wake up at four am in the morning with a light sweat on your brow what is it you're worried about? I'm talking purely business here.

Respondent: From my point of view business continuity as a result of REACH regulations.

Facilitator: As a result of?

Respondent: REACH regulations.

Facilitator: Yes, can you expand on that?

Respondent: Yes we're coming up towards the November 2010 registration deadline and whilst registration should have been a principle of that visibility and transparency of who's making what or who's importing what it's actually causing companies to make commercial decisions which have very little transparency and visibility down the supply chain to downstream users such as ourselves. So knowing what we cannot buy because no one will make it any more post November is becoming quite tough. 


\begin{tabular}{|c|c|}
\hline Facilitator: & This is because they're not allowed any longer? \\
\hline Respondent: & $\begin{array}{l}\text { No they choose not to register because registration costs } \\
\text { money, a lot of money and governments think industry } \\
\text { have deep pockets and therefore they don't mind putting } \\
\text { a price tag of three hundred and fifty-five thousand euros } \\
\text { on the registration of something but it causes people to } \\
\text { make different decisions to say do we want to make this } \\
\text { any more and without that visibility all we get is worry and } \\
\text { we have to target and [we are] not sort of not sure where } \\
\text { to go. }\end{array}$ \\
\hline Facilitator: & $\begin{array}{l}\text { And that's individual governments as well as euro-wide } \\
\text { governments or? }\end{array}$ \\
\hline espondent: & $\begin{array}{l}\text { Well that particular one is European wide with some } \\
\text { global implications but you know you get a range of things } \\
\text { and yes l've had cold sweats over two whiskeys as well. }\end{array}$ \\
\hline acilitator: & $\begin{array}{l}\text { I think you nodded in sympathy when that first came up, } \\
\text { anything else? }\end{array}$ \\
\hline espondent: & $\begin{array}{l}\text { I did yes, certainly my concern is always business } \\
\text { continuity because I think there's a lot of regulation out } \\
\text { there and growing, increasing number of regulation and } \\
\text { the complexity of it but the concern is always is will that } \\
\text { damage the business? I think they were talking about } \\
\text { immediate, there's short term access to strategic } \\
\text { substances that are being controlled by regulation. }\end{array}$ \\
\hline & $\begin{array}{l}\text { I think then I'm worried from business continuity in terms } \\
\text { of sustainable access to strategic materials for business } \\
\text { as well and I think on the wider sustainability theme is that } \\
\text { it is a real developing theme, a real concern and I think } \\
\text { Rolls like many businesses still hasn't really understand } \\
\text { what sustainability really means to them and how we're } \\
\text { going to gear up for that and how we're going to have } \\
\text { governance around that and how we're responding } \\
\text { publicly to all of that. I think the company is still } \\
\text { somewhat shy when you talk about sustainability and just } \\
\text { for information we are actually working, we've got a } \\
\text { project at the moment where we are trying to develop the } \\
\text { internet RR.com because our web space is really } \\
\text { shockingly poor, we are very conservative in what we say } \\
\text { and do but we are developing that and hopefully it's all } \\
\text { going to a sustainability website. So the company is } \\
\text { starting to move but I think you know in terms of the future } \\
\text { there isn't anything we do that's sustainable. }\end{array}$ \\
\hline Facilitator: & $\begin{array}{l}\text { And this is an environmental problem because if these } \\
\text { things cease to be available? }\end{array}$ \\
\hline espondent: & $\begin{array}{l}\text { We are starting to see it quite quickly now I think on } \\
\text { substances which are starting to be withdrawn from } \\
\text { market because it's the regulator removing them from } \\
\text { market because of their impact either on the environment } \\
\text { or individuals on public health, so that's the driver... }\end{array}$ \\
\hline
\end{tabular}


Continues:

Facilitator: $\quad$ So some not available and some a lot more expensive.

Respondent: Absolutely, well they're expensive because they're scarce but equally you know that you know if you start to see the levels of our development going across the whole world and the world going to nine billion people there just simply is not the resource available and the Chinese are coming in and they're mopping up the Congo and all these areas so these sort of tend to come on difficult areas as well so you foresee increasing difficulty in the next few years of accessing these sorts of substances but yes they are driving price quite high I think in some areas but then there's going to be there's a limit to supplies obviously and then it's like will we have, will it be there, will there be enough to go around?

Facilitator: $\quad$ Sure and what are the alternatives.

Respondent: What are the alternatives and it's things like lithium and stuff like that you know electric cars but there just isn't enough lithium around to do that, so who is going to get the lithium?

Facilitator: OK so that's one problem. Others? Why not, it's your choice, you go.

Respondent: I think from an energy business point we are like an end user, I mean we support the oil and gas industry, we package units, we have derivatives from aero of RB211 and Trent engines and we are putting package units in that really and then we install them in the field in the oil and gas all over the world for heat, power and whatever in platforms and all sorts of things. So if you've got a problem producing the part then obviously we are going to have a problem sustaining the business out there as well because obviously if we can't produce a part or it's different we're going to have to re-engineer what's already out there to suit the supply from your businesses.

Facilitator: And you don't yet know what that re-engineering will consist of because you don't know what's going to be missing except for some extreme cases like lithium.

Respondent: Yes.

Facilitator: Other things?

Respondent: Well going to almost the complete end of the spectrum in terms of the customer facing perspective when I was chatting about this with Stafford my perspective was really 
about product redundancy. We design our products and we have our products out there, if there's any environmental hazards of how tightening legislation actually renders our products redundant, particularly the civil aerospace example would be the fact that jet engines don't have noise regulations you have to put hush kits on them and sometimes it gets to the point where you simply can't fly them and if that curtails our ability to operate and deliver products for the sort of the timescales and lifespan that we'd expected it's a very long term environmental hazard but there's sort of certainly something probably looking in the ten to twenty year horizon where you can sort of start to see environmental legislation that we simply haven't anticipated that makes our actions, to draw on your points, unsustainable and also sort of socially unacceptable, whether it's sort of legislating and unsustainable or whether it's just simply customers will no longer be able to sort of palate the footprint we have in delivering, in operating those products.

Facilitator: $\quad$ Sure, forgive me I may ask one or two daft questions en route, let's hope they can be productive by chance. Surely, I don't know how many aero engine manufacturers of comparable size there are to yours but presumably the problems you face everybody will face, it's the entire industry that's facing the problem. In other words you and your competitors are all up against it to the same extent at the same time.

Respondent: Yes we're all involved in it together in also helping to formulate what those new legislation rules are so as we speak we're actually involved in an inter-community group with lots of bodies, not just OEM producers but also air framers and everybody now and so well what are the potential legislation scenarios and what benefits to the population but what's also the cost. So for each scenario we are trying to understand ideally is there a kicking point up where beyond a certain point you can say it's just economically not viable and one of our interests is basically to look at our own product range to see for each scenario are we in a safe place and are we supporting a level of legislation that's not, that is aggressive to be seen to be doing the right thing but not so aggressive that it hampers our potential, the longevity of our fleet, our future products.

Facilitator: Sure, sure, yes.

Respondent: And one example might be say Barack Obama's recent ban on deep water drilling, that's very sort of you would expect that decision to be over quite a long horizon, we've seen a snap decision very quickly sort of saying right, no deep water drilling in the Gulf of Mexico. It's a massive market where energy would expect to sell a lot of products and normally you sort of, you expect to sort of see that coming in the civil aerospace market it's normally sort of rounds of discussion and sort of about what is a 
reasonable sort of tightening of the regulations, here it's been

Facilitator: $\quad$ And it's a matter of years.

Respondent: Yes well

Respondent: We can all have our inputs to what we think is reasonable but at the end of the day it can be states and governments that just have a clamp down.

Facilitator: Sorry can I just interrupt one minute and then please continue, am I right in understanding what is happening now is that there is an industry wide attempt to foresee what is coming.

Respondent: Or to define it.

Facilitator: Define it and see how the industry as a whole will cope but within that of course you have your own competitive push.

Respondent: Underlying all of that.

Facilitator: $\quad$ As do everybody.

Respondent: Yes, everyone's got the same position, the same environment, it's not competitive of course but really underneath we actually still need to maintain our own product portfolio and longevity of it.

Facilitator: $\quad$ Yes indeed, OK sorry I interrupted you.

Respondent: No I think that's covering a lot of what I was saying and I think most of our industries we see that sort of incremental tightening and that's something that we're in a position to respond to fairly well, what worries me is that decision or that tightening or sort of those events that happen which suddenly change the perception of what we as a company do in any of our sectors. So I mean the energy example is just the most recent but if there's sort of step changes in sort of environmental legislation that or just perception of our environmental footprint that sort of really radically affects our business then it's a hazard, it might be ten or twenty years out but and it's a question of what is its relative impact versus in the short term but its

Facilitator: $\quad O K$.

Respondent: I think also now we have spin offs from that because that does happen obviously and we've got people that we need to train up so it does have some spin offs, wider spin offs of how many people do we need as a resource to manage the business because if that's something you've got to weigh we are either over-staffed or if we don't be aware of these issues we could actually under-staff and at the minute we're probably running shortfall on service out in the operation team operating where we are today and the energy business as we've said earlier hopefully is 
going to double their output in the next five years. So we've got to plan our resource to manage that for the after sales and things like that.

Facilitator: $\quad$ OK so starting from both numbers and time.

Respondent: Yes.

Respondent: I think it's worth making the point Michael as well that there's two distinct areas here. I think you've got product issues and then you've got operations issues and supply chain as well and you've got regulation around product we're using that have got a twenty, thirty year lifetime product haven't we rather than a phone that changes every six months sort of thing but in terms of facilities you know you've got increasing regulation there that's starting to impact on where you build facilities and how you build them, how they are benchmarked...

Continues: $\quad$...so there's regulation now with indexes that will in effect can potentially tarnish a brand because you may appear to be performing rather less well than you really are just because of the way regulation's geared in gas companies as it were and we've got a good example there of engine testing you know we need to test engines so we've got lots of people to do that but the massive benefit there is the efficiency saving we're driving for you know that is our biggest contribution to society is producing more efficient products but then you've got to be able to do that but then you get shot in the foot because then you know you're publicly shamed or you could be in the next year or two as increasing carbon footprint so you look like a bad company. So in terms of brand and reputation the company could get tarnished and the board is worried about this, not the fact that there's something called the carbon reduction commitment regulations and it's going to cost us say another million pounds a year in tax, the board's not worried about a million quid in tax, they'll just pay it but they are worried about where we appear in the league table and because of what we do we could go to the bottom that will affect brand, the reputation. So there's these two distinct areas and there's all the product type stuff but then there's all the operation supply chain which start to affect where you decide to do your business.

Facilitator: $\quad$ Yes, OK another variable.

Respondent: May / be allowed?

Facilitator: Yes please, please.

Respondent: To support I think I heard you Nigel I recognised, your voice is easily recognisable.

Respondent: $\quad$ Yes it was me Brit, good morning. 
Respondent: Hi Nigel, I would like to support you Nigel on this and this is also a voice from the traditionally very conservative marine business, even we also see the heat going up both on the production of our products but also on their operational part of our products. I think it's important for this working group to or this focus group to be aware that there are two lines we need to go down here, two roads we need to go down and both of these are equally important and we see our customers and also we see the regulating bodies interested in both these paths for our products. So I think I would like to support you on that Nigel that we need to be able to have two thoughts in their heads at the same time here.

Respondent: Yes marine's got a much bigger footprint than aviation and you see it start, public interest is starting to swing, the regulatory interest is starting to swing now to marine particularly in coastal waters where you've got air quality issues as well so they've sort of been getting away with it for a while so to speak. Of course the bulk of stuff is transported by the marine environment, aviation is small in comparison.

Facilitator: Is that increased concentration on the problem, is it a spin off of things like BP?

Respondent: I don't think it's BP I think you know it's all part of the climate change debate.

Facilitator: But that won't have helped it I guess?

Respondent: No if you were to look at an environmental example in marine the Exxon Valdez disaster actually was a big impact on the marine business, not necessarily Rolls Royce but the broader marine business because you went from people saying we have single shell takers with just one skin to having double skin tankers basically for that extra margin of safety. It actually then drove a complete re-fleeting of the oil tanker market because of that single disaster, that would be sort of probably another sort of thing where you sort of see a radical disaster on the product side then driving through, I just think the problem is beneficial to the industry because it drove sort of new orders and new volume but at the same time for the sort of the MGs they just go oh l'll buy new ships, so sort of a big environmental disaster had a major impact on the end product. 


\section{Appendix 2: Published papers}

This appendix contains the following papers published by the researcher. Papers are presented in chronological order of publication, starting with the most recent:

1. Recommendations for assessing materials criticality (Lloyd et al., 2012c).

Published in the Proceedings of the Institution of Civil Engineers (ICE) Waste and Resources Management, as part of a special issue on critical materials. This paper presents recommendations on how material criticality assessments can be improved, including a framework for completing criticality assessments. This paper won a Telford Premium award, acknowledging papers of exceptional quality published in ICE journals.

2. A framework for environmental risk management (Lloyd et al., 2012a).

Published in The Aeronautical Journal, this paper presents the framework for environmental risk management, aimed at a practitioner audience. How the framework could work is also discussed through two examples.

3. Ecodesign through environmental risk management: $A$ focus on critical materials (Lloyd et al., 2012b).

Published in the Proceedings of the Ecodesign 2011 International Symposium, held in Kyoto, Japan. This paper is based on an adaptation of the first case study presented in chapter 5 , showing how the risk based approach to DfE can work.

4. The likelihood of restrictions in the availability of metallic resources (Lloyd et al., 2011).

Published in the Proceedings of the 2011 Engineering Doctorate Conference, this paper attempts to develop and test a method for assessing the likelihood that materials might be affected by supply restrictions. 
Waste and Resource Management Volume 165 Issue WR4

Recommendations for assessing materials criticality

Lloyd, Lee, Clifton, Elghali and France
Proceedings of the Institution of Civil Engineers Waste and Resource Management 165

November 2012 issue WR4

Pages 191-200 http//dx.doi.org/10,1680/warm. 12.00002

Paper 1200002

Received $01 / 03 / 2012 \quad$ Accepted 01/05/2012

Keywords: natural resources/reviews/sustainability

\section{Recommendations for assessing materials criticality}

Stafford Lloyd MEng, AiEMA

Research Engineer, Rolls-Royce, Bristol, UK, and University of Surrey, Guildford, UK

Jacquetta Lee MEng. PhD

Tutor, University of Surrey, Guildford, UK

Andrew Clifton BEng, PhD

Manager, Sustainable Development, Rolls-Royce, Derby, UK
Lucia Elghali MSc, EngD, AIEMA

Senior Lecturer, University of Surrey, Guildford, UK

Chris France MSC, PhD, FRSA

Professor, University of Surrey, Guildford, UK

This paper provides recommendations for assessing the criticality of materials (metals and non-fuel minerals), including the need for context-dependent assessment methods, providing a framework for conducting criticality assessments. Materials criticality captures concerns over the accessibility of materials, as the product of assessing a material's 'supply risk' and the impact of a supply restriction. Through a review of selected studies, problems with criticality assessments are discussed, highlighting how these become particularly important when the results of assessments are used in decision making. Considering how the results of criticality assessments are used in decision making highlights how criticality exhibits some of the characteristics of a 'complex context'. Building on predefined attributes of effective decision support in complex contexts, recommendations are made on how these problems can be addressed to better assess criticality in the future. These also include building on metric-based assessment methods by developing scenarios of future material supply and demand.

\section{Introduction}

Concerns over the availability of materials have resurfaced recently, owing to changes in the demand patterns of raw materials from the emergence of new technologies, developing lifestyles, market dynamics and government policies (Erdmann and Graedel, 2011). The increasing interest in materials criticality is encouraging, as it is an important and complex problem. However, it does have the potential to create confusion over what materials are considered 'critical', or even what term should be used to describe the phenomenon. The purpose of this paper is to provide clarity, by defining the precise problem, the term that should be used to describe it and how it should bc assessed. The aim of the paper is not to offer a new methodology for assessing criticality; there are already more than a dozen studies on this aspect (Erdmann and Graedel, 2011) and offering a new approach would only add to the potential for methodological confusion.

Section 2 clearly defines what is meant by "materials criticality" in an attempt to clarify what the problem is and to settle on a term to describe it. A concise review of selected methods for assessing criticality is given in Section 3, providing a summary of metrics commonly used. The review is used to highlight problems with criticality assessment methods developed to date, and more generally with assessing criticality as described. Section 4 discusses how these problems become particularly important when the results of criticality assessments are used within decision making, highlighting how criticality exhibits some of the characteristics of a 'complex context'. Building on predefined attributes of effective decision support in complex contexts, Section 5 provides recommendations on how criticality can be assessed better in the future, including a framework for completing criticality assessments. Conclusions are given in Section 6

\section{Materials criticality}

Until the middle of the last decade, concerns over the availability of non-renewable raw materials (metals and non-fuel minerals) focused on the problem of geological scarcity (Erdmann and Graedel, 2011). Efforts to understand the magnitude of the problem applied measurements based on depletion indices (Lee, 1998), which calculated the years of resource remaining by dividing a measure of the amount of resource left by its rate of consumption (Cohen, 2007; Tilton, 2003). Typically, indices used either static values of resources availability or applied dynamic assessments (Alonso ef al., 2007).

While this appears to be simple, the problem is significantly more complex, as any estimates of the amount of a resource left contain significant uncertainties. From Gordon ot al. (2007), cstimates can be based either on resources, reserve base or reserves. Resources are, effectively, the feasibly available amount of a matcrial in the Earth's crust. Reserve base represents resources that are decmed to be extractable with 
Recommendations for assessing materials

criticality

Lloyd, Lee, Clifton, Elghali and France current technology. Reserves are the economically extractable parts of the reserve base at current prices. Estimates of reserves and the reserve base are governed by the potential for profitable extraction at ally particular time, making the data very unrcliable (Gordon et al., 2007).

To a certain extent, these uncertaintics have led to a continuing debate on the availability of materials, which remains polarised between those who see depletion as a significant issue and those who have faith in technology and free markets. A view of the main arguments is availablc from a varicty of sources; see for example Tilton (1996), Neumayer (2000), Steen (2006), Tilton and Lagos (2007), Gordon et al. (2007), and Morley and Eatherley (2008).

More recent studies suggest that the debate is moving on, adopting a new perspective and revealing a different problem. As pointed out by Rosenau-Tornow et al. (2009), and others, the question is increasingly becoming one of accessibility rather than availability. Historical examples suggest that the accessibility of resources can be affected by economic, social and environmental constraints as well as geological ones. The 'cobalt crisis' in the 1970s was due to conflict in Zaire (now the Democratic Republic of the Congo), a key supplying region (Alonso et al., 2007). Recent interest in the topic of materials accessibility has been engendered by China dominating supplies of rare earth elements, which geologically are not that rare (National Research Council, 2008). So the question is whether or not the accessibility of resources will be affected by economic, social, environmental and geological constraints and, if so, what might be the consequences.

Presenting what is effectively a risk assessment, materials criticality seeks to understand this problem by assessing the likelihood of a material supply restriction (often termed 'supply risk') and the potential impact if a restriction were to occur. Capturing these two measures neatly in the "criticality matrix' (Figure 1), a material is considered critical if it is high risk; that is the likelihood of a supply restriction is high and this would have significant negative consequences if it were to occur.

\section{Assessing materials criticality}

Commonly cited reasons for conducting materials criticality assessments include

- increasing and new demand for materials from developing economies

- reliance of developed nations on imported material commodities, which sometimes come from less stable supplying regions

- demand for a wider range of material inputs from new technologies

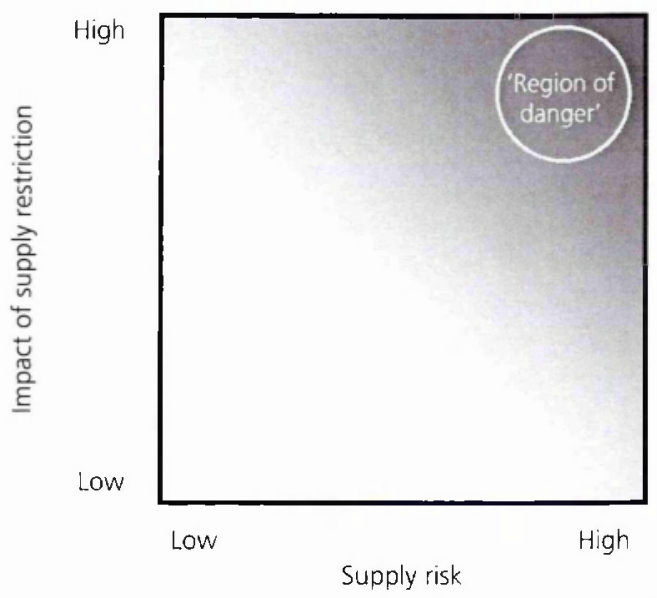

Figure 1. The criticality matrix (National Research Council, 2008)

- recognition of the social and cnvironmental consequences of extraction

- national policy measures that have the potential to disrupt the operation of global markets

- concentrations of production, creating supply monopolies.

Most criticality assessment methods adopt the matrix as a framework, applying metrics to assess supply risk and the impact of a supply restriction, and aggregating the results to identify materials that are considered critical. Metrics for assessing supply risk typically reflect common concerns about why materials might become restricted (as listed), or are derived from factors that in the past have given rise to disruptions in supply, for example political instability in key supplying regions (as in the "cobalt crisis"). Assessments of impact depend on the context within which the study is being performed, although they can adopt either a global, national or company perspective (Graedel et al., 2012). Aggregation is typically done by translating scores into a common scale, sometimes with weighting factors being applied to highlight the contributions of metrics deemed to be more significant. Results are then plotted on to the matrix to identify critical materials.

A summary review of key criticality assessment methods is presented in Table 1 and discussed in the following subsections. The review is not intended to be exhaustive; an in-depth analysis of the literature is already available (Erdmann and Graedel, 2011). Instead, the purpose is to review selected methodologies to highlight problems with how criticality has been assessed to date and hence what can be done to improve methods in the future. From the numerous studies available for review, five were selected (to provide an overview of the assessment methods developed), on the basis of frequency of citation in the literature (European Commission, 2010 ('EU study'); Morley and Eatherley, 2008 ('Oakedene Hollins study'); National Research 


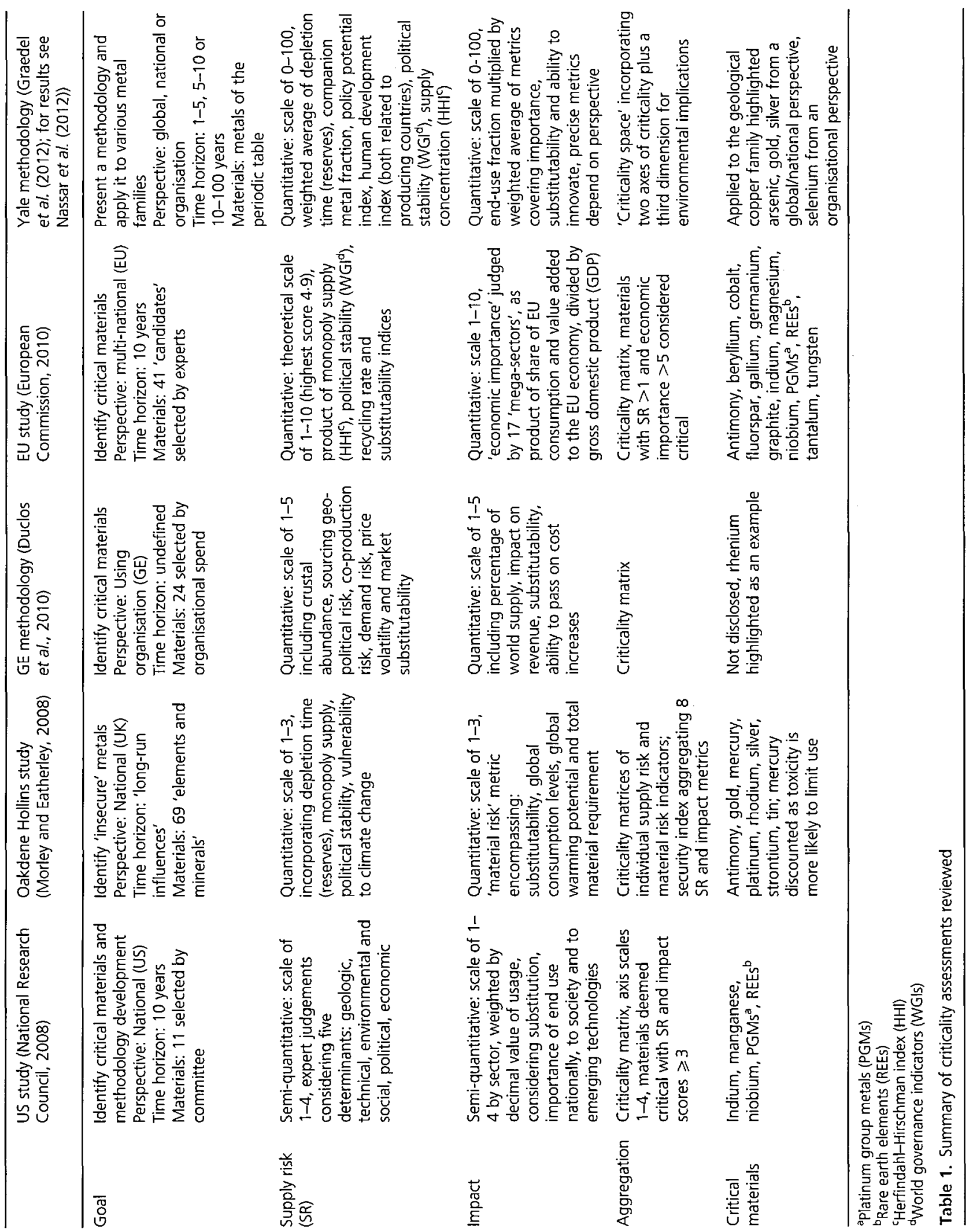


Council, 2008 ('US study') or their relevance to the UK (European Commission, 2010; Morley and Eatherley, 2008). One study was selected as it provides a perspective from a using organisation, General Electric (GE) (Duclos et al., 2010 ('GE methodology'). Another, developed at Yale university, was chosen because it was the most recent and was also deemed to present the most robust methodology developed to date (Graedel et al., 2012 ('Yale methodology'). Three methodologies that only assess supply risk were overlooked (AEA Technology, 2010; BGS, 2011; Rosenau-Tornow et al., 2009), as strictly speaking a material could have a high supply risk and not be critical. Other studies were overlooked either because a version in English was not available or because the studies were not deemed to cover anything not included in those being reviewed.

\subsection{Study goals}

All of the studies reviewed aimed either to identify critical materials and/or to present a methodology that could be used to highlight materials that might be critical. Lists of critical materials are presented by the Oakdene Hollins and EU studies to make recommendations for mitigating actions, which include the need for further investigation and policies to mitigate potential supply restrictions. The GE study researchers sought to use their methodology to identify steps that can be taken to mitigate the risks. The US study was more hesitant, explicitly stating that its goal was not to offer a discrete list of critical materials, as it was only a preliminary study. Its position on not developing a critical materials list highlights how uncertainties in assessments require caution when results are used within decision making, which supports later discussion.

All of the studies adopted a slightly different perspective, time horizon or focused on different materials. The perspective depends on the system under study, which, in turn, will influence the materials selected. Time horizons were selected to strike a balance; too short and it can be difficult to separate short-term supply and demand fluctuations from what might be more fundamental problems with accessibility. Too long and it becomes almost impossible to predict problems with any degree of certainty. Both the US and Yale studies suggest that the nature of the assessment should depend on the time horizon adopted.

\subsection{Supply risk, impact and aggregation}

Each of the reports reviewed applied a different set of metrics for measuring supply risk and impact, aggregating them in different ways. The following subsections review the main methodological considerations.

\subsubsection{Supply risk}

Most reports covered all of the five determinants initially identified by the US study in assessing supply risk: geological, technical, political, environmental and social, and economic.
Geological measures were explicitly excluded by the EU study, being deemed unreliable in predicting long-term availability and irrelevant in the short to medium term. Morley and Eatherley (2008) also considered geological measures of secondary importance owing to their uncertainty. Caution should certainly be applied when using geological measures within criticality assessments (Lloyd et al., 2011), although they should not necessarily be excluded. Graedel et al. (2012) suggest that depletion times give a useful indication of the current relationship between supply and demand, and viewed in this way they are a useful measure. In the longer term (more than 10 years), economic and social factors become very difficult to predict and Graedel et al. (2012) suggest that only geological measures for assessing supply risk should be used in this timeframe, combining reserve base estimates with component metal fraction. Component metal fraction, or 'coproduction risk' as described in the GE method, reflects the fact that some materials are produced from traces within the ore of a 'host' metal. For example, hafnium is largely produced as a co-product of zirconium, which comes primarily from titanium ores. As a co-product, the supply of material is governed by demand for its host, not for the material itself, so there is no guarantee that supply will change if demand does. Discussions with commodity experts suggested that co-production is a valid and important indicator of supply risk.

Monopoly supply was a metric adopted by all studies as a clear indicator of potential material supply restrictions. The most popular, and probably most robust, means of assessing monopoly supply is the Herfindahl-Hirschman index (HHI), which, in its simplest form, is the sum of the squares of the supply percentages of producing countries, producing a theoretical value of between 0 and 10000 . (For example, if $40 \%$ of material $\mathrm{X}$ is produced in country $\mathrm{A}, 40 \%$ in country $\mathrm{B}$ and $20 \%$ in country $\mathrm{C}$, the $\mathrm{HHI}$ would be $40^{2}+40^{2}+20^{2}=3600$. A higher HHI score indicates a greater risk of supply restrictions.) This value is usually adjusted to reflect the scoring scheme applied by an assessment. While this is a relatively simple measure, it is still necessary to decide the level of supply concentrations that should give cause for concern. Alonso et al. (2007) suggest that high levels of concern exist when market shares reach $40 \%$. A HHI of 1800 is considered problematic by the US Department of Justice (Graedel et al., 2012). Monopoly supply conditions become more of a concern when concentrations occur in less stable regions, so studies tend to merge HHI scores (or other measures of supply diversity) with an assessment of political stability. Two prominent indices are the world governance indicators (WGIs) (Kaufmann et al., 2010) and the failed states index (FSI) (Messner, 2011). Both analyse a variety of social indicators to produce scores indicating politically stability. As with the HHI, these scores are usually manipulated to be consistent with the scoring scheme used by an assessment. Given the WGI and FSI are both 
single scores aggregated from a wide variety of metrics, further manipulation could create meaningless numbers.

Other popular factors for assessing supply risk include recycling rate, substitutability, price volatility, demand changes, and environmental and social indicators. Availability of secondary (i.e. recycled) materials reduces dependence on primary production, hence reducing the risk of supply disruptions. Economic data on recycling rates were used in the EU report, presented by material as a decimal. Improved data on the percentage recycling rates of materials have now been developed (Graedel et al., 2011).

Substitutability appears in both the measures of supply risk and impact. As a factor of supply risk it considers all uses of a material. If the material lacks substitutability in all or the majority of its applications, restrictions in supply will be emphasised as mitigating options will be limited. In the EU report substitutability was presented as a decimal similar to recycling rates, determined through expert judgement. Other methodologies included it as an impact metric. Price volatility is a useful indicator of supply risk in the short to medium term, as price fluctuations in a material could indicate underlying market dynamics that could lead to supply restrictions. Volatility is often measured as a ratio of maximum to minimum price for a material over a set time period (10 years is common). Demand changes are generally included as new applications create competing uses for a material, potentially restricting supply, in particular if production cannot be increased to meet demand, for example for materials produced as a coproduct.

Environmental and social indicators reflect the fact that these impacts can inhibit (or in the case of social measures, sometimes encourage) primary production. It is considered that environmental limitations are more likely to restrict supply than physical scarcity (Morley and Eatherley, 2008). Many environmental and social metrics can be used and can either be aggregated within an assessment of supply risk, as used in the GE, Oakdene Hollins and US studies, or considered separately, as used in the EU report and the Yale methodology. The EU report applied an additional environmental country risk metric, based on a policy performance rating system (coincidentally also developed by Yale University) applying a distance-to-target approach. The logic is that the further a country is from stated environmental policy goals, the more likely it is to restrict production of materials. Other environmental measures usually apply proxies, such as total material requirement or global warming potential (in carbon dioxide equivalents) measured per kilogramme of material produced. Indices such as the human development index (HDI) can be applied to highlight the presence of social issues within producing countries.
A summary of the various metrics typically used to assess supply risk is provided in Table 2, along with their underlying assumptions.

\subsubsection{Impact}

Measurements of impact are inevitably context dependent and the metrics used typically depend on the perspective of a study. From the perspective of a using organisation, the focus is on the materials that are critical to meeting objectives, whether these are commercial or related to fulfilling critical functions (for example within the military or medical industries); rhenium was highlighted as an issue by GE owing to its importance in the manufacture of gas turbine engines. Metrics tend to focus on issues such as the ease of substitutability, price volatility and potential impact on revenue and product costs.

National perspectives focus more on importance to the economy, as reflected in the EU study, also including factors such as substitutability and environmental impacts. From a global perspective, suggested indicators include importance to using populations and environmental metrics, as well as ease of substitutability. A longer-term focus is also required from a

\begin{tabular}{ll}
\hline Supply risk metric & Underlying assumption \\
\hline $\begin{array}{ll}\text { Geological measures (depletion } \\
\text { indices or abundance) }\end{array}$ & $\begin{array}{l}\text { Low depletion index/ } \\
\text { abundance increases risk }\end{array}$ \\
Co-production & $\begin{array}{l}\text { Primary ore dependence } \\
\text { increases risk }\end{array}$ \\
Monopoly supply & Supply concentrations \\
& increase risk
\end{tabular}

Political stability Instability in producing regions increases risk

Recyclability Lack of recycled sources increases risk

Substitutability

Lack of substitutability increases risk

Environmental impact

High environmental impacts increase risk

Demand changes New demand increases risk

Price volatility

High price volatility increases risk

Social impact

Presence of social issues increases risk

Table 2. Summary of supply risk metrics and underlying assumptions 
global perspective. Graedel et al. (2012) present a comprehensive set of metrics for addressing impact from each perspective, summarised in Table 3.

\subsubsection{Aggregation}

Aggregation is a multi-criteria problem, requiring the need to capture judgements on how a wide-ranging set of indicators and sources of information can be brought together in a meaningful way to highlight critical materials. Combining measures requires translating metrics into a common scale, including weighting factors (if required) to highlight the contributions of metrics deemed to be more significant. Judgements are required to determine the level at which concern should be raised for metrics on their own and when combined within the criticality matrix.

\begin{tabular}{|c|c|c|}
\hline Perspective & Components & Indicators \\
\hline \multirow[t]{3}{*}{ Organisation } & Importance & $\begin{array}{l}\text { Percentage of revenue } \\
\text { impacted } \\
\text { Ability to pass through cost } \\
\text { increases } \\
\text { Importance to corporate } \\
\text { strategy }\end{array}$ \\
\hline & Substitutability & $\begin{array}{l}\text { Substitute performance } \\
\text { Substitute availability } \\
\text { Environmental impact ratio } \\
\text { Price ratio }\end{array}$ \\
\hline & $\begin{array}{l}\text { Ability to } \\
\text { innovate }\end{array}$ & Corporate innovation \\
\hline \multirow[t]{3}{*}{ National } & Importance & $\begin{array}{l}\text { National economic } \\
\text { importance } \\
\text { Percentage of population } \\
\text { utilising }\end{array}$ \\
\hline & Substitutability & $\begin{array}{l}\text { Substitute performance } \\
\text { Substitute availability } \\
\text { Environmental impact ratio } \\
\text { Net import reliance ratio }\end{array}$ \\
\hline & Susceptibility & $\begin{array}{l}\text { Net import reliance } \\
\text { Global innovation index }\end{array}$ \\
\hline \multirow[t]{2}{*}{ Global } & Importance & $\begin{array}{l}\text { Percentage of population } \\
\text { utilising }\end{array}$ \\
\hline & Substitutability & $\begin{array}{l}\text { Substitute performance } \\
\text { Substitute availability } \\
\text { Environmental impact ratio }\end{array}$ \\
\hline
\end{tabular}

Table 3. Metrics for assessing the impact of a supply restriction (Graedel et al., 2012)
All assessment methods applied different methods for aggregating metrics to give scores of supply risk and impact, in turn, using different matrices to assess criticality. Very few studies justify the aggregation approach, either in terms of how common scales were developed or boundaries of significance chosen, providing very little transparency on the reasoning behind decisions made. This makes the results difficult to defend as a robust assessment. It might be the case that aggregation in some studies is governed more by the need for ease of numerical manipulation, rather than analytical rigour, which would lead to more robust results (Erdmann and Graedel, 2011).

\subsection{Problems with criticality assessments}

There are several problems with some of the criticality assessment methods reviewed and more generally with assessing criticality as described. It is clear that assessments of criticality depend upon subjective judgements, at various levels. Metrics used to assess supply risk and impact are inevitably influenced by the perspective of the system being studied, materials being assessed and the time horizon. Some metrics themselves may be dependent on subjective interpretations, for example substitutability. Value judgements are also implicit in any weightings used to highlight metrics that are more or less significant, how the metrics are aggregated and where boundaries of significance are set to identify materials that are more or less critical. It is inevitable that different studies will arrive at different results and these subjectivities make it unlikely that the results of any one assessment method can be universally applied.

Criticality assessments also contain significant sources of uncertainty, which are rarely addressed. Results of an assessment are often dependent on the aggregation of diffuse metrics, derived from incomplete data or qualitative judgements. Applying aggregation methods means that small changes in underlying metrics, which are inherently uncertain, can significantly influence results (Erdmann and Graedel, 2011; Lloyd et al., 2011). From the assessment methods reviewed, only the Yale method addressed uncertainty. The suggested approach was to conduct Monte Carlo simulations using distributions modelling variations in metrics, tracking how these affect key results when aggregated (Graedel et al., 2012).

In all assessments uncertainties need at least to be acknowledged, or better, understood to some extent through suitable analysis. At the least, methodologies should be developed iteratively, reviewing initial results and adjusting the methods appropriately to ensure they reflect underlying concerns. However, all studies lack explanation of how the underlying methodological choices lead to the results obtained and whether or not they are appropriate.

As discussed in the critique of aggregation methods, there is also a lack of transparency within assessment methods. All 
studies and methodologies are dependent on decisions regarding the metrics to be used, how they are aggregated and how critical materials are identified. However, most offer little justification for the methodological choices made. Organisations concerned about critical materials will want to run their own assessments, although even to begin with it is often very difficult to take an existing study and re-create results.

Data challenges present further difficulties for conducting criticality assessments. Data can be difficult to obtain in the first place, for example production data on co-products may be privately held. Indices for providing an indication of political stability, or databases containing information on the environmental impacts of production, may be proprietary and lack transparency. Disagreements between sources of data are also not uncommon, for example discrepancies between production data compiled by different national geological surveys. Managing the sheer amount of data required can represent a challenge in itself, especially if information needs to be updated on an annual basis.

A lurther problem, which is often overlooked, is that some of the methodological decisions are influenced by past events. Selecting metrics is often done on the basis of what has historically caused supply disruptions. Some metrics are actually records of past data (c.g. price volatility). Judgements on boundaries of significance can also be dependent on what has caused problems in the past (e.g. levels of supply concentration). Criticality assessments should seek to look into the future, and experience suggests that using historical precedents is a poor means of doing so (Taleb et al., 2009).

\section{Implications for decision making}

Some of these problems are inevitable and most studics acknowledge that the nature of criticality assessments will differ, depending on who they are developed by and for what purpose. The EU study noted the diverse outcomes from assessments performed at a national level by member states, in particular how differences in national economies affected perceptions of economic importance. Subjectivitics are also acknowledged within the Yale methodology, which explicitly seeks to 'permit flexibility by the user in its application' (Graedel et al., 2012). However, these problems tend to be overlooked when they become important, which is when the results of criticality assessments arc used to agree mitigating actions, whether at an organisational. industry, national or supranational level (for example the EU). There is a perceived tendency to create lists of critical materials and apply them to mitigate risks unilaterally, with little consideration for the suitability of the methods that have derived them and what the implications of this might be for materials that may actually be more or less critical.
Lack of consideration for the context-dependent and uncertain nature of criticality assessments within decision making could lead to the incorrect signals and behaviours. The authors have seen examples of policy actions made using criticality lists, which do not reflect the concerns of a particular organisation or even industry. It is very unlikely that de facto lists of critical materials can be simply developed and applied to mitigate all risks, as the value-laden nature of assessment methods means that a universal set of critical materials cannot be developed. Criticality is far too much of a complex problem to be addressed in this way, although considering complexity does lead to some potential solutions to these problems.

Considering how the results of criticality assessments are used in decision making highlights how criticality exhibits some of the characteristics of a 'complex context', discussed by Elghali et al. (2008) as a common feature of decisions that involve evaluations related to sustainability. In particular, materials criticality (from Elghali et al., 2008)

- is characterised by a dynamic system of problems with emergent properties that could not be produced by one part of the system alone

- involves social aspects that need to be adequately considered alongside technical ones

- requires different interpretations of the problem dependent on the context

- has a degrec of 'post-normality', where values guide outcomes as well as scientific certainty.

Elghali et al. (2008) also identify attributes of effective decision support in complex contexts, stating that any approach, among other things, must

- explicitly include considerations for uncertainty arising from incomplete information

- involve all relevant stakeholders within decision making, in particular those who can provide first-hand experience of a problem

- rccognise multiple criteria and how they contributc to an assessment

- be adaptable to changing situations and outcomes, providing an audit trail of decisions made and why.

\section{Recommendations for assessing criticality}

Building on these attributes of effective decision support in complex contexts, the following recommendations arc made to improve assessing materials criticality in the futurc.

Reflecting the need to explicitly include uncertainty and recognise multiple criteria, criticality assessments need to provide a transparent view of how results are affected by methodological choices and uncertaintics in underlying metrics, which also need 
Recommendations for assessing materials

criticality

Lloyd, Lee, Clifton, Elghali and France to be appropriately acknowledged within decision making to determine any mitigating actions. Assessments should also be accompanied by a discussion of how the methods chosen have led to the results obtained and whether or not they are sensible given underlying concerns. For example, if a material is highlighted as critical owing to its geological scarcity, but because of inherent uncertainties in the data this is considered of secondary importance, one action could be to alter the assessment method with a lower weighting for geological metrics.

To involve all relevant stakeholders and be adaptable to changing situations, and acknowledging the subjectivities of criticality, context-dependent assessment methods are required, developed through the use of a stakeholder community. Such a community should include those with first-hand experiences of the problem, for example purchasing professionals. It would be impractical if every organisation performed its own assessment and acted unilaterally to mitigate any risks identified from materials highlighted as critical. However, it is also not possible to develop a global or even national list of critical materials that can be used by everyone. Balance is required between practicality and possibility and it is suggested that performing assessments at the level of an industry sector might provide such a balance. It is likely that companies in the same industry will use the same materials and share concerns over what could cause restrictions in accessibility. Being in the same industry it is also likely that organisations collaborate on other issues, in particular when it comes to influencing policy on, for example, national research funding. Industry groups could be developed that include the necessary stakeholder representation, agreeing a joint assessment method.

To facilitate context-dependent assessments, a transparent, peer-reviewed open-source database of metrics to support criticality assessments is required, incorporating measures of uncertainty. These need to cover the variety of measures required to measure both supply risk and impact, summarised in Tables 2 and 3, drawing information from a variety of sources, for example

v national geological surveys, providing data on geological measures, production and co-product risks

- organisations that produce political stability and social indicators, such as the world governance and human development indices

- economic data, in particular on price volatility and supply and demand patterns

- databases providing information on the environmental impacts of material production.

Alongside metrics, a framework for criticality assessments is required. An example of such a framework is provided in Figure 2, identifying the main steps within an assessment and

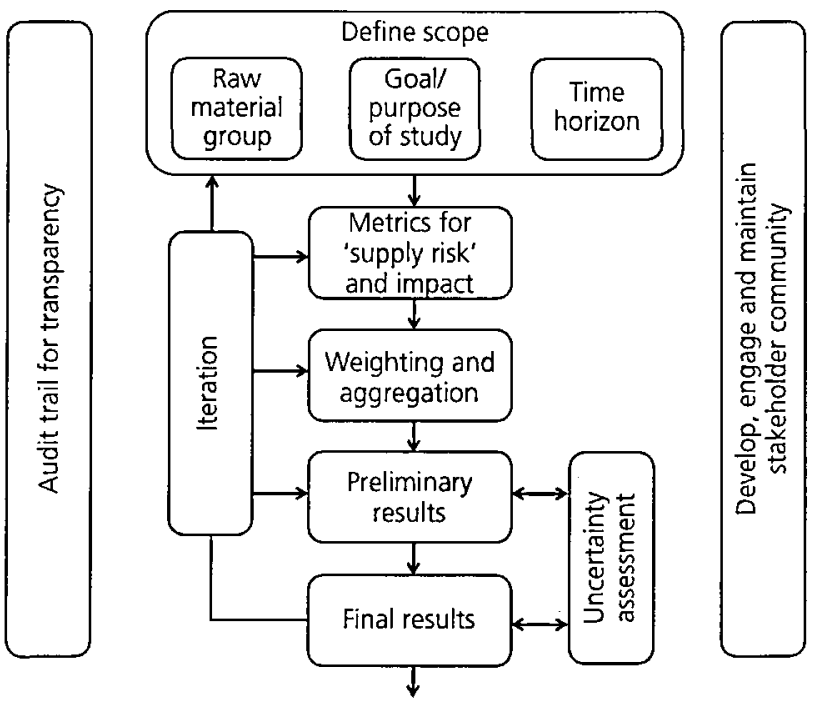

Figure 2. Framework for criticality assessment

highlighting the importance of considering iteration, uncertainty, transparency and engaging with a stakeholder community. Criticality is a dynamic problem, so it is also recommended to update assessments on a regular basis, perhaps annually, to highlight materials that may have become more or less critical.

Finally, it is recommended to build upon criticality assessments that rely on the use of metrics by developing scenarios of supply and demand, to overcome reliance on historical precedents. It should be made clear that scenarios do not seek to predict the future, as this is impossible to do with any degree of certainty (Sohn, 2006). Instead, scenarios seek to develop 'consistent and plausible pictures of possible future realities' (Lempert et al., 2009).

Several studies have already attempted to construct scenarios of material supply and demand; see for example Alonso et al. (2012), Gruber et al. (2011), Kleijn and van der Voet (2010) and Yang (2009). All of these studies attempt to analyse what might happen in terms of material supply and how new technologies and demographics may change demand, analysing implications for materials availability. With such a complex problem, it is inevitably difficult to model changes in material supply patterns and how innovation and demographics may affect demand. However, producing scenarios does provide a more in-depth understanding of the dynamics of material supply systems and hence where restrictions may occur, particularly in the longer term. Some scenario studies do have a tendency to focus on basic measurements of resource quantities, re-focusing on availability, rather than accessibility, as discussed previously. More robust scenarios would seek to capture potential problems in both availability and accessibility. 
As scenarios are just a possibility, they should be treated with care. Convergence between scenarios on materials that are more or less critical could indicate a higher likelihood of longterm problems. One approach could be to use criticality assessments for initial screening, highlighting materials for which a more in-depth scenario analysis would be beneficial.

\section{Conclusions}

Materials criticality is a complex problem that has only become a topic of serious interest in the last 5 years. Given this complexity, limitations in assessment methods are inevitable and most of the problems highlighted in this paper have been acknowledged in previous studies and reports. However, from the authors' experiences, where these problems tend to be overlooked is when the results of criticality assessments are used in decision making, which is also when they become the most important. Not addressing these problems could lead to the wrong signals and behaviours, potentially increasing the risks posed to businesses and the economy instead of mitigating them.

Through drawing on attributes of decision support in complex contexts, this paper has made recommendations on how these problems can be overcome, including the following points.

- Transparency should be provided within assessment methods so it is clear how the methodological choices made have led to the results obtained and whether or not they are appropriate. This includes incorporating considerations for uncertainty, in particular when results are applied for decision making.

* Context-dependent assessment methods should be developed through stakeholder communities, including those who have first-hand experiences of the problem. To complete assessments, databases of metrics are required, alongside guidance on how criticality assessments should be conducted, for which a framework for criticality assessments has been provided.

- It is necessary to build on metric-based assessment methods by developing scenarios of future material supply and demand.

Implementing these recommendations will not be easy, although the problem of materials criticality deserves significantly more attention if businesses and the economy are to become more sustainable in the long term.

\section{Acknowledgements}

This paper was completed as part of an Engineering Doctorate project at the University of Surrey Centre for Environmental Strategy, co-sponsored by Rolls-Royce with support from the Samulet (Strategic Affordable Manufacturing in the UK through Leading Environmental Technologies) programme. Financial support from the Engineering and Physical Science
Research Council and the Technology Strategy Board is acknowledged.

Conversations with the following people (in alphabetical order) have contributed significantly to the authors' views on materials criticality: Conny Bakker, Andrew Bloodworth, Roland Clift, James Goddin, Thomas Graedel, Andrew 'Gus' Gunn, Paul Lusty, Kim Marshall, David Peck, Jim Petrie and Simon Ryder.

\section{REFERENCES}

AEA Technology (2010) Review of the Future Resource Risks Faced by UK Businesses and an Assessment of Future Viability. Department for Environment, Food and Rural Affairs (Defra), London, UK.

Alonso E, Gregory J, Field F and Kirchain R (2007) Material availability and the supply chain: Risks, effects and responses. Environmental Science and Technology 41(19): 6649-6656.

Alonso $E$, Sherman AM, Wallington T et al. (2012) Evaluating rare earth element availability: A case with revolutionary demand from clean technologies. Environmental Science and Technology 46(6): 3406-3414.

BGS (2011) Risk List 2011. BGS, Keyworth, UK, see www. bgs.ac.uk/mineralsuk/statistics/riskList.html (accessed 14/09/2011).

Cohen D (2007) Earth audit. New Scientist 194(2605): 34-41.

Duclos SJ, Otto JP and Konitzer DG (2010) Design in an era of constrained resources. Mechanical Engineering 132(9): 36-40.

Elghali L, Clift R, Begg KG and McLaren S (2008) Decision support methodology for complex contexts. Proceedings of the Institution of Civil Engineers - Engineering Sustainability 161(1): 7-22.

Erdmann $L$ and Graedel TE (2011) Criticality of non-fuel minerals: A review of major approaches and analyses. Environmental Science and Technology 45(18): 7620-7630.

European Commission (2010) Critical Raw Materials for the $E U$. European Commission, Brussels, Belgium.

Gordon RB, Bertram M and Graedel TE (2007) On the sustainability of metal supplies: A response to Tilton and Lagos. Resources Policy 32(1-2): 24-28.

Graedel TE, Allwood J, Birat JP et al. (2011) What do we know about metal recycling rates? Journal of Industrial Ecology 15(3): 355-366.

Graedel TE, Barr RM, Chandler C et al. (2012) Methodology of metal criticality determination. Environmental Science and Technology 46(2): 1063-1070.

Gruber PW, Medina PA, Keoleian GA et al. (2011) Global lithium availability: A constraint for electric vehicles? Journal of Industrial Ecology 15(5): 760-775.

Kaufmann D, Kraay A and Mastruzzi M (2010) The Worldwide Governance Indicators. World Bank, Washington, D.C., USA, Policy Research Paper 5430. 
Kleijn R and van der Voet E (2010) Resource constraints in a hydrogen economy based on renewable energy sources: An exploration. Renewable and Sustainable Energy Reviews 14(9): 2784-2795.

Lee $\mathrm{CH}$ (1998) Formulation of resource depletion index. Resources, Conservation and Recycling 24(3-4): 285-298.

Lempert R, Hallsworth M, Hoorens S and Ling T (2009) Looking Back on Looking Forwards: A Review of Evaluative Scenario Literature. European Environment Agency, Copenhagen, Denmark.

Lloyd S, Lee J, Clifton A, Elghali L and France C (2011) The likelihood of restrictions in the availability of metallic resources. Proceedings of the 2011 Engineering Doctorate Conference, University of Surrey, Guildford, UK, pp. 283-297.

Messner JJ (ed.) (2011) Failed States Index 2011. The Fund for Peace, Washington, DC, USA.

Morley N and Eatherley D (2008) Materials Security: Ensuring Resource Availability for the UK economy. C-Tech Innovation Ltd, Chester, UK.

Nassar N, Barr R, Browning M et al. (2012) Criticality of the geological copper family. Environmental Science and Technology 46(2): 1071-1078.

National Research Council (2008) Minerals, Critical Minerals and the U.S. Economy. National Academies Press, Washington, DC, USA.
Neumayer E (2000) Scarce or abundant? The economics of natural resource availability. Journal of Economic Surveys 14(3): 307-335.

Rosenau-Tornow D, Buchholz P, Riemann A and Wagner M (2009) Assessing the long-term supply risks for mineral raw materials - a combined evaluation of past and future trends. Resources Policy 34(4): 161-175.

Sohn I (2006) Long term projections of non-fuel minerals: we were wrong, but why? Resources Policy 30(4): 259-284.

Steen BA (2006) Abiotic resource depletion: Different perceptions of the problem with mineral deposits. International Journal of Life Cycle Assessment 11(1): 49-54.

Taleb NN, Goldstein DG and Spitznagel MW (2009) The six mistakes executives make in risk management. Harvard Business Review 87(10): 78-81.

Tilton JE (1996) Exhaustible resources and sustainable development. Resources Policy 22(1/2): 91-97.

Tilton JE (2003) On Borrowed Time? Assessing the Threat of Mineral Depletion. Resources for the Future, Washington, DC, USA.

Tilton JE and Lagos $G$ (2007) Assessing the long-run availability of copper. Resources Policy 32(1-2): 19-23.

Yang CJ (2009) An impending platinum crisis and its implications for the future of the automobile. Energy Policy 37(5): 1805-1808.

\section{WHAT DO YOU THINK?}

To discuss this paper, please email up to 500 words to the editor at journals@ice.org.uk. Your contribution will be forwarded to the author(s) for a reply and, if considered appropriate by the editorial panel, will be published as a discussion in a future issue of the journal.

Proceedings journals rely entirely on contributions sent in by civil engineering professionals, academics and students. Papers should be 2000-5000 words long (briefing papers should be 1000-2000 words long), with adequate illustrations and references. You can submit your paper online via www.icevirtuallibrary.com/content/journals, where you will also find detailed author guidelines. 


\section{A framework for environmental risk management}

S. Lloyd stafford.lloyd@rolls-royce.com

A. Clifton

Rolls-Royce

Bristol, UK

J. Lee, L. Elghali and C. France

Centre for Environmental Strategy

University of Surrey

Guildford, UK

\section{ABSTRACT}

Aero engine designs can have a life time of over 45 years, which is long enough for the understanding of environmental problems to change significantly. This places the aero engine designer in a position of uncertainty, as unforeseen environmental problems could affect the viability of a design. 'Risk' is used to describe future uncertainties that can lead to undesirable consequences. This paper presents a framework for environmental risk management that allows the designer to answer the question: what is the risk to a design from its environmental impacts over the life cycle? The framework provides a process for turning complex environmental business hazards into a form that can be used to develop mitigating actions within the design process. The paper demonstrates the framework through two examples and discusses findings, leading to conclusions on what is required to implement the framework into a business. 


\section{NOMENCLATURE}

Business risk multiplying the likelihood of a hazard by its potential impact on business objectives

Environmental stakeholder response to environmental impacts with the potential to cause

Business hazard harm to business objectives

Environmental subset of business risks from environmental business hazards

business risk

HHI Herfindahl-Hirschmann Index

LCA Life Cycle Assessment

LCI Life Cycle Inventory

LCIA Life Cycle Impact Assessment

Life cycle Consecutive and interlinked stages of a product system, from raw material extraction, or generation from natural resources, to final disposal ${ }^{(1)}$

NGO Non-Governmental Organisation

Risk management Activities to control and manage risk in an organisation ${ }^{(2)}$

$\mathrm{REACH} \quad$ Registration Evaluation Authorisation and restriction of CHemicals

SVHC Substance of Very High Concern

WGI World Governance Indicator

\subsection{INTRODUCTION}

A constantly evolving awareness of environmental concerns presents a particular problem for designers of aero engines that can have a life time of over 45 years $^{(3)}$. In the latter part of the 20 th century awareness of environmental concerns such as ozone depletion, acid rain and global warming increased to the extent that legislation and social pressures affected businesses, through for example the Montreal protocol on ozone depleting substances ${ }^{(3)}$. To address these issues typical questions for the environmental designer include: what are the environmental impacts over the product life cycle? Which need to be addressed, why and how ${ }^{(4)}$ Answers to these questions are unlikely to be the same now as they will be in 40 years, which leaves the aero engine designer in a position of uncertainty, not knowing whether or not the viability of a design will be affected by an unforeseen environmental problem.

'Risk' lacks universal definition, however it is always used to describe future uncertainties and usually where they can lead to undesirable consequences ${ }^{(5)}$, typically assessed as a two-dimensional calculation of likelihood (related to the uncertainties) and impact (to consequences) ${ }^{(6)}$. Therefore the question for the aero engine designer becomes: what are the risks to a design from its environmental impacts over the life cycle? This paper presents a framework for environmental risk management that produces answers to this question, identifying future environmental business hazards and translating them to be compatible within standard design processes. Although developed for the Rolls-Royce design system, the framework is applicable throughout the aerospace industry and others that produce long life products.

The risk based approach has several benefits. Significantly it does not create a new set of processes for addressing environmental problems in design, instead integrating them into existing design risk management processes in a format that the business can understand. It solves the problem of deciding which environmental impacts need to be addressed, as logically those that pose the 
highest risk will be prioritised. Using risk also provides traction in addressing environmental impacts over the product life cycle, where the 'in use phase' traditionally dominates ${ }^{(3)}$.

This paper provides: (1) a precise definition of what is meant by risk in this context, the specific questions the framework has been designed to answer and why, (2) a description of the framework, and (3) two examples to describe the practical application of the framework. The two examples show that the framework for environmental risk management works, however, whilst the framework process is sound, it does highlight several difficult problems. The paper draws on experiences from working through these problems to conclude with recommendations for implementing the framework into a business.

\subsection{PROBLEM DEFINITION}

Business risk is a well established concept (details can be found in the relevant British standards ${ }^{(2,7)}$ ). It is concerned with identifying hazards that have the potential to impact on business objectives. Risk is defined here as the product of the likelihood of a hazard occurring with the impact it would have on the business if it $\operatorname{did}^{(6)}$.

There are many sources of harm that can impact on business objectives. This paper is concerned with one type in particular. Termed 'environmental business risks', they have been defined from the observation that if a business has an impact on the environment, this in turn can create circumstances that will lead to an impact on business objectives. As observed by Matten ${ }^{(8)}$ and Sharratt and Choong ${ }^{(9)}$, these circumstances are manifested not directly from the environment itself, but through a stakeholder ${ }^{\dagger}$ response to an environmental impact. Stakeholder responses could come from, for example, regulators (national and international), shareholders, employees, customers, local communities and Non-Governmental Organisations (NGOs) ${ }^{(8,9)}$. Consistent with the definition of environmental business risks, an 'environmental business hazard' is defined here as a stakeholder response to environmental impacts with the potential to impact on business objectives.

Environmental business risks, as defined, are particularly nebulous and difficult to manage. The stakeholder responses can come from a complex mixture of actual, potential or perceived environmental impacts and be influenced by dynamic political factors and societal values. They are not obvious and it is not surprising that businesses tend to overlook them (see e.g. Elkington and Trisoglio, $1996^{(10)}$ ).

The ideal way to mitigate environmental risks is to manage them when a product is being designed, consistent with the pro-active logic behind all environmental design ${ }^{(11)}$. The most successful approaches to environmental design apply a methodology relevant to the decision making context ${ }^{(12,13)}$, which involves using environmental analysis tools to meet the demand for environmental information ${ }^{(14)}$. Risk is consistent with the design decision context at Rolls-Royce. The framework for environmental risk management provides a methodology for integrating environmental risks into existing design risk management processes.

\subsection{Two perspectives}

There are two sides to the problem of managing environmental business risks, as shown in Fig. 1. The design perspective approaches the problem through understanding the environmental impacts of a product, which are the sources of the risk. The question is: how can these environmental impacts lead to an environmental business risk? For example if a design incorporates a substance that is hazardous to the environment, what is the likelihood that the use of this substance will be restricted by regulation leading to an impact on business objectives?

${ }^{\dagger}$ Here stakeholders are broadly defined as anyone who can affect or is affected by the activities of an organisation ${ }^{(9)}$. 


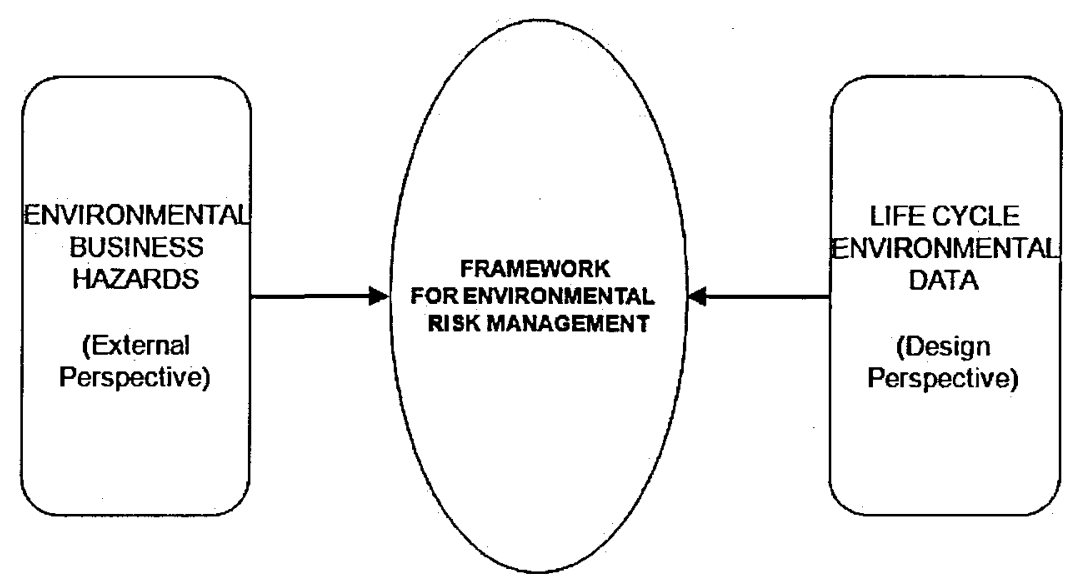

Figure 1. Design and external perspectives on environmental business risk.

The external perspective approaches the problem through attempting to identify environmental business hazards more broadly. The question is: what existing and future positions on environmental issues might occur that would impact on the expected product portfolio? For example, is future regulation likely to restrict the use of environmentally hazardous materials used in our products?

The framework captures the interplay between product environmental impacts and environmental business hazards, feeding connections between the two into standard risk management processes.

From both the design and external perspectives the framework needs to incorporate a means of understanding the environmental impacts of a product. Considering the whole product life cycle, defined as consecutive and interlinked stages of a product system from raw material extraction to final disposal ${ }^{(1)}$ (Fig. 2), is important. Even though significant environmental impacts across the life cycle will not be directly related to an organisation, events from indirect environmental impacts can have an effect on objectives ${ }^{(16)}$. Life Cycle Assessment (LCA) provides a comprehensive way of collating and understanding the environmental impacts of a product, although it is usually simplified as an environmental design tool due to its complexity ${ }^{(17)}$.

The incorporation of a simplified product based environmental assessment is represented in Fig. 1 as life cycle environmental data. The challenge is to link this data to environmental hazards and vice versa. The remainder of the framework that makes this link has been built upon the standard steps within risk management ${ }^{(2)}$, addressing the following questions:

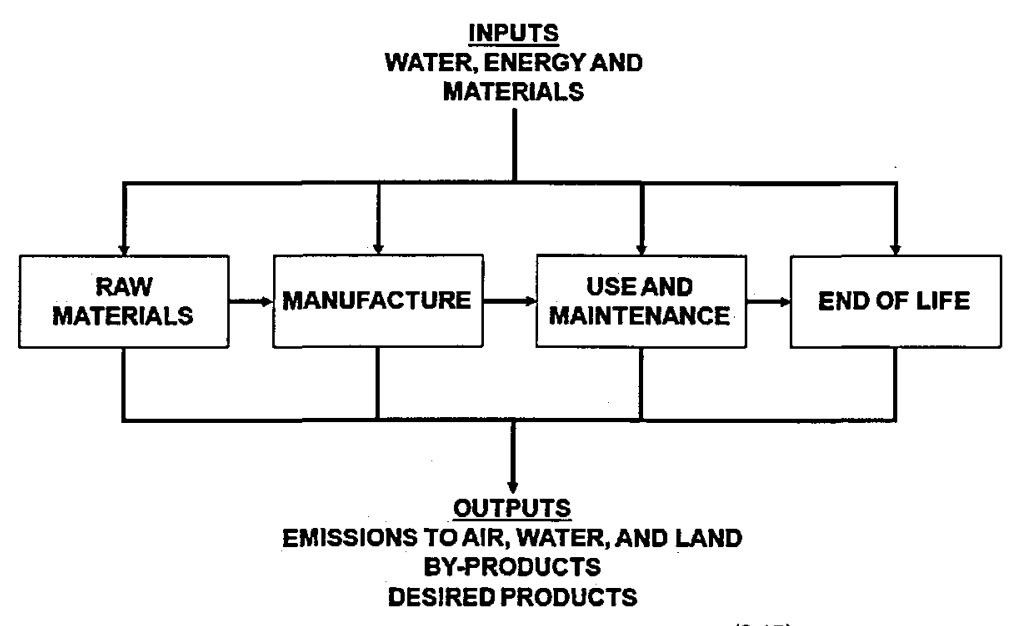

Figure 2. Simplified product life cycle ${ }^{(3,15)}$. 


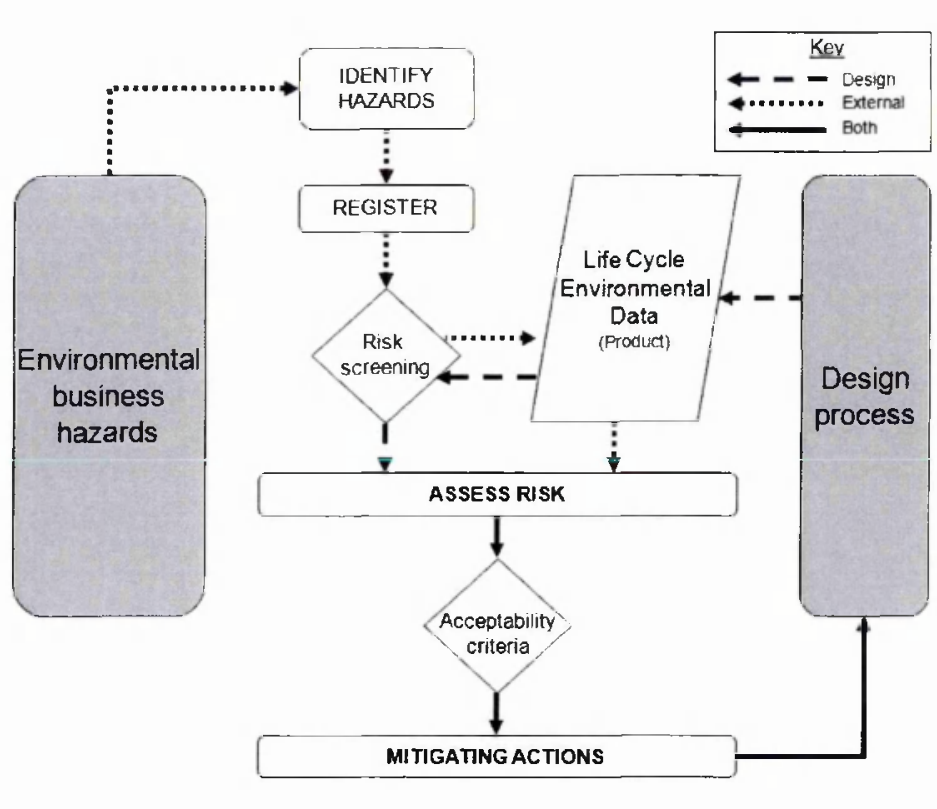

Figure 3. Framework for environmental risk mannagement.

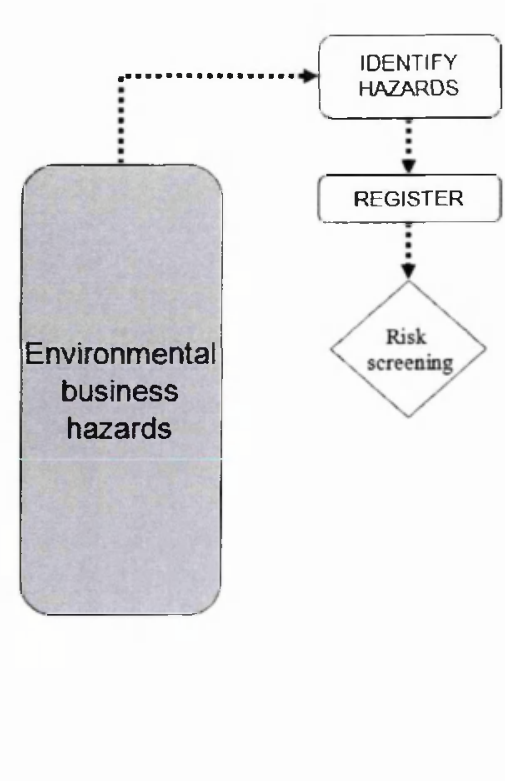

Figure 4. Identifying hazards.

- How are environmental business hazards identified?

- How are likelihood and impact assessed to measure the environmental business risk?

- How are the risks evaluated to understand whether or not mitigating actions are required within the design process?

Businesses already consider risk within design processes. The framework provides a means of using existing risk management methods to address environmental business risks in the same way.

\subsection{FRAMEWORK}

The framework for environmental risk management is presented in Fig. 3, connecting product life cycle environmental data from the right hand side of the figure with environmental business hazards on the left. To describe the framework it is separated into three parts:

1. Identifying environmental business hazards.

2. Connecting hazards and products, from both the external and design perspectives.

3. Assessing risk and defining mitigating actions.

\subsection{Identifying environmental business hazards}

For ease of explanation, the process for identifying hazards is highlighted from the rest of the framework in Fig. 4. It consists of two steps:

1. identifying environmental business hazards

2. risk screening.

There are numerous environmental business hazards, occurring over the product life cycle. As mentioned before they are not necessarily obvious. Identifying them is a complex task, requiring judgements from people with sufficient knowledge of environmental problems and how these are 


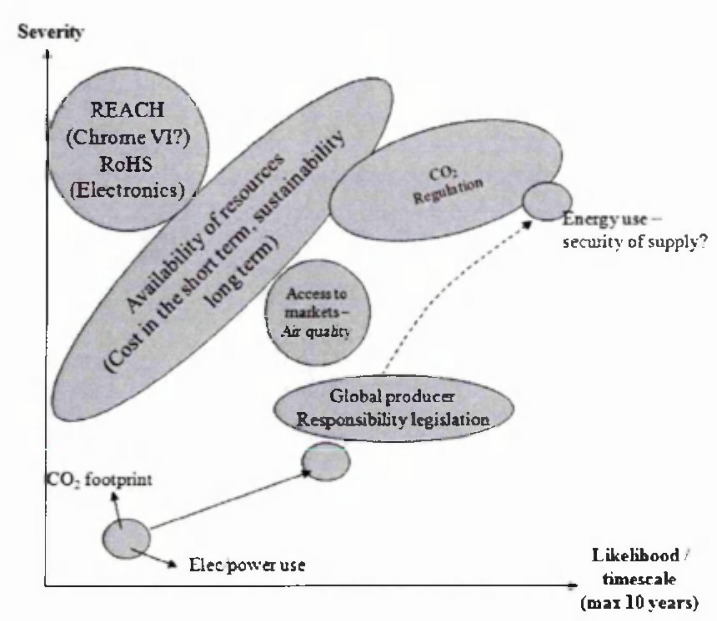

Figure 5. Example risk screening chart.

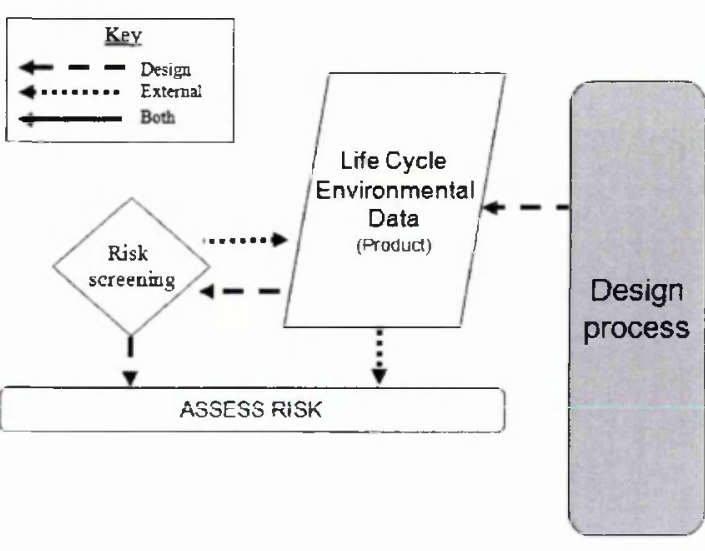

Figure 6. Connecting hazards and products.

relevant to a business' products. The subjective nature of identifying hazards is prevalent within many contexts of risk management. In this case, hazards were identified through the use of focus groups to capture the judgements of environmental specialists within Rolls-Royce. Other social research methods could have been used, such as interviews or more informal workshops. Focus groups were chosen to facilitate discussion.

There are likely to be a number of environmental hazards identified; risk screening is requircd to prioritise those that require a quantitative risk assessment. These prioritised hazards have an identifiable impact on the business that is of sufficient concern to warrant a quantitative assessment. To screen hazards judgements are required from people with knowledge of the hazard (for example, whether it relates to specific emissions, substances or materials) but also of the business, its objectives, its products and how the hazard can impact upon these. Judgements are also required to assess whether or not the hazards are of sufficient concern, which is dependant on the veracity of the hazard and how it is perceived by the business.

In effect, risk screening is a risk assessment, although it relies upon judgements of impact and likelihood rather than a quantificd assessment. Since the framework was developed primarily for use within Rolls-Royce, focus groups of key personnel within the business were used to screen hazards, capturing judgements of likelihood (or the estimated timescale over which the hazard might occur) and significance using a simple chart as shown in Fig. 5. Hazards towards the top of the chart were prioritised for quantitative risk assessment.

\subsection{Connecting hazards and products}

The next part of the framework connects hazards with products, highlighted for ease of explanation in Fig. 6. A business' objectives are set around the provision of products. By connecting a hazard to a product it can be understood how the hazard affects the ability of the business to provide that product. The impact this would have on business objectives if it did can then be quantified.

By definition, hazards come from environmental impacts, whether or not they are directly or indirectly related to the organisation, across the product life cycle. Life cycle environmental data provides an assessment of the environmental impacts of a product. What is required is to connect the two together. How the connection is made depends on whether or not it is done from the design or external perspective. 


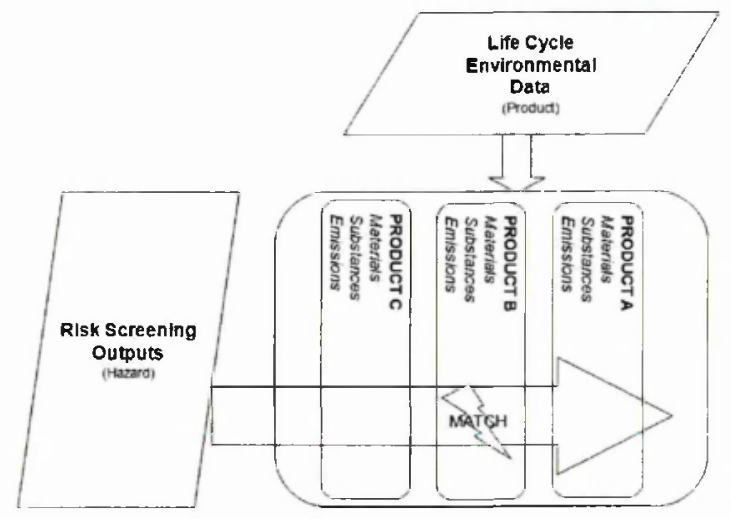

Figure 7. Connecting hazards and products from the external perspective.

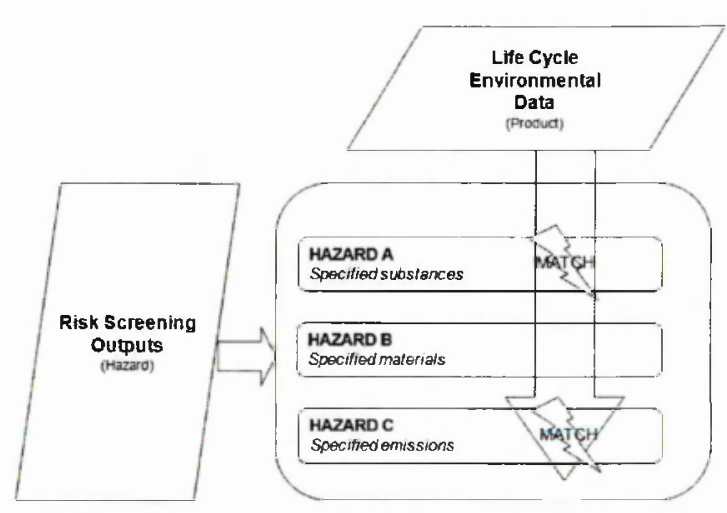

Figure 8 . Connecting a product with hazards from the design perspective.

Making the connection from the external perspective is shown in Fig. 7. Hazards are connected to products by matching the known emission, substance or material that is the source of the hazard with product life cycle environmental data. A quantified risk assessment can then be completed.

The design perspective works the other way around, as shown in Fig. 8. A set of product life cycle environment data, containing emissions, substances and matcrials, is matched with hazards that have come out of the risk screening process. A quantified risk assessment can be completed when a connection is made between an aspect of the product life cycle environmental data and one related to a hazard.

\subsection{Assessing risk and mitigating actions}

The final steps of the framework are highlighted in Fig. 9, providing a process for quantifying the risk from prioritised hazards and evaluating it to define mitigating actions. Risk is quantified by assessing the likelihood that an environmental hazard will occur and multiplying it by the business impact it would have if it did. Information on significant risks can then be fed back to the design processes and appropriate modifications applied. Assessing risk is the same irrespective of whether the starting point is the external or design perspective.

Environmental business risks should be managed like any other risks within an organisation's standard risk management systems, which provide:

- The objectives of the business, required to determine the impact of the risk.

- A common methodology for combining the likelihood and impact to quantify risk.

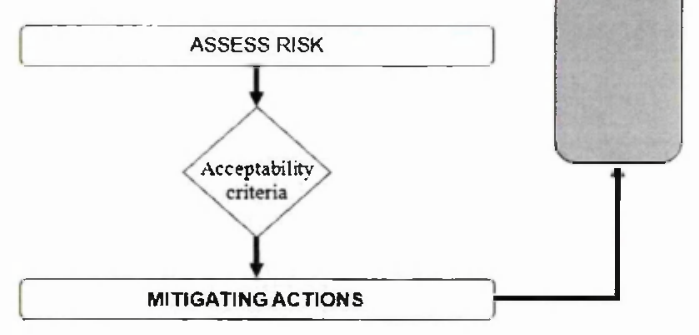

Figure 9. Assessing risk and mitigating actions.
Risk criteria for evaluating the significance of the risk and whether mitigating actions are required.

- Risk management processes enabling the integration of environmental business risks into existing design and management systems. 


\begin{tabular}{|c|c|c|c|c|c|c|}
\hline & $\begin{array}{c}\text { Very high } \\
>50 \%\end{array}$ & 5 & 10 & 15 & 20 & 25 \\
\cline { 2 - 7 } & $\begin{array}{c}\text { High } \\
25 \%<\mathrm{L} \leq 50 \%\end{array}$ & 4 & 8 & 12 & 16 & 20 \\
\cline { 2 - 7 } & $\begin{array}{c}\text { Medium } \\
5 \%<\mathrm{L} \leq 25 \%\end{array}$ & 3 & 6 & 9 & 12 & 15 \\
\cline { 2 - 7 } & $\begin{array}{c}\text { Low } \\
1 \%<\mathrm{L} \leq 5 \%\end{array}$ & 2 & 4 & 6 & 8 & 10 \\
\hdashline $\begin{array}{c}\text { Very low } \\
\leq 1 \% \%\end{array}$ & 1 & 2 & 3 & 4 & 5 \\
\hline
\end{tabular}

Figure 10. Sample impact likelihood matrix.

\subsubsection{Risk assessment}

The standard technique for risk assessments used within Rolls-Royce is 'if...then' statements. Qualifying and multiplying the likelihood of the 'if' and the impact of the 'then' provides structure to the risk assessment. For example:

If emission $X$ is regulated to certain levels... Then the cost of the process will double to keep emission $X$ within limits;

If the cost of the process doubles... Then this will increase the unit cost of parts that are manufactured using the process;'

If the cost of the part increases... Then product margins will be reduced decreasing profits.

In this example, the risk can be quantified by assessing the likelihood of emission $\mathrm{X}$ being regulated and calculating the potential decrease in profit if it was.

Assessing risk requires a common methodology to measure likelihood, impact and multiply them together, so risks from different sources can be compared and prioritised ${ }^{(5)}$. The Rolls-Royce system applies a risk matrix similar to the one shown in Fig. 10.

In this matrix, likelihood can either be represented on a percentage scale or based on judgements varying from very low to very high. Impact is measured in $\mathfrak{E}$ s. The scale of impact varies depending on the level of the business at which the risk assessment is being performed. A very high impact at a project level will be viewed as lower when assessed at the level of the whole business. Each measure of likelihood and impact is given a score of between one and five, which are then multiplied together to quantify the risk.

As the example demonstrates, the likelihood of an environmental risk will depend on the hazard. In the example given above this would be the likelihood that an emission with a known environmental impact will be subject to regulation. It may be that an assessment of the likelihood is dependent on expert judgements, for example from an cnvironmental manager with knowledge of emissions regulations. It may also be the case that a more deterministic approach can be used. Either way the assessment of likelihood needs to be done in a meaningful way, represented on the scale applied within the risk matrix.

The business impact is related to the business' objectives, which are based on the provision of products, related services and the profit that this provides. For cxample the profit for providing aero engines to airlines, calculated by subtracting the cost of providing the engine from revenue. 
An environmental business risk is likely to impact on the ability of the business to provide these products by either:

1. Affecting the ability of the business to manufacture a product, for example through restrictions on the availability of substances or materials.

2. Affecting product cost.

The impact will be a function of how the hazard affects the product. In the example above the impact on profits may be quite small, as the hazard only increases the cost of one process used to manufacture a product. However the risk will be large if it places a restriction on the ability of the business to make a product, for example if the use of certain substances is banned. All revenue related to the provision of that product is potentially affected. The impact is also likely to be large if it affects more than one product.

\subsubsection{Mitigating actions and residual risks}

The final step in the process evaluates the risk to determine if mitigating actions are required. Risks are evaluated against risk criteria, which outline thresholds for the acceptability of risks, above which mitigating actions will be required. Risk criteria will typically be defined within an organisation's risk management system.

At Rolls-Royce the acceptability of risks is defined at the various levels of the organisation at which risks are assessed, from across the whole group down to an individual project, as an initial input to the risk management process. Whilst the level at which risks become unacceptable is not prescribed, typically the following levels of risk require mitigating actions:

- any risk with a very high potential impact;

- high impact and a likelihood of medium ( $>5 \%)$ or higher; or

- medium impact and very high likelihood (>50\%).

This is displayed by the shaded area towards the right hand side of the risk matrix in Fig. 10.

If a risk is beyond acceptability criteria, the process examines how they could be mitigated. Possible actions include:

- Replacing the source of the environmental risk, for example a harmful substance or scarce material.

- Developing alternatives if they are not available.

- Re-design components or processes to remove the source of the environmental risk.

The process also provides a means of identifying and assessing residual risks associated with mitigating actions, for example the costs of developing alternatives, achieving regulatory compliance or re-designing a component. There may also be residual risks associated with increases in product cost, should a re-designed part be more expensive, or if the component life is reduced affecting maintenance costs.

\subsection{EXAMPLES}

The following sections use two environmental hazards to describe how the framework for environmental risk management works in practice, from the design and external perspective. These hazards were prioritised as an output of the risk screening example shown in Fig. 5. 


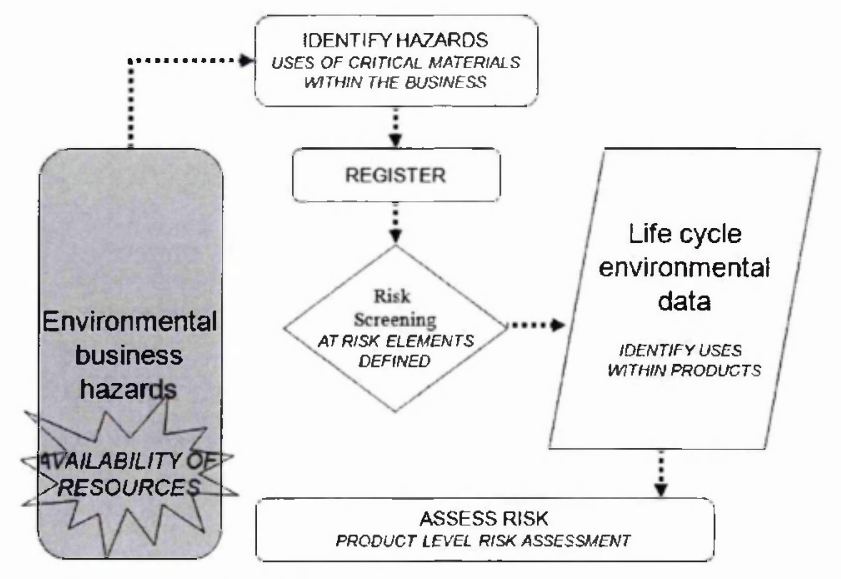

Figure 11. Availability of resources from the external perspective.

1. External: Availability of resources, using the example of platinum (Pt), which is used in coatings for turbine blades.

2. Design: Registration, Evaluation, Authorisation and restriction of Chemicals (REACH), a European regulation related to the use of chemicals, using the example Trichloroethylene.

\subsection{External perspective: Availability of resources}

Availability of resources refers to the phenomenon of constraints being placed on the accessibility of material commodities as a result of geological, environmental, social and cconomic factors. An example case is the Cobalt crisis in the $1970 \mathrm{~s}^{(15)}$. It is not solcly an environmental problem, however accounting for the depletion of resources is incorporated within Life Cycle Impact Assessment $(\mathrm{LCIA})^{\dagger}$ and it falls within the definition of an environmental business risk. Whilst the availability of resources is not a new problem, the increasing dependency of modern industry on scarce elements has recently raised awareness of the risks. The complexity of the issues makes it impossible to predict with certainty when or if a material supply restriction might occur and what the effect might be on availability or price. However, businesses are beginning to develop ways of assessing the risks in order to implement any necessary mitigating actions ${ }^{(19)}$.

From the external perspective of the framework for environmental risk management, it is necessary to identify materials that have a likelihood of supply restrictions and determine where these are used within products through life cycle environmental data. Analysing historical price volatility or making qualitative judgements of the importance of a material to the business can be used to identify materials that are important to the business and are likely to become unavailable.

Connecting the use of an element, for example platinum, to a product facilitates an assessment of the business risk (Fig. 11).

\subsubsection{Risk assessment}

The risks related to the availability of resources are a function of the:

1. likelihood of a material's supply restriction

2. potential impact on the business should a restriction occur.

Several recent papers and reports have been published on the topic of 'materials scarcity', which have sought to assess the likelihood of materials supply restrictions, for example, European 
Commission $^{(20)}$, DEFRA ${ }^{(21)}$, Duclos et $a l^{(19)}$, Morley and Eatherley ${ }^{(22)}$ and Alonso et $a l^{(15)}$. Each study approaches the topic slightly differently, although they all apply an assessment method using static metrics. The metrics are developed to represent the underlying mechanisms that can place a restriction on the availability of materials. Materials are then assessed using the metrics to determine the likelihood that they will be restricted in the future. Alonso ${ }^{(23)}$ highlights limitations of the static metric approach, which fails to "capture the dynamics of the materials systems they attempt to represent' (p61). Modelling the dynamics of materials systems provides a more accurate assessment, although this has only recently become the subject of active research.

Developing a likelihood assessment method is beyond the scope of this paper, so the method developed for the European Commission ${ }^{(20)}$ has been applied. The EC method has been selected as it presents the most transparent methodology, with publicly available data so the assessment can be repeated. The EC report assessed 'supply risk' (the likelihood of a supply restriction) using the following metrics:

- Herfindahl-Hirshmann Index (HHI): a measure of supply diversity used to highlight monopolies that could increase the likelihood of a supply restriction. The HHI is calculated by summing the squares of the supply percentages $\left(S_{\text {ic }}\right)$ of producing countries $(c)$ for a given material $(i)$, which produces a score of between 0 and 10,000:

$$
H H I_{c}=\sum_{c} S_{i c}^{2}
$$

- World Governance Indicators (WGI): a measure of political stability calculated by the World Bank, on a scale of $-2 \cdot 5$ to $2 \cdot 5$, which was linearly adjusted to 0 to 10 (10 representing the highest possible risk). Political stability highlights materials that come from unstable producing regions, increasing the likelihood of a material supply restriction. Scaled WGI scores were multiplied by the supply percentages of producing countries to produce a modified HHI, with a possible score of 0 to 100,000 , scaled to 0 to 10 :

$$
H H I_{W G I}=\sum_{c} S_{i c}^{2} W G I_{c}
$$

- Recycling rate $(\rho)$, percentage of supply met from old scrap, based on the assumption that an increased reliance on recycled sources reduces the likelihood of a restriction, presented as a decimal, based on EU data.

- Substitutability $(\sigma)$ : how easily the use of a material can be substituted in all applications within the economy, based on the assumption that restrictions in the supply of a material will only have an economic impact if it cannot be substituted. The following decimals were used, with the assessment performed by material using expert judgement.

Table 1

\section{Substitutability indices ${ }^{(20)}$}

$\begin{array}{cc}\begin{array}{c}\text { Score } \\ 0\end{array} & \begin{array}{c}\text { Substitutability potential } \\ 0.3\end{array} \\ 0 \cdot 7 & \text { Easily and completely substitutable at no additional cost } \\ 1 & \text { Substitutable at low cost } \\ & \text { Not substitutable }\end{array}$


Supply Risk (SR) is calculated for a material (i), by multiplying the HHIWGI scores by the recycling rate $\left(\rho_{i}\right)$ and substitutability index $\left(\sigma_{i}\right)$ as shown below, with a theoretical maximum score of 10 , minimum 0 :

$$
S R_{i}=\sigma_{i}\left(1-\rho_{i}\right) H H I_{W G I}
$$

Results for elements with the five highest SR scores are presented in Table 2.

\section{Table 2}

\section{Top five results for Supply Risk (SR) ${ }^{(20)}$}

$\begin{array}{cc}\text { Material } & \text { SR } \\ \text { Rare Earth Elements (REEs) } & 4 \cdot 9 \\ \text { Platinum Group Metals (PGMs) } & 3 \cdot 6 \\ \text { Niobium } & 2 \cdot 8 \\ \text { Germanium } & 2 \cdot 7 \\ \text { Antimony } & 2 \cdot 6\end{array}$

In reality it is very unlikely that a material will score 10 in the SR assessment, as this would mean a material is $100 \%$ sourced from a country with a WGI score of 10 (the Democratic Republic of Congo had the highest score of $8 \cdot 6^{(20)}$ ), which is not substitutable or recycled in anyway.

Reflecting the actual results shown in Table 2, with a highest score of 4.9 for rare earth elements, the SR scores have been calibrated to the likelihood scale applied within the Rolls-Royce risk matrix (Fig. 10), giving the assessment method shown in Table 3 . This scale was used to assess the likelihood of a materials supply restriction in this case.

\begin{tabular}{ccc}
\multicolumn{3}{c}{ Table 3 } \\
Likelihood assessment \\
Score & Likelihood $(L)$ & SR score \\
5 & Very high & \\
& $>50 \%$ & SR $\geq 4$ \\
4 & High & \\
& $25 \%<\mathrm{L} \leq 50 \%$ & $3 \leq \mathrm{SR}<4$ \\
3 & Medium & \\
& $5 \%<\mathrm{L} \leq 25 \%$ & $2 \leq \mathrm{SR} \leq 3$ \\
2 & Low & \\
& $1 \%$ & $<\mathrm{L} \leq 5 \%$ \\
1 & Very low & $1 \leq \mathrm{SR}<2$ \\
& $\leq 1 \%$ & $\mathrm{SR}<1$
\end{tabular}

The impact of a supply restriction on a business is a function of where a material is used, what business objectives are related to where it is used, and how the likelihood of a supply restriction will have an impact on these objectives. To assess impact two approaches can be taken:

1. Calculate the business impact based on the revenue effect should a material become unavailable, i.e. all revenue attached to a product reliant on a material is at risk.

2. Estimate levels of effect, on material availability or price, related to the likelihood of a material supply restriction. The impact on business objectives can then be calculated using this level of effect. 
In reality the likelihood of material supply restriction will be reflected in prices, so the second calculation method is preferred.

A level of effect was estimated by analysing the historical price volatility $(P V)$ of materials, dividing the maximum market price by the minimum over a ten year period:

$$
P V_{i}=\text { Max.price } / \text { Min.price }_{10 \text { years }}
$$

The impact on the business was calculated by multiplying the current price of a material by its price volatility and calculating how the increase in cost will affect business revenue.

\subsubsection{Example: Platinum}

Platinum (Pt) has been identified as a potentially critical element, being expensive, geologically scarce and having few sources of supply. Dispersive uses, for example as a catalyst, also make it difficult to recycle, increasing dependence on primary production ${ }^{(20)}$. The SR score for platinum from Table 2 is $3 \cdot 6$, which means it has a high likelihood of a supply restriction using Table 3 . The price volatility of platinum over a ten year period is $5^{\dagger \ddagger}$. To calculate the potential impact on business revenues the price will be assumed to increase by this amount.

Platinum is used within aero engines as a coating for turbine blades. The potential impact on business revenues from the scarcity of platinum can be calculated by estimating the increase in the cost of turbine blades if the price of the coating was to increase by a factor of 5 . Table 4 presents a simple hypothetical unit cost breakdown for a turbine blade and the effect of an increase in the price of the coating.

\section{Table 4 \\ Hypothetical unit cost breakdown for a turbine blade with and without potential cost increase}

$\begin{array}{ccc}\text { Cost element } & \text { Existing cost of Pt } & \text { New cost of Pt } \\ \text { Materials } & £ 150 & £ 150 \\ \text { Mould manufacture } & £ 25 & £ 25 \\ \text { Casting process } & £ 75 & £ 75 \\ \text { Machining } & £ 100 & £ 100 \\ \text { Finishing ops } & £ 25 & £ 25 \\ \text { Coating } & £ 100 & £ 500 \\ \text { Finishing ops } & £ 25 & £ 25 \\ \text { Total } & £ 500 & £ 900\end{array}$

To estimate the impact on the business it is necessary to determine the effect the cost increase in turbine blades will have on contract revenues. Table 5 presents an aggregated impact on revenues, multiplying the unit cost increase using hypothetical figures of the number of blades in an engine and how many engines make up a commercial contract. It is assumed that there are no service related costs. To estimate the total impact on the business the impact would have to be aggregated across all engines that use a turbine blade with a platinum coating.

Table 5 Aggregating unit cost impact on contract revenues

$\begin{array}{cc}\text { Increase in coated blade unit cost } & £ 400 \\ \text { Coated turbine blades in an engine } & 120 \\ \text { Contracted engines } & 200 \\ \text { Total impact on contract revenues } & £ 9,600,000\end{array}$

${ }^{\dagger \ddagger}$ Rolls-Royce data based on market prices. 


\begin{tabular}{|c|c|c|c|c|c|c|}
\hline \multirow{5}{*}{ 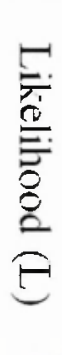 } & $\begin{array}{l}\text { Very high } \\
>50 \%\end{array}$ & 5 & 10 & 15 & 20 & 25 \\
\hline & $\begin{array}{c}\text { High } \\
25 \%<\mathrm{L} \leq 50 \%\end{array}$ & 4 & 8 & 12 & 16 & 20 \\
\hline & $\begin{array}{c}\text { Medium } \\
5 \%<\mathrm{L} \leq 25 \%\end{array}$ & 3 & 6 & 9 & 12 & 15 \\
\hline & $\begin{array}{l}\text { Low } \\
1 \%<\mathrm{L} \leq 5 \%\end{array}$ & 2 & 4 & 6 & 8 & 10 \\
\hline & $\begin{array}{l}\text { very low } \\
\leq 1 \%\end{array}$ & 1 & 2 & 3 & 4 & 5 \\
\hline & & $\begin{array}{l}\text { Very low } \\
\leq f 1 \mathrm{~m}\end{array}$ & $\begin{array}{c}\text { Low } \\
\operatorname{lm}<\ell \leq 5 \mathrm{~m}\end{array}$ & $\begin{array}{c}\text { Medium } \\
5 \mathrm{~m}<\varepsilon \leq 15 \mathrm{~m}\end{array}$ & $\begin{array}{c}\text { High } \\
15 \mathrm{~m}<f \leq 25 \mathrm{~m}\end{array}$ & $\begin{array}{l}\text { Very high } \\
>\neq 25 \mathrm{~m}\end{array}$ \\
\hline & & & & Impac & & \\
\hline
\end{tabular}

Figure 12. Hypothetical risk assessment for platinum.

Figure 12 presents an example risk assessment result, combining the likelihood assessment with the hypothetical impact figures. Such a large increase in cost aggregates to a significant impact on business revenues, reflected within the score on the impact scale, producing a medium risk when multiplied with the high likelihood. Being on the border of the unacceptable region, a risk of this magnitude will require some mitigating actions.

\subsubsection{Availability of resources mitigating actions and residual risks}

There are several mitigating actions that can be taken to reduce the risks associated with the use of platinum:

1. Use a different coating material, or design the blade so a coating is not required.

2. Develop alternative coatings or materials if none are available.

3. Ensure end of life materials recovery to retain platinum within a closed-loop supply chain. (Increasing reliance on recycled sources decreases the likelihood of a supply restriction $\left.{ }^{(2)}\right)$.

4. Use financial instruments to mitigate price risks in the short term.

It is likely that one of more of these options will be pursued to mitigate the risks from the use of platinum. In the short term it would be sensible to manage price volatility financially, for example through agreeing futures contracts. Recycling rates can be improved by developing a closed loop supply chain for material recovery, although design changes may be required to use the coating in a manner that maximises end of life recovery. The most sustainable option might be to remove the reliance on platinum either by re-designing components or developing alternatives. Although this would have to be balanced against the performance benefits of using platinum and the time and money taken to make any changes, including the costs of recertification.

Each of the mitigating actions will carry residual risks. For example, there may be quality concerns over using recycled materials in new components. Re-designing components to encourage end of life recovery or to reduce/remove reliance on platinum will carry costs and may have other detrimental effects on the design. Developing alternatives has residual risks in the costs incurred, together with any other unintended consequences of making design changes. 


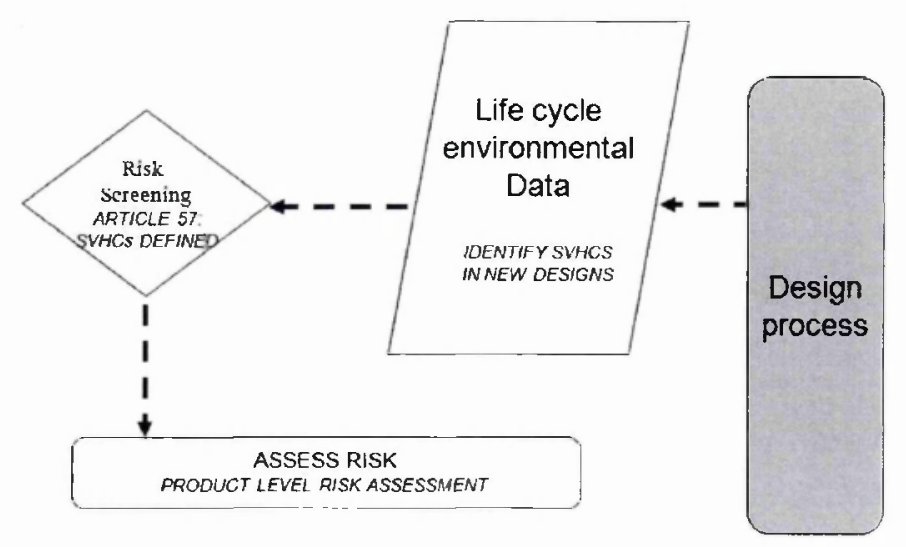

Figure 13. REACH design perspective.

\subsubsection{Benefits}

There are several benefits to managing availability of resources risks in this way:

- Rolls-Royce is aware of the supply risks associated with some of the materials it uses. However, at the moment there is no structured methodology for assessing the risks of a materials supply restriction. The framework for environmental risk management provides a structured process for doing this, allowing the risk to be properly quantified.

- By fully understanding the risks, it is possible for the business to implement an appropriate response through mitigating actions.

- By using the risk management system, risks are integrated into standard business decision making, relevant to the parts of the business that own the risk (the designers of the product), so that something can be done proactively if needed.

- The risk based approach provides a business case for recycling materials and developing end of life plans for components.

\subsection{Design perspective: REACH}

REACH is a European regulation that aims to improve the protection of human health and the environment from the risks ${ }^{\text {th }}$ posed by chemicals ${ }^{(24)}$. The authorisation requirement under REACH seeks to control the risks from particularly harmful substances, categorised as Substances of Very High Concern (SVHCs), through a process of progressive replacement. In time, using a SVHC or placing it on the market will only be allowed if the regulator grants an authorisation (substances requiring authorisation are placed on Annex XIV of the regulation), which is dependant on certain criteria. Any business using SVIICs will be affected, as at some point in the future continued use cannot be guaranteed. It would be prudent for a business to identify its usage of SVHCs and consider what the consequences are if a substance is no longer available.

From the design perspective it is required to identify whether or not any existing or new designs are reliant on the use of SVHCs within a product's life cycle. Life cycle environmental data can be cross-referenced with known SVHCs to begin the risk assessment (Fig. 13). The product level risk assessment is dependant on combining the likelihood of a substance becoming unavailable with the impact this would have on business revenues. Mitigating actions can then be defined within the design process.

\footnotetext{
${ }^{\#+}$ Distinct from business risk, REACH identifies chemicals as the source of undesirable consequences, which impact on human health and the environment.
} 


\subsubsection{REACH risk assessment}

The risk from REACH authorisation is a function of:

1. the likelihood of a substance becoming unavailable due to REACH

2. the impact on business objectives should a substance become unavailable.

The likelihood of a substance becoming unavailable due to REACH is reliant on the regulatory process, specific details of which can be found in the legal text of $\mathrm{REACH}^{(25)}$. The criteria for identifying SVHCs and transitioning them towards authorisation are not entirely clear, as no substances have been all the way through the process. Table 6 presents an assessment of likelihood, developed through knowledge of the legal text using a scale from 1 to 5 .

Table 6

Likelihood of a substance becoming unavailable due to REACH

$\begin{array}{cc}\text { Score } & \text { Likelihood (L) } \\ 5 & \text { Very high } \\ & >50 \% \\ 4 & \text { High } \\ & 25 \%<L \leq 50 \% \\ 3 & \text { Medium } \\ & 5 \%<\mathrm{L} \leq 25 \% \\ 2 & \text { Low } \\ & 1 \%<\mathrm{L} \leq 5 \% \\ 1 & \text { Very low } \\ & \leq 1 \%\end{array}$

REACH requirement

Authorisation required

Substance transferred to Annex XIV, sunset date for authorisation set

Substance on candidate list for transition to Annex XIV

Known SVHC properties, similar substances on candidate list

Known or suspected SVHC properties

In contrast to the previous example, calculating the impact if a substance becomes unavailable due to REACH is straightforward: if a substance is used in the product itself, or in a process to manufacture a product, if the substance is unavailable, the product cannot be made. All revenue

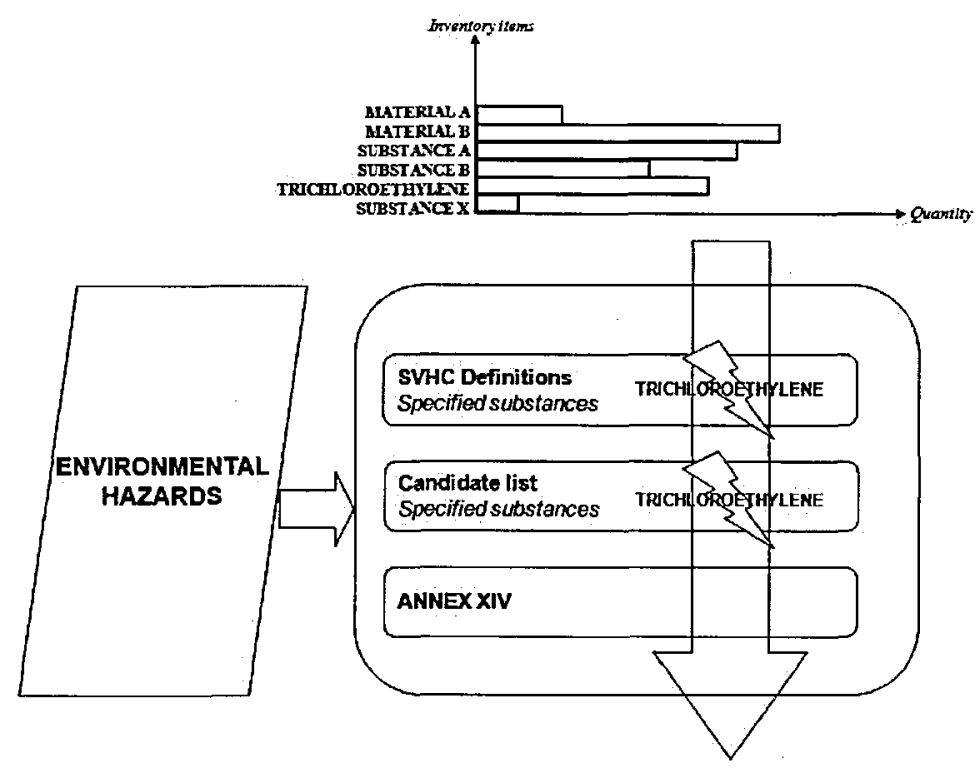

Figure 14. Connecting a product with Trichloroethylene. 


\begin{tabular}{|c|c|c|c|c|c|c|}
\hline \multirow{5}{*}{ 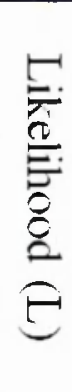 } & $\begin{array}{l}\text { Very high } \\
>50 \%\end{array}$ & 5 & 10 & 15 & 20 & 25 \\
\hline & $\begin{array}{c}\text { High } \\
25 \%<\mathrm{L} \leq 50 \%\end{array}$ & 4 & 8 & 12 & 16 & 20 \\
\hline & $\begin{array}{c}\text { Medium } \\
5 \%<\mathrm{L} \leq 25 \%\end{array}$ & 3 & 6 & 9 & 12 & 15 \\
\hline & $\begin{array}{c}\text { Low } \\
1 \%<L \leq 5 \%\end{array}$ & 2 & 4 & 6 & 8 & 10 \\
\hline & \multirow[t]{3}{*}{$\begin{array}{l}\text { Very low } \\
\leq 1 \%\end{array}$} & 1 & 2 & 3 & 4 & 5 \\
\hline & & $\begin{array}{l}\text { Very low } \\
\leq £ 1 \mathrm{~m}\end{array}$ & $\begin{array}{c}\text { Low } \\
1 \mathrm{~m}<\mathfrak{x} \leq 5 \mathrm{~m}\end{array}$ & $\begin{array}{c}\text { Medium } \\
5 m<£ \leq 15 m\end{array}$ & $\begin{array}{c}\text { High } \\
15 \mathrm{~m}<£ \leq 25 \mathrm{~m}\end{array}$ & $\begin{array}{l}\text { Very high } \\
>£ 25 \mathrm{~m}\end{array}$ \\
\hline & & \multicolumn{5}{|c|}{ Impact (fs) } \\
\hline
\end{tabular}

Figure 15. Risk assessment for Trichloroethylene acid used in corrosion resistant coatings.

attached to the provision of that product will be at risk. The risk assessment can be completed by using the risk matrix (Fig. 10) to combine assessments of the likelihood of a substance becoming unavailable with the impact this will have on business revenue.

\subsubsection{Example: Trichloroethylene}

Trichloroethylene is used in manufacturing processes for degreasing. It is considered to be a carcinogen, mecting one of the SVHC criteria under REACH. Trichloroethylene is currently on the candidate list of substances for transition onto Annex XIV (substance requiring authorisation) ${ }^{(24)}$, which means that the likelihood of it becoming unavailable due to REACH can be given a likelihood score of 3 using Table 6.

To identify if a product uses Trichloroethylene in its life cycle, environmental life cycle data can be cross-referenced with REACH hazard filters as shown in Fig. 14.

If an engine is designed with a process that uses a Trichloroethylene, then if Trichlorocthylene becomes unavailable due to REACH the engine cannot be manufactured. The impact on the business will be significant, as any potential revenues related to the supply of the engine will be at risk, producing a very high $(>£ 25 \mathrm{~m})$ score on the impact scale.

The likelihood and impact assessment are multiplied and the risk assessment presented in Fig. 15. In this case the risk is in the unacceptable region and mitigating actions will be required.

\subsubsection{REACH Mitigating actions and residual risks}

To mitigate the REACH risks associated with Trichloroethylene its use will have to be replaced. Options include:

- use another degreaser

- redesign the process chain so degreasing is not required.

Whatever solution is used, there will be residual risks related to the costs of changing the process. Each re-design option will also carry other residual risks. For example, alternative degreasers may be more expensive increasing process costs. 


\subsubsection{Benefits}

The benefits of managing the risks from REACH in this way are:

- As with the availability of resources case, Rolls-Royce is aware that REACH presents significant business risks. The framework provides a structured process for quantifying them in a way that is consistent with standard risk management processes. Fully understanding the risk allows for measured mitigating actions to be taken.

- One of the main obstacles for understanding the risks related to REACH is making the connection between the use of hazardous substances and products, especially when the substance is used in a process, rather than in the product itself. The need for a product connection highlights the importance of life cycle environmental data within the framework.

- Quantifying and communicating the risks posed by REACH provides traction in removing the use of hazardous substances. In the past, top down policy approaches have been used to communicate substances that cannot be used. Using risk tells engineering teams why substances cannot be used for business reasons and allows them to control risk and its mitigation.

- The framework ensures the designs are checked for substances (either in the product or process) that are likely to be banned at some point in the future. Actions to remove the use of the substance can be taken proactively, making the design more robust.

\subsection{DISCUSSION}

The two examples have shown how the framework for environmental risk management provides a suitable process for managing environmental business risks, turning complex problems into something that the business can comprehend and use to develop mitigating actions within the design process. While the processes outlined in the framework are sound, they do present difficult questions:

- How are environmental business hazards identified?

- How is the likelihood of an environmental business risk assessed?

- What is required to integrate environmental business risks into existing business decision making processes?

- What life cycle environmental data is required?

This paper has shown that the framework works. From the experience of applying the framework, several recommendations can be made on how these questions need to be addressed if the framework were to be implemented, at Rolls-Royce or elsewhere.

As mentioned there will be many environmental business hazards, originating from various stakeholder responses, related to environmental impacts across the product life cycle. In this case focus groups were used to identify hazards, although the groups highlighted difficulties in both how hazards are identified and how a business prioritises hazards for quantified risk assessment. Identifying hazards requires expert knowledge of environmental problems and how a wideranging set of problems can be reduced to a manageable list. Prioritising hazards requires consensus within a business on the relative importance of the issues identified, which will contain subjectivities both within and between organisations.

From experience, the processes of identifying hazards and prioritising them need to be clearly separated. If the process were repeated hazard identification would be done in a more structured 
way, with the use of keywords, for example 'regulation', to prompt judgements from environmental specialists, relevant to each life cycle phase. Eventually, applying a full Life Cycle Impact Assessment (LCIA) to life cycle environmental data could be used as a more deterministic input to hazard identification. Using LCIA would highlight areas of environmental impact within the product system, which may give rise to an environmental business hazard in the future.

Whether or not the hazards matter is a business decision. In this case focus groups were used again, although involving senior managers with input from environmental specialists. In practice, existing risk management systems need to be extended to capture environmental business hazards, involving the relevant business stakeholders who have the responsibility to make the required judgements.

It was proposed that assessing the likelihood of environmental business risks is case specific, which was demonstrated by the two very different approaches used in the examples. In practice, assessing the likelihood of an environmental business risk was very difficult. Likelihood is usually assessed by using statistics to analyse historical data, which in this case does not exist. As a result any assessment will always contain significant uncertainties.

The uncertainty of assessing likelihood raises questions about how environmental business risks will be perceived. Whilst the perception of all risks is subjective, the level of uncertainty in this instance does present a particular problem. More robust assessment methods can be developed, although this will take time. A balance is required between making sure the risk is believable, whilst not creating an analytical burden.

More specific to Rolls-Royce, the experience of completing the examples showed how the framework requires new forms of co-ordination between different parts of the business. Design data was required from engineering, cost data from business groups and contract data from commercial groups, all woven together with the help of risk managers. The framework highlights where processes and responsibilities are required to coordinate different parts of the risk assessment, to ensure effective environmental business risk management.

Another problem to address is defining the requirement for environmental data within the framework, and how this can be supplied with the appropriate environmental assessment tools. The examples have demonstrated that product based life cycle environmental assessment is needed. A simplified form of LCA is the obvious solution. As with setting the goal and scope within LCA, there is still the problem of deciding which environmental impacts are of interest and what data needs to be collected to measure them. Deciding what data to collect can present a circular argument: knowing the hazards defines the data requirement, although to a certain extent data is needed to understand the environmental impacts that lead to the hazards. Rolls-Royce has defined its data requirements through using identified hazards together with data taken from environmental management systems. Over time the data collected will expand as more hazards and requirements are identified.

The data collected for this study was collected as a pilot project. To put the framework into practice a database of manufacturing process and materials production models is being developed, which hold environmental data by process or material. Life cycle environmental data is produced by linking together the materials and process database models for those processes and materials used to make a product. Rolls-Royce has been collaboratively developing a design tool for producing the assessment, which will eventually be integrated into computer aided design systems to remove extra work.

Getting information from supply chains is a common problem when performing product based environmental assessments. Most of the environmental business hazards and environmental management issues affecting Rolls-Royce will affect other businesses in the industry. 
Communicating the associated risks will make the case for data collection. However, common data collection methods are still required so that everyone collects the same data in the same way. RollsRoyce is leading an initiative through cross-industry environmental working groups to develop common data collection methods that can be passed down the supply chain.

\subsection{CONCLUSIONS}

This paper has presented a framework to answer the question: what is the risk to my design from its environmental impacts over the product life cycle? The paper has drawn the following conclusions:

- Environmental business risks are defined as a subset of business risks related to environmental business hazards, which are defined as a stakeholder response to environmental impacts with the potential to cause harm to business objectives.

- A product based life cycle focus is important for managing environmental business risks, connecting external environmental hazards with the source of the risk - product design.

- Two perspectives are defined within the framework for environmental risk management - the external perspective, which captures external events and reflects these within the organisation, and the design perspective, which begins with a set of product based environmental data, cross referencing it with identified hazards.

- The framework provides a means of integrating environmental risks into standard risk management (and by default design) processes - it should not create a new layer of risk management.

- The problem of identifying environmental business hazards needs to be clearly defined in two separate parts, identifying the hazards, and deciding whether or not they matter.

- Assessing the likelihood of environmental risks is complicated, as no historical data exists. A balance is required between developing an assessment method that produces believable results whilst not producing an analytical burden.

- Organisational processes will be required to implement the framework for environmental management, facilitating the required cross-functional working relationships.

- Deciding what data to collect for the framework can create a circular argument. Rolls-Royce used the hazard identification results and data from environmental management as initial requirements for data collection.

- Collecting data from the supply chain would be helped by developing industry standards for environmental data collection.

- The framework requires a tool for creating the required life cycle environmental data in design.

\section{ACKNOWLEDGEMENTS}

This paper was completed as part of an Engineering Doctorate project at the University of Surrey Centre for Environmental Strategy, co-sponsored by Rolls-Royce with support from the SAMULET (Strategic Affordable Manufacturing in the UK with Leading Environmental Technologies) programme. Financial support from the Engineering and Physical Sciences Research Council and the Technology Strategy Board is acknowledged. 


\section{REFERENCES}

1. BSI. BS EN ISO 14040:2006 Environmental management - Life cycle assessment - Principles and framework. British Standards Institution (BSI), London, UK, 2006.

2. BSI. BS ISO 31000:2009 Risk management - Principles and guidelines. British Standards Institution (BSI), London, UK, 2009.

3. LEE, J.J. Greener manufacturing, maintenance and disposal - towards the ACARE targets, Aeronaut $J$, 2006, 110, (1110), pp 567-571.

4. LUTTROPP, C. Ecodesign: What's happening? An overview of the subject area of ecodesign and of the papers in this special issue, J Cleaner Production, 2006, 14, pp 1291-1298.

5. RENN, O. Three decades of risk research: Accomplishments and new challenges. J Risk Research, 1, (1), pp 49-71.

6. RAEng. The Societal Aspects of Risk. Royal Academy of Engineering (RAEng), London, 2003.

7. BSI. BS EN 31010:2010 Risk management - Risk assessment techniques. British Standards Institution (BSI), London, UK, 2010.

8. MATTEN, D. Strategy follows structure: environmental risk management in commercial enterprises, Business Strategy and the Environment, 1995, 4, pp 107-116.

9. Sharratt, P.N. and ChOONG, P.M. A life cycle framework to analyse business risk in process industry projects, J Cleaner Production, 2002, 10, pp 479-493.

10. Elkington, J. and Trisoglio, A. Developing realistic scenarios for the environment: Lessons from Brent Spar, Long Range Planning, 29, (6), pp 762-769.

11. VAN WEENEN, J.C. Towards sustainable product development, J Cleaner Production, 1995, 3, (1-2), pp 95-100.

12. Delay-Saunders, I. Integrating environmental considerations in aircraft design. EngD Thesis, University of Surrey, Guildford, UK, 2006.

13. RYAN, C. Learning from a decade (or so) of ecodesign experience, part 2, $J$ Industrial Ecology, 2006, 8, (4), pp 3-5.

14. Wrisberg, N., Udo de Haes, H.A., Triebswetter, U., Eder, P. and Clift R. (Eds) Analytical Tools for Environmental Design and Management from a Systems Perspective, Kluwer Academic Publishers, 2002.

15. Elghali, L., ClifT, R., BeGG, K.G. and McLaren, S. Decision support methodology for complex contexts, Proceedings of the Institute of Civil Engineers, Engineering Sustainability, 2008, 161, (1), pp 7-22.

16. Alonso, E., Gregory, J., Field, F. and Kirchain, R. Material availability and the supply chain: risks, effects, and responses, Environmental Science and Technology, 2007, 41, (19), pp 6649-6656.

17. BaUmanN, H., Boons, F. and BRAGD, A. Mapping the green product development field: engineering, policy and business perspectives, $J$ Cleaner Production, 2002, 10, pp 409-425.

18. Pennington, D.W., Potting, J., Finnveden, G., Lindeijer, E., Jolliet, O., Rydberg, T. and Rebitzer, G. Life cycle assessment part 2: Current impact assessment practice, Environment International, 2004, 30, pp 721-739.

19. Duclos, S., Otto, J.P. and Konitzer, D.G. Design in an era of constrained resources. Mechanical Engineering, 2010, September, American Society of Mechanical Engineers (ASME).

20. European Commission. Critical raw materials for the EU. European Commission, Brussels, Belgium, 2010.

21. DEFRA. Review of the future resource risks faced by UK business and an assessment of future viability. Department for Environment, Food and Rural Affairs (DEFRA), London, UK, 2010.

22. MORLEY, N. and EATHERLEY, D. Material security: Ensuring resource availability for the UK economy. C-Tech Innovation Ltd., Chester, UK, 2008.

23. Alonso, E. Materials scarcity from the perspective of manufacturing firms: Effects and strategies. PhD Thesis, Massachusetts Institute of Technology, Cambridge, MA, USA, 2010.

24. See www.echa.europa.eu.

25. Commission regulation (EC) no. 1907/2006 of 18 December 2006 concerning the Registration, Evaluation, Authorisation and Restriction of Chemicals (REACH). Official J European Union, L 396/1. 


\title{
Ecodesign through Environmental Risk Management: A Focus on Critical Materials
}

\author{
Stafford Lloyd ${ }^{1,2}$, Jacquetta Lee ${ }^{2}$, Andrew Clifton ${ }^{1}$, Lucia Elghali ${ }^{2}$ and Chris France ${ }^{2}$ \\ ${ }^{1}$ Life Cycle Engineering, Rolls-Royce plc, Bristol, UK \\ ${ }^{2}$ Centre for Environmental Strategy, University of Surrey, Guildford, UK
}

\begin{abstract}
This paper presents an approach to Ecodesign based on the management of environmental business risks, which are defined as 'stakeholder responses to environmental impacts with the potential to cause harm to business objectives'. Case studies are used to demonstrate the approach, with a particular focus on the management of critical materials. The paper concludes that by using risk, environmental considerations can be integrated into design decisions at Rolls-Royce, although the method contains significant uncertainties. In particular, the paper highlights the complexity of both assessing the supply risk of a matcrial and how this could translate into an impact on the business. The paper also discusses how the risk model could be expanded to address other environmental business hazards.
\end{abstract}

Keywords:

critical materials, risk, environmental risk, life cycle management

\section{INTRODUCTION}

Rolls-Royce provides power systems and services for use in the air, on land and at sea, focusing on four main markets: civil aerospace, defence aerospace, energy and marine. Predominantly, although not exclusively, RollsRoyce's products are based on the gas turbine engine.

The nature of Rolls-Royce's products present several unique barriers to the implementation of ecodesign approaches:

1. Environmental impacts from the 'in-use' phase dominate over the product life cycle. Understandably this is the focus for addressing environmental impacts, although this also means that environmental impacts from other phases of the life cycle can be overlooked.

2. Rolls-Royce's products are designed to have an operational life of up to 50 years. Environmental problems can change significantly in this time and it is difficult to foresee what the next problem might be.

3. Rolls-Royce's products are technically mature. There is very little design freedom to make nonuse phase environmental improvements.

4. Due to the safety critical nature of Rolls-Royce's products, the company uses rigorous design systems to verify product designs against well defined requirements. At the present time, nonuse phase environmental impacts are not comparable within the traditional design space, which means they are largely ignored.
Risk management is used within the Rolls-Royce design system to identify hazards that can impact on design and other business objectives. By translating environmental impacts into an assessment of business risk, barriers to ecodesign can be overcome and non use-phase environmental impacts considered within the design process. This paper presents two case studies that test an approach to ecodesign based on the management of environmental risks. The case studies focus on the use of critical materials, which is linked to the abiotic resource depletion environmental impact category.

As risk is a broad term, the paper first defines what is meant by ' 'environmental business risk'. Materials criticality is then introduced as a significant risk, using an approach developed by the European Commission to highlight materials that are of concern. Based on knowledge of where these materials are used in RollsRoyce's products, case study risk assessments are then presented, which show how the risks posed by materials criticality can be incorporated into standard design decisions. The paper discusses the practicalities of the risk based approach, in light of significant uncertainties that will be inherent in any system that seeks to look into the future. The paper also discusses how the risk model could be expanded to address other environmental business hazards.

\section{ENVIRONMENTAL RISK MANAGEMENT}

Environmental risk management adopts a business risk perspective, concerned with identifying hazards that can impact on business objectives [1]. It is based on the 
observation of a cause-effect cycle between the environmental impacts of a business' operations and products and stakcholder responses to these impacts (whether they are actual, potential or perceived) which seek to reduce them and can impact on business objectives [2]. The stakeholder responses to environmental impacts, with the potential to cause harm to business objectives, are defined as 'environmental business hazards'. The environmental business risk is a product of the likelihood of a hazard occurring and the impact it would have on business objectives if it did. There are many sources of environmental business hazard, regulation being a primary example.

Business risk is assessed against objectives, which are generally based upon the provision of products and services and the revenue this provides. It follows that, to assess the risk posed by environmental business hazards, it is necessary to understand how hazards impact on the ability of the business to make and sell products. Risk can be assessed by connecting some feature of the hazard with a feature of the product. Appropriate mitigating actions can then be implemented.

\section{CRITICAL MATERIALS: A SIGNIFICANT ENVIRONMENTAL BUSINESS HAZARD}

A significant environmental business hazard that has been the subject of recent attention is 'critical materials'. The phenomenon is concerned with constraints being placed on the accessibility of material commodities as a result of geological, political and economic factors. Whilst it is not purely an environmental problem, the depletion of abiotic resources is a common impact category within life cycle impact assessment [3], and it falls within the definition of an environmental business hazard.

There are two "dimensions" of materials criticality [4]:

1. Supply risk: identifying and applying factors that can be used to assess the risks to the supply of a material.

2. Economic importance: an assessment of how important the use of a material is in meeting economic goals, which can be also be assessed at a business level e.g. restrictions in the availability of materials can also restrict the ability of a business to make a product.

A material is referred to as critical when it has a high supply risk and is of high cconomic importance.

The two dimensions of materials criticality need to be applied to understand the business risks posed. These can be achieved through the:

1. Identification and application of a method for assessing supply risk, which identifies materials of concern.

2. Connecting these materials with uses in products to evaluate the risk posed to business objectives.

\subsection{Assessing supply risk}

Several different methods have been developed to assess supply risk [5] [6] [7]. Duc to the use of a transparent methodology with available data, this paper focuses on an approach developed by the European Commission (EC) [7]. The EC method applies four metrics to assess supply risk:

1. Monopoly supply: materials that come from few sources are assumed to be higher risk.

2. Governance indicators: materials that arc sourced from politically unstable regions are assumed to be higher risk. Governance indicators are merged with the monopoly supply index to highlight where a material's supply is dominated by unstablc producing countrics.

3. Recycling rate: based on the assumption that the availability of recycled sources lowers risk.

4. Substitutability: materials that are substitutable are likely to be more flexible to changes in demand, reducing risk.

Monopoly supply is measured using the HerfindahlHirschmann Index (HHI), which is the sum of the squares of the supply percentages (S) of producing countries (c) for a given material (i), as shown in Eqn. I:

$$
H H I_{c}=\sum_{c} S_{i c}^{2}
$$

The equation produces a figure between 0 and 10000 , a higher number signifying higher risk.

World Govemance Indicators (WGI) produced by the World Bank were merged with the $\mathrm{HHI}$ to highlight where supply was dominated by an unstable producing region. The WGI scored countries according to 6 categories (including political stability, control of corruption and rule of law), producing a result between 0 and 10 . These scores were merged with the HHI as shown in Eqn. 2:

$$
H H I_{W G I}=\sum_{c} S_{i c}^{2} W G I_{c}
$$

The result produces a score of 0 to 100000 , which was scaled to a value of between 0 and 10 .

The recycling rate used for a material $i\left(\rho_{i}\right)$ applied the ratio of current demand met by old scrap. Data on recycling rate is given in the EC report [7].

Substitutability $\left(\sigma_{i}\right)$ for a material i was measured using an index developed through expert judgements (Table 1).

Table I: Substitutability indices [7]

\begin{tabular}{|l|l|}
\hline Score & \multicolumn{1}{|c|}{ Substitutability } \\
\hline 0 & $\begin{array}{l}\text { Easily and completely substitutable at } \\
\text { no additional cost. }\end{array}$ \\
\hline 0.3 & Substitutable at low cost. \\
\hline 0.7 & $\begin{array}{l}\text { Substitutable at high cost and/or loss of } \\
\text { performance/ }\end{array}$ \\
\hline 1.0 & Not substitutable. \\
\hline
\end{tabular}


All of the metrics were merged into the final equation for supply risk. calculated by material (i):

$$
S R_{i}=\sigma_{i}\left(1-\rho_{i}\right) H H I_{W G I}
$$

The term $\left(1-\rho_{1}\right)$ is used as a higher recycling rate will reduce supply risk. The result of the supply risk calculation will produce a score of between 0 and 10 by material, with a score of 10 representing the highest possible risk.

SR scores were calculated for 41 materials, 14 being highlighted as potentially critical (Table 2 ). Both of the highest scores were attributed to materials that have relatively few sources (monopoly supply).

Table 2: The EC 14 (SR scores in bold) [7]

\begin{tabular}{|c|c|}
\hline Rare Earth Elements (REF) [4.9] & Indium [2.0] \\
\hline Platimum Group Metals (PGM) [3.6] & Tungsten [1.8] \\
\hline Niobium [2.8] & Flunrspar [1.6] \\
\hline Germanium [2.7] & Beryllium [1.4] \\
\hline Antimony [2.6] & Graphitc [1.3] \\
\hline Magnesium [2.6] & Tantalum [1.1] \\
\hline Gallium [2.5] & Cobait [1.1] \\
\hline
\end{tabular}

The 14 critical materials highlighted from the EC report and the method for assessing SR solve the first problem of identifying materials that are of concern. To understand the risk, it necds to be identified where these are used in products.

\subsection{Identifying uses of critical materials}

Product data is required to understand the uses of critical matcrials. Basic data on product content is provided by a standard engineering Bill of Materials ( $\mathrm{BOM}) . \mathrm{A} \mathrm{BoM}$ is unlikely to be sufficient to identify product features related to all environmental business hazards. Hazards can relate to any impact over the product life cycle, requiring a life cycle vicw. However, a BoM is sufficient for identifying uses of critical materials as an initial approach.

There are two perspectives on the problem of identifying critical materials in products. One approach could be to use a list of critical materials (for example the EC 14) and compare these with all product BOMs to identify any matches i.e. where a BoM lists a material of concern. This is necessary to identify all uses of critical materials within cxisting products, and is called the existing product perspective.

The opposite approach would be to start with a BoM and compare it with a list of critical materials to determine if any are used in the product. This is called the design perspective, where only those critical materials in the product being designed are of interest. The next sections focus on assessing the business risks posed by the use of critical materials approaching the problem from both the existing product and design perspectives

\section{CASE STUDY 1: ASSESSING RISKS TO EXISTING PRODUCTS}

The following steps summarise the process for assessing the risks posed by critical materials to an existing product:

1. Identify at risk elements (the EC 14).

2. Identify if those elements are present in a product (required in order to understand the risk).

3. Complete a product based risk assessment by combining the measure of supply risk for the materials in question with the potential impact on business objectives from using the high risk material. To be relevant to existing design processes, the risk assessment has to be completed using the appropriate risk management criteria within Rolls-Royce.

PGMs were identificd as having a high supply risk from the EC report. One particularly rare PGM is used as an alloying element within turbine blades on one of RollsRoyce's large civil aero-engines. Using an alloy with this PGM maintains high creep strength within blades that operate at very high temperatures and pressures [8]

When designing a component, several business objectives are set against which risk can be assessed. One of the main objectives is a target unit cost for the parts and engine. Risks to unit cost of using the PGM in turbine blades can therefore be assessed. Rolls-Royce applies $5 \times 5$ risk matrices combining measures of likelihood and impact to assess risk. The unit cost risk matrix relevant to the product being analysed is shown in Fig. 1 .

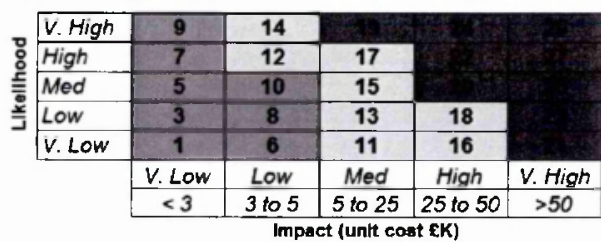

Fig. 1: Risk criteria case study 1

\section{Likelihood}

Assessing risk using the matrix in Fig. 1 requires the likelihood assessment method from the EC report to bc translated into the scoring scheme applied, using a scale of $v$. low to v. high. Based on the actual SR scores given in Table 2, Table 3 outlines how the SR translates into an assessment of likelihood consistent with the risk matrix.

Table 3: Translating SR scores into the risk matrix scale

\begin{tabular}{|c|c|}
\hline Like khood (L) & SR score \\
\hline Very high & SR $<4$ \\
\hline High & $3 \times S R<4$ \\
\hline Medium & $2 \times S R \leq 3$ \\
\hline Low & $1 \leq S R<2$ \\
\hline Very low & SR $<1$ \\
\hline
\end{tabular}

PGMs having a SR score of 3.6 translates into a high likelihood from Table 3 . 
Impact

To assess the impact, a breakdown of unit cost is required to understand how much of the total cost of turbine blades the materials account for. Illustrative cost data is presented in Table 4.

Table 4: Illustrative unit cost breakdown

\begin{tabular}{|l|r|}
\hline Element & Cost (£s) \\
\hline Casting & 600 \\
\hline Material & 60 \\
\hline Coating & 250 \\
\hline Machining & 150 \\
\hline & 1060 \\
\hline
\end{tabular}

An estimate of the potential cost increase of the material cost fraction is required, which is a function of:

1. How much the cost of the PGM could increase.

2. The PGM fraction of the whole material cost.

It is impossible to predict what the future cost of a material might be with any degree of certainty. To give an indication of the potential cost increase, the price volatility of the material is used. From company data the historical price volatility of the PGM in question is approximately 20 (calculated by dividing the maximum market price by the minimum taken over a 10 year period). For illustration, the PGM fraction of the cost of the material is estimated to be $1 / 3$. Thus the impact can be calculated by multiplying $1 / 3$ of the material price by 20 , shown in Table 5 .

Table 5: Impact on unit cost

\begin{tabular}{|l|r|r|l|}
\hline Ele ment & Cost (Es) & New cost (Es) & \\
\hline Casting & 600 & 600 & \\
\hline Material & 60 & $\mathbf{4 6 0}$ & \\
\hline Coating & 250 & 250 & \\
\hline Machining & 150 & 150 & \\
\hline & $\mathbf{1 0 6 0}$ & $\mathbf{1 4 6 0}$ & \\
\hline & & & Engine unit cost change \\
\hline & Change & 400 & $\mathbf{4 5 6 0 0}$ \\
\hline
\end{tabular}

As over 100 turbine blades are used in the engine, the total impact on unit cost of approximately $£ 45000$, which represents a high impact on the scalc shown in Fig. 1.

Risk assessment

Combining the high likelihood from Table 3 with the impact calculated above produces the risk assessment result in Fig. 2.

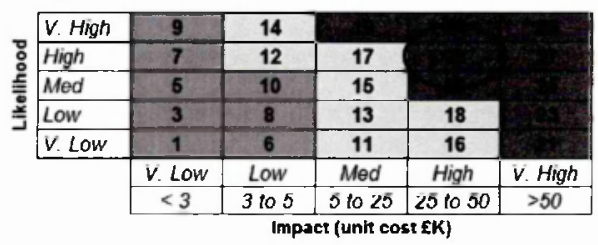

Fig. 2: Risk assessment result

Being the top right of the risk matrix this risk is deemed unacceptable and will require mitigating actions.

\section{Mitigating actions}

One mitigating action would be to take the PGM out of the blade. However, this will have an impact on specific fuel consumption (sfe), which is another design objective.
Risk criteria for assessing risks against $\mathrm{sfc}$ is shown in Fig. 3.

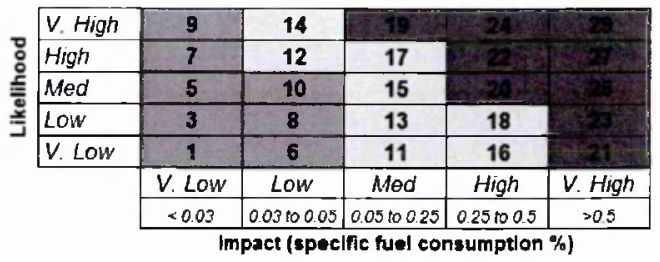

Fig, 3: Risk critcria for sfe

The impact on sfe of taking the PGM out of the blade is estimated to be between $0.1-0.2 \%$, from internal engineering data. An sfc reduction of this magnitude translates into a medium impact using the $\mathrm{x}$-axis scale in Fig. 3.

As the impact on sfe will only occur if an alloy not containing the PGM is used, which is dependent on the likelihood of the PGM beconing unavailable, the likelihood result from the unit cost assessment is used. Combining this with the sfc impact produces the risk assessment result shown in Fig. 4.

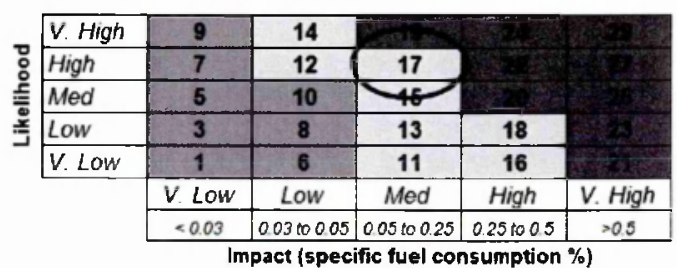

Fig. 4: Risk assessment result for sfc

$\Lambda s$ the risk to unit cost is higher than for sfe, on balance it is likely that the PGM would be removed and a different alloy used.

\section{CASE STUDY 2: ASSESSING RISKS TO NEW DESIGNS}

The following steps summarise the process for assessing the risks posed by the use of critical matcrials to new designs:

1. Select a BoM for a new design.

2. Identify critical materials used within the a BoM for a new design, by comparing it with the EC 14.

3. Complete product based risk assessment, using risk criteria relevant to the product in question, by multiplying the likelihood (supply risk) by the impact on business objectives.

A BoM for the outer liner of an annular combustor used on a small military turbofan engine is used for this case study, which is being re-designed to meet new customer requirements. Two uses of at risk elements were found in the new design:

1. Cobalt within the combustor alloy.

2. Yttrium (a REE), used within the thermal barrier coating applied to the combustor. 
As with the previous example, the case study will focus on the impact on unit cost objectives. The risk criteria taken from the relevant business risk management plan is shown in Fig. 5.

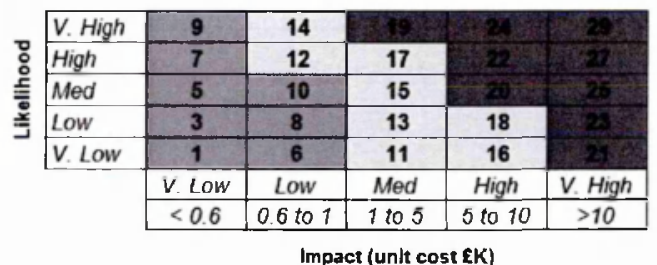

Fig. 5: Risk assessment criteria

\section{Likelihood}

From Table 2, the SR scores for cobalt and yttrium are 1.1 and 4.9 respectively. These scores translate into a low likelihood for cobalt and very high likelihood for yttrium using Table 3.

Impact

The unit cost breakdown for the component is required to assess the impact on unit cost objectives. Illustrative cost data is given in Table 6 .

Table 6: Illustrative unit cost brcakdown

\begin{tabular}{|l|r|}
\hline \multicolumn{1}{|c|}{ Element } & Cost [Es) \\
\hline Casting ops & 2500.00 \\
\hline Material & 500.00 \\
\hline Machining & 1250.00 \\
\hline Coating & 250.00 \\
\hline Rernaining ops & 1500.00 \\
\hline & $\mathbf{6 0 0 0 . 0 0}$ \\
\hline
\end{tabular}

As with the previous case, to understand the impact on unit cost, an estimate of the potential cost increase of the material cost fraction is required, which is a function of:

1. How much the cost of the cobalt and yttrium could increase.

2. The cobalt and yttrium fraction of the overall material and coating costs.

From company data, the historical price volatility of cobalt is 6 (max. price/min. pricc over 10 years) and for yttrium is 7. For illustration, cobalt is estimated to represent $20 \%$ of the total material cost and yttrium $3 \%$ of the coating cost. The impact is calculated by multiplying $20 \%$ of the materials cost by 6 (volatility of cobalt) and $3 \%$ of the coating cost by 7 (volatility for yttrium). The overall impact on cost is shown in Table 7.

Table 7: Unit cost impact

\begin{tabular}{|l|r|r|l|}
\hline \multicolumn{1}{|c|}{ Element } & Cost (fs) & New cost (fs) & \\
\hline Casting ops & 2500.00 & 2500.00 & \\
\hline Material & 500.00 & 1100.00 & \\
\hline Machining & 1250.00 & 1250.00 & \\
\hline Coating & 250.00 & 302.50 & \\
\hline Remaining ops & 1500.00 & 1500.00 & \\
\hline & 6000.00 & 6652.50 & \\
\hline & & & Engine unit cost change \\
\hline & Change & 652.50 & \\
\hline
\end{tabular}

The impact on unit cost of $£ 600$ for cobalt represents a low impact on the scale shown in Fig. 5. Impact on unit cost of $£ 52.50$ for yttrium is very low using the same scalc.

\section{Risk assessment}

Combining the low likelihood and impact for cobalt produces a low risk (Fig. 6). Combining the very high likelihood with a very low impact for yttrium also produces a low risk (Fig. 6). Aggregating the scores of impact and likelihood for both cobalt and yttrium gives a total materials criticality risk towards the centrc of the risk matrix in Fig. 6. A risk of this magnitude is unlikely to be of conccrn, not requiring mitigating actions. Being towards the centre of the risk matrix it may require monitoring to ensure the risk does not become unacceptable.

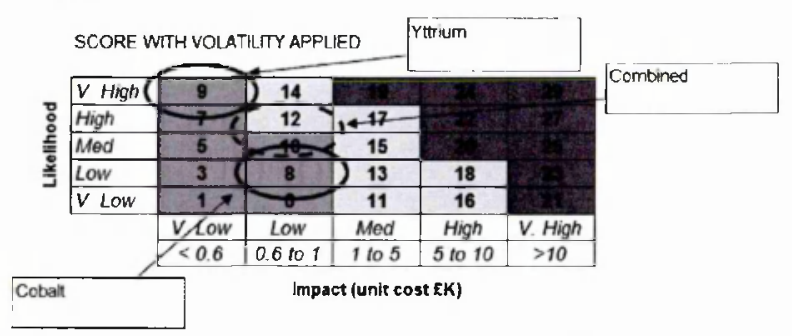

Fig. 6: Risk assessment result

\section{DISCUSSION}

The two case studies show how the risk based approach can be used to consider a complex environmental issue in a format that is consistent with standard engineering design decisions at Rolls-Royce. However, the approach is challenging for a number of reasons, discussed below.

There are significant uncertainties within all parts of the assessment. Firstly, assessing supply risk is not a simple task. The EC method was applied as it provided a transparent methodology and developing a bespoke approach was outside of the scope of this work. However, in reality there are many metrics that can be included within the assessment which were overlooked, for example risks related to materials produced as co-products or future changes in material demand. Adding both of these might have identified some of the 14 materials as being not critical, or highlighted at risk materials which have been overlooked. The method could be improved by selecting metrics that are relevant to the context (RollsRoyce), or even better using a dynamic modeling approach instead of relying on proxy metrics as representations of dynamic material supply systems [9].

Another area of uncertainty is in the product cost data used within the assessment. In a company with an extended supply chain, quite often a lot of the costs related to raw material inputs are not readily available. For the purposes of this paper illustrative costs were used. Although in practice cost data is difficult to get hold of. Costs can be estimated by comparing raw cost data from the purchasing function with cost breakdowns for parts. Engineering judgement can then be used to estimate what fraction of the cost of, for example a forging on a BoM, 
might be related to raw material inputs. Greater accuracy, and thus confidence in the results, could be obtained if the BoM (or other system) clearly outlined what costs elements were related to raw materials.

The most obvious area of uncertainty is predicting how a risk to supply may translate into an impact on cost. It is impossible to try and predict material prices. To obtain defendable estimates historical price volatility was used, although this must go with the caveat that relying on historical trends is a very poor means of estimating what might happen in the future [10]. For example, the price volatility for cobalt and yttrium were quite similar. Although the current price trend for cobalt is stable, while the shape of the yttrium curve is exponential. If this trend continued using past data is a very poor estimatc of the future impact. This problem is not likely to be resolved; there are always going to be uncertainties in predicting the future.

A final source of uncertainty is in the business objectives themselves. Risk can only be assessed against objectives. If the busincss alters the critcria used for the risk assessment (the scales of impact, likelihood and acceptability of risks) the risks presented here could be more or less acceptable. Assessing risk is inherently subjective.

Acknowledging these uncertainties, the paper has shown how the risk based approach is useful. One classic problem in ecodesign is determining how to trade-off between environmental impacts. Using the framework of business objectives, the first case study successfully demonstrated how this can be achieved.

\section{EXPANDING THE RISK BASED APPROACH}

A final consideration is how the risk approach could be expanded to include other environmental business hazards. Building from the two perspectives described in Section 3.2 , this could be achieved by defining more features of environmental hazards (for example the use of hazardous substances). This would also require more detailed product data containing features of products that could be connected to these hazards, with a life cycle view (for example, a life cycle inventory). Ways of measuring the likelihood of different hazards will also be required.

\section{CONCLUSIONS}

This paper has presented an approach to ecodesign based on environmental risk management, in conclusion:

- The risk bascd approach successfully demonstrates how a complex environmental problem could be considered with standard engineering decisions. It has also shown that by using business objectives, environmental impacts can be traded off with cach other.
- However, whilst the method was successful, there were significant uncertaintics. In particular assessing supply risk and how this translates into an impact on material price.

- The risk based approach could be expanded to include other environmental business hazards. This requires more detailed product data.

\section{REFERENCES}

[1] BSI (2009): Risk management - principles and guidelines, British Standards Institution (BSI), London.

[2] Matten, D. (1995): Strategy follows structure: environmental risk management in commercial enterprises, Business Strategy and the Environment, 4, pp. 107-116.

[3] Pennington, D.W., Potting, J., Finnveden, G., Lindeijer, E., Jolliet, O., Rydberg, T., Rebitzer, G. (2004): Life cycle assessment part 2: Current impact assessment practice, Environment International, 30, pp. 721-739.

[4] Graedel, T.E. (2007): Determining the criticality of materials, UNEP International Workshop on resource efficiency and recycling, Tokyo, September, United Nations Environment Programme (UNEP).

[5] Morley, N., Eatherley, D. (2008): Material secturity: Ensuring resource availability for the UK economy, C-Tech Innovation, Chester, UK.

[6] Duclos, S., Otto, J.P., Konitzer, D.G. (2010): Design in an era of constrained resources. Mechanical Engineering, American Society of Mechanical Engineers (ASME), September.

[7] European Commission (2010): Critical raw materials for the EU, European Commission, Brussels.

[8] Koizumi, Y., Kobayashi, T., Jianxin, Z., Yokokawa, T., Harada, H., Aoki, Y. and Arai, M. (2003): Development of next generation Ni-base single crystal superalloy. Proceedings of the International Gas Turbine Congress, Tokyo, 2-7 November, Gas Turbine Society of Japan.

[9] Alonso, E. (2010): Materials scarcity from the perspective of manufacturing firms: risks, effects and responses, $\mathrm{PhD}$ Thesis, Massachusetts Institute of Technology, Cambridge, MA, USA.

[10] Taleb, N., Goldstein, D.G., Spitznagel, M.W. (2009): The six mistakes exccutives make in risk management, Harvard Business Review, October, pp. 78-81. 


\title{
THE LIKELIHOOD OF RESTRICTIONS IN THE AVAILABILITY OF METALLIC RESOURCES
}

\author{
Stafford Lloyd ${ }^{(1,2)}$, Andrew Clifton $^{(1)}$, Lucia Elghali ${ }^{(2)}$, Chris France ${ }^{(2)}$ and Jacquetta \\ Lee $e^{(2)}$ \\ 1. Rolls-Royce, Gipsy Patch Lane, Filton, Bristol, BS34 7QE. \\ 2. Centre for Environmental Strategy, University of Surrey, Guildford, GU2 7XH.
}

\begin{abstract}
Some modern industries are becoming more reliant on exotic metallic resources, for example rare earth elements. There is increasing interest in managing the associated risks through assessing the vulnerability of material supply chains to supply restrictions. It is impossible to predict when or if a restriction might occur and what the exact impact might be either on availability or price. However, if a business is reliant on these resources, it is prudent to identify areas in the supply chain that might be at risk, as mitigating strategies can be put in place and taken if necessary. This paper examines the problem of defining a method that can be used to assess the likelihood of material supply restrictions. This includes (1) identifying metrics that have been used to assess the phenomenon, (2) defining which metrics should be selected for a given context, and (3) deciding how they should be aggregated, if at all, to give an assessment of the likelihood of a material supply restriction. A likelihood assessment method for use within RollsRoyce is then presented, which is part of a framework for environmental risk management currently being developed. The purpose of the framework is to manage the business risks posed by environmental problems more broadly.
\end{abstract}

Keywords: Business risks, environmental risks, resource scarcity, metalllc resources 
Proceedings of the 2011 Conference for the Engineering Doctorate in Environmental Technology

\subsection{Introduction}

The economic risks posed by restrictions in the availability of natural resources have been discussed within different disciplines for some time. Often termed 'materials scarcity', the phenomenon is concerned with constraints being placed on the accessibility of material commodities as a result of geological, environmental, social and economic factors. A historical example is the Cobalt crisis in the 1970s (Alonso et al. 2007).

The increasing reliance of modern industry on materials that are considered to be relatively scarce has recently raised awareness of the risks. There has been significant interest in analysing the problem from a macro-economic perspective (see DEFRA 2010, European Commission 2010, Morley and Eatherley 2008), although less examination of how the problem might be addressed at a business level. It is impossible for a business to predict when or if a supply restriction might occur, or what the impact might be on availability or price. However, if a business is reliant on the supply of scarce resources, it would be prudent to understand the risk to determine whether mitigating actions are required.

The risk posed to businesses by restrictions in the availability of materials is a function of: (1) the likelihood that the supply of a material will become restricted, either through increases in price or becoming physically unavailable; and (2) the impact this restriction will have on business objectives. A material is referred to as 'critical' when it is high risk i.e. it is likely to become unavailable and the consequences would be severe if this were the case.

This paper focuses on the problem of how to assess the likelihood of a materials supply restriction, to understand the business risks posed by materials scarcity. The problem is structured by (1) Identifying metrics that can be used to identify materials at risk of a supply restriction (2) determining which metrics should be selected, and (3) combining the selected metrics in order to identify materials that have a high likelihood of a supply restriction.

The problem is structured using a value function approach, which is typically applied to multiple criteria decision problems (Belton and Stewart 2002). The paper concludes with some comments on the limitations of using an assessment method based on the use of static indices. 


\subsection{Methodology}

Assessing the likelihood of a materials supply restriction exhibits the characteristics of a MCDA (Multiple Criteria Decisions Analysis) problem. The objective is to determine which materials are likely to be subject to a supply restriction, as an input to a broader risk assessment process. The alternatives are the materials themselves. To evaluate how the alternatives perform in meeting the objective different criteria are necessary, which are the metrics, used to assess whether a materials is likely to be restricted.

The first problem is to determine which criteria are to be used in the evaluation of the alternatives. Secondly a means of evaluating how each of the materials performs against the criteria individually is required. Thirdly, it is necessary to determine how the scores for each criteria should be aggregated, to provide a higher-level evaluation of the likelihood of a material supply restriction. The final requirement is related to how the likelihood assessment is used, which will be as an input to a business risk assessment process, requiring a numerical assessment.

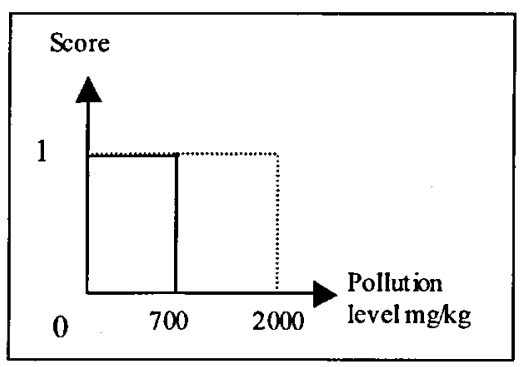

Figure 1: Example value function for pollution monitoring (Beinat 1997, p.9)

Of the various categories of multiple criteria modelling, value measurement models are an appropriate method for this problem (Belton and Stewart 2002, p.9). Value function methods are particularly suitable (see Beinat 1997), identifying upper and lower performance limits, for a given criteria, assigning them a score of one and zero. A mathematical representation of how performance is measured between the upper and lower limits is developed, which can be used to evaluate alternatives against individual criteria (Beinat 1997, p.9). A good example is in 
monitoring pollution levels, where a value function can be used to apply thresholds to evaluate pollution performance, as shown in Figure 1.

To further refine the problem as outlined, the following methodology will be applied to perform an assessment of the likelihood of a materials supply restriction (1) define metrics (our criteria) to be used for the assessment (2) develop a value function for the individual metrics (3) determine a higher level aggregation model to produce a result consistent with a simple likelihood scale applied within a business risk assessment matrix, and (4) produce example assessments for selected alternatives (materials).

\subsection{Metrics for assessing materials scarcity}

Historical examples of materials scarcity provide a set of factors that have led to supply restrictions in the past. Metrics can be developed from these factors to assess the likelihood of material supply restrictions in the future. There have been several reports published on the topic of materials scarcity in recent years. Sample metrics, taken from these sources, are outlined in Table 1.

Not all of the sources cited applied all of these metrics for their assessment, so the first problem is to decided which of those metrics listed in Table 1 are to be used. Deciding which metrics to be used inevitably requires judgements on the relevance and importance of the metrics available, which will depend on the objectives of the assessment, the context within which it is performed and the preferences of stakeholders in the assessment. There are also practical issues regarding the need for transparency and available data. 
Proceedings of the 2011 Conference for the Engineering Doctorate in Environmental Technology

Table 1: Metrics used to assess materials scarcity (Alonso et al. 2007, DEFRA 2010, Duclos et al. 2010, European Commission 2010, Morley and Eatherley 2008)

\begin{tabular}{|c|c|c|}
\hline Metric & Unit & Description \\
\hline Depletion indices & years & $\begin{array}{l}\text { Ratio of known reserves to current usage rates, providing the number of years of } \\
\text { supply remaining assuming no significant new discoveries. Can be static, assuming } \\
\text { no change in consumption, or dynamic, applying an increasing consumption rate. } \\
\text { Assumption: High depletion index increases likelihood. }\end{array}$ \\
\hline Geological abundance & $\underset{\%}{\mathrm{ppm} /}$ & $\begin{array}{l}\text { Can be measured as a mass fraction of earth's crust in part per million (ppm) or } \\
\text { based on ore concentration (\%). } \\
\text { Assumption: Lower abundance increases likelihood. }\end{array}$ \\
\hline Market price / volatility & $\$$ & $\begin{array}{l}\text { Price of commodity and / or its volatility, measured by dividing max. price by min. } \\
\text { price over time. } \\
\text { Assumption: High price / volatility increases likelihood. }\end{array}$ \\
\hline $\begin{array}{l}\text { Geographic / institutional } \\
\text { structure }\end{array}$ & $\%$ & $\begin{array}{l}\text { Diversity of supply base. Measured either geographically (monopoly) or } \\
\text { institutionally (oligopoly), on a supply or reserves (share of basis. } \\
\text { Assumption: Diversity decreases likelihood. }\end{array}$ \\
\hline Political stability & - & $\begin{array}{l}\text { Related to producing countries. World Bank or Failed State ind ices can be applied. } \\
\text { Assumption: Instability increases likelihood. }\end{array}$ \\
\hline Demand changes & - & $\begin{array}{l}\text { Likelihood of techno bogical de velopments increasing demand, reduxing availability. } \\
\text { Assumption: Demand increases likelihood. }\end{array}$ \\
\hline Recycling rate & $\%$ & $\begin{array}{l}\text { Ratio of scrap to total usage. } \\
\text { Assumption: Reliance on recycled content decreases likelihood. }\end{array}$ \\
\hline $\begin{array}{l}\text { Environmental country } \\
\text { risk }\end{array}$ & - & $\begin{array}{l}\text { Distance to target measurement of country performance against stated environmental } \\
\text { policy goals, developed by Yale University. } \\
\text { Assumption: Poor performance may restrict mining activities, increasing likelihood. }\end{array}$ \\
\hline $\begin{array}{l}\text { Environmental impacts of } \\
\text { production }\end{array}$ & various & $\begin{array}{l}\text { Measured per kg of material produced. Global warming potential measured in } \mathrm{CO}_{2} \\
\text { equivalents or total material requirement can be used for a simplified analysis. } \\
\text { Assumption: High environmental impact increases likelihood. }\end{array}$ \\
\hline Co-product dependence & - & $\begin{array}{l}\text { Materials that are produced as a by-product on another material's extraction process. } \\
\text { Assumption: Primary ore dependence increases likelihood. }\end{array}$ \\
\hline Substitutability & - & $\begin{array}{l}\text { How easily a material can be replaced with another one in any application } \\
\text { Assumption: Substitutability decreases likelihood. }\end{array}$ \\
\hline
\end{tabular}

The objective of this assessment is to assess the likelihood of a supply restriction from a business perspective, in particular from the perspective of Rolls-Royce and ideally the wider aerospace industry. It is necessary to elucidate the values of this industry in terms of how they perceive the problem of materials scarcity and which metrics would give the best indication of scarcity likelihood from this perspective. Workshops held within the industry have clearly highlighted three main requirements for an assessment method:

1. To build upon an established methodology, rather than 're-inventing the wheel'; 
2. To Use a method that is simple and transparent, with an easily repeatable calculation methodology;

3. To prioritise economic measures of scarcity, acknowledging the role of prices as being a proxy measure of scarcity in the short term.

Based on these requirements an assessment method built around that developed for the European Commission (see European Commission 2010) was created, which applies the following metrics: diversity of supply base, political stability, environmental country risk, recycling rate and substitutability. Additional metrics were considered necessary to provide a fully rounded picture, including a geological perspective and also a financial one, reflecting a preference for economic measures. The following metrics were added to those taken from the European Commission report: static depletion index and price volatility. More information on the European Commission method and selected metrics is provided below.

\subsection{European commlssion method}

The European Commission method (see European Commission 2010) merged the five selected metrics to produce two measures indicating the likelihood of a supply restriction: Supply risk (SR) and Environmental Country Risk (EM).

\subsubsection{Supply risk (SR)}

Supply risk is calculated by combining measures of supply diversity, political stability, recycling rate and substitutability, to produce a risk index for a material.

A measure called the Herfindahl-Hirshman Index $(\mathrm{HHI})$, which is often used in competition law, was used to provide a numerical valuation of supply diversity. The $\mathrm{HHI}$ can be calculated by summing the squares of the supply percentages $\left(S_{i c}\right)$ of producing countries $(c)$, for a given material:

$$
H H I_{c}=\sum_{c} S_{i c}^{2}
$$


The result of the $\mathrm{HHI}$ should produce a score of between $\mathbf{0}$ and 10000 , with a higher score reflecting lower supply diversity and a greater likelihood of a supply restriction.

The EC report merged a measure of supply diversity with a measure of political stability, to highlight where the supply of a material was dominated by producing countries that are politically unstable. The combination of monopoly conditions with an unstable producing region will increase the likelihood of a supply restriction. For political stability, the EC report used World Governance Indicators (see World Bank 2010). The indicators use 6 categories to produce a World Governance Indicator (WGI) for each country, on a scale of 0 to $10 ; 10$ representing the most unstable countries and 0 the most stable. WGI scores were used to produce a modified $\mathrm{HHI}$ as shown in equation 2:

$$
H H I_{W G I}=\sum_{c} S_{i c}^{2} W G I_{c}
$$

The WGI modified $\mathrm{HHI}$ produces a score of between 0 and 100000 , which can be divided by 10000 to produce a result on a scale of 0 to 10 .

The recycling rate used for a material $i\left(\rho_{i}\right)$ applied the ratio of current demand met by old scrap i.e. end of life products. The assumption is that reliance on recycled content reduces risk. Data on recycling rate is given in the European Commission report.

Substitutability $\left(\sigma_{i}\right)$ for a material was measured using an index developed through expert judgements, with the indices calculated in the European Commission report using the scale outlined in Table 2.

Table 2: Substitutability indices (European Commisson 2010)

\begin{tabular}{|l|l|}
\hline Score & Substitutability \\
\hline 0 & Easily and completely substitutable at no additional cost. \\
\hline 0.3 & Substitutable at low cost. \\
\hline 0.7 & Substitutable at high cost and/or loss of performance/ \\
\hline 1.0 & Not substitutable. \\
\hline
\end{tabular}

The supply risk is calculated by multiplying the $\mathrm{HH} \mathrm{WGI}_{\mathrm{WG}}$ scores by the recycling rate $\left(\rho_{i}\right)$ and substitutability index $\left(\sigma_{i}\right)$ as shown below: 
Proceedings of the 2011 Conference for the Engineering Doctorate in Environmental Technology

$$
S R_{i}=\sigma_{i}\left(1-\rho_{i}\right) H H I_{W G I}
$$

(Equation 3)

The term $\left(1-\rho_{i}\right)$ is used as a higher recycling rate will reduce supply risk. The result of the supply risk caiculation will produce a score of between 0 and 10 by material, with a score of 10 representing the highest possible risk.

\subsubsection{Environmental Country Risk (EM)}

Using an Environmental Performance index (EPI) developed by Yale University (see Yale 2010), the environmental country risk is calcuiated in the same way as supply risk, except the $\mathrm{HHI}$ is modified by the EPI. The EPI analyses how close countries are to estabiished environment policy goals, producing an index on a scaie of 0 to 10 similar to the WGI; 10 signifying the worst performing countries. Mining activities can contribute significantly to environmental damage and the assumption is that the further a country is from stated policy goals, the more likeiy a restriction wil be placed on mining activities. The $\mathrm{HHI}_{\mathrm{EPI}}$ is calculated as shown below:

$$
H H I_{E P I}=\sum_{c} S_{i c}^{2} E P I_{c}
$$

The environmental country risk (EM) is calculated as shown below:

$$
E M_{i}=\sigma_{i}\left(1-\rho_{i}\right) H H I_{E P I}
$$

The EM again produces a score between 0 and 10,10 signifying the highest risk materials.

\subsection{Static depletion index (SDI)}

Static depletion index (SDI) is calculated by dividing the known reserves of a given material resource $i$ by current rates of consumption, providing the number of years of resource remaining at current consumption levels:

$$
S D I_{i}=\operatorname{Re} \text { serves } / \text { Consumption }
$$

(Equation 6) 
The measure assumes that consumption will remain constant, as will known reserves. The metric was applied by Alonso et al. (2007), Morley and Eatherley (2008) and DEFRA (2010), who all provided a view on SDI values that should give cause for concern, which is typically around 40 years.

\subsection{Price volatility (PV)}

Price volatility (PV) is calculated by dividing the maximum price of a material by the minimum price over a set time period:

$$
P V_{i}=\text { Max.price }_{\text {Min.price }}{ }_{10 \text { years }}
$$

Ten years is typicaily used. Price information is generally available through internet sources, for example the London Metal Exchange (LME), or is held as internal company data. DEFRA (2010) states that a price volatility of over $300 \%$ should create concern.

\subsection{Likelihood index (LI)}

The previous sections defined the metrics to be used for a likelihood assessment. The next issues were to (1) develop performance relationships for each individual criteria, which will be used to assess how likely a material is to become unavailable, and (2) develop a means of aggregating scores for the individual criteria into a higher-level likelihood assessment, in a way that is consistent with use in a risk management matrix.

\subsection{Individual criteria performance measures}

As discussed in the methodology, a value function method approach will be used for individual criteria performance scores. Value functions for the supply risk, environmental country risk, static depletion index and price volatility are presented in the following sections. 


\subsubsection{Supply Risk and Environmental Country Risk}

The European Commission report set the boundary of concern for supply risk at 1 (European Commission 2010), which provides quite a coarse assessment. To provide a finer assessment, a value function has been developed with boundaries at 1 and 3 and only materials with a SR score of over 3 will be considered high risk (Figure 2). This judgement reflects SR scores given in the European Commission (2010) report.

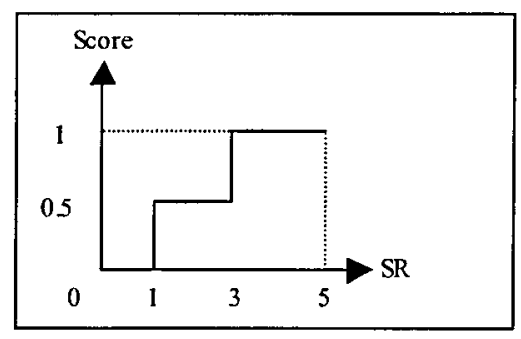

Figure 2: Supply risk value function

As the environmental country risk is taken from the same report a similar value function has been applied, which is shown in Figure 3.

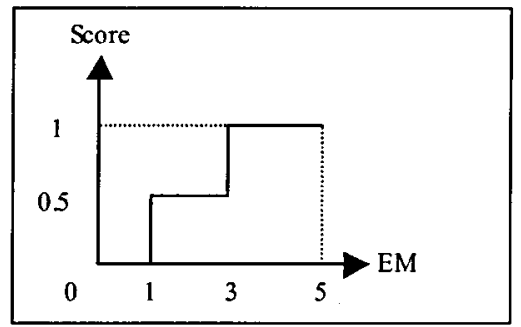

Figure 3: Environmental country risk value function

\subsubsection{Static Depletion Index}

DEFRA (2010) provides levels of concern for the SDI, stating that any materlal with a SDI under 40 years should be considered high risk and any material with a SDI of less than 80 years should give some cause for concern. A value function representing these judgements is shown in Figure 4. 
Proceedings of the 2011 Conference for the Engineering Doctorate in Environmental Technology

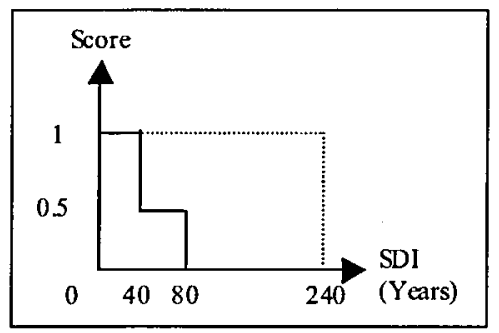

Figure 4: SDI value function

\subsubsection{Price Volatility}

Rolls-Royce holds price information on key commodities, which tracks price volatility over 10 years. By plotting the price data on a graph, bands categorising the magnitude of PV per material could be observed, which are represented in the value function shown in Figure 5.

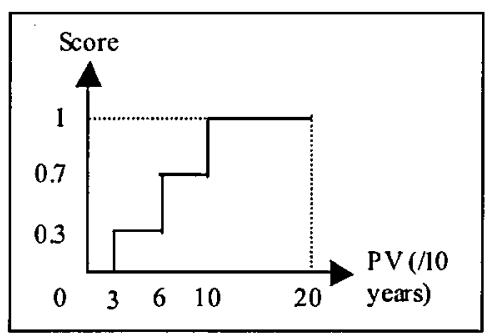

Figure 5: Price volatility value function

\subsection{Aggregation}

A combinatorial logic is required to aggregate each of the individual value function performances to provide a higher assessment of likelihood. The main requirements is for the final result to be consistent with a simple scale, similar to the one shown in Table 3 , that is consistent with the scoring scheme used within a standard risk assessment matrix. There are also the requirements of simplicity and transparency, coupled with a stated preference for economic measures of materials scarcity. 
Table 3: Likelihood scoring scheme for use in risk assessment

\begin{tabular}{|c|c|}
\hline Likelihood & Score \\
\hline High & $4-5$ \\
\hline Medium & $3-4$ \\
\hline Low & $1-3$ \\
\hline Very low & $0-1$ \\
\hline
\end{tabular}

A likelihood index for aggregating the individual value function scores is presented in Figure 6. The approach is a simple aggregation, where the performance of a material in each criteria is added together, providing a maximum possible score of 5 and minimum 0 . The score in the price volatility value function is double weighted, reflecting preferences for economic measures, giving a maximum score of 2 for this criteria. It is unlikely that a material will score highly in each category, however a material exhibiting a high score in individual categories is likely to present a higher likelihood of a supply restriction. To reflect this, alongside the simple combinatorial logic, further logic will be applied whereby if a material scores a maximum in any individual criteria, it will be considered to have the maximum (very high) assessment of likelihood in aggregation.

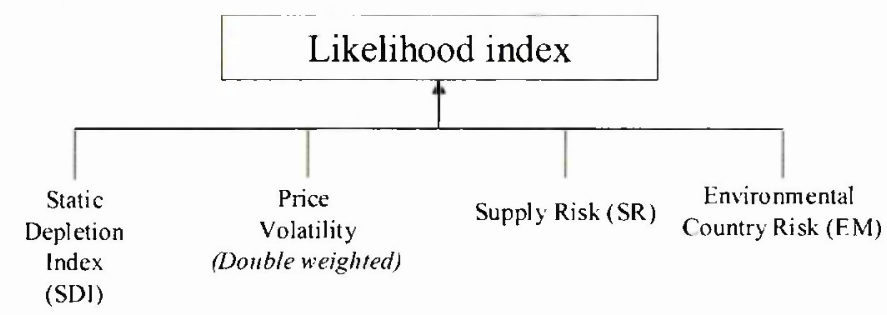

Figure 6: Likelihood index (LI)

\subsection{An example application of the method to some materials}

The following sections apply the assessment method to selected materials of recognised importance to the aerospace industry, as the final part of the assessment. 


\subsection{Rhenium (Re)}

The likelihood index for rhenium ( $\mathrm{Re})$ is presented in Figure 7. Rhenium is a main component of temperature resistant superalloys used to manufacture the turbine blades within a jet engine. Geologically it is relatively scarce and is produced in low volumes as a by-product. Although Rhenium has a LI score indicating low risk, both the SDI and PV scores are on the borderline of the highest score, which would make it very high risk. It would be sensible to monitor the performance of rhenium in the least, or even consider it as a higher risk material, for which mitigating actions might be required dependant on its use within the business.

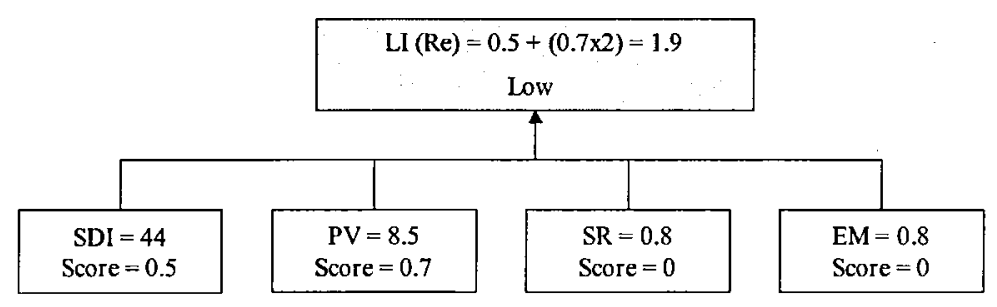

Figure 7: Rhenium result

\subsection{Copper (Cu)}

The likelihood index for copper $(\mathrm{Cu})$ is presented in Figure 8 . Copper is widely used in developed economies. The score indicates a very high risk due to copper's SDI score. The high risk of copper has recently been reflected in high and volatile prices (Alonso 2010). It would be sensible to consider how restrictions in the availability of copper would affect business objectives, to determine if actions are required to mitigate the risks.

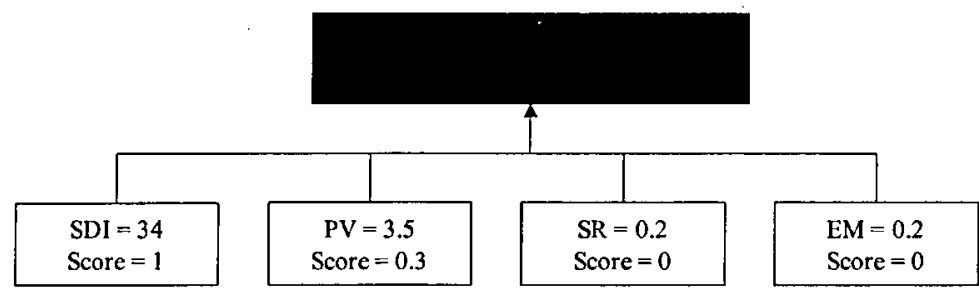

Figure 8: Copper result 


\subsection{Platinum (Pt)}

The $\mathrm{LI}$ score for platinum (Pt) is shown in Figure 9. Platinum (Pt) is known to be a potentially critical element, being expensive, geologically scarce and having few sources of supply. Dispersive uses, for example as a catalyst, also make it difficult to recycle, increasing dependence on primary production. The $\mathrm{LI}$ score indicates a very high risk, predominantly due to its supply concentration, reflected in the maximum score for Supply Risk. It would be prudent to understand business uses of platinum and perform a full business risk assessment to determine if any mitigating actions are required.

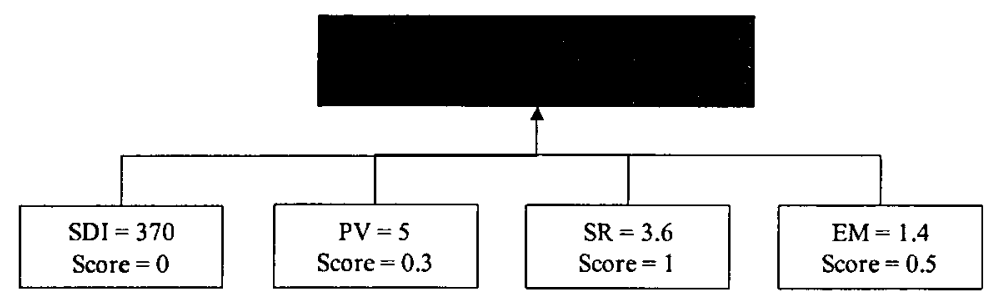

Figure 9: Platinum result

\subsection{Limitations of the static approach}

Alonso (2010) highlights some limitations of a static metric based approach and how reducing the complexity of the problems to simple figures obfuscates uncertainties in the data and how it might affect the results obtained. There is also the observation that static metrics "do not capture the dynamics of the material systems they seek to represent" (Alonso 2010, p. 61). Ideally a dynamic modelling approach would be developed, which is the subject of active research. A metric based assessment could be used to determine which materials require the modelling effort.

\subsection{Conclusions}

This paper has outlined the problem of assessing the likelihood of a materials supply restriction, in conclusion. The methodology applied a value function approach to develop numerical performance measures for materials against agreed assessment criteria. The criteria for assessing materials scarcity, the metrics, should be selected on the basis of the objectives of the assessment and the context within which it is performed. To get a representative position, a 
cross industry workshop was used to determine the criteria for an assessment method. Value functions and an aggregation model were developed on the basis of the criteria selected, and applied to provide likelihood indices for rhenium, copper and platinum. A dynamic modelling approach using future scenarios would provide a more robust assessment method, although a static metric approach is useful as a first pass at the problem.

\subsection{Acknowledgements}

Financial support from the Engineering and Physical Sciences Research Council and the Technology Strategy Board has contributed to this research.

\subsection{References}

Alonso, E., Gregory J., Field, F. and Kirchain, R. (2007) 'Material availability and the supply chain: Risks, effects and responses', Environmental Science and Technology, 41 (19), pp. 6649-6656.

Alonso E. (2010) Materials scarcity from the perspective of manufacturing firms: Effects and strategies. PhD Thesis, Massachusetts Institute of Technology.

Beinat, E. (1997) Value functions methods for environmental management and assessment. Dordrecht: Kluwer.

Belton, V. and Stewart, T.J. (2002) Multiple Criteria Decision Analysis: An integrated approach. Dordrecht: Kluwer.

DEFRA (2010) Review of the future resource risks faced by UK business and an assessment of future viability. London: Department for Environment, Food and Rural Affairs (DEFRA).

Duclos, S., Otto, J.P. and Konitzer, D.G. (2010) 'Design in an era of constrained resources', Mechanical Engineering, September, pp. 1-7.

European Commission (2010) Critical raw materials for the EU. Brussels: European Commision.

Morley, N. and Eatherley, D. (2008) Materials security: Ensuring resource availability for the UK economy. Chester: C-Tech innovation Ltd.

World Bank (2010) Worldwide governance indicators. Available at: http://info.worldbank.org/governance/wgi/index.asp. (Accessed 11 May 2011).

Yale (2010) Environmental Performance Index. Available at: www.epi.yale.edu. (Accessed: 11 May 2011) 